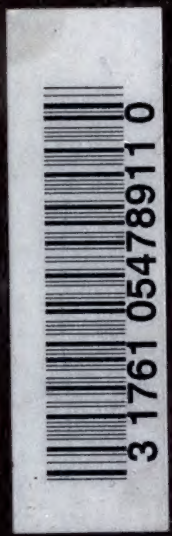

FIELD BOOK

OF

PRACTICAL MINERALOGY.

HOW TO EXAMINE

AND REPORT ON MINES.

MILLER.

S\&M

A

321 


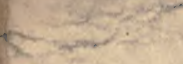
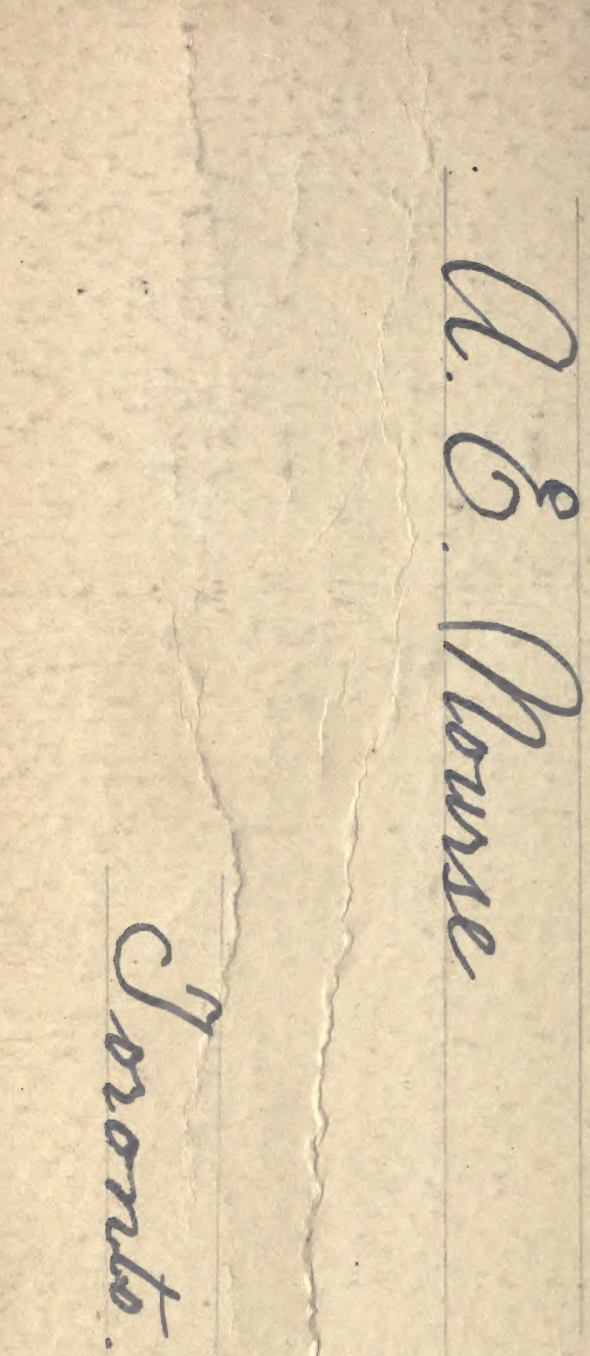
a.

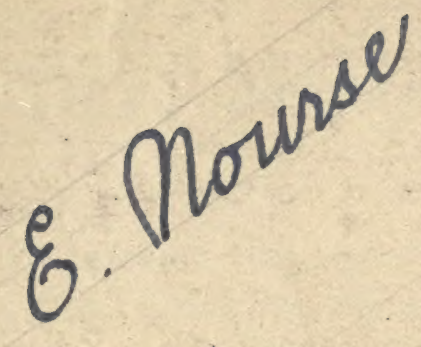

$y 0^{0^{10}}$ 



\title{
FIELD BOOK of
}

\section{Practical Mineralogy}

\author{
How to Examine \\ and Report on Mines
}

Designed for the Use of Prospectors, Mining Men, Engineers, and Others

B Y

G. W. MilleR, E. M., C. E.

SECOND EDITION

THE PUBLISHERS PRESS ROOM COMPANY, 1742-1748 STOUT STREET

DENVER, COLO.

1902 
COPYRTGHTED BY

The Publishers Press Room Company DENVEr, Colorado 1901

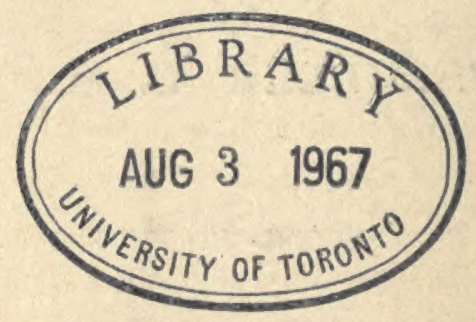




\section{Preface}

The publication of this little volume is the result of a long cherished plan; although not as complete in its discussion of the subject of ore deposits, the examination of mines, assaying, etc., as were the author's original intention, these omissions are thought to have been compensated for in part by giving more space to the subjects of determinative mineralogy and blow-pipe analysis. In the composition and compilation of the subject matter herein contained, all due precautions have been observed in order to render the information perfectly reliable and trustworthy, and to this end all descriptions of minerals appearing in the tables were carefully compared with those given in the following named standard works on the subject of mineralogy: System of Mineralogy, by E. S. Dana; Determinative Mineralogy and Blowpipe Analysis, by Brush \& Penfield. Other valuable authorities consulted are: Ore Deposits, by J. A. Phillips; Ore Deposits of the United States and Canada, by J. F. Kemp, and A Manual of Practical Assaying, by H. V. F. Furman. The names of all authorities consulted are mentioned in the foot notes.

Where inadvertently omissions have occurred and due credit has not been given, the author would esteem it a favor to have his attention called to the neglect. He would also be pleased to receive criticisms on the work so that he may be able to take advantage of them should a future edition be called for. It will be observed that in order to render the information of this little book 
more compendious and comprehensive for the benefit of the general reader, the usual plan of the more elaborated scientific text books could not be followed, and it would seem that with this and the aim of the little work fully understood, no other apology for its appearance in the present simple form is necessary. It will be further observed that (the design and object of) this little work is intended for the benefit of practical mining engineers, mineralogists, prospectors, mining men, etc., who feel the want of a ready reference field compendium of mining and mineralogical information, and who have not the time to grapple with theories or complicated formulæ. The work is divided into four parts :

Part I treats briefly of the subject of Ore Deposits. These are illustrated by nine wood cuts, showing the more typical classes of mineral veins, and other classes of ore deposits, and they are intended to aid the young miner in making the proper classification of all forms of ore deposits coming under his observation.

The several Theories, claiming to account for the filling of metalliferous veins are briefly given; then four graphic illustrations of Faults, with rules for finding the faulted portion of veins, are fully discussed; or at least it is believed that enough has been said to give the novice the correct idea of how to proceed in finding the vein should he meet with similar problems in practice.

Under the title "The Examination of Mines," after briefly defining the object of mining examination, the method of sampling and estimating the ore in sight is illustrated by drawings, and it is believed that this with other hints and instructions given on this subject will render the undertaking of making a mining examination quite easy, even in the hands of the young miner. 
"The Form of a Mining Report" next following is one universal in its application. It is a form extensively used by mining engineers, and may be modified so as to suit any particular case.

Part II gives the method and formulæ for assaying Gold, Silver, Lead and Copper ores. Methods for making Laboratory Tests of gold and silver ores, by the Cyanide, Chlorination and Amalgamation Processes are described. Then follows the subject of Blowpipe Analysis with a full descriptive list of blowpipe tests of the more common metallic substances and minerals met with.

Part III is devoted wholly to the subject of "Determinative Mineralogy." After discussing the physical properties of minerals and giving all necessary rules for their determination, the Analytical Tables of minerals are given.

The system of grouping minerals according to the most prominent metal or element entering into their composition has been adapted. Upwards of 345 of the more common and useful minerals are described, and in such a way as to admit of identification wherever met with in nature. The design of these tables is (in part) original with the author, and the plates from which they were made were prepared at a considerable cost by the skilled engraver, Mr. Selig Olcovich of Denver, Colo. One peculiar feature of these tables is that the plate containing the names of the minerals and their analytical description is made to fit the single page of the book and both sides of all pages are utilized. In the columns of each page is given, first, the names of the minerals; in the second column, their chemical composition (not in formulæ; but the names of all metals and elements are written out with the percentage of each ele- 
ment when known); in the third, lustre; fourth, color; fifth, hardness; sixth, streak; seventh, fracture and cleavage; eighth, tenacity; ninth, crystalline system; tenth, fusibility, and eleventh, specific gravity. It will be seen at once that with these properties known, the identification of any mineral described is made quite certain.

Part IV treats on the subject of "Naming Rocks." The more common rocks only are described and in such a way as in most cases to admit of at least approximate identification when met with. A Glossary of Mining Terms forms the next subject, then follows an arrangement of mineral collections, models, assay and blowpipe outfits. This concludes the work.

G. W. Mrluer, Butte, Mont., May 1st, 1901.

\section{PUBLISHERS' NOTE ON SECOND EDITION}

In placing before the public the second edition of Mr. Miller's work, we desire to state that the demand for the Field Book of Practical Mineralogy has far exceeded our expectations. Though we realized from the start that the work filled a recognized gap in mining literature, we were not prepared for the widespread interest with which it was greeted, not only in America but throughout the entire world where the mining industry thrives. One book sent to a locality has almost invariubly been followed by a shower of orders, so immediate is the appreciation of the value of Mr. Miller's labors among practical miners and prospectors everywhere. As a time saver, presenting in concrete and convenient form the facts of mineralogy from the miner's standpoint, this little volume appears to occupy a field peculiarly its own.

THE PUBLISHERS' PRESS ROOM CO.

Denver, Colorado, May 1, 1902. 


\section{INDEX TO SUBJECTS}

PART I.

Page.

Ore Deposits...................... 13.

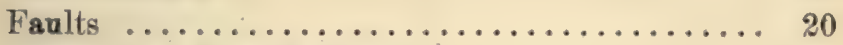

Theories of Vein Filling.............. 24

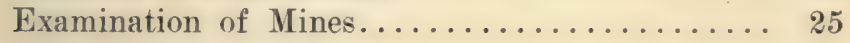

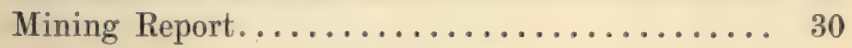

PART II.

Notes on Assaying................ 37

Gold and Silver Assaying. ............ 37

Lead Assaying................. 38

Copper Assaying. ................. 39

Laboratory Tests of Gold and Silver Ores...... 42

Cyanide Process...................... 43

Chlorination Process................ 45

Amalgamation Process............... 45

Blowpipe Analysis of Minerals........... 46

Blowpipe Operations.................. 48

Qualitative Tests..................... 54

\section{PART III.}

Determinative Mineralogy............. 85

Physical Properties of Minerals........... 86

Introduction to Analytical Tables of Minerals... 93

Analytical Tables of Minerals-Division I..... 95

Gold Telluride Minerals............... 96

Silver Minerals.................... 97

Copper Minerals.................... 100

Lead Minerals....................... 103

Zinc Minerals...................... 107

Cobalt and Nickel Minerals............. 108

Mercury Minerals.................... 110

Iron Minerals.................... 111 
Manganese Minerals................... 113

Cadmium, Tin and Titanum Minerals........ 114

Uranium and 'Tungsten Minerals........... 114

Cerium, Yttrium, Eithium, Lanthanum and

Didymium Minerals................ 116

Aluminium Minerals.................. 11\%

Magnesium Minerals................. 120

Calcium Minerals................... 120

Barium, Strontium, Potassium, Sodium and

Ammonium Minerals............... 122

Sulphur, Tellurium, Boron and Molybdenum.... 124

Arsenic, Antimony, Bismuth and Carbon....... 124

Hydrocarbon Minerals.................. 126

Mineral Coal...................... 126

Silica and the Silicates-Division II......... 128

Silicia or Quartz...................... 129

Bisilicates ........................... 131

Unisilicates ....................... 133

Scapolite Group......................... 135

Feldspar Group...................... 136

Mica Group............................ 137

Subsilicates ...................... 138

Hydrous Subsilicates-Chlorite Group........ 140

Hydrous Silicates-Zeolite Group........... 142

Hydrous Silicates................... 143

\section{PART IV.}

Naming Rocks.................... 149

Unstratified, Metamorphic and Eruptive Rocks.. 150 Stratified, Sedimentary or Aqueous Rocks....... 157 Glossary of Mining Terms.............. 162 Collection of Minerals, Models, Assay and

Blowpipe Outfits................. 182

Index to Minerals.................. 187 


\section{PART I.}

\section{Examination of EMines}





\section{PART I.}

\section{ORE DEPOSITS.}

CLASSIFICATION.

Ore Deposits, in all their various forms of occurrence in nature, may be briefly defined as being metalliferous aggregations occupying receptacles (made contemporaneous with, or previous to, the time of deposition) in the earth's crust.

The class to which the several forms belong will depend upon the mode of filling, the nature of the ore mass itself, the kind of cavity or receptacle containing the ore, and the position and relation of the ore deposit to the enclosing country rocks.

To sum up the several classes as they are exhibited in nature and revealed by extensive excavations in the many mining districts throughout the globe, the following classification seems proper:

I. Stratified Veins, or Beds.

II. Contact Veins.

III. True Fissure Veins.

IV. Segregation Veins or Deposits.

V. Massive or Chamber Deposits.

VI. Gash Veins.

VII. Inpregnation Deposits.

VIII. Stockwork Deposits.

IX. Fahlband Deposits.

The several sketches of the more typical varieties or classes of ore deposits as outlined above and exhibited on the following pages are intended to convey to the 
14. Ore Deposits.

young miner the proper conception of all forms met with, and to afford him a means of identifying them wherever observed in nature.

In making mining examinations it is important to bear in mind that on the nature and class of the ore deposits will depend in a great measure the probable continuity or permanency of the mine, and the method to be employed in mining the ore.

Fig. 1 represents a Stratified Vein, Bed or Deposit. These are metalliferous aggregations inclosed between sedimentary rocks, belonging to every geological age. They lie parallel to the stratification of the enclosing

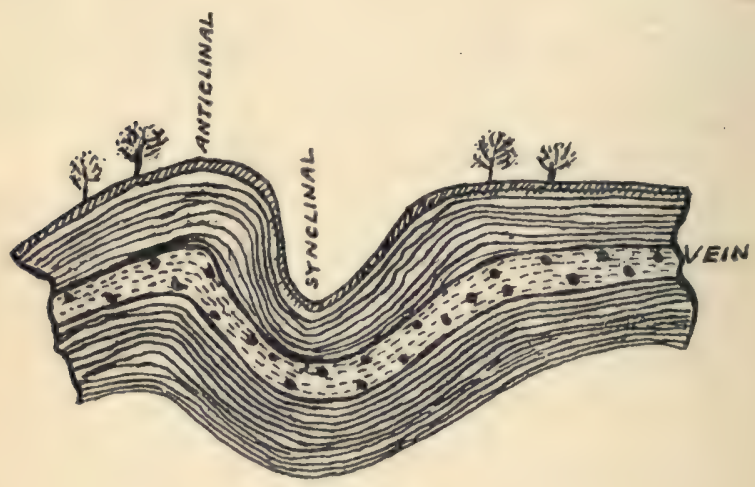

FIG. 1.

Stratified Vein or Bed.

rocks, and follow all their contortions. In this way ore beds form Synclinals or basins, and Anticlinals or saddles. When this class of deposit lies horizontal, or nearly so, they are called by miners, "Blanket Veins." 


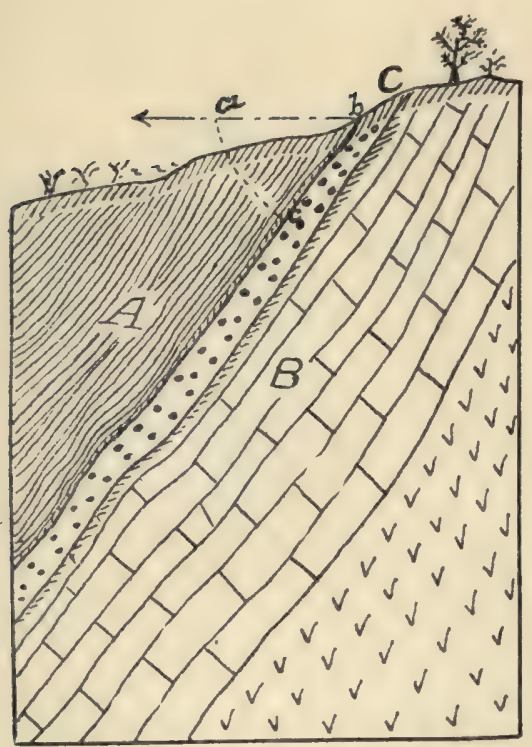

FIG. 2.

\section{Contact Vein.}

Fig. 2 represents a Contact Vein or Deposit. In this class of ore deposits the metalliferous accumulations or vein, is found between the planes of contact of dissimilar rocks, which are unlike in their mineralogical characteristics. Thus in the figure the portion A, is slate, and forms the Hanging Wall, while $\mathrm{B}$ is limestone, and forms the Foot Wall; $a, b, c$, measures the Dip Angle of the vein, while its Strike is the course ilong the plane of contact, and $\mathrm{C}$ is the Apex of the rein. 
16. Ore Deposits.

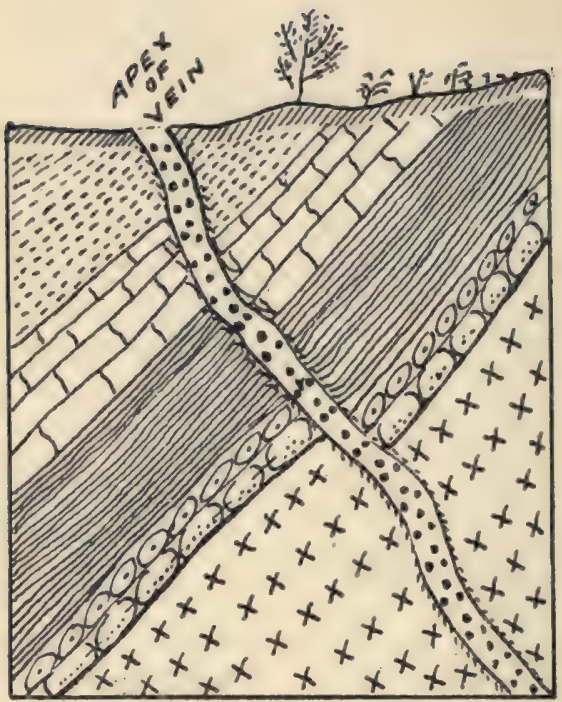

FIG. 3 .

True Fissure Vein.

Fig 3 represents a True Fissure Vein. This class of veins traverse the rocks independently of their structure, and are not parallel to the foliation or stratification of the enclosing rocks. Veins of this class are firther distinguished by the presence of mud seams along the walls; ore in banded structure, and by generally striking in a northerly direction.

They have originated in dislocations caused by extensive movement of the earth's crust and are therefore believed to extend indefinitely in depth. 
Fig. 4 represents that class of ore deposites known as Segregation Veins or Deposits. Th is class of ore deposits differ from the fissure vein type, in that their dip and strike conforms with the bedding planes of the enclosing rocks. The ore masses

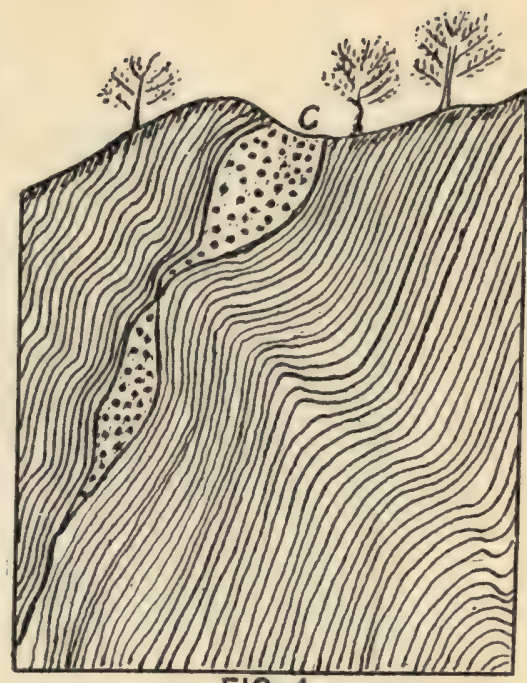

FIG. 4.

Segregation Vein or Deposit.

are more or less lenticular in shape. The ore may or may not outerop at the surface $C$.

Fig. 5 represents that

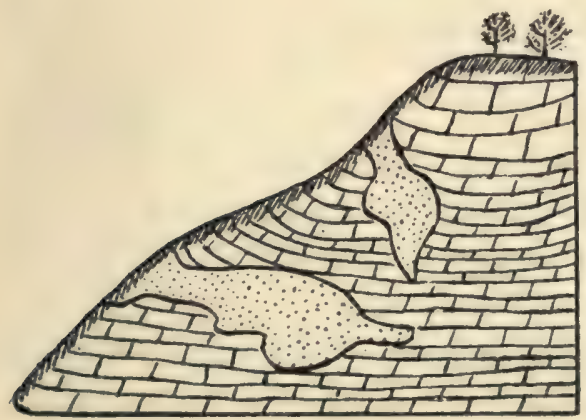

FIG. 5.

Massive or Chamber Deposit. class of ore deposits desig. nated as Massive or Chamber deposits. To this class of deposits belong such as occur in Bisbee, Ariz., Eureka, $\mathrm{Ne}$., etc. They are great chambers in limestone rocks form. ing receptacles for the ores of various metals, and are the source of much mineral wealth. 


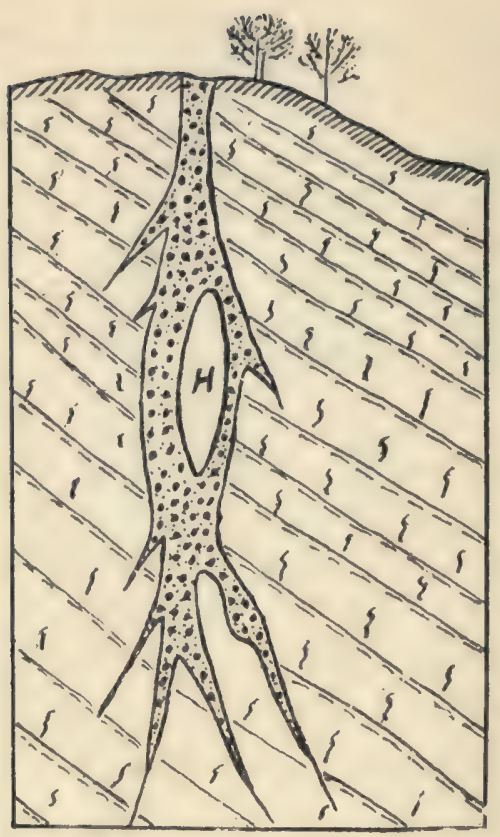

FIG. 6.

Gash Vein.

Fig. 6 illustrates a typical variety of that class of ore deposits known as Gash Veins. These are surce cracks filled with mineral matter, often taken for true fissure veins, from which they differ by their irregularity and want of continuity in strike, and dip. They thin out in sharp tapering points, divide and disarpear altogether at inconsiderable depths. A common occurrence in gash veins is what is called a horse $(\mathrm{H})$; this, together with the absence of selvage or gouge on the walls will generally distinguish them from true fissure veins. The gash vein class of veins are of common occurrence. and are met with in all mining countries. 
Fig. 7 exhibits

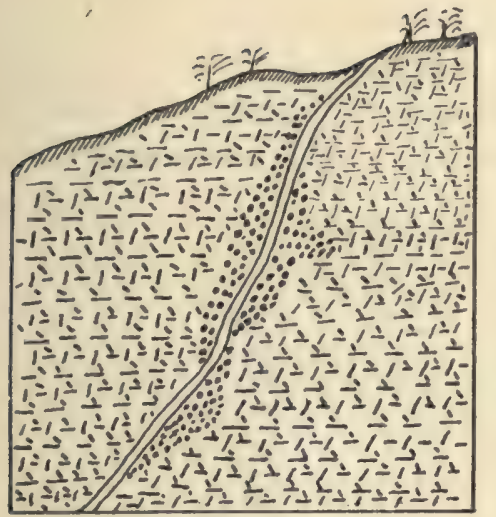

FIG. 7.

Impregnation Vein or Deposit.

that class of ore deposits known as Impregnation Deposits. In this class the metalliferous aggregations do not possess any regular outlines, and the ore is generally disseminated through the enclosing rocks forming irregular clusters on either side of the fissure.

Although met with in formations of almost every age they occur most frequently in igneous and other crystalline rocks.

Fig. 8 illustrates that class of ore deposits known as Stockwork Deposits. This form is closely allied to the impregnation class. They consist of a

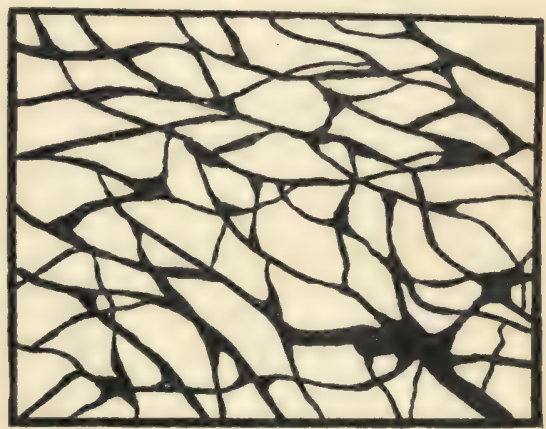

FIG 8.

Stockwork Deposit. network of small veins interlacing one another and traversing the rocks in various directions, the whole of the ore present is not, however, confined to the veins, a considerable portion of it being contained in the enclosing rock itself. 
Fig. $8 \mathrm{a}$ is a horizontal section representing that class of ore deposits known as Fahlbands. At Kongsberg, Norway, and vicinity, this class of deposits is worked for the silver ores contained in the Fahlbands; these are parallel belts of micaceous rocks of considerable width

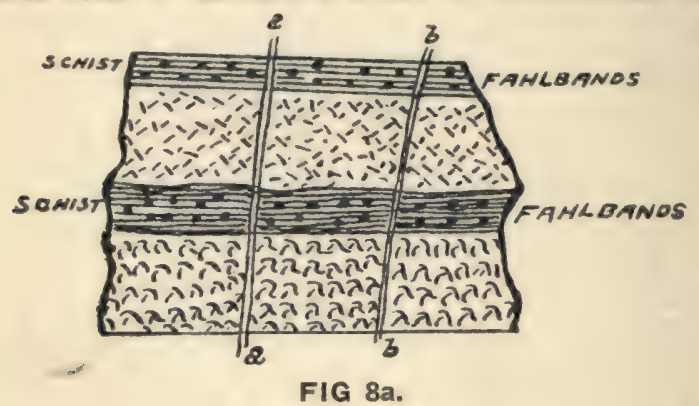

Fahlband Deposit.

and length. The narrow east and west fissure veins $a \quad a, b \quad b$, are highly productive when they intersect the Fahlbands and non-productive when they pass into the igneour or crystalline rocks on either side. No deposit of this class, to my knowledge, occurs in America.

\section{FAULTS.}

Fig. 9 exhibits a Normal Fault. The portion of the vein $\mathrm{D}$ D has moved downward along the fault plane $\mathrm{A}$ $\mathrm{B}$, from $\mathrm{C}$ to $\mathrm{D}$. The vertical dislocation is equal to the distance $a b$, and is called the throw of the fault. The

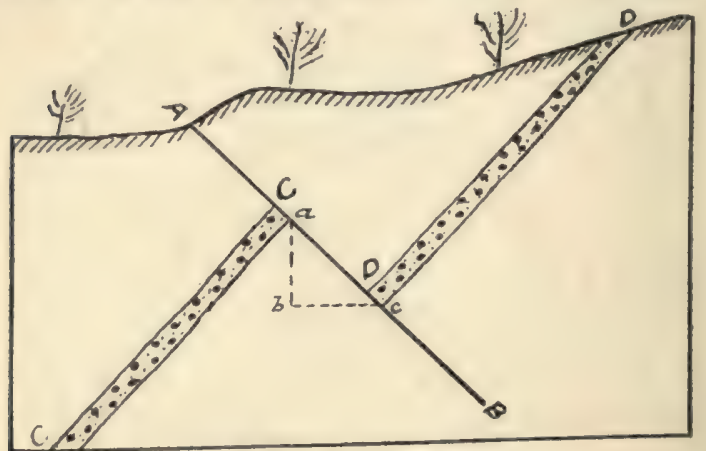

FIG. 9.-Normal Fault. 
horizontal dislocation $b \quad c$, is called the heave of the fault.

Law of Normal Faults: To find the continuation of the vein, in this case, we assume that the right hand side of the figure has moved downward along the fault plane $A B$, the motion thus conforming to the law of gravitation; if, therefore, the vein was lost at $\mathrm{D}$, we would naturally expect to find its continuation somewhere on the fault plane in the direction C. If lost at $\mathrm{C}$, the direction of the movement being downward, the continuation of the vein should be sought in the direction $\mathrm{D}$, or in general. Rule.-If at the point where the vein is lost the Fault-plane lies under foot; to find the continuation of the vein, drive upwards on the Faultplane. If the Fault-plane lies overhead at the point where the vein is lost, drive downward along the Faultplane A B.

Fig. 10 exhibits a Reversed or Overlap Fault. The portion $\mathrm{C}$ has slipped from $\mathrm{D}$ upwards along the fault plane A B. The direction of this movement being the reverse of that of normal faults; and hence the rule given for finding the continuation of the vein gov-

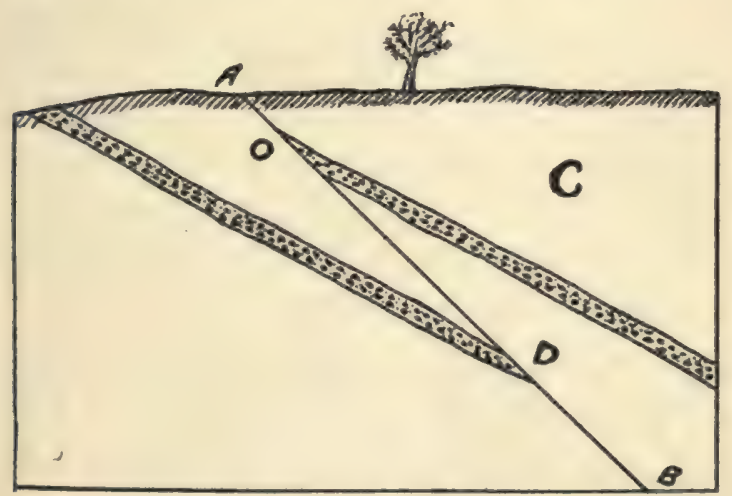

FIG 10.

Reverse Fault. 
erning normal faults, must be reversed in this case. This class of faults are of very rare occurrence in this country, while in the Witwatersrand district, in South Africa, they are not uncommon. In order to determine whether the fault met with is a reversed or a normal fault, the engineer can only determine this by making a careful study of the earth's crust movements of the district in which the fault occurs. It will be observed that if the stratum in the figure were eroded off at $\mathrm{O}$ the outcrop of two veins would apparently be shown, when in fact only one does exist.

Figure 11

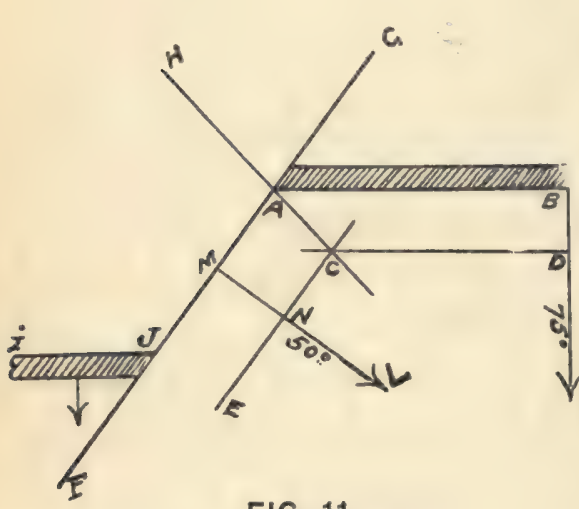

FIG. 11.

Plan of a Faulted Vein.

is the plan of a faulted vein. On driving the drift A B, a fault was met at $\mathbf{A}$. The fault plane was found to dip 50 degrees in the direction $\mathrm{M} \mathrm{L}$, and to rection $\mathrm{AF}$. $\mathrm{AB}$

is the strike of the vein and it dips 75 degrees in the direction $\mathrm{B} K$. It is required to find the continuation or faulted portion of the vein, which is evidently somewhere along the fault plane $\mathrm{G} F$.

Lay off the workings to scale in horizontal section. Assume any convenient vertical depth, say, 100 feet, then $100 \div$ Tan. $75^{\circ}$ equals the distance B D. At D draw the line D C, parallel to A B. In like manner $100 \div$ Tan. $50^{\circ}$ equals the distance $\mathrm{M} \mathrm{N}$. At $\mathrm{N}$ draw the 
line $\mathrm{E} \mathrm{C}$, parallel to $\mathrm{F} \mathrm{G}$, and produce it till it intersects $\mathrm{C} \mathrm{D}$, at $\mathrm{C}$. Through $\mathrm{A} \mathrm{C}$, draw the line $\mathrm{C} \mathrm{H}$, then C A, is the horizontal projection of the intersection of the fault plane with the plane of the vein. Hence, Rule: The heaved part of the vein should be looked for on that side on which the plane of the intersection makes tho larger angle with the plane of the fault, or in the above case at I $\mathrm{J}$, since angle $\mathrm{H} \mathrm{A} \mathrm{F}$, is larger than $\mathrm{G} \mathrm{A} \mathrm{H}$.

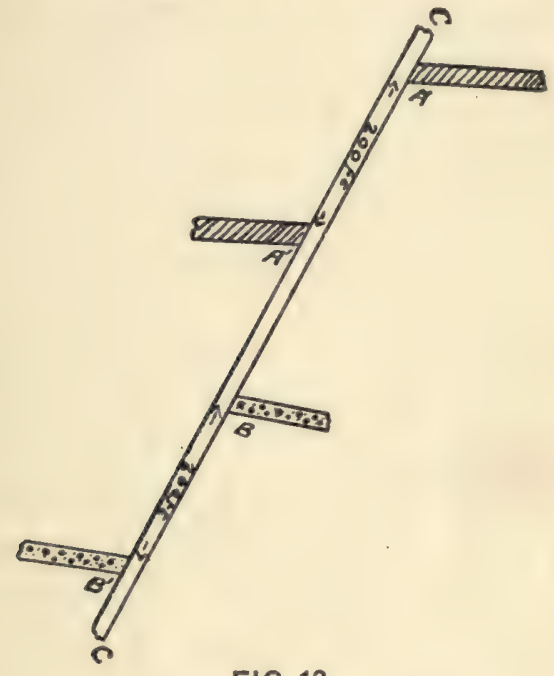

FIG 12.

\section{Veins Faulted By a Dike.}

In Fig. 12, $\mathrm{C} \mathrm{C}$, represents a dike faulting the veins $A$ and B. If the fault has been encountered at $\mathbf{A}$, to find the heaved portion, proceed as in the last case. Now, if in another working the vein is lost at $B$, this vein having been faulted by the same dike $\mathrm{C} \mathrm{C}$, it is evident that the horizontal displacement $\mathrm{BB}^{\prime}$ is equal to the distance A A'. If the horizontal displacement or heave A $\mathbf{A}^{\prime}$, is say, 200 feet, then the heave B B' will also be found to equal 200 feet and the same amount of horizontal displacement may be expected of all other veins intersected 
by the dike $\mathrm{C} \mathrm{C}$; the movement always being in the same direction.

Nole-If C C is a vein, it is evident that it is younger than the veins through which it cuts. In this case it would be called a Cross-Course.

\section{THEORIES.}

The most popular theories which claim to account for the filling of metalliferous veins are:

(a) Theory of Ascension.-This theory supposes veins or lodes to have been formed in part only of minerals dissolved out of rocks in the immediate horizon of vein fissures, and that the chief portion of the material has been derived from greater depth by solvents circulating through the fissure and subsequent precipitation of the minerals in solution on the walls of the cavity.

(b) Theory of Laterul Secretion.-This theory teaches that water perculating through the country rocks has by the aid of carbonic acid and other natural solvents, dissolved out of it all the material now forming the constituents of mineral veins.

(c) Theory of Sublimation.-According to this theory vein fissures were filled by the volatilization of metalliferous minerals derived from the ignited interior of the earth. This theory is fast losing its advocates.

(d) Theory of Replacement.-This is one of the most recent theories promulgated, and it has been much discussed of late. This theory claims that the metalliferous contents of deposits were obtained metasomatically, that is, there were a molecular substitution of the minerals contained in the circulating waters for particles of the wall rocks. Thus the interchange was atom for atom until the walls of the fissure were impregnated with the metalliferous substances which now form the ore deposits. (See Fig. 7).

"Ore Deposits of the United States and Canada," by J. F. Kemp. 


\section{THE EXAMINATION OF MINES.}

\section{VALUATION.}

The object of making mining examinations is to determine as nearly as possible the real and prospective value of mining properties. The real value is arrived at by making accurate surveys, samplings, assays, tests and estimates of the ore exposed or in sight. The prospective valuation of the property, of course, will depend upon surrounding conditions, viz: the locality, nature and probable extent of the ore deposit, richness of the ore exposed, cost of operation, etc.

In any case the party making the examination should be governed by the facts in the matter as they are encountered in the course of his examination of the property, and his report on the mine or prospect should fully explain how these facts were arrived at. If a mining property cannot be (without further exploration of its ore bodies) profitably operated, it should not be reported on as a mine but as a prospect.

METHOD OF ESTIMATING THE NUMBER OF TONS OF ORE IN SIGHT.

According to a custom in vogue among mining engineers the ore in the mine must be blocked out so that at least two or three sides of the ore bodies shall be exposed before any estimate of the ore in sight can be made. Where three sides are exposed, their dimensions are accurately taken according to the surfaces sampled, and the cubical contents of the ore masses thus sampled are accurately computed. Where two sides only are accessible to the termini of the consecutive working levels (which are generally 100 feet apart on the dip of the vein) above and below are jointed together by imaginary lines, thus dividing the ore masses up into triangular solids. The parts exposed are accurately measured 
and sampled, and the cubical contents of each triangular solid computed and recorded. Where only one side of the unblocked ore is shown no estimate of ore in sight of that part of the mine can be made. Figure 13 repre-

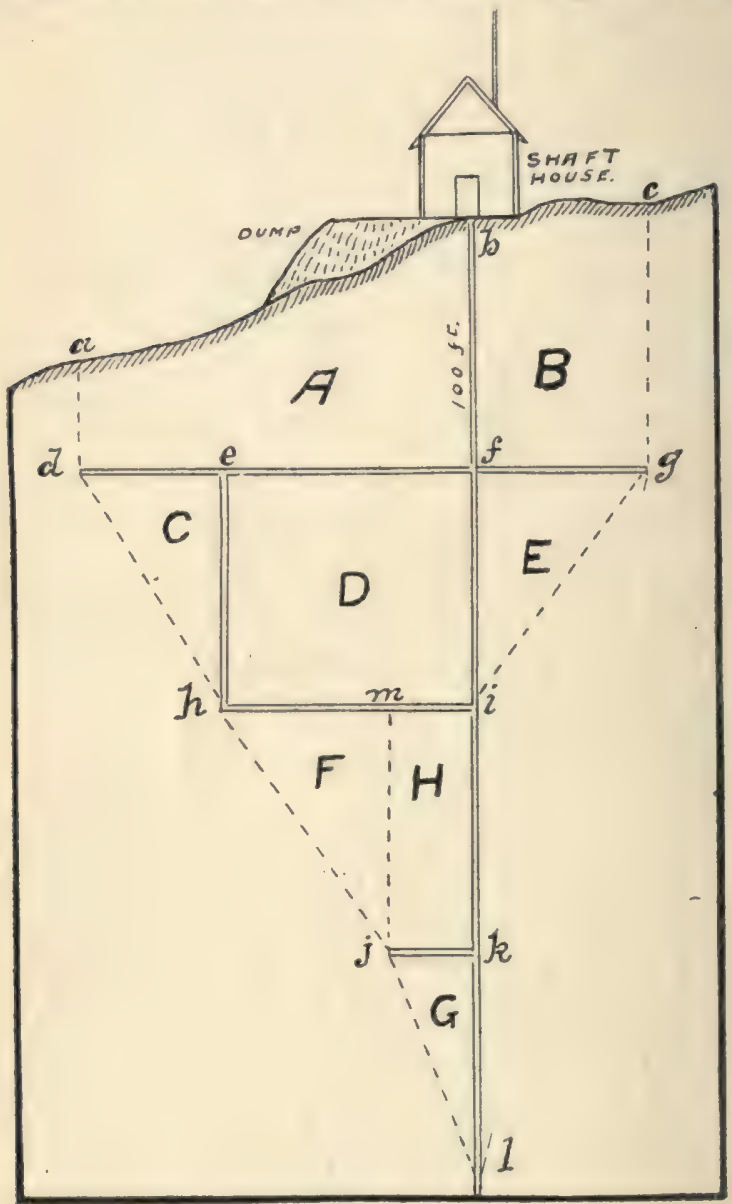

FIG 13.

Longitudinal Section Illustrating Method of Sampling and Estimating Ore in Sight.

sents a longitudinal, and Figure 14 a cross section of a partly developed mine. In Fig. 13, $a b c$ is the surface outcrop of the vein, $b l$ the incline shaft sunk on the dip 
of the vein, and $d g h i$ and $j k$ are drifts driven on the strike of the vein.

To estimate the total tonnage of ore in sight of this mine proceeds as follows: Sample the three sides $a b, b f$ $f d$, calculate the superficial area in feet of the side

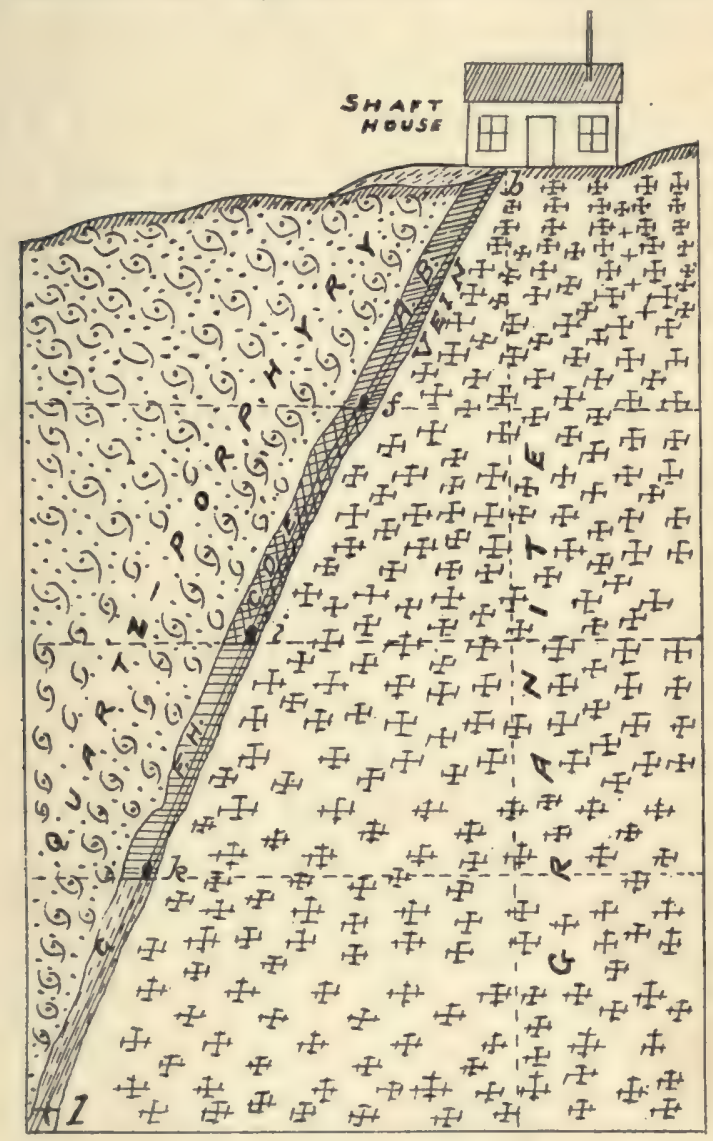

FIG. 14.

Cross-Section Through Shaft bl Showing Formation, Class of Ore Deposits, Width and Dip of Vein.

$a b f d$ and multiply this area by the average width of vein in feet (as determined from the several measurements made when sampling the block) ; the product equals the 
number of cubic feet of ore in block A. In like manner determine the cubical contents of all other sections of which three sides have been sampled and measured, as $B, D$, and $H$. The two sides respectively of the triangular figures $\mathrm{C}, \mathrm{E}, \mathrm{G}$ and the portion $m h$, are next sampled, measured, and the cubical contents of each triangular mass determined as above.

Having the sum of the several ore masses in terms of cubic feet, to calculate the number of tons (of 2,000 pounds) of ore in sight. Rule: Divide the total number of cubic feet in the deposit by 32, and multiply the quotient by the specific gravity of the ore; the product equals the tonnage sought.

In practice it is customary to estimate the ore of which only two sides have been sampled separately from that of which three sides were shown. When sampling a mining property it should be borne in mind that the richer ore generally lies in chutes. These chutes are diagramed out and their tonnage estimated independent of the poor ground or intervening low grade ores. In any event, the method of sampling and computation adapted should be fully explained in the report.

Note.-The more general rule among engineers and mine operators is that three sides, at "least," of the ore body shall be sampled before an estimate of ore in sight can be made.

\section{SURVEY AND MAPS.}

If reliable maps and drawings of the mine are not at hand, about the first thing to do is to make a survey of both surface and underground workings and from this survey prepare a map of the claims, a plan of the mines and at least two sections of the workings. On these drawings in both plan and sections should appear the numbers and position of all samples taken. 


\section{SAMPLING.}

In sampling trenches should be cut across the vein or deposit (if a vein at right angles to its strike and dip) at not less than five (5) feet apart, and average samples of the ore taken therefrom; a section of the place sampled should be sketched in the note book, together with a full description of each sample taken. From this data we determine the amount of ore in sight and subsequently its average value per ton.

\section{CLASSIFICATION OF ORES.}

High grade ores should be sampled and estimated separately from the lower grade ores, and in many cases it is necessary to divide the ores into first, second and third classes.

\section{MILL RUN SAMPLES.}

If a mill run of the ore is required, samples are taken, so that their total weight shall amount to one or more tons, and these should represent a fair average of all ore bodies sampled.

\section{LABORATORY TESTS.}

In any case it is all important that the engineer make laboratory or mill tests, with a view of arriving at the actual per cent. of concentrates contained in the ore, The assay value of concentrates and the approximate amount of ore in sight which would require concentrating should receive careful consideration. Free milling tests, amalgamation assays, cyanide and chlorination tests, etc., should also be made in order to decide upon the most economic process of treating the ore.

\section{ENGINEERS' DUTIES.}

All samples of ores taken, assays of samples, laboratory tests of ores, estimates of value and number of tons of ore in sight, investigation of title to property, etc., should be made in person by the engineer in charge 
of the examination. If, through negligence, inability, or other causes, these requirements are not fulfilled his report on the property should not be esteemed worthy of consideration, and moreover it should here be borne in mind that the mere opinion of the so-called Mining Fixpert generally counts for nothing, unless his conclusion in the matter derives from a well conducted examination of the property.

\section{PRECAUTIONS.}

It is a grave mistake to recommend the erection of reduction works of any kind until such time when it shall have been thoroughly demonstrated beyond a doubt that the ore, from both an economic and metallurgical standpoint, is best suited to the process recommended and the merit of the mine well established, with ore enough blocked out to fully warrant and justify such expenditures.

Having completed the examination of the mine, the next step is to draft the report. The following is a general outline of a mining report, which may be modified to suit any particular case:

$$
\begin{gathered}
\text { REPORT ON } \\
\text { THE MINING CO'S PROPERTY. }
\end{gathered}
$$

To

Gentlemen:- Pursuant to your request, I have made a very thorough examination of your mines, situated at and herewith submit the following report:

GEOGRAPHY.

(1) Locality and key map of country? (2) Altitude? (3) Accessibility? (4) Railway fare and transportation rates? (5) Wagon roads, etc.? 
GENERAL DESCRIPTION OF PROPERTY.

(1) Name of claims, and area of group? (2) Map of claims? (3) Abstracts of title? (4) Surface property and improvements? (5) Mining and milling machinery? (6) Climatic conditions?

HISTORY.

(1) Output of district, $\$ \longrightarrow$ ?

(2) Total number of tons of ore extracted from mine, and treated by the process? (3) Average assay value of ore mined and treated by the - process at

(4) Cost per ton of mining ore? Cost per ton of milling ore by the process and per cent. of assay values saved? (6) Transportation cost? ( 7 ) How above information regarding the past history of the mine was obtained?

\section{GEOLOGY.}

(1) Formation of district? (2) Foot wall of ore deposits of mine? (3) Hanging walls? (4) These ore deposits are of the class? Gangue of ore? (6) An average analysis of all samples taken gave — per cent. — per cent. - per cent. $\$$

(7) (Here introduce)

Plan of Mine? (9) Longitudinal section and two or more geological cross sections of the underground workings and on the above drawings should appear the numbers and position of all samples taken? (10) Number of feet of development, shafts, drifts, _ $\mathrm{ft}$., crosscuts, — ft., winzes, _- ft., upraises, _ $\mathrm{ft}$., cubic $\mathrm{ft}$. of ground stoped out

\section{ORE IN SIGHT AND ASSAY VALUES.}

(1) List of assays of all ores sampled? (Give number of each sample, width of vein, with a sketch of each sample showing place where taken) (2) Total number tons (of 2.- 
000 pounds) of ore in sight (explain by drawings how estimated), — tons. Of this — tons, is first-class ore, which will average $\$$ per ton. Total value of all first-class ore in sight is $\$$ - (3) Of the remained (second-class ore) there are —_ tons, which will average $\$-$ per ton. Total value of all second-class ore in sight, \$—. (4) Grand total value in dollars (according to assays and present market value of metals) of all ores in sight is \$—. (5) The amount of concentrates in the first-class ore will average — per cent. of its total weight, and represents - per cent. of its total value, and that of the secondclass ore is _- per cent. of its weight and represents - per cent. of its assay value.

ESTIMATE COST OF EXTRACTION AND REDUCTION OF ORE IN SIGHT.

(Cost per ton.) (1) Mining $\$$. Transportation $\$$ —. (3) Treating ore by the process at — mill \$—. (4) Loss in treatment $\$$ —. (5) Refining, assaying and marketing bullion $\$$ _. (6) All other items of expense, including interest on capital invested, say $\$-$ per ton. (7) Total cost of extraction and reduction $\$-$ per ton.

The net profit on all ore treated (taking the average assay values of the ore as a basis) is, therefore, $\$$ - per ton. (9) The mine at present is capable of supplying - tons of ore per day, for — time, which would mean a profit of $\$$ - per month (of 26 working days), or $\$$ for months.

PROSPECTIVE OUTLOOK OF MINE AND COST OF DEVELOPMENT.

(1) List of assays of samples taken, where less than three sides of ore were exposed? (As before, give sketch of sample, etc.) (2) Cost of sinking shafts 
$\$-$ per foot? (3) Driving drifts $\$$ - per foot?

(4) Crosscuts $\$ —$ per foot? (5) Winzes $\$-$ per foot? Upraises, etc., \$- per foot?

MINING AND MILLING FACILITIES.

(1) Lumber costs \$— per_—? (2) Cord wood $\$ —$ per cord? (3) Coal \$_ per __ ? (4) Mining timbers \$— per — ? (5) Labor (classify) $\$$ ? (6) Freight rates from — to mine \$per_—? (7) Water supply (describe)? PRICE AND TERMS OF SALE.

(State these conditions clearly and in as few words as possible.)

GENERAL DESCRIPTION OF ADJOINING PROPERTIES.

(Describe briefly such properties as have a bearing on this examination.)

\section{RECOMMENDATIONS.}

Under this paragraph should appear such recommendations as in the engineer's judgment are appropriate and essential to successful and economic operation of the mine. The reduction process to which the ores of the mine have proven best adapted should receive careful consideration.

CONCLUSION.

(The conclusion here should be drawn from the foregoing facts as they appear in the report and not from the imagination, as is too often the case with mining reports.) 


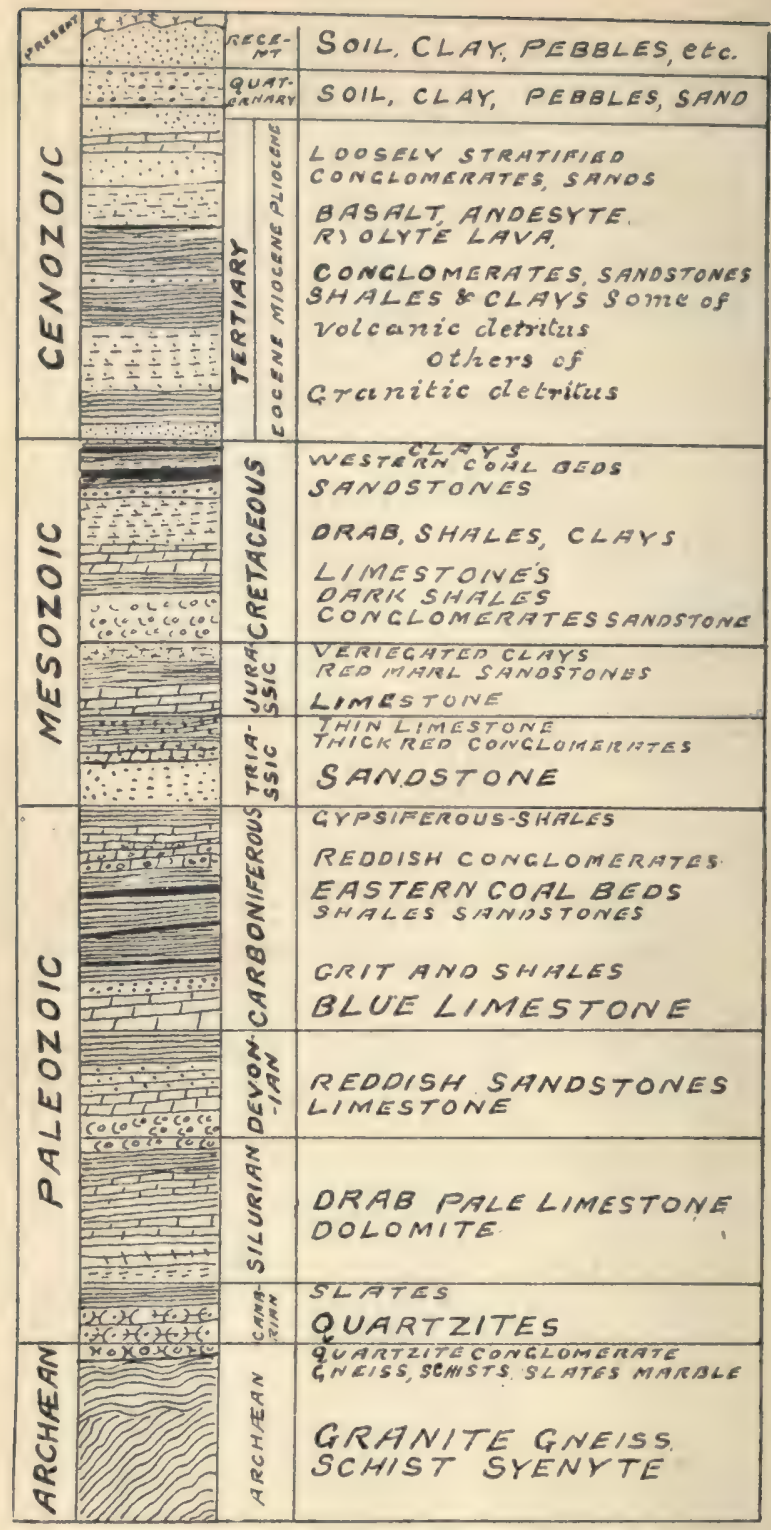

\section{WESTERN GEOLOGICAL SECTION.}

(After Prof, Lakes in part.)

Showing the General Order of Formation as they Cccur on the Pacific Slope and Rocky Mountain Regions. 


\section{PART II.}

\section{Chemical Operations}





\section{PART II.}

NOTES ON ASSAYING GOLD, SILVER, LEAD AND COPPER ORES.

GOLD AND SILVER.

Crucible Process for the Assay of Gold and Silver Ores.

Pass the ore through a sixty or eighty mesh sieve, and:-

(a) If the ore is silicious with little or no sulphides, etc., use the straight formula, as given below.

(b) If the gangue of the ore consists of oxides, lime or calciti, etc., add to the charge $1 / 2$ assay-ton (A. T.) of silica.

(c) If the ore is a sulphide or concentrates, or if it contains arsenic, antimony, etc., it should be thoroughly roasted. Weigh out before roasting ore $1 / 2 \mathrm{~A}$. T., mix with an equal volume of charcoal and $1 / 2 \mathrm{~A}$. T. silica, roast in a chalk-lined frving pan at a low heat with continual stirring.

GENERAL FORMULA.

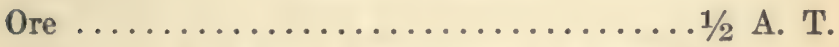

Lead Flux $\left\{\begin{array}{l}\text { Sodium bicarbonate, } 16 \text { parts } \\ \text { Potassium carbonate, } 16 \text { parts } \\ \text { Borax glass, 8 parts } \\ \text { Flour, } 4 \text { parts }\end{array}\right\}$ A. T.

Soda bicarbonate .................... A. T.

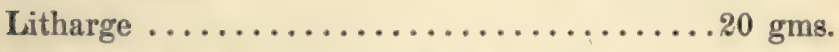

Transfer this charge well mixed to a crucible (which should not be more than three-fourths filled), cover with salt and fuse in the muffle furnace at a high 
heat for about forty minutes, or at least until all action of the charge has ceased. Then pour into the moulds, let cool, detach lead button, hammer into a cube and cupel at a bright red heat until all the lead is gone and the remaining gold and silver globule brightens; now brush, flatten and weigh, calling the weight of button gold and silver two ounces for each milligramme it weighs; note the weight, say it $=20 \mathrm{ozs}$.

NOTE.-If the button does not contain at least twice as much silver as gold, before trying to part, that amount of pure silver should be fused with it.

With dilute nitric acid, in a small porcelain capsula, or crucible, dissolve the button over a spirit lamp. The gold falls to the bottom as a dark scale or powder; renew the acid and finely wash with water, pour off the water and remove the last drop with a piece of clean blotting paper, dry the crucible, with its gold contents, very thoroughly, cover and transfer the crucible or capsula to the heated muffle for a few minutes, then let cool and weigh as metallic gold, say this weight $=1.65$ ozs. Then 20 ozs. -1.65 ozs. $=18.35$ ozs.; that is, there are 18.35 ozs. of silver to each ton of 2,000 pounds and 1.65 ozs. of gold. Taking gold at $\$ 20$ per oz. and silver at $65 \mathrm{c}$ per oz., we have for the assay: Gold $\$ 33.00$ and silver $\$ 11.93$, or a total value of $\$ 44.93$ per ton of ore.

\section{LEAD.}

Crucible Process for the Assay of Lead Ores.-In case of sulphides, such as galena, pyrite, etc., insert in the crucible with the charge three iron nails (eightpenny), points down. These should be removed rapidly, but carefully, after the fusion is complete. Pass the ore through an eighty mesh sieve and weigh as below. 
GENERAL FORMULA.

Ore ..................10 grammes

Soda-Bicarbonate .........15 grammes

Carb. Potassium ...........10 grammes

Argol ................ 7 grammes

Flour ............... 5 grammes

Borax Glass .............. 3 grammes

Salt cover, and three iron nails if a sulphide ore. Run at a moderate heat for from twelve to fifteen minutes, or to complete fusion; then remove the crucible, take out nails, cover as rapidly as possible, and when stone cool break the crucible, detach lead button and hammer into a cube. The weight of button in grammes multiplied by ten, gives the ore's percentage of metallic lead.

COPPER.

Wet Process for the Assay of Copper Ores.-(1) Treat one gramme of the finely pulverized ore in a (250cc capacity) flat-bottomed flask or casserole, with 7ce of nitric acid, 5cc sulphuric acid, and if the ore is a heavy sulphide add one gramme of potassium chlorate (after boiling). Heat until all red fumes are gone, or until the nitric acid has been driven off, and all the copper is in solution. Now let cool and add seven grammes pure zinc, cut into thin strips, and $50 \mathrm{cc}$ of water. Shake the contents of the flask in order to break up any cake formed in the bottom and allow to stand fifteen minutes, then add 50cc water and $10 \mathrm{cc}$ sulphuric acid. Let cool thoroughly. The copper has been precipitated as metallic copper. (2) The solution of zinc having been completed, fill up to the neck with water, allow to settle for about five 
minutes and decant or pour off the clear supernatant liquid. Fill with water and decant in the same manner twice more. There remains in the flask the insoluable residues along with the metallic copper, etc. (3) To the insoluable residue (silica) and metallic copper in the flask, add 5cc of nitric acid, boil for about five minutes or until all red fumes have gone, let cool, then add $10 \mathrm{cc}$ of ammonia, or enough to neutralize the acid. The solution now turns blue in proportion to the amount of copper present. (4) When the solution is cool add 50ce of water, and in case of rich ores (above five per cent. ore) runs into the flask from the burette enough of the standardized solution of cyanide of potassium to almost bleach the solution in the flask; having noted the amount of cyanide solution used, filter off the silica, etc., catching the light blue filtrate in a beaker. Now run from the burette into this filtrate enough cyanide solution to bleach or discolor it. Note the total amount of cyanide solution used, say in this assay $20 \mathrm{cc}$ were required.

NOTE.-The Potassium Cyanide Solution is prepared as follows: Dissolve sixty grammes of the cyanide salt in one litre of water, when dissolved decant or ciphon the solution into a paper wrapped-bottle. The solution is now ready for standardizing; this is done as follows: Weigh out say 250 milligrammes of pure copper and in a beaker, dissolve this in 5cc nitric acid, boil until red fumes have disappeared, let cool, then add $10 c c$ of ammonia, let cool and add 50cc of water. Then run in from the burette enough of the previously prepared cyanide solution to bleach or discolor the copper solution in the beaker; note the amount used, say in this case $15.15 c c$ were required; then $1 c c$ of cyanide solution $=250 \div 15.5=16.13$ milligrammes of copper, or since 1000. mgrs. $=1$. gramme, 16.13 mgrs. $=.01613$ grammes. Therefore, 1ce of the cyanide solution will neutralize or 
indicates the presence of .01613 grammes of pure copper.

Now, since in the assay of the ore, 20ce of the cyanide solution were used, it is evident that there is present $.01613 \times 20=.32260$, or 32.26 per cent. of pure copper in the ore.

Note.-This .01613 is called the standard factor and it should be labled on the cyanide solution bottle for future reference.

If two litres of water, instead of one, or 30 grammes of the cyanide salt, instead of 60 grammes, had been used in making up the solution the standard factor would have been $.01613 \div 2=.00806$. A solution of the latter strength is preferable, since the error in reading the burette is only one-half of that in the former case. In practice, where a great number of assays are to be made at one time the most convenient solution is one of which $1 \mathrm{cc}=.01$ grammes, or 10 milligrammes of copper, or which is the same thing, 1ce of cyanide solution equals 1 per cent. copper. Such a solution may be made by diluting the above solution to 1.613 litres of water.

Note.-The strength of the solution will depend upon the purity of the cyanide salt used.

\section{SECOND METHOD.}

If there is no zinc, nickel or cobalt present in the ore, the following rapid method is preferable and in general gives more satisfactory results: Take ore, 1 gramme, and boil in flask as before with $7 c c$ nitric acid, 5ce sulphuric acid and about 1 gramme potassium-chlorate added a little at a time (if the ore is a heavy sulphide), boil until the red fumes have cleared away (or until all the copper is in solution), then let cool and add 15ce of ammonia (or enough to neutralize the acid) and 50ce water, let cool and run in enough cyanide solution to almost bleach the solution 
in the flask or beaker (note amount of solution used). Now filter and finish the titration. As before, multiply the standard factor representing the strength of the cyanide solution by the number of cubic centimeters of solution used; this product multiplied by one hundred gives the ore's percentage in metallic copper.

Note.-The final addition of cyanide solution should be added from the burette drop by drop, the flask containing the assay being well shaken each time until the blue or lilac tint can scarcely be discerned at the upper edge of the liquid when viewed against a white background. Many chemists titrate to a faint rose or pink tint (Furman).

\section{LABORATORY TESTS OF GOLD AND SILVER}

\section{ORES.}

OBJECT OF TESTS.

The following tests of gold and silver ore are made in order to determine the probable percentage of gold which may be extracted by the cyanide, chlorination and amalgamation processes. In testing by the cyanide process it should be borne in mind that the least amount of cyanide which it is possible to use, and yet extract all the gold, the more economic will be the process of treatment. In general, the finer the ore is crushed and the longer that it is leached, the greater will be the per cent. of extraction. The percentage of gold extraction by the chlorination process will depend not only upon the fineness of crushing but also upon the per cent. of free gold present, the amount of chlorine gas generated per ore charge and the time of agitation. Amalgamation tests, or assays, are made on gold and silver ores to determine the probable per cent. of gold and silver which may be extracted by the amalgamation process of milling. 
TESTING BY THE CYANIDE PROCESS.

Crush the well selected sample and pass it through at least a forty mesh sieve. Weigh out 500 grammes of the ore, shake with water, and insert a blue strip of litmus paper; if the litmus paper turns red an acid is present which must be removed. Neutralize the acid with lime by adding a little at a time and continue until the red litmus turns blue. Note the weight of lime required to neutralize the 500 grammes of ore. Wash the ore thoroughly with water, dry and weigh out four samples, each 100 grammes, and pour these into wide-necked unstoppered flasks which label No. 1, 2, 3, 4 , respectively. Measure out and pour into each flask or bottle, with the samples, $100 \mathrm{cc}$ of water (100 gms.). Now weigh out two lots of the cyanidte salt, each 0.4 grammes. Dissolve one lot with sample No. 1, the other lot with sample No. 2. Now 0.4 gms. : 100 gms, :: 8 lbs. : 2,000 lbs.; that is for each ton of water used in the milling process eight pounds of cyanide will be required, or, since in practice one ton of ore is leached in one-half ton of water, one ton of ore would therefore be treated with four pounds of cyanide. Let No. 1 leach for, say twelve hours, and No. 2 for twenty-four hours, then filter off one assay ton $(29.17 \mathrm{cc})$ of each solution and evaporate to dryness in a lead foil dish, made out of pure lead for the purpose, and when evaporation is completed fold up lead dish and cupel; treating the resulting button as in the regular assay of gold and silver ores. The difference between this assay and that of the ore before treating equals the amount of loss in treating. Nos. 3 and 4 are tested in like manner, but for greater lengths of time, and with weaker solutions. The loss of liquid in the bottles due to evaporation should be estimated and allowed for in the assay. As a check on 
the work, wash and dry samples and assay leached pulp. The difference between the assay of the leached pulp and the assay of the ore before treatment should equal the amount of gold recovered, or in solution. An approximate determination of the amount of cyanide consumed per ton of ore may be made as follows: Dissolve in two litres of water fourteen grammes potassium-iodide and seven grammes metallic iodide. Dissolve one gramme of cyanide in one litres (1,000 gms.) of water; this corresponds to two pounds of cyanide to the ton of water. Measure out $30 \mathrm{ce}$ of this cyanide solution, add a few drops of starch solution (starch rubbed and dissolved in water) and titrate with the iodide solution until a bluish yellow color is seen. Now divide the two pounds (cyanide in one ton of water) by the number of (cc) iodide solution required or used, the quotient indicates the number of pounds of cyanide per ton of water that each (cc) of the iodide solution is equal to. 'This quotient is called the standard factor and it should be labeled on the iodide solution bottle for future reference. After agitating the assays in the bottles with zine shavings for a few minutes, filter and measure out $30 \mathrm{cc}$ of the solution, titrate as above and multiply the number of (cc's) of the iodide solution used by the standard factor, the product is equal to the number of pounds of cyanide in a ton of the solution tested. Knowing the strength of the cyanide solution before and after using, the difference, therefore, equals the loss in treatment, or the amount consumed. In cyanide mill practice it is customary to leach the ore first with the strongest solution for the proper time, then with a weaker solution. After this it is washed with water, running all solutions after being filtered through boxes filled with zinc shavings. In these boxes the gold and silver is precipitated, 
while the solution, after being freed from its values, is caught in a receiving tank from whence it is pumped back to the standardizing tanks and cyanide added until it is brought up to the proper strength for using again. The above tests should be conducted in such a way as to imitate as nearly as possible the actual operation of the cyanide mill.

TESTING BY THE CHLORINATION PROCESS.

Pass the pulverized sample through a forty mesh sieve, weigh out one pound of the ore and roast, if a sulphide, to a dead roast, introduce the roasted ore into a glass-stoppered bottle containing $300 \mathrm{cc}$ hot water and 6.8 gms. of each, bleaching powder and sulphuric acid $\left(66^{\circ}\right.$ Baume). Agitate the bottle and its contents from four to eight hours, then filter off the solution, wash the pulp with water until the washing no longer gives a precipitate on testing for chlorine with silver nitrate. Now dry the pulp and assay it in the usual way. Having the assay on the ore before and after treatment, the difference represents the loss in treatment. The above test is made on the basis of there being thirty pounds of each-sulphuric acid and bleaching powder-used to the ton of ore in the regular milling process. These proportions should be varied, however, the proper amount of each chemical required can be determined only by experiment. At the end of the process of agitation there should be free chlorine present, which is indicated in the presence of ammonia water by giving off the characteristic fumes of ammonia-chloride.

TESTING BY THE AMALGaMATION PROCESS.

Pulverize the ore and pass it through an eighty mesh sieve. Weigh out $50 \mathrm{~A}$. T., place in an ordinary gold pan and pan down to black sand. Wash the contents into a wide-necked bottle, add two ounces of mer- 
cury and hot water, agitate for a few minutes, then pour off the contents, except the mercury and amalgam, which is washed several times with water. The contents of the flask is now washed into the gold pan and the mercury and amalgam cleaned by further panning. Now strain off the mercury through a piece of tight buckskin. The amalgam caught in the buckskin is collected and placed into a small porcelain crucible, the crucible is heated gradually at first, then to redness after the mercury is driven off. The contents of the crucible, after cooling, is wrapped in a piece of sheet lead and cupelled. The resulting button indicating the value in fifty tons of ore is treated as in the regular assay of gold and silver ores. Having assayed the ore before treatment, the difference between the first and this last assay is equal to the loss in treatment.

\section{BLOWPIPE ANALYSIS OF MINERALS.}

BLOWPIPE OUTFIT.

By this method of tests the composition of minerals are indicated by certain colors, etc., exhibited when heated alone and in the presence of certain regents. In order to successfully conduct such tests the following outfit at least is required:

First. The Blowpipe. Plattner's is the best, the jeweler's or cheaper variety will do where a great number of tests are not to be made.

Second. One alcohol lamp with a tightly-fitting top to prevent leakage when in field use.

Fourth. A small amount of platinum wire, one piece short and another thick, the short piece to be used for stirring the assay, the longer and thinner piece for making the bead tests.

\footnotetext{
"Economic Mineralogy," by Prof. B. Sadtler.
} 
Fifth. Open and Closed Tubes.-These should be made from Bohemian glass tubing of about one-fourth of an inch diameter, cut into four-inch lengths. To make the closed tubes simply fuse one end shut with the blowpipe, using the hotter portion of the flame, which is just beyond the point of the inner blue flame.

Sixth. Plaster and Bone-ash.-When these are difficult to obtain any pure clay well burned and powdered will form a good substitute.

Seventh. A pair of platinum-tiped forceps for making the flame tests and testing the fusibility of min. erals.

Eighth. Two or three Bohemian watch glasses to be used for making the acid tests of the finely powdered mineral, and Ninth. Several lumps of charcoal. This should be well burnt, and fine grained; that of the smaller limbs is generally the best.

Besides the above a number of glass-stoppered bottles containing the following named reagents should be added :

LIST OF CHEMICALS AND REAGENTS.

1. Soda bicarbonate.

2 Powdered Borax Glass.

3. Salt of Phosphorus, SPh.

4. Bismuth-Flux-(2 parts sulphur, 1 part potassic-iodide and 1 part potassic-bisulphate.)

5. Boracic-acid.

6. Boracic-acid flux-(Potassium sulphate, $\mathrm{K}_{2} \mathrm{SO}_{4}$, $41 / 2$ parts and Calcium bifluoride $\mathrm{CaF}_{2}, 1$ part.)

\%. Copper oxide, $\mathrm{CuO}$.

8. Potassium-sulphate $\mathrm{K}_{2} \mathrm{SO}_{4}$

9. Ammonium-sulphide $\left(\mathrm{NH}_{4}\right)_{2} \mathrm{~S}$.

10. Magnesium (metallic).

11. Test Copper (metallic). 
12. Granulated lead.

13. Granulated zinc.

14. Test tin.

15. Powdered Silica, $\mathrm{SiO}_{2}$.

16. Cobalt solution.

17. Ammonia-water, $\mathrm{NH} \cdot \mathrm{OH}$.

18. Nitric acid, $\mathrm{HNO}^{3}$.

19. Hydrochloric acid, $\mathrm{HCl}$.

20. Sulphuric acid, $\mathrm{H}_{2} \mathrm{SO}_{4}$.

21. Potassium-bisulphate.

The above outfit complete with apparatus and chemicals will cost $\$ 10$.

\section{OPERATION AND TESTS.}

(a) THE oxidizing FLAMe. (o. F.).

To produce the $\mathrm{O}$. F., the point of the blowpipe is placed just within the flame of the lamp, slightly above the wick. Blowing produces a long blue cone; the $\mathrm{O}$. F. lies just outside this blue cone and is not often visible unless some object is placed in it. Just beyond the point of this blue cone is the hottest part of the flame.

(b) THE REDUCING FLAME (R. F.).

To produce the R. F., hold the blowpipe so that its point just reaches the edge of the lamp flame slightly above the wick. The R. F. is the interior of the blue cone mentioned above, and it is most active near its point.

\section{(c) FLAME TESTS.}

This test is made by holding a small bit of the mineral in a pair of platinum-tiped forceps or wrapped in platinum wire in the colorless flame just beyond the cone and noting the coloration of flame produced. The platinum tips or wire should be cleaned between tests. 


\section{(d) TESTS WITH REAGENTS.}

In the examination of sulphides, arsenides, antimonides and related ores, the assay should be roasted before using a flux, in order to convert the substance into an oxide. This is done by spreading the substance out on a piece of charcoal and exposing it to a gentle heat in the O. F. The sulphur, arsenic, antimony, etc., pass off as oxides in the form of vapor, leaving the non-volatile metals behind as oxides. The escaping sulphurous acid gives the ordinary odor of burning sulphur, arsenic acid, from arsenic present, the odor of garlic, selenious acid, from selenium present, the odor of decaying horseradish, while antimony fumes are dense white and have no odor.

After the finely pulverized mineral is roasted as directed above, take a piece of the thinner patinum wire, make a loop in one end of it about the eighth of an inch in diameter. Now heat the loop in the blowpipe flame and dip into the dry borax, soda or S. Ph., as the case may be, the adhering material fused, and again dipping into the reagent and fused until a clear bead is formed within the loop. Now fuse the bead again, and this time dip it into the finely powdered mineral; fuse this and dip again, noting each time the colors which the bead assumes, both when hot and cold. Care should be taken not to take too much of the mineral at a time, it being better to add it to the bead in minute quantities until the desired depth of color is obtained. In selecting the mineral to be tested, care should be taken to not confound two or more minerals together as this often causes unnecessary conflicts in tests.

(e) TESTS ON OHARCOAL.

Mix the pulverized mineral and reagent (slightly moistened) together in a little round 
3all, place in a shallow orifice or cone-shaped cavity made in the charcoal. Now play upon it with the desired flame, and note the kind of colors forming the coatings on the coal around the assay, also note the color of bead as before, the escaping fumes and their odor and whether or not a globule of metal would be given out with further blowing.

(f) TESTS ON PLASTER OR BONE-ASH.

In case of the presence of lead or other heavy metals in large quantities, a prepared surface of dried clay or bone-ash may be advantageously substituted for the charcoal, but whether the test be made on coal or plaster, if the mineral is not previously roasted, the desired colorations are rendered obscure in the presence of volatile substances.

( $g$ ) TESTS IN Closed TUBE (MATRASS).

In closed tube, either heated directly over the Bunsen burner, or in the blowpipe flame, volatile substances of the assay within the tube are vaporized and condensed in the upper or colder part of the tube, where they may be examined by a lense, if necessary, or by further heating. The odor given off may be noted; also the acidity of any fumes by inserting a small strip of litmus paper in the mouth of the tube. Acid fumes turn blue litmus red, and alkalies turns red litmus blue. The closed tube is used to observe all the effects that may take place when the mineral is heated out of contact with the air. The substance, of course, must be finely pulverized before going into the tube and only a minute quantity taken.

(h) TESTS IN THE OPEN TUBE.

This tube being open at both ends the atmosphere passes through in the heating and so modifies the result. The assay is placed 
an inch or an inch and a quarter from the lower end of the tube; the tube should be held nearly horizontal or enough so as to prevent the substance from falling out. The assay is now heated over a Bunsen burner or with the blowpipe flame and its action noted. (See 'Tests.)

It is believed that the above is all that is necessary to enable the student to successfully conduct the Blowpipe tests given on page 54 to 81 .

QUALITATIVE TESTS OF MINERALS IN THE WET-WAY.

By the following method of tests, the mineral to be tested is first pulverized to a very fine powder, a small portion of it, or about as much as can be held on the point of a large pocket-knife blade is dissolved by boiling with about 5cc to $10 \mathrm{cc}$ of the proper acid, or acils; in a test tube, matrass, or beaker. The acids used as solvents are generally Hydrochloric $(\mathrm{HCl})$, or Nitric $\left(\mathrm{HNO}_{3}\right)$, or both. In no case should an acid be used which contains the element sought for in the test, or when its presence would interfere with the test, for instance, as in the test for silver, $\mathrm{HCl}$ should not be used as a solvent, "but as a precipitant." (See silver.)

The precipitates, in many cases, after being caught upon the filter, may be dried and further tested by means of the blowpipe; thus confirming by blowpipe tests the presence of the element sought. The acids used as solvents in the majority of cases should be diluted with water or at least before applying the respective precipitants, i e, the reagents or chemicals, which indicates the element tested for, if present. Having the mineral thoroughly dissolved, a drop or two of the solution is removed by means of a glass rod or tube, and tested on a white porcelain dish with the proper reagent or chemical added, "as per Tests." In order to suecess- 
fully conduct all tests as given below, small glass-stoppered bottles containing the following reagents and chemicals are required. They may be procured from the various dealers in chemical supplies, and ordered as needed:

LIST OF CHEMICALS, REAGENTS, ETC.

(1) $\mathrm{NH} \cdot \mathrm{OH}-$ Ammonium Hydrate.

(2) $\mathrm{NH} \cdot \mathrm{Cl}-A m m o n i c$ Chloride.

(3) $\mathrm{NaC}_{2} \mathrm{H}_{3} \mathrm{O}_{2}-$ Sodic Acetate.

(4) $\mathrm{H}_{2} \mathrm{~S}-$ Sulphuretted Hydrogen. (Prepared by adding dilute Sulphuric acid to pure iron sulphide. The gas thus generated should be washed by passing it through water before using.)

(5) $\mathrm{HCl}-\mathrm{H} y d r o c h l o r i c$ Acid.

(6) $\mathrm{AgNO}_{3}-$ Silver Nitrate.

(7) $\mathrm{KOH}-$ Potassium Hydrate.

(8) $\mathrm{NaOH}$-Sodium Hydrate.

(9) $\mathrm{Sb}_{2} \mathrm{O}$ - Antimony Teroxide.

(10) $\mathrm{Sb}_{2} \mathrm{O}_{5}-$ Antimony Pentoxide.

(11) CuSO-Copper Sulphate.

(12) $\mathrm{HNO}_{2}-$ Nitric Acid.

(13) $\mathrm{H}_{2} \mathrm{SO}-$ Sulphuric Acid.

(14) ( $\left.\mathrm{NH}_{4}\right)_{2} \mathrm{~S}-A$ mmonium Sulphide.

(15) $\mathrm{SnCl}-\mathrm{Tin}$ Bi-chloride.

(16) KCN-Cyanide of Potassium.

(17) $\mathrm{MnO}_{2}-$ Manganese Dioxide.

(18) $\mathrm{BaCl}-$ Barium Dichloride.

(19) $\mathrm{CaCl}_{2}-$ Calcium Dichloride.

(30) Turmeric Paper (Indicator).

(21) Litmus Paper (Indicator).

(22) Metallic Zinc.

(£3) $\mathrm{PbO}$-(Litharge) Lead Oxide.

(24) $\mathrm{H}\left(\mathrm{C}_{2} \mathrm{H}_{3} \mathrm{O}_{2}\right)-$ Acetic Acid.

(25) $\mathrm{H}_{2} \mathrm{O}_{2}-\mathrm{H} y$ drogen Peroxide. 
(26) K.FeCyo-Potassium Ferrocyanide.

(27) $\mathrm{H}_{2}\left(\mathrm{C}_{4} \mathrm{H}_{4} \mathrm{O}_{6}\right)-$ Tartaric Acid.

(28) $\mathrm{H}_{3}\left(\mathrm{C}_{6} \mathrm{H}_{3} \mathrm{O}_{7}\right) \mathrm{H}_{2} \mathrm{O}$-Citric Acid.

(29) Fe-Metallic Iron.

(30) Zn-Metallic Zinc.

(31) $\mathrm{Fe}_{2} \mathrm{O}_{2}-$ Iron Sesquioxide.

(32) $\mathrm{NH}_{4} \mathrm{CNS}-A$ mmonium Sulpho Cyanide.

(33) Nitrophenic Acid.

(34) $\mathrm{Na}_{2} \mathrm{CO}-$ Sodium Carbonate.

(35) NasHPO $-H y d r o d i s o d i c ~ P h o s p h a t e$, (Solution equals $1 \mathrm{gm}$. $\mathrm{Na}_{3} \mathrm{HPO}$, dissolved in $10 \mathrm{cc}$ of water).

(36) $\mathrm{Pb}_{2} \mathrm{O}$ - Peroxide of Lead.

(3\%) $\mathrm{Cu}-$ Metallic Copper.

(38) ( $\left.\mathrm{NH}_{4}\right)_{2} \mathrm{CO}_{3}-$ Ammonium Carbonate.

(39) $\mathrm{NaClO}_{3}$-Sodium Chlorate.

(40) $\mathrm{FeSO}_{4}$-Ferrous Sulphate.

(41) Magnesia Mixture $=\mathrm{MgSO} * 1 \mathrm{gm} ., \mathrm{NH} \cdot \mathrm{Cl}$ $1 \mathrm{gm}$. and $\mathrm{NH}+\mathrm{OH} 4 \mathrm{cc}$, added to $8 \mathrm{cc}$ water. (Dissolve well).

(42) $(\mathrm{N} \mathrm{H})_{2} \mathrm{MoO}_{4}+\mathrm{HNO}_{2}-$ Molybdate Solution $=1 \mathrm{gm} . \mathrm{MoO}_{3}$ dissolved in $4 \mathrm{cc} \mathrm{NH}_{4} \mathrm{OH}$, and the solution poured into $15 \mathrm{cc}$ of $\mathrm{HNO}_{3}$ (G. 1.2).

(43) MgSO-Magnesium Sulphate.

(44) Albumen.

(45) $\mathrm{PtCl}$-Platinic Chloride.

(46) $\mathrm{NaNO}_{3}-$ Sodium Nitrate.

(47) $\mathrm{HgCl}_{2}-$ Bichloride of Mercury.

(48) $\mathrm{AuCl}_{3}-$ Terchloride of Gold.

(49) $\mathrm{SnO}-$ Stannic Oxide.

(50) $\mathrm{K}_{6} \mathrm{Fe}_{2} \mathrm{Cy}_{12}-$ Potassium Ferricyanide.

The following summary of characteristic qualitative tests are after Prof. A. J. Moses. They are arranged below in the following order:

First-In the dry way or by means of the blowpipe.

Second-In the wet way or by means of wet resgents. 
QUALITATIVE TESTS OF MINERALS.

ALUMINUM, Al.

WITH THE BLOWPIPE.

With Soda.-Swells and forms an infusible compound.

With Borax or S. Ph.-Clear or cloudy, never opaque.

With Cobalt Solution.-Fine blue when cold. (Certain phosphates, borates and fusible silicates become blue in absence of alumina.)

IN THE WET WAY.

1. Alkali hydroxides precipitate grayish-white, $\mathrm{Al}=(\mathrm{HO})^{\circ}$, soluble in fixed alkali-hydroxides, but only slightly soluble in $\mathrm{NH}_{4} \mathrm{OH}$ if $\mathrm{NH}_{4} \mathrm{Cl}$ is present.

2. Basic acetate of aluminium is precipitated by addition of $\mathrm{NaC}_{2} \mathrm{H}_{8} \mathrm{O}_{2}$ to a warm and slightly acid solution.

Confirm.-By blowpipe test.

\section{AMMONIA, $\mathrm{NH}_{3}$.}

WITH THE BLOWPIPE.

In Closed Tube.-Evolution of gas with the characteristic odor. Soda or lime assists the reaction. The gas turns red litmus paper blue, and forms white clouds with $\mathrm{HCl}$ vapor.

\section{ANTIMONY, Sb.}

WITH THE BLOWPIPE.

On Coal, $R . F .-$ Volatile white coat, bluish in thin layers, continues to form after cessation of blast. (This coat may be further tested by $\mathrm{S}$. Ph. or flame.)

With Bismuth Flux:-On Plaster.-Orange-red coat, made orange by $\left(\mathrm{NH}_{4}\right)_{2} \mathrm{~S}$.

On Coal.-Faint yellow or red coat.

In Open Tube.-Dense, white, non-volatile, amorphous sublimate. The sulphide, too rapidly heated, will yield spots of red. 
In Closed Tube.-The oxide will yield a white fusible sublimate of needle crystals; the sulphide, a black sublimate, red when cold.

Flame-Pale yellow-green.

With S. Ph.-Dissolved by O. F., and treated on coal with tin in R. F. becomes gray to black.

\section{Interfering Elements.}

Arsenic.-Remove by gentle 0 . F. on coal.

Arsenic with Sulphur.-Remove by gently heating in closed tube.

Copper.-The S. Ph. bead with tin in R. F. may be momentarily red, but will blacken.

Lead or Bismuth.-Retard formation of their coats by intermittent blast, or by boracic acid. Confirm cost by flame, not by $\mathrm{S}$. $\mathrm{Ph}$.

\section{IN THE WET WAY.}

1. $\mathrm{H}_{2} \mathrm{~S}$ precipitates orange-red $\mathrm{Sb}_{2} \mathrm{~S}_{3}$ from acid solutions. The precipitate is soluble in $\mathrm{HCl}$, in alkalies, and in alkaline sulphides.

To distinguish between $\mathrm{Sb}_{2} \mathrm{O}_{3}$ and $\mathrm{Sb}_{2} \mathrm{O}_{3}$, add solution of $\mathrm{AgNO}_{3}$, in the presence of $\mathrm{KOH}$ or $\mathrm{NaOH}$. $\mathrm{Sb}_{3} \mathrm{O}_{3}$ precipitates black, $\mathrm{Ag} 4 \mathrm{O}$, which is insoluble in $\mathrm{NH}, \mathrm{OH}$; and $\mathrm{Sb}_{2} \mathrm{O}_{5}$ precipitates white, $\mathrm{AgSbO}_{3}$, which is soluble in $\mathrm{NH} \cdot \mathrm{OH}$.

ARSENIC, As.

WITH THE BLOWPIPE.

On Smoked Plaster.-White coat of octahedral crystals.

On Coal.-Very volatile white coat and strong garlic odor. The oxide and sulphide should be mixed with soda.

With Bismuth Flux:-On Plaster--Reddisborange coat, made yellow by (NH،) $2 \mathrm{~S}$.

On Coal.-Faint-yellow coat. 
In Open Tube.-White sublimate of octahedral crystals. Too high heat may form brown suboxide or red or yellow sulphide.

In Closed Tube.-May obtain white oxide, yellow or red sulphide, or black mirror of metal.

Flame.-Pale azure-blue.

\section{Interfering Elements.}

Antimony.-Heat in closed tube with soda and charcoal, treat resulting mirror in 0 . F. for odor.

Cobalt or Nickel.-Fuse in 0 . F. with lead and recognize by odor.

Sulphur.-(a) Red to yellow sublimate of sulphide of arsenic in closed tube.

(b) Odor when fused with soda on coal.

IN THE WET WAY.

1. $\mathrm{H}_{2} \mathrm{~S}$ precipitates yellow $\mathrm{As}_{2} \mathrm{~S}_{3}$ best from $\mathrm{HCl}$ solutions. Soluble in alkalies and alkaline sulphides, insoluble in $\mathrm{HCl}$.

2. $\mathrm{H}_{2} \mathrm{~S}$ precipitates yellow, $\mathrm{As}_{2} \mathrm{~S}$ s from acid solutions after heating solution and passing gas for some time.

3. $\mathrm{AgNO}_{3}$ precipitates yellow $\mathrm{Ag}_{3} \mathrm{AsO}_{3}$ or reddishbrown $\mathrm{Ag}_{s} \mathrm{AsO}_{\mathrm{s}}$, soluble in dilute acids, ammonia, and ammonia salts.

4. CuSO, precipitates yellowish-green $\mathrm{Cu}_{3}\left(\mathrm{AsO}_{s}\right)^{-}$, or greenish-blue, $\mathrm{CuHAsO}$, soluble in $\mathrm{NH} . \mathrm{OH}$ and $\mathrm{NH}_{4} \mathrm{Cl}$.

5. Ammonium magnesia mixture precipitates white $\mathrm{MgNH} \cdot \mathrm{AsO}$.

BARIUM, Ba.

WITH THE BLOWPIPE.

On Coal, with Soda.-Fuses and sinks into the coal. Flame.-Yellowish green, improved by moistening with $\mathrm{HCl}$. 
With Borax or S. Ph.-Clear and colorless; can be flamed opaque white.

IN THE WET WAY.

1. Alkali carbonates precipitate white $\mathrm{BaCO}_{3}$ soluble in $\mathrm{HCl}$ and $\mathrm{HNO}_{3}$. Soluble in acids.

2. Soluble sulphates and $\mathrm{H}_{2} \mathrm{SO}$, precipitate white $\mathrm{BaSO}_{4}$, which is practically insoluble in acids and water.

Confirm.-By blowpipe test.

\section{BISMU'TH, Bi.}

WITH THE BLOWPIPE.

On Coal.-In either flame is reduced to brittle metal and yields a volatile coat, dark orange-yellow hot, lemon-yellow cold, with yellowish-white border.

With Bismuth Flux (sulphur, 2 parts; potassic iodide, 1 part; potassic bisulphate, 1 part) :-On Plaster.-Bright-scarlet coat surrounded by chocolatebrown with sometimes a reddish border. The brown may be made red by ammonia. (May be obtained by heating $\mathrm{S}$. $\mathrm{Ph}$. on the assay.)

On Coal.-Bright-red coat with sometimes an inner fringe of yellow.

With S. Ph.-Dissolved by O. F. and treated on coal with tin in R. F. is colorless hot, but blackish gray and opaque cold.

\section{Interfering Elements.}

Antimony.-Treat on coal with boracic acid, and treat the resulting slag on plaster with bismuth flux.

Lead.-Dissolve coat in S. Ph., as above.

IN THE WET WAY.

1. $\mathrm{H}_{2} \mathrm{~S}$ or $\left(\mathrm{NH}_{4}\right)_{2} \mathrm{~S}$ precipitates brownish-black $\mathrm{Bi}_{2} \mathrm{~S}_{3}$ insoluble in dilute acids, but soluble in strong HNOs.

2. $\mathrm{H}_{2} \mathrm{O}$ precipitates from the chloride white $\mathrm{BiO}$ 
Cl, insoluble in an excess, but soluble in $\mathrm{HCl}$ and HNOs.

3. $\mathrm{SnCl}$ in the presence of $\mathrm{NaOH}$ or $\mathrm{KOH}$ precipitates black $\mathrm{Bi}_{2} \mathrm{O}$.

Confirm.-By blowpipe test.

BROMINE, Br.

WITH THE BLOWPIPE.

With S. Ph., saturated with CuO.-Treated at tip of blue flame, the bead will be surrounded by greenishblue flame.

In Matrass with $\mathrm{KHSO}_{4}$-Brown, choking vapor. Interfering Elements.

Silver.-The bromine melts in KHSO and forms a blood-red globule, which cools yellow and becomes green in the sunlight.

IN THE WET WAY.

1. $\mathrm{AgNO}$ precipitates yellow-white $\mathrm{AgBr}$; changes to gray, soluble in $\mathrm{KCN}$, slightly soluble in $\mathrm{NH}_{4} \mathrm{OH}$, insoluble in $\mathrm{HNO}_{3}$.

Separation of $\mathrm{Cl}, \mathrm{Br}$, and I.-Place a solution of the mixture in a test-tube with a little $\mathrm{MnO}_{2}$ and water, add a drop of dilute $\mathrm{H}_{2} \mathrm{SO}_{4}$ (one in ten). A brown color indicates I. Boil; violet vapors are given off. When these cease add 2cc. of $\mathrm{H}_{2} \mathrm{SO}_{4}$ and boil; brown vapors indicate $\mathrm{Br}$. Boil until brown vapors cease and cool. When cold, add an equal volume of $\mathrm{H}_{2} \mathrm{SO}_{4}$ and heat; green vapors indicate $\mathrm{Cl}$.

\section{BORON, B.}

WITH THE BLOWPIPE.

All borates intumesce and fuse to a bead.

Flame.-Yellowish green. May be assisted by: (a) Moistening with $\mathrm{H}_{2} \mathrm{SO}_{4}$; (b) Mixing to paste with water, and boracic-acid flux (41/2 parts $\mathrm{KHSO}_{4}, 1$ part $\mathrm{CaF}_{2}$ ); (c) By mixing to paste with $\mathrm{H}_{2} \mathrm{SO}_{4}$ and $\mathrm{NH} \cdot \mathrm{F}$. 
IN THE WET WAY.

1. $\mathrm{BaCl}_{2}$ and $\mathrm{CaCl}_{2}$ precipitate white $\mathrm{Ba}_{3}(\mathrm{BO})$ : and $\mathrm{Ca}_{3}\left(\mathrm{BO}_{3}\right)_{2}$.

2. $\mathrm{AgNO}_{3}$ precipitates white $\mathrm{Ag}_{3} \mathrm{BO}_{3}$ 。

3. Free boracic acid turns turmeric paper brownish red, becoming more intense when the paper is dried. When mixed with $\mathrm{HCl}$ to acid reaction and dried it becomes red.

CADMIUM, Cd.

WITH THE BLOWPIPE.

On Coal, R. F.-Dark-brown coat, greenish yellow in thin layers. Beyond the coat, at first part of operation, the coal shows a variegated tarnish.

On Smoked Plaster with Bismuth Flux.-White coat made orange by $\left(\mathrm{NH}_{4}\right)_{2} \mathrm{~S}$.

With Borax or S. Ph.-O. F. Clear yellow hot, colorless cold; can be flamed milk-white. The hot bead touched to $\mathrm{Na}_{2} \mathrm{~S}_{2} \mathrm{O}_{3}$ becomes yellow.

R. F. Becomes slowly colorless.

\section{Interfering Elements.}

Lead, Bismuth, Zinc.-Collect the coat, mix with charcoal dust, and heat gently in a closed tube. Cadmium will yield either a reddish-brown ring or a metallic mirror. Before collecting coat treat it with $\mathrm{O}$. F. to remove arsenic.

IN THE WET WAY.

1. $\mathrm{H}_{2} \mathrm{~S}$ or $\left(\mathrm{NH}_{4}\right)_{2} \mathrm{~S}$ precipitates yellow CdS, insoluble in dilute acids, alkalies, alkali sulphides, or cyanides. Soluble in strong hot $\mathrm{HCl}, \mathrm{HNO}_{3}$, and $\mathrm{H}_{2} \mathrm{SO}_{4}$.

2. $\mathrm{Zn}$ precipitates from acid and ammoniacal solutions gray $\mathrm{Cd}$.

3. $\mathrm{KOH}$ and $\mathrm{NaOH}$ precipitate white $\mathrm{Cd}(\mathrm{OH})_{2}$, insoluble in excess; whilst $\mathrm{NH} \cdot \mathrm{OH}$ precipitate white $\mathrm{Cd}(\mathrm{OH})$, which is soluble in excess.

Confirm.-By blowpipe test. 


\section{CALCIUM, Ca.}

WITH THE BLOWPIPE.

On Coal, with Soda.-Insoluble, and not absorbed by the coal.

Flame.-Yellowish red, improved by moistening with $\mathrm{HCl}$.

With Borax or S. Ph.-Clear and colorless, can be flamed opaque.

IN THE WET WAY.

1. $\mathrm{H}_{2} \mathrm{SO}_{4}$ precipitates white $\mathrm{CaSO}_{4}$, soluble in a concentrated solution of ( $\left.\mathrm{NH}_{4}\right)_{2} \mathrm{SO}_{4}$; distinction from $\mathrm{Ba}$ and $\mathrm{Sr}$.

2. Alkaline arseniates precipitate $\mathrm{CaHASO}_{2}$, soluble in acids and $\mathrm{NH}_{4} \mathrm{OH}$. $\mathrm{Ba}, \mathrm{Sr}$, and $\mathrm{Mg}$ give this precipitate only in concentrated solutions. Ammonia salts must be absent.

Confirm.-By plowpipe test.

CARBONIC ACID, $\mathrm{CO}_{2}$.

WITH THE BLOWPIPE.

With Nitric Acid.-Heat with water and then with dilute acid; $\mathrm{CO}_{2}$ will be set free with effervescence. The escaping gas will render lime-water turbid.

With Borax or S. Ph.-After the flux has been fused to a clear bead, the addition of a carbonate will cause effervescence during further fusion.

IN THE WET WAY.

1. Add $\mathrm{HNO}_{3}$ to substance in a test-tube, and pass gas through a solution of lime-water. A white precipitate $\mathrm{CaCO}$ s indicates $\mathrm{CO}_{2}$.

\section{CHLORINE, Cl.}

a WITH THE BLOWPIPE.

With S. Ph., saturated with CuO.-Treated at tip of blue flame the bead will be surrounded by an intense azure-blue flame. 
On Coal, with $\mathrm{CuO}$.-Grind with a drop of $\mathrm{H}_{2} \mathrm{SO}_{4}$, spread the paste on coal, dry gently in 0 . F., and treat with blue flame, which will be colored greenish blue and then azure-blue.

IN THE WET WAY.

1. $\mathrm{AgNO}_{3}$ precipitates white $\mathrm{AgCl}$, soluble in $\mathrm{NH} . \mathrm{OH}$.

\section{CHROMIUM, Cr.}

WITH THE BLOWPIPE.

With Borax or S. Ph.-O. F. Reddish hot, fine yellow green cold.

R. F. In borax, green hot and cold. In S. Ph. red hot, green cold.

With Soda.-O. F. Dark yellow hot, opaque and light yellow cold.

R. F. Opaque and yellowish green cold.

\section{Interfering Elements.}

Manganese.-The soda bead in 0 . F. will be bright yellowish green.

\section{IN THE WET WAY.}

1. $\mathrm{NH}_{4} \mathrm{OH}$ precipitates bluish green $\mathrm{Cr}_{2}(\mathrm{OH})_{\text {, }}$ slightly soluble in excess.

2. From solutions of $\mathrm{CrO}_{3}$ lead salts precipitate yellow $\mathrm{PbCrO}_{4}$, soluble in $\mathrm{HNO}_{3}$ and insoluble in acetic acid. Difficulty soluble in $\mathrm{KOH}$.

3. A very delicate test for $\mathrm{Cr}$ as $\mathrm{CrO}_{3}$ is by means of $\mathrm{H}_{2} \mathrm{O}_{2}$ (hydrogen peroxide) and ether, giving a fine blue color.

\section{COBALT, Co.}

WITH THE BLOWPIPE.

On Coal, $R$. F.-The oxide becomes magnetic metal. The solution in $\mathrm{HCl}$ will be rose-red, but on svaporation will be blue.

With Borax or S. Ph.-Pure blue in either flame. 


\section{Interfering Elements.}

Arsenic.-Roast and scorify with successive additions of borax. There may be, in order given: Yellow (iron), green (iron and cobalt), blue (cobalt), reddish brown (nickel), green (nickel and copper), blue (copper).

Copper and other Elements which Color Strongly. -Fuse with borax and lead in coal in R. F. The borax on platinum wire in 0 . F. will show the cobalt, except when obscured by much iron or chromium.

Iron, Nickel, or Chromium.-Fuse in R. F. with a little metallic arsenic, then treat as an arsenide.

Sulphur or Selenium.-Roast and scorify with borax, as before described.

IN THE WET WAY.

1. Fixed alkalies precipitate blue basic salts. This precipitate absorbs oxygen and becomes olive-green hydroxide. If boiled before oxidation in the air becomes rose-red $\mathrm{Co}(\mathrm{OH})$; does not dissolve in excess. HN. $\mathrm{OH}$ produces the same precipitate, which is soluble in excess.

2. $\mathrm{K}_{3} \mathrm{FeC}_{6} \mathrm{~N}_{6}$ precipitates dark brown $\mathrm{Cos}_{2}(\mathrm{FeC}$ $\mathrm{N}_{\text {o) }}$, insoluble in $\mathrm{HCl}$. If to a solution of $\mathrm{Co}$ or $\mathrm{Ni}$ an excess of $\mathrm{NH}_{4} \mathrm{Cl}$ and $\mathrm{NH}_{4} \mathrm{OH}$ is added and then $\mathrm{K}_{8} \mathrm{Fe}$ $\mathrm{C}_{\odot} \mathrm{N}_{\text {, }}$ a blood-red color indicates $\mathrm{Co}$. If $\mathrm{Ni}$ is present, and the solution is boiled, a copper-red precipitate forms; if any $\mathrm{Co}$ is present, a dirty green, on boiling.

3. To a dilute solution of cobaltous nitrate add tartaric or citric acid, then an excess of ammonia, and a few drops of potassium ferricyanide; a deep-red color appears; even if largely diluted.

Confirm.-By blowpipe test. 
COPPER, Cu.

\section{WITH THE BLOWPIPE.}

On Coal, R. F.-Formation of red metallic metal.

Flame.-Emerald-green or azure-blue, according to compound. The azure-blue flame may be obtained (sulphur, selenium, and arsenic should be removed by roasting; lead necessitates a gentle heat) -

(a) By moistening with $\mathrm{HCl}$ or aqua regia, drying gently in O. F., and heating strongly in R. F. ;

(b) By saturating $\mathrm{S}$. Ph. bead with substance, adding common salt, and treàting with blue flame.

With Borax or S. Ph.-O. F. Green hot, blue or greenish blue cold. (By repeated slow oxidation and reduction, a borax bead becomes ruby-red.)

R. F. Greenish or colorless hot, opaque and brownish red cold. With tin on coal this reaction is more delicate.

\section{Interfering Elements.}

General Method.-Roast thoroughly, treat with borax on coal in strong R. F. (oxides, sulphides, sulphates, are best reduced by a mixture of soda and borax), and-

If Button Forms.- Separate the button from the slag, remove any lead from it by O. F., and make either S. Ph. or flame test upon residual button.

If No Visible Button Forms.-Add test lead to the borax fusion, continue the reduction, separate the button, and treat as in next test (lead alloy).

Lead or Bismuth Alloys.-Treat with frequently changed boracic acid in strong $R$. F., noting the appearance of slag and residual button.

Trace.-A red spot in the slag.

Over One Per Cent.-The residual button will be bluish green; when melted will dissolve in the slag and color it red upon application of the 0 . F., or may be re- 
moved from the slag and be submitted to either the S. $\mathrm{Ph}$. or the flame test.

IN THE WET WAY.

1. $\mathrm{HN}+\mathrm{OH}$ produces a deep-blue solution.

2. $\mathrm{NaOH}$ and $\mathrm{KOH}$ when added to saturation precipitate blue $\mathrm{Cu}(\mathrm{OH})_{2}$, insoluble in excess. When boiled the precipitate changes to black $\mathrm{Cu}_{3} \mathrm{O}_{2}(\mathrm{OH})_{2}$. Organic substances generally prevent the formation of this precipitate.

3. Fe and $\mathrm{Zn}$ precipitate metallic copper from cupric solutions.

\section{FLUORINE, F.}

WITH THE BLOWPIPE.

Etching Test.-If fluorine be released it will corrode glass in cloudy patches, and in presence of silica there will be a deposit on the glass. According to the refractoriness of the compound the fluorine may be released-

(a) In closed tube by heat;

(b) In closed tube by heat and $\mathrm{KHSO}_{4}$;

(c) In open tube by heat and glass of $\mathrm{S} . \mathrm{Ph}$.

With Conc. $\mathrm{H}_{2} \mathrm{SO}_{4}$ and $\mathrm{SiO}_{2}$,-If heated, and the fumes condensed by a drop of water upon a platinum wire, a film of silicic acid will form upon the water.

\section{IODINE, I.}

WITH THE BLOWPIPE.

With S. Ph., saturated with $C u O$.- Treated at the tip of the blue flame, the bead is surrounded by an intense emerald-green flame.

In Matrass with KHSO.-Violet, choking vapor and brown sublimate.

In Open Tube, with equal parts Bismuth Oxide, Sulphur, and Soda.-A brick-red sublimate.

With Starch Paper.-The vapor turns the paper dark purple. 


\section{Interfering Elements.}

Silver.-The iodide melts in KHSO, to a dark-red globule, yellow on cooling, and unchanged by sunlight.

$$
\text { IRON, Fe. }
$$

WITH THE BLOWPIPE.

On Coal.-R. F. Many compounds becume magnetic. Soda assists the reaction.

With Borax.-0. F. Yellow to red hot, colorless to yellow cold. (A slight yellow color can only be attributed to iron when there is no decided color produced by either flame in highly-charged beads of borax and $\mathrm{S}$. $\mathrm{Ph}$.)

R. F. Bottle-green. With tin on coal, violet-green. With S. Ph.-O. F. Yellow to red hot, greenish when cooling. Colorless to yellow cold.

R. F. Red hot and cold, greenish while cooling.

State of the Iron.-A borax-blue bead from $\mathrm{CuO}$ is made red by $\mathrm{FeO}$ and greenish by $\mathrm{Fe}_{2} \mathrm{O}_{3}$.

\section{Interfering Elements.}

Chromium.-Fuse with nitrate and carbonate of soda on platinum, dissolve in water, and test residue for iron.

Cobalt.-By dilution the blue of cobalt in borax may often be lost before the yellow of iron.

Copper.-May be removed from borax bead by fusion with lead on coal in $\mathrm{R}$. F.

Manganese.-(a) May be faded from borax bead by treatment with tin on coal in R. F.;

(b) May be faded from S. Ph. bead by R. F.

Nickel.-May be faded from borax bead by R. F.

Tungsten or Titanium.-The S. Ph. bead in $\mathrm{R}$. F. will be reddish brown instead of blue or violet.

Uranium.-As with chromium. 
Alloys, Sulphides, Arsenides, etc.-Roast, treat with borax on coal in R. F., then treat borax in R. F. to remove reducible metals.

IN THE WET WAY.

FeO.-1. $\mathrm{K}_{3} \mathrm{FeC}_{6} \mathrm{~N}_{6}$ precipitates dark-blue $\mathrm{Fe}_{3}(\mathrm{Fe}$ $\mathrm{C} \cdot \mathrm{N})$ ), insoluble in acids.

2. NH.OH precipitates white $\mathrm{Fe}(\mathrm{OH})$.

$\mathrm{Fe}_{2} \mathrm{O}_{3}$ - - 1. NH.CNS produces a blood-red solution.

3. $\mathrm{NH}_{3} \mathrm{OH}$ precipitates brownish $\mathrm{Fe}(\mathrm{OH})_{0}$ 。

$$
\text { LEAD, } \mathrm{Pb} \text {. }
$$

WITH THE BLOWPIPE.

On Coal.-In either flame is reduced to malleable metal, and yields near the assay a dark lemon-yellow coat, sulphur-yellow cold, and bluish white at border. (The phosphate yields no coal without the aid of a flux.)

With Bismuth Flux:-On Plaster.-Chrome-yellow coat, blackened by $\left(\mathrm{NH}_{4}\right)_{2} \mathrm{~S}$.

On Coal.-Volatile yellow coat, darker hot.

Flame.--Azure-blue.

With Borax or S. Ph.-0. F. Yellow hot, colorless cold. Flames opaque yellow.

R. F. Borax bead becomes clear, S. Ph. bead cloudy.

Interfering Elements.

Antimony.-Treat on coal with boracic acid, and treat the resulting slag on plaster with bismuth flux.

Arsenic Sulphide.-Remove by gentle O. F.

Cadmium.-Remove by R. F.

Bismuth.-Usually the bismuth-flux tests on plaster are sufficient. In addition the lead coat should color the R. F. blue. 
IN THE WET WAY.

1. Zn precipitates crystals of $\mathrm{Pb}$.

2. $\mathrm{H}_{2} \mathrm{SO}$, precipitate white $\mathrm{PbSO}_{4}$, slightly soluble in excess, insoluble in alcohol, but soluble in ammonium acetate or citrate.

3. $\mathrm{H}_{2} \mathrm{~S}$ or $\left(\mathrm{NH}_{4}\right)_{2} \mathrm{~S}$ precipitates black $\mathrm{PbS}$, soluble in $\mathrm{HNO}_{3}$ with formation of $\mathrm{PbSO}_{4}$.

4. $\mathrm{K} \mathrm{FeC}_{6} \mathrm{~N}_{6}$ precipitates white $\mathrm{Pb}_{2} \mathrm{FeC}_{8} \mathrm{~N}_{\text {。 }}$.

\section{LITHIUM, Li.}

WITH THE BLOWPIPE.

Flame-Crimson, best obtained by gently heating near the wick.

Interfering Elements.

Sodium.-(a) Use a gentle flame and heat near the wick; $(b)$ Fuse on platinum wire with baric chloride in 0 . F. The flame will be first strong yellow, then green, and, lastly, crimson.

Calcium or Strontium.-As these elements do not color the flame in the presence of baric chloride, the above test will answer.

Silicon.-Make into a paste with boracic-acid flux and water, and fuse in the blue flame. Just after the flux fuses the red flame will appear.

IN THE WET WAY.

1. Nitrophenic acid forms a yellow precipitate.

2. $\mathrm{Na}_{2} \mathrm{CO}_{3}$ precipitates white $\mathrm{Li}_{2} \mathrm{CO}_{3}$, slightly soluble in $\mathrm{H}_{2} \mathrm{O}$.

Confirm.-By blowpipe and spectroscope.

\section{MAGNESIUM, Mg.}

WITH THE BLOWPIPE.

On Coal, with Soda.-Insoluble, and not absorbed by the coal.

With Borax or S. Ph.-Clear and colorless; can be flamed opaque-white. 
With Cobalt Solution.-Strongly heated, becomes a pale-flesh color. - (With silicates this action is of use only in the absence of coloring oxides. The phosphate, arsenate, and borate become violet-red.)

IN THE WET WAY.

1. NasHPO، precipitates, in presence of $\mathrm{NH} \cdot \mathrm{OH}$ and $\mathrm{N}_{4} \mathrm{HCl}$, white $\mathrm{MgNH}+\mathrm{PO}$. Fine crystals.

Confirm.-By blowpipe.

MANGANESE, Mn.

WITH THE BLOWPIPE.

With Borax or S. Ph.-O. F. Amethystine hot, reddens on cooling. With much, is black and opaque. (The colors are more intense with borax than with $\mathrm{S}$. $\mathrm{Ph}$.) If a hot bead is touched to a crystal of sodic nitrate an amethystine or rose-colored froth is formed.

R. F. Colorless or with black spots.

With Soda.-O. F. Bluish green and opaque when cold. Sodic nitrate assists the reaction.

Interfering Elements.

Chromium.-The soda bead in $\mathrm{O}$. F. will be bright yellowish green instead of bluish green.

Silicon.-Dissolve in borax, then make soda fusion. IN THE WET WAY.

1. Boil with $\mathrm{HNO}_{3}$, and add peroxide of lead. A reddish-violet solution (color of potassium permanganate) indicates $\mathrm{Mn}$.

\section{MERCURY, Hg.}

WITH THE BLOWPIPE.

With Bismuth Flux:-On Plaster.-Volatile yellow and scarlet coat. If too strongly heated the coat is black and yellow.

On Coal.-Faint-yellow coat at a distance.

In Matrass, with Dry Soda or with Litharge.Mirror-like sublimate, which may be collected in glo- 
bules. (Gold-leaf is whitened by the slightest trace of vapor of mercury.)

IN THE WET WAY.

1. A piece of bright metallic copper is coated with a precipitate of metallic $\mathrm{Hg}$, upon insertion in a solution of $\mathrm{Hg}$.

2. $\mathrm{SnCl}_{2}$ precipitates first white $\mathrm{Hg}_{2} \mathrm{Cl}_{2}$ and then gray $\mathrm{Hg}$.

To distinguish between mercurous and mercuric compounds $\mathrm{HCl}$ precipitates white $\mathrm{Hg}_{2} \mathrm{Cl}_{2}$, soluble in aqua regia, $\mathrm{HNO}_{3}$, and $\mathrm{NH}_{4} \mathrm{Cl}$, and blackened by $\mathrm{NH}_{4}$ $\mathrm{OH}$, from mercurous compounds. No precipitate on addition of $\mathrm{HCl}$ to mercuric compounds.

\section{MOLYBDENUM, Mo.}

\section{WITH THE BLOWPIPE.}

On Coal.-0. F. A coat yellowish hot, white cold; crystalline near assay.

R. F. The coat is turned in part deep blue, in part dark copper-red.

Flame-Yellowish green.

With Borax.-0. F. Yellow hot, colorless cold.

R. F. Brown to black and opaque.

With S. Ph.-O. F. Yellowish green hot, colorless cold. (Crushed between damp unglazed paper becomes red, brown, purple, or blue, according to amount present.)

R. F. Emerald-green.

Dilute (1/4) $\mathrm{HCl}$ Solutions.-If insoluble, the substance may first be fused with $\mathrm{S}$. $\mathrm{Ph}$. in $\mathrm{O}$. F. Then. if dissolved in the acid and heated with metallic tin, zinc, or copper, the solutions will be successively blue, green, and brown. If the $\mathrm{S}$. Ph. bead has been treated in $\mathrm{R}$. F. the solution will become brown. 
IN THE WET WAY.

Upon heating the acid solution with metallic zinc it will turn successively blue, green and brown.

Confirm.-By plowpipe test.

\section{NICKEL, $\mathrm{Ni}$.}

\section{WITH THE BLOWPIPE.}

On Coal, $R$. F.-The oxide becomes magnetic.

With Borax.-0. F. Violet hot, pale reddish brown cold.

R. F. Cloudy, and finally clear and colorless.

With $S$. Ph.-O. F. Red hot, yellow cold.

R. F. Red hot, yellow cold. On coal with tin becomes colorless.

\section{Interfering Elements.}

General Method.-Saturate two or three borax beads with roasted substance, and treat on coal with strong $R$. F. If a visible button results, separate it from the borax and treat with $\mathrm{S}$. $\mathrm{Ph}$. in the $\mathrm{O}$. F., replacing the S. Ph. when a color is obtained. If no visible button results, add either a small gold button or a few grains of test-lead. Continue the reduction, and-

With Gold.-Treat the gold alloy on coal with S. Ph. in strong 0 . F.

With Lead.-Scorify button with boracic acid to small size, complete the removal of lead by 0 . F. on coal, and treat residual button with $\mathrm{S}$. Ph. in $\mathrm{O} . \mathrm{F}$.

Arsenic.-Roast thoroughly, treat with borax in R. F. as long as it shows color, treat residual button with $\mathrm{S}$. Ph. in 0 . F.

Alloys.-Roast and melt with frequently changed borax in R. F., adding a little lead if infusible. When the borax is no longer colored, treat the residual button with $\mathrm{S}$. Ph. in 0 . F. 
IN THE WET WAY.

1. Alkaline carbonates precipitate green basic carbonate $2 \mathrm{NiCO}_{3}, 3 \mathrm{Ni}(\mathrm{OH})_{2}$, soluble in $\left(\mathrm{NH}_{4}\right)_{2} \mathrm{CO}_{3}$ or, in excess of reagent, with blue or greenish-blue color. Again precipitated by $\mathrm{KOH}$ or $\mathrm{NaOH}$ as pale-green $\mathrm{Ni}(\mathrm{OH})_{2}$.

2. $\mathrm{NH}+\mathrm{OH}$ in excess gives blue color.

3. $\mathrm{KCN}$ precipitates pale-green $\mathrm{NiC}_{2} \mathrm{~N}_{2}$, soluble in excess. Upon boiling with $\mathrm{NaClO}$, black $\mathrm{Ni}(\mathrm{OH})_{3}$ is precipitated. Distinction from $\mathrm{Co}$, which gives a dirtywhite precipitate with $\mathrm{KCN}$, soluble in excess, but no precipitate being formed on boiling with $\mathrm{NaClO}$.

NITRIC ACID, $\mathrm{HNO}_{3}$.

WITH THE BLOWPIPE.

In Matrass with KHSO, or in Closed Tube with Litharge.-Brown fumes with characteristic odor. The fumes will turn ferrous-sulphate paper brown.

IN THE WET WAY.

1. To the solution, in a test-tube, add a saturated solution of ferrous sulphate, and then concentrated sulphuric acid (free from $\mathrm{HNO}_{3}$; a brown ring between the $\mathrm{FeSO}_{4}$ and $\mathrm{H}_{2} \mathrm{SO}_{4}$ indicates $\mathrm{HNO}_{3}$ ).

\section{PHOSPHORUS, $P$.}

WITH THE BLOWPIPE.

Flame-Greenish blue, momentary. Improved by conc. $\mathrm{H}_{2} \mathrm{SO}_{4}$.

In Closed Tube with Dry Soda and Magnesium.The soda and substance are mixed in equal parts and dried, and made to cover the magnesium. Upon strongly heating there will be a vivid incandescence, and the resulting mass, crushed and moistened, will yield the odor of phosphuretted hydrogen.

IN THE WET WAY.

Orthophosphates.-1. Magnesia mixture precipitates white MgNH.PO. 
2. $\mathrm{AgNO}_{3}$ precipitates light-yellow $\mathrm{Ag}_{3} \mathrm{PO}$, soluble in $\mathrm{HNO}_{3}$ and $\mathrm{NH} \cdot \mathrm{OH}$.

3. $(\mathrm{NH})_{2} \mathrm{MoO}_{4}+\mathrm{HNO}_{3}$ precipitates yellow ammonium phospho-molybdate; composition variable. The precipitate is soluble in $\mathrm{NH} \cdot \mathrm{OH}$, in excess of phosphoric acid, and is prevented by organic substances, such as tartaric acid.

Pyrophosphate.-1. $\mathrm{MgSO}_{4}$ precipitates white $\mathrm{Mg}_{2} \mathrm{P}_{2} \mathrm{O}_{7}$, soluble in an excess of either solution. $\mathrm{NH}_{4} \mathrm{OH}$ fails to precipitate it from these solutions. On boiling it separates again. By this reaction pyro can be detected in the presence of phosphoric acid.

2. $\left(\mathrm{NH}_{4}\right)_{2} \mathrm{MoO}+\mathrm{HNO}_{3}$ does not give a precipitate until orthophosphate is formed. Most of the pyrophosphates of the heavy metals (Ag an exception) are soluble in alkali pyrophosphates (distinction from orthophosphates). .

3. $\mathrm{AgNO}_{3}$ precipitates white $\mathrm{Ag}_{4} \mathrm{P}_{2} \mathrm{O}_{7}$, soluble in $\mathrm{HNO}_{3}$ and $\mathrm{NH}_{4} \mathrm{OH}$. Addition of an alkali aids the prerinitation.

Metaphosphoric Acid.-1. Magnesia mixture gives no precipitate.

2. $\left(\mathrm{NH}_{4}\right)_{2} \mathrm{MoO}_{4}+\mathrm{HNO}_{3}$ gives no precipitate.

3. $\mathrm{AgNO}$ precipitates white $\mathrm{AgPO}_{3}$, soluble in alkali metaphosphate solutions (distinction from pyrophosphates).

4. Albumen gives a precipitate (distinction from ortho and pyrophosphates).

5. Fusion with $\mathrm{Na}_{2} \mathrm{CO}_{3}$ converts meta and pyro into orthophosphates.

POTASSIUM, K.

WITH THE BLOWPIPE.

Flame.-Violet, except borates and phosphates. 


\section{Interfering Elements.}

Sodium.-(a) The flame through blue glass will be violet or blue;

(b) A bead of borax and a little boracic acid made brown by nickel will become blue on addition of a potassium compound.

Lithium.-The flame through green glass will be bluish green.

\section{IN THEE WET WAY.}

1. PtCl with $\mathrm{HCl}$ precipitates yellow crystalline $(\mathrm{KCl}){ }_{2} \mathrm{PtCl}_{4}$. Evaporate to dryness. The precipitate is not dissolved by alcohol.

Confirm.-By blowpipe and spectroscope.

\section{SELENIUM, Se.}

WITH THE BLOWPIPE.

On Coal, R. F.-Disagreeable horse-radish odor, brown fumes, and a volatile steel-gray coat with a red border.

In Open Tube.-Steel-gray sublimate with red border, sometimes white crystals.

In Closed Tube--Dark-red sublimate and horseradish odor.

\section{Flame-Azure-blue.}

On Coal, with Soda.-Thoroughly fuse in R. F., place on bright silver, moisten, crush, and let stand. The silver will be blackened.

IN THE WET WAY.

1. $\mathrm{H}_{2} \mathrm{~S}$ precipitates yellow sulphide of selenium, soluble in $\left(\mathrm{NH}_{4}\right)_{2} \mathrm{~S}$. Upon heating the precipitate turns reddish yellow.

2. $\mathrm{SnCl}_{3}+\mathrm{HCl}$ produces a red precipitate of $\mathrm{Se}$, which turns gray at a high temperature.

3. Metallic copper, when placed in a warm solution of selenious acid, containing $\mathrm{HCl}$, becomes black; 
if the fluid remains long in contact with the copper, it turns bright red from separation of selenium.

Confirm.-By blowpipe tests.

\section{SILICON, Si.}

WITH THE BLOWPIPE.

On Coal, with Soda.-With its own volume of soda, disolves with effervescence to a clear bead. With more soda the bead is opaque.

With Borax.-Clear and colorless.

With S. Ph.-Insoluble. The test made upon a small fragment will usually show a translucent mass of undissolved matter of the shape of the original fragment.

When not decomposed by S. Ph., dissolve in borax nearly to saturation, add S. Ph., and re-heat for a moment. The bead will become milky or opaque-white.

IN THE WET WAY.

Silicates are determined by the separation of $\mathrm{SiO}_{2}$. Fuse with $\mathrm{Na}_{2} \mathrm{CO}_{3}+\mathrm{NaNO}_{3}$, dissolve in $\mathrm{HCl}$, and evaporate to dryness. Upon evaporation gelatinous silica will separate out. Upon heating and dissolving with $\mathrm{HCl}$ insoluble $\mathrm{SiO}_{2}$ remains behind.

Confirm.-By blowpipe test.

SILVER, Ag. WITH THE BLOWPIPE.

On Coal.-Reduction to malleable white metal.

With Borax or S. Ph.-O. F. Opalescent.

Cupellation.-Fuse on coal with one volume of borax-glass and one to two volumes of test-lead in $R$. F. for about two minutes. Remove button and scorify it in R. F. with fresh borax, then place button on cupel and blow O. F. across it, using as strong blast and as little flame as are consistent with keeping button melted. 
If the litharge is dark, or if the button freezes before brightening, or if it brightens but is not spherical, rescorify it on coal with borax, add more test-lead, and again cupel, until there remains only a white spherical button of silver.

Dissolve button in $\mathrm{HNO}_{3}$ in a glass and if gold is present it will fall to the bottom as a black powder.

IN THE WET WAY.

1. $\mathrm{HCl}$ precipitates white $\mathrm{AgCl}$, insoluble in $\mathrm{HNO}_{3}$, soluble in $\mathrm{NH}_{4} \mathrm{OH}$.

2. $\mathrm{Cu}$ precipitates metallic Ag.

3. KI precipitates yellow AgI, insoluble in $\mathrm{NH}$. $\mathrm{OH}$, soluble in excess of reagent.

Confirm.-By blowpipe test.

SODIUM, Na.

WITH THE BLOWPIPE.

Flame.-Reddish yellow.

IN THE WET WAY.

1. $(\mathrm{NaCl})_{2} \mathrm{PtCl}_{*}$ crystallizes from its concentrated solutions in red prisms.

2. $\mathrm{KSbO}_{3}$ (in neutral or alkaline solutions) precipitates white $\mathrm{NaSbO}_{3}$. The reagent should be dissolved as wanted, as it is unstable in solution.

Confirm.-By blowpipe and spectroscope.

\section{STRONTIUM, Sr.}

WITH THE BLOWPIPE.

On Coal, with Soda.-Insoluble, absorbed by the coal.

Flame.-Intense crimson, improved by moistening with $\mathrm{HCl}$.

With Borax or S. Ph.-Clear and colorless; can be flamed opaque.

\section{Interfering Elements.}

Barium.-The red flame may show upon first introduction of the sample into the flame, but it is afterwards turned brownish yellow. 
Lithium.-Fuse with baric chloride, by which the lithium flame is unchanged.

IN THE WET WAY.

1. $\mathrm{NaOH}, \mathrm{NH}+\mathrm{OH}, \mathrm{Na}_{2} \mathrm{CO}_{3},\left(\mathrm{NH}_{4}\right)_{2} \mathrm{CO}_{3}$, and $\mathrm{Na}$ s HPO، form precipitates which closely resemble those produced by these reagents with $\mathrm{Ba}$ salts.

Confirm.-By blowpipe tests.

\section{SULPHUR, S. \\ WITH THE BLOWPIPE.}

On Coal, with Soda and a little Borax.-Thoroughly fuse in the R. F. flame, and either,

(a) Place on bright silver, moisten, crush, and let stand. The silver will become brown or black. Or,

(b) Heat with dilute $\mathrm{HCl}$ (sometimes with powdered zinc); the odor of $\mathrm{H}_{2} \mathrm{~S}$ will be observed.

In Open Tube.-Suffocating fumes. Some sulphates are unaffected.

In Closed Tube.-May have sublimate red when hot, yellow cold, or sublimate of undecomposed sulphide, or the substance may be unaffected.

With Soda and Silica (equal parts).-A yellow or red bead.

To Determine whether Sulphide or Sulphate.Fuse with soda on platinum foil. The sulphide only will stain silver.

IN THE WET WAY.

1. $\mathrm{BaCl}_{2}$ gives a white precipitate, $\mathrm{BaSO}$, when added to sulphuric-acid solutions. Practically insoluble.

2. On addition of $\mathrm{HNO}_{3}$ to sulphides $\mathrm{H}_{2} \mathrm{~S}$ is given off.

\section{TELLURIUM, Te.}

WITH THE BLOWPIPE.

On Coal.-Volatile white coat with red or yellow border. If the fumes are caught on porcelain, the re- 
sulting gray or brown film may be turned crimson when moistened with conc. $\mathrm{H}_{2} \mathrm{SO}_{4}$, and gently heated.

On Coal with Soda.-Thoroughly fuse in R. F. Place on bright silver, moisten, crush, and let stand. The silver will be blackened.

Flame-Green.

In Open Tube.-Gray sublimate fusible to clear drops.

With $\mathrm{H}_{2} \mathrm{SO}_{4}$ (conc.).-Boiled a moment, there results a purple-violet solution, which loses color on further heating or on dilution.

IN THE WET WAY.

1. $\mathrm{H}_{2} \mathrm{~S}$ precipitates brown $\mathrm{TeS}_{2}$ from acid solutions. Soluble in $\left(\mathrm{NH}_{4}\right)_{2} \mathrm{~S}$.

2. Boiled with concentrated $\mathrm{H}_{2} \mathrm{SO}$ there results a purple-violet solution, which fades upon further heating or dilution.

Confirm.-By blowpipe tests.

TIN, Sn.

WITH THE BLOWPIPE.

On Coal.-0. F. The oxide becomes yellow and luminous.

R. F. A slight coat, assisted by additions of sulphur or soda.

With Cobalt Solution.-Moisten the coal in front of the assay, with the solution, and blow a strong R. F. upon the assay. The coat will be bluish green when cold.

With CuO in Borax Bead.-A faint-blue bead is made reddish brown or ruby-red by heating a moment in R. F. with a tin compound.

\section{Interfering Elements.}

Lead or Bismuth Alloys.-It is fair proof of tin if such an alloy oxidizes rapidly with sprouting and cannot be kept fused. 
Zinc.-On coal with soda, borax, and charcoal in R. F., the tin will be reduced, the zinc volatilized; the tin may then be washed from the fused mass.

IN THE WET WAY.

Stannous Oxide $(\mathrm{SnO}),-1 . \mathrm{H}_{2} \mathrm{~S}$ precipitates darkbrown $\mathrm{SnS}$, soluble in $\mathrm{HCl}$, in alkalies; moderately soluble in yellow $\left(\mathrm{NH}_{4}\right)_{2} \mathrm{~S}$.

2. $\mathrm{HgCl}_{2}$ precipitates white $\mathrm{Hg}_{2} \mathrm{Cl}_{2}$, with excess black $\mathrm{Hg}$ (distinction from stannic compounds).

3. $\mathrm{AuCl}_{3}$ with free $\mathrm{HCl}$ or $\mathrm{HNO}_{3}$, a purple precipitate.

4. Zn precipitates spongy $\mathrm{Sn}$.

Stannic oxide $\left(\mathrm{SnO}_{2}\right)$.

1. $\mathrm{H}_{2} \mathrm{~S}$ precipitates yellow $\mathrm{SnS}_{2}$, soluble in $\mathrm{HCl}$, in alkalies and alkaline sulphides.

2. $\mathrm{HgCl}_{2}$ no precipitate.

3. $\mathrm{AuCl}_{8}$ no precipitate.

4. Zn precipitates spongy $\mathrm{Sn}$.

Confirm.-By blowpipe tests.

\section{TITANIUM, Ti.}

\section{WITH THE BLOWPIPE.}

With Borax.-0. F. Colorless to yellow hot; colorless cold, opalescent or opaque white by flaming.

R. F. Yellow to brown, enamel-blue by flaming.

With Ph. S.-O. F. as with borax.

R. F. yellow hot, violet cold.

$\mathrm{HCl}$ Solutions.-If soluble, the substance may first be fused with $\mathrm{S}$. $\mathrm{Ph}$. or with soda, and reduced. If then dissolved in dilute acid and heated with metallic tin, the solution will become violet after standing. Usually there will also be a turbid violet precipitate, which becomes white. 


\section{Interfering Elements.}

Iron.-The S. Ph. bead in R. F. is yellow hot, brownish-red cold.

IN THE WET WAY.

1. NH.OH gives a bulky white precipitate, Ti $(\mathrm{OH})$, insoluble in excess.

2. Sn or $\mathrm{Zn}$ boiled in acid solutions after some time give pale-violet or blue solutions, subsequently a blue precipitate, which gradually becomes white.

Confirm.-By blowpipe.

\section{TUNGSTEN, W.}

WITH THE BIOWPIPE.

With Borax.-0. F. Flame colorless to yellow hot, colorless cold; can be flamed opaque white.

R. F. Colorless to yellow hot, yellowish brown cold.

With S. Ph.-0. F. Clear and colorless.

R. F. Greenish hot, blue cold. On long blowing or with tin on coal becomes dark green.

With Dilute $\mathrm{HCl}$.-If insoluble, the substance may first be fused with $\mathrm{S}$. Ph. The solution heated with tin becomes dark blue; with zinc it becomes purple and then reddish brown.

\section{Interfering Elements.}

Iron.-The S. Ph. in R. F. is yellow hot, blood-red cold.

IN THE WET WAY.

1. $\mathrm{SnCl}_{2}$ produces a yellow precipitate on acidifying with $\mathrm{HCl}$, and applying heat the precipitate acquires a beautiful blue color.

2. Heated with $\mathrm{HCl}$ and $\mathrm{Zn}$ the solution becomes purple, and then reddish brown.

3. $\mathrm{K}_{4} \mathrm{FeC} \cdot \mathrm{N}_{\odot}+\mathrm{HCl}$ gives a deep brownish-red color; after some time " precipitate of the same color is produced. 
URANIUM, U.

WITH THE BLOWPIPE.

With Borax.-0. F. Yellow hot, colorless cold; can be flamed enamel-yellow.

R. F. Bottle-green; can be flamed black, but not enamelled.

With S. Ph.-0. F. Yellow hot, yellowish green cold.

R. F. Emerald-green.

Interfering Elements.

Iron.-With S. Ph. in R. F. is green hot, red cold. IN THE WET WAT.

1. $\mathrm{NH}_{4} \mathrm{OH}, \mathrm{KOH}$, and $\mathrm{NaOH}$ produce a yellow precipitate of uranic hydroxide and alkali.

2. $\mathrm{K}_{4} \mathrm{FeC} \mathrm{N}_{\bullet}$ produces a reddish-brown precipitate.

Confirm.-By blowpipe test.

\section{VANADIUM, V.}

WITH THE BLOWPIPE.

With Borax.-0. F. Colorless or yellow hot, greenish-yellow cold.

R. F. Brownish hot, emerald-green cold.

With S. Ph.-0. F. Dark yellow hot, light yellow cold.

R. F. Brownish hot, emerald-green cold.

IN THE WET WAY.

1. $\mathrm{K}_{\triangleleft} \mathrm{FeC} \mathrm{C}_{\bullet}$ produces a green flocculent precipitate, insoluble in acids.

2. Dissolved in $\mathrm{H}_{2} \mathrm{SO}$, and $\mathrm{Zn}$ added the solution becomes successively green, blue, bluish violet, and lavender. 
3. An acidified solution of vanadates upon being shaken with hydrogen dioxide acquires a red tint; if ether is then added, and the solution shaken, its retains its color, the ether remaining colorless.

\section{ZINC, Zn.}

WITH THE BLOWPIPE.

On Coal.-0. F. The oxide becomes yellow and luminous.

R. F. Yellow coat, white when cold, assisted by soda and a little borax.

With Cobalt Solution.-Moisten the coal in front of the assay, with the solution, and blow a strong R. F. upon the assay. The coat will be bright yellow-green when cold.

\section{Interfering Elements.}

Antimony.-Remove by strong O. F., or by heating with sulphur in closed tube.

Cadmium, Lead, or Bismuth.-The combined coats will not prevent the cobalt-solution test.

Tin.-The coats heated in an open tube, with charcoal dust by the O. F., may yield white sublimate of zinc.

IN THE WET WAY.

1. Alkali hydroxides precipitate white $\mathrm{Zn}(\mathrm{OH})^{2}$, soluble in excess of precipitant.

2. $\mathrm{H}_{2} \mathrm{~S}$ precipitates (from neutral or acetic acid solution) white $\mathrm{ZnS}$.

3. $\mathrm{K}_{4} \mathrm{FeC}_{5} \mathrm{~N}_{6}$ percipitates white $\mathrm{Zn}_{2} \mathrm{FeC}_{\odot} \mathrm{N}_{6}$, insoluble in very dilute solutions of $\mathrm{HCl}$.

4. $\left(\mathrm{NH}_{6}\right)_{2} \mathrm{~S}$ precipitates white $\mathrm{ZnS}$, insoluble in $\mathrm{KOH}$ and $\mathrm{HC}_{2} \mathrm{H}_{3} \mathrm{O}_{3}$,

Confirm.-By blowpipe test. 



\section{PART III.}

\section{Determinative Mineralogy}





\section{PART III.}

\section{DETERMINATIVE MINERALOGY.}

The Determination of Minerals by Their Physical Properties With the Aid of Tables.

Physical Properties of Minerals.-To make an offhand determination of the more common minerals met with is an attainment easily acquired; and it is an art in which the intelligent prospector, miner, and mining man should become expert.

While in nature there are minerals so nearly identical in their physical characteristics as to require even chemical analysis in order to distinguish them, in the majority of cases, however, it will be found, that on close examination, marked differences may be discerned and of sufficient prominence, to at once distinguish any one species from any other to which it may at first bear a striking resemblance.

Minerals differ.-(1) In their action before the blowpipe (B. B.). (2) They differ in Lustre. Color. (4) Hardness (H.). (5) Streak. (6) Fracture and cleavage. (7) Tenacity. (8) Crystalline system (Crys. Syst.). (9) Fusibility (Fusib.). (10) In Specific Gravity (G). These distinguishing characteristics of minerals are described below in the order in which they appear in the Analytical Tables of minerals (Page 95). These tables afford all necessary data (when taken in connection with the blowpipe tests, page 46) for the accurate determination of some three hundred 
and forty-five mineral species. In order that the young student may make rapid progress in the study of the science of mineralogy, the following suggestions are offered for his benefit: In the first place he should secure a good working collection of well selected minerals, accurately determined and labeled. ( For which see page 182). With a working collection of 100 or more specimens the student should next familiarize himself with the use of the blowpipe, and to this end he should reproduce or verify as many as possible (or ail) of the blowpipe tests given on pages 54 to 81 . The blowpipe outfits described on page 46 and page 178 are especially recommended for his use. Mineral specimens illustrating the scales of lustre, color, hardness, fusibility, crystallization, etc., (as described below and given on page 178), should by all means form a part of his outfit.

We will now proceed with a description of the physical properties of minerals, and after fully describing the nature of these properties, we will arrange them in tables with the names of the mineral species in accordance with the degree of which the respective species partake of these properties.

\section{FIRST PROPERTY.} COMPOSITION.

Minerals differ in their chemical composition, and in extreme cases even quantitative chemical analysis must be resorted to in order to distinguish them. The qualitative blowpipe tests (page 54 ) will in general be found fully sufficient to indicate the chemical composition of mineral near enough for all practical purposes of identification. It should here be borne in mind that minerals are of definite chemical composition, while ores, like rocks (page 149), may consist of two or more minerals. In testing a mineral, 
therefore, the single mineral should be taken, not a mixture of mineral, otherwise the analysis would be misleading.

Where the complete chemical analysis of the mineral species are not given in the tables, those elements forming their composition are stated in all cases.

\section{SECOND PROPERTY.}

\section{LUSTRE.}

The lustre of minerals depends on that property possessed by some to reflect light from their surface. The kinds of lustre are:

(a) Metallic. The usual lustre of metals.

(b) Vitreous.-The lustre of broken glass.

(c) Resinous.-The lustre of the yellow resins.

(d) Pearly.-Like pearl, talc, etc.

(e) Greasy.-Looking as if smeared with oil.

(f) Silky.-Like silk, a fibrous structure.

(g) Adamantine.-The lustre of the diamond.

(h) Splendent.-Reflecting light with great brilliancy and giving well defined images.

(i) Shining.-The image produced is not well defined.

(j) Dull.-A total absence of lustre, earthy.

Minerals are transparent when the outlines of objects viewed through them are distinct; subtransparent when objects are seen but their outlines are indistinct; translucent when light is transmitted, but objects are not seen; subtranslucent when merely the edges transmit light faintly, and opaque when no light is transmitted at all.

(J.D.Dana.)

THIRD AND FOURTH PROPERTIES. COLOR AND STREAK.

In distinguishing minerals both the external color and the color of a surface that has been 
abraded or scratched with a knife or file are observed. The latter is called the Streak and the powder abraded the Streak Powder. The colors are either metallic or unmetallic. The metallic are named after some familiar metal, as copper red, bronze yellow, brass yellow, steel gray, lead gray, etc.

The unmetallic colors used in characterizing minerals are various shades of white, gray, black, blue. green, yellow, red and brown.

\section{FIFTH PROPERTY \\ HARDNESS (WRITTEN H).}

To ascertain the comparative hardness of a mineral, it is only necessary to draw a file across the specimen or to make trials of scratching one with another. As standards of comparison the following minerals have been selected, increasing gradually in hardness from talc, which is very soft, to the diamond, the hardest substance known. This table, called the scale of hardness, is as follows:

Talc $=1$, Rock salt $=2$, Calcite $=3$, Flourite $=4$, Apatite $=5$, Orthoclase $=6$, Quartz $=7$, Topaz $=8$, Sapphire $=9$, Diamond $=10$.

\section{SIXTH PROPERTY.}

\section{FRACTURE AND CLEAVAGE.}

If a mineral has a poor cleavage and separates or breaks almost as readily in one direction as in another, smooth, curved surfaces often result. This kind of fracture is called conchoidal. Fracture is said to be uneven if on breaking rough, irregular surfaces are exhibited, hackly when a jagged, irregular surface like that of broken cast iron results and splintery when the mineral breaks in splinters or needles. 
Cleavage is that property possessed by most minerals to part or break in certain directions. The direction of cleavage is always parallel to the faces of the geometrical figure to which the mineral tends to assume, and generally will indicate its crystalline system.

In the $\mathrm{I}$, or isometric system cleavage is generally cubic, octahedral and dodechedral. In the VI, or hexagonal system the cleavage is said to be basal when the cleavage is parallel to the base of the prism, and prismatic when parallel to the sides. If the cleavage is equal in three directions, but not at right angles to one another, the mineral belongs to the $R$, or rhombic form of crystalization, and is designated as rhombohedral. In the remaining systems the term basal expresses cleavage as taking place at right angles to the vertical axis of the crystal. Cleavage is called pinacoidal when it is in one direction parallel to the vertical pinacoids of the III, IV, or V systems. In the II system, if the cleavage is in two directions parallel to the sides of a prism it is called prismatic, or in general, the term prismatic means that the cleavage is easiest parallel to the long sides of the crystal of which the mineral partakes. (See Determinative Mineralogy and Blowpipe Analysis by Brush and Penfield, Pages 155 to 225.)

\section{SEVENTH PROPERTY.}

TENACITY.

The following terms are used in denoting those characteristics possessed by some minerals, such as appear in the columns of the tables under the headings of Tenacity:

(a) Brittle.-When a mineral breaks easily, or when parts of the mineral separates in powder on attempting to cut it. 
(b) Malleable.-When slices may be cut off, and these slices will flatten out under the hammer.

(c) Sectile.-When thin slices may be cut off with a knife. All malleable minerals are sectile.

(d) Flexible.-When minerals will bend and remain bent after the bending force is removed.

(e) Elastic.-When after being bent it will spring back to its original position.

\section{EIGHTH PROPERTY.}

\section{CRYSTALLINE SYSTEM.}

Six systems of crystallization are recognizable among minerals, and these occur under an indefinite number of geometrical forms, but the system to which the crystal belongs is indicated by its mathematical symmetry, and in any case will be found to belong to one of the following six fundamental systems:

I. ISOMETRIC, or the cube, in which the three axes are rectangular in intersection, and equal.

II. TETRAGONAL, or the right prism with square bases, in which the three axes are rectangular in intersection; the two lateral axes equal, and unequal to the vertical.

III. овтновномвіс, or the right rectangular prism, including also the right prism with equilateral rhombic bases, in which the three axes are rectangular in intersection, and unequal.

IV. MONOCLINIC, or the oblique prism with rectangular bases, in which there are only one oblique inclination out of the three made by the intersecting axes, and the three axes are unequal.

V. TRICLINIC, or the oblique prism with rhombic bases, in which all the axes are obliquely inclined to one another, and unequal. 
VI. HEXAGONAL, or the six-sided prism (including also the rhombrohedral section $(\mathrm{R})$, or the three-sided pyramid), in which the vertical axes are at right angles to the lateral; the lateral three in number, and intersecting at angles of 60 degrees. (See Dana's Manual of Mineralogy, under System of Crystallization.)

\section{NINTH PROPERTY.}

FUSIBILITY.

The following is the scale of fusibility which has been adapted, beginning with the most fusible mineral, stibnite, it increases to bronzite, a mineral scarcely fusible at all; the scale is, for:

Stibnite $=1 .-$ Fusible in large pieces in the candle flame.

Natrolite=2.-Fusible in small splinters in the candle flame.

Red Garnet $=3$.-Fusible in large pieces with ease in the blowpipe flame.

Actinolite $=4$. - Fusible in large pieces with difficulty in the blowpipe flame.

Orthoclase $=5 .-$ Fusible in small splinters with difficulty in the blowpipe flame.

Bronzite $=6$. - In the blowpipe flame, scarcely fusible at all.

\section{TENTH PROPERTY. \\ SPECIFIC GRAVITY $(G)$.}

The specific gravity of a mineral is its weight compared with that of distilled water, at $60^{\circ} \mathrm{F}$. If a mineral weighs twice as much as a volume of water equal to itself, its specific gravity is 2 ; if three times, it is 3 , and so on. The most practicable way of determining specific gravities is as follows: Take a light glass bottle (specific gravity bottle) and balance it on the scales 
Now fill to the brim with water and balance again; the amount of weight added is equal to the weight of water in the bottle; note this weight, $=\mathbf{A}$. Now pour out a few drops of water, and weigh again, noting the weight as before, = B. Next add the coarsely powdered mineral until the water is again to the brim, and note the weight, $=\mathrm{C}$. The first weight diminished by the second is equal to the weight of water poured out, $=(A-B)$, and the third weight diminished by the second, is equal to the weight of the mineral, $=(\mathrm{C}-\mathrm{B})$; therefore, $(\mathrm{C}-\mathrm{B}) \div(\mathrm{A}-\mathrm{B}=$ the specific gravity sought. An example will best illustrate this: Suppose, after balancing the empty bottle on the scales as described, then filled to the brim with water, it is found that on adding to the other end of the scales $22.523 \mathrm{gms}$. the scales are balanced.

Then weight of water in bottle........ $=25.523 \mathrm{gms}-\mathrm{A}$

Wt. of water after pouring out a few drops $=20.529$ " $=\mathrm{B}$

Therefore, the weight of water poured out $=\overline{1.964}$ " $=\mathrm{A}-\mathbf{B}$

After flling to the brim with ore, weight $=28.808$ " $=\mathrm{C}$

Subtract weight of water after pouring

out a few drops, or ............. $=20.558 "=B$

Then the weight of ore in flask......... $=\overline{5.249}$ " $=\mathrm{C}-\mathrm{B}$

Now since the wt. of ore (5.249) divided by the $w t$. of its equal volume of water (1.964) is the specific gravity of the ore, we have: $5.249 \div 1.964=2.672=\mathrm{G}$. $=$ $[(\mathrm{C}-\mathrm{B}) \div(\mathrm{A}-\mathrm{B})]$. Therefore, 2.672 is the specific gravity sought.

Another method of determining specific gravities is to suspend the lump of mineral from a silk thread; weighing first in the air. This wt. is called A. The mineral is then suspended and weighed emersed in water. This wt. is called $\mathrm{B}$, and $(\mathrm{A}-\mathrm{B})$ is called $\mathrm{C}$. Then $\mathrm{A} \div \mathrm{C}=\mathrm{G},=$ the specific gravity sought. 


\section{INTRODUCTION TO THE ANYLATICAL TA- BLES OF MINERALS.}

The names of minerals as they appear in the following tables are grouped according to the most prominent metal, or element, entering into their composition. Brief descriptions of each mineral as given in the tables are of typical specimens and they are arranged in the following order: In the first column is found the names of the minerals; in the second column, their chemical composition (deduced from formulæ or analyses); in the third, lustre, etc.

Determination of Minerals By the Tables.-To do this test the specimen to be determined very carefully, and ascertain its physical properties; form some idea of the group to which it belongs (i. e., whether a lead mineral, silver mineral, copper mineral, etc.), and note on a slip of paper the following: First, the Group; second, note its Lustre; third, its Color; fourth, Hardness $(H)$; fifth, Streak; sixth, break and note its Fratcture and Cleavage; seventh, note (when breaking) its Tenacity; eighth, if not otherwise recognizable, examine with a magnifying glass, and note its Crystalline System (Crys. Syst.); ninth, note its Fusibility by holding a small splinter of the mineral with one end held between the points of the platinum forceps and the other in the reducing flame (R. F.) of the blowpipe; note the degree by which its edges become rounded and by which the splinter is consumed, or 
not affected at all, and tenth, Specific Gravity $(G)$; this may become necessary in order to render the comparison decisive.

Having formed some idea of the group to which the of physical properties, and turn to the Tables, and specimen belongs, and completed the foregoing tests; take the slip of paper containing the written summary search (under those groups to which the mineral is thought to belong) for a description which will nearest compare with that of the specimen.

If the evidence found in the table is sufficient to identify the specimen, call it by the name given to that mineral appearing in the first left hand column opposite its description.

All questions of doubt arising from approximate determination of mineral species by the tables should be settled by means of the blowpipe, or by some other reliable method of chemical analysis. (See Qualitative Tests, pages 54-81.) 


\section{$D$ issolve vaseline in $4 N_{3}$ 3 or 4 drops - up to lin Amps.}

\section{Analytical Tables of Minerals}

\section{DIVISION I}

\section{MINERALS FORMING ORE}

The ores of the mines may consist of a mixture of two or more minerals, or of a single one. The minerals forming the ores generally occur in a more or less impure state, and hence they are variable in composition-a result due to imperfect crystallization.

In the following division the more common minerals valuable for ores (when occurring in workable quantities), are described. Being able to identify or recognize the kind of minerals present in an ore, an approximate estimate of its value is made possible in any case. 
Gold.

\begin{tabular}{|c|c|c|c|c|c|c|}
\hline$\oplus$ & $\begin{array}{l}\dot{d e p} \\
\dot{g} \theta\end{array}$ & $\begin{array}{l}0 \\
\infty \\
\infty \\
\infty\end{array}$ & 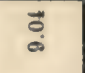 & $\therefore$ & $\begin{array}{l}\text { div } \\
\text { ou }\end{array}$ & 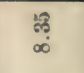 \\
\hline 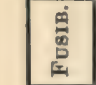 & 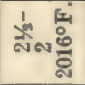 & $=$ & - & $\dddot{m}$ & $\geq^{\infty}$ & 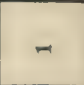 \\
\hline 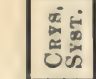 & w & $\triangleright$ & $>$ & 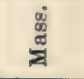 & 浔 & 浔 \\
\hline 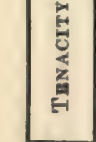 & 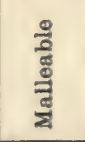 & 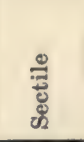 & 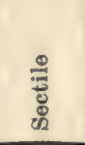 & 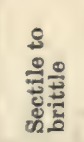 & 苞 & \\
\hline 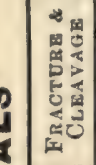 & 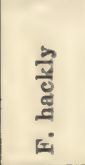 & 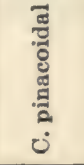 & 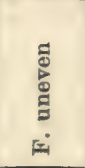 & 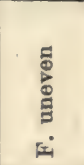 & 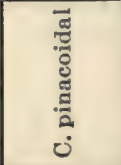 & 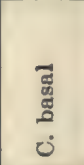 \\
\hline 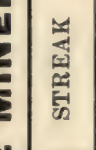 & 跣: & 胥 & 莣 & 胥 & 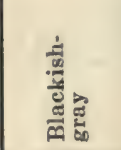 & 莡 \\
\hline$\ddot{H}$ & $\begin{array}{l}x^{*} \\
-\infty \infty\end{array}$ & $\vec{z}_{-\infty}^{\prime}$ & ลे & ลें & $1 \geq 0$ & సे \\
\hline 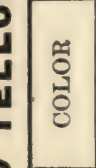 & $\frac{3}{0}$ & 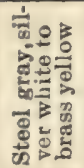 & 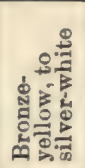 & 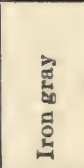 & 蒂 & 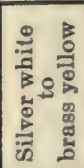 \\
\hline 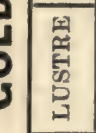 & 葛 & 营 & 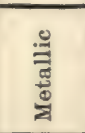 & 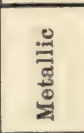 & 总 & 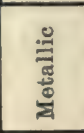 \\
\hline 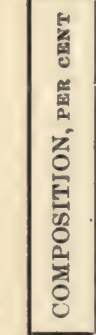 & & 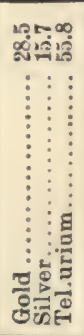 & 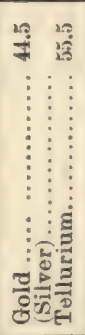 & 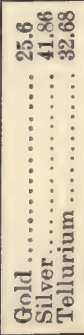 & 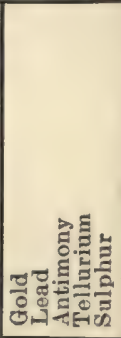 & 흘 \\
\hline 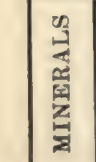 & $\begin{array}{l}\text { 믕 } \\
\frac{0}{0} \\
\frac{0}{E} \\
z\end{array}$ & $\frac{\mathscr{y}}{2}$ & 芯 & : & 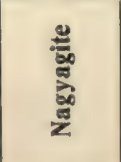 & 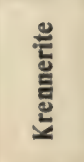 \\
\hline
\end{tabular}


Silver.

\begin{tabular}{|c|c|c|c|c|c|c|c|c|}
\hline & ம் & 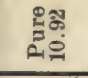 & $\begin{array}{l}1 \\
\neq 10 \\
i-\infty\end{array}$ & $\begin{array}{l}\text { \&్ } \\
\infty \\
\end{array}$ & ลั & $\begin{array}{l}\operatorname{tro} \\
\infty \\
\infty\end{array}$ & $\stackrel{10}{\circ}$ & $\begin{array}{l}10 . \\
\text { मू. }\end{array}$ \\
\hline & 葛 & 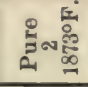 & $\stackrel{2}{\sim}$ & $\nexists$ & $\approx$ & - & $\infty$ & $\Rightarrow$ \\
\hline & 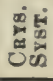 & $\cdot n$ & $\cdot m$ & : & $: \Xi$ & $\cdot m$ & 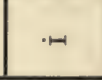 & 居包 \\
\hline & है & 일 & 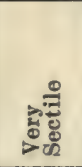 & 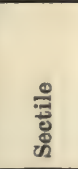 & 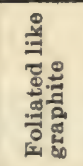 & 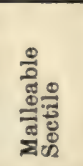 & 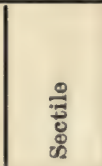 & 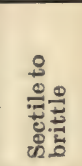 \\
\hline & 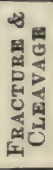 & 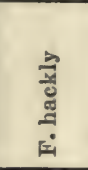 & $\begin{array}{l}\overline{\frac{\pi}{3}} \\
\frac{\pi}{8} \\
\frac{\pi}{0} \\
8 \\
8 \\
5\end{array}$ & 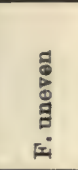 & $\begin{array}{l}\vec{J} \\
\text { : } \\
0\end{array}$ & 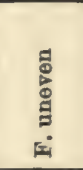 & & 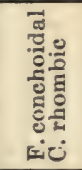 \\
\hline $\begin{array}{l}0 \\
0 \\
0\end{array}$ & 记 & 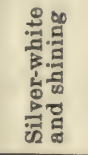 & 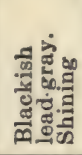 & 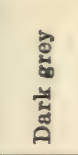 & $\frac{\text { जै }}{\text { लै }}$ & हैं & है & 马ु \\
\hline$E$ & ப- & $\frac{1}{\Delta}$ & ब & $\widehat{x}_{\infty}^{1}$ & 12 & స్ & ฝै & $d x^{*}$ \\
\hline$\frac{11}{11}$ & $\begin{array}{l}\text { 유 } \\
\text { ○ } \\
0\end{array}$ & 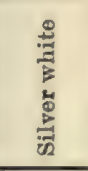 & 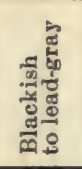 & 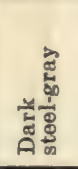 & 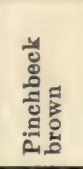 & 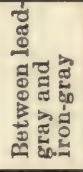 & 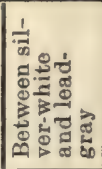 & 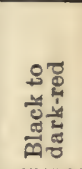 \\
\hline & 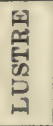 & 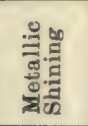 & 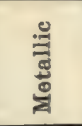 & 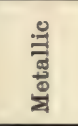 & 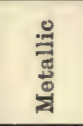 & 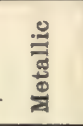 & है & 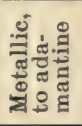 \\
\hline & 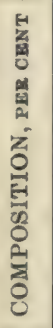 & 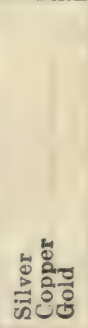 & 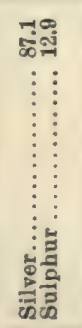 & 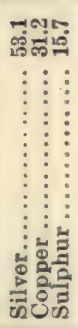 & 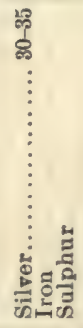 & 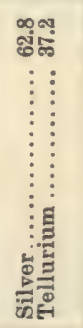 & 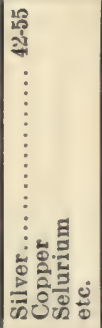 & 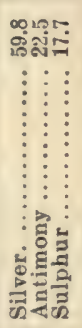 \\
\hline & 覴 & 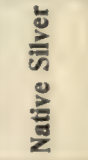 & 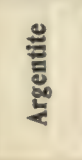 & 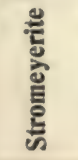 & 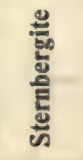 & $\begin{array}{l}\stackrel{y}{\mathscr{W}} \\
\stackrel{8}{3} \\
=\end{array}$ & 를 & है \\
\hline
\end{tabular}


Silver.

\begin{tabular}{|c|c|c|c|c|c|c|c|c|}
\hline & ठ் & 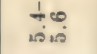 & क्ञ & $\stackrel{\text { సี }}{\square}$ & 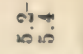 & 용 & $\stackrel{8}{\circ}$ & $b \omega^{\circ}$ \\
\hline & 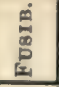 & - & - & -1 & - & $\Rightarrow$ & $\rightarrow$ & - \\
\hline & 通宫 & $\stackrel{-1}{=}$ & : & 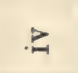 & $Z$ & $\cdot-\frac{8}{E}$ & . 1 & . \\
\hline & $\begin{array}{l}\frac{5}{5} \\
\frac{5}{6} \\
\frac{1}{6}\end{array}$ & 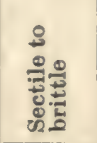 & 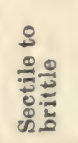 & 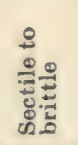 & & & & \\
\hline $\begin{array}{l}\overline{0} \\
\mathbf{w} \\
\mathbf{z} \\
\underline{z} \\
z \\
0\end{array}$ & 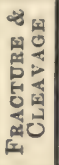 & 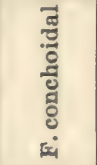 & 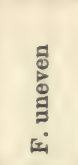 & 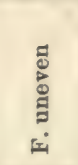 & 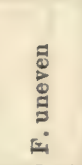 & 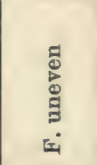 & 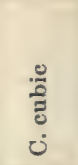 & 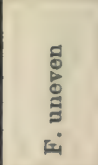 \\
\hline$\frac{a}{2}$ & 晃 & 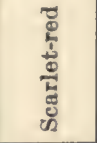 & 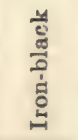 & है & 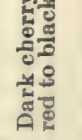 & 响 & 总 & 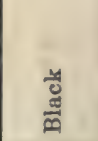 \\
\hline$\frac{2}{5}$ & 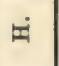 & ๙ & ล่ลี & ผลั & ง งกี & का & సे & ง \\
\hline$\underset{1}{a}$ & 융 & 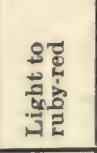 & 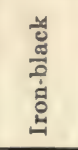 & 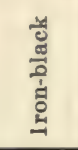 & 总 & 递焉 & 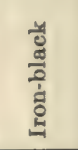 & 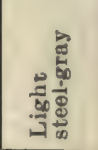 \\
\hline & 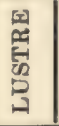 & 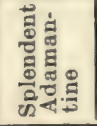 & 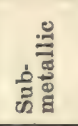 & 罰 & 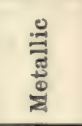 & 总 & 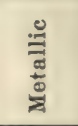 & 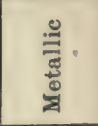 \\
\hline & 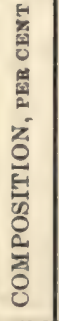 & 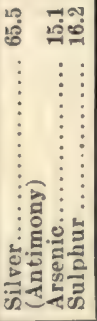 & 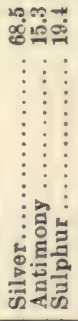 & 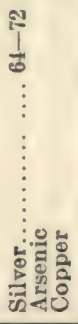 & 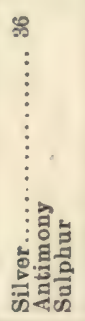 & 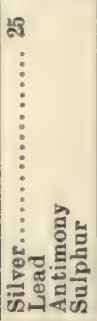 & 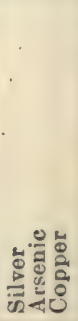 & 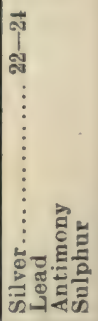 \\
\hline & 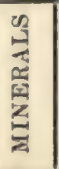 & 递 & 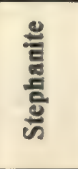 & 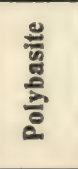 & 홀 & 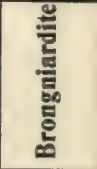 & $\begin{array}{l}\frac{y}{2} \\
\frac{0}{6} \\
\frac{2}{8}\end{array}$ & 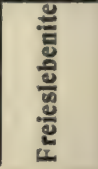 \\
\hline
\end{tabular}


Silver.

\begin{tabular}{|c|c|c|c|c|c|c|c|c|}
\hline & 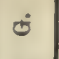 & $\stackrel{\infty}{-\infty}^{-\infty}$ & 18 & $\begin{array}{ll}\text { bis } \\
\text { of }\end{array}$ & ${ }_{\infty}^{\infty} \omega^{\infty}$ & $\begin{array}{l}\text { do } \\
\text { is }\end{array}$ & 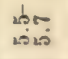 & \\
\hline & 兽 & -1 & $\therefore=$ & - & - & $\rightarrow$ & -1 & \\
\hline & 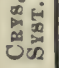 & 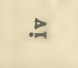 & & $\rightarrow$ & -1 & $m$ & $\bar{\nabla}$ & \\
\hline & 를 & 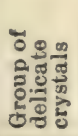 & & 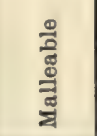 & 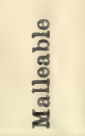 & & 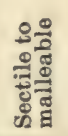 & \\
\hline $\begin{array}{l}0 \\
\text { w } \\
z \\
z \\
5 \\
z \\
0 \\
0\end{array}$ & 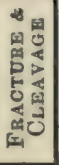 & 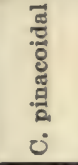 & 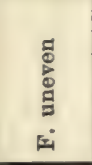 & 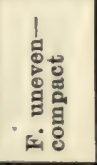 & 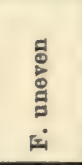 & 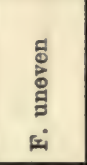 & 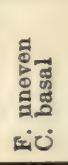 & \\
\hline d & 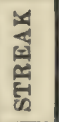 & ت્ّ & 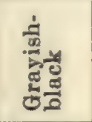 & 苞 & 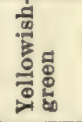 & 层 & 荧范 & \\
\hline & मี & N & & $\underbrace{\prime \prime}_{\infty}$ & àn & $1 \geq$ & & \\
\hline$\frac{5}{2}$ & 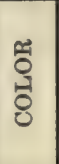 & 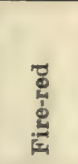 & 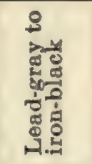 & 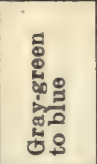 & 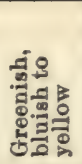 & 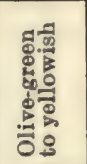 & ํㅜㄹ & \\
\hline 0 & 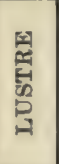 & है & 芵 & 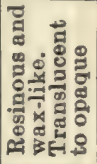 & 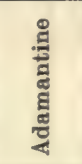 & 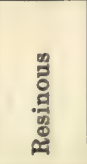 & 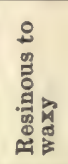 & \\
\hline & 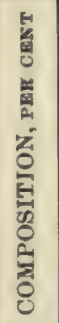 & 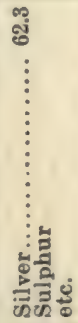 & 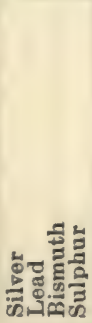 & 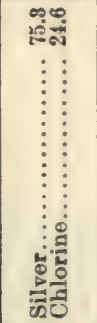 & 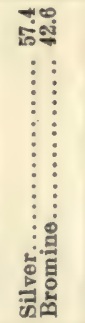 & 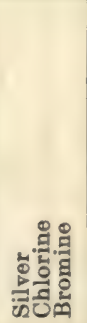 & 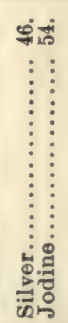 & \\
\hline & 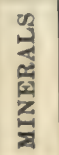 & $\begin{array}{l}\text { 를 } \\
\text { 를 }\end{array}$ & 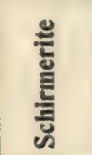 & 䒕 & 刻 & $\begin{array}{l}\text { 를 } \\
\text { 兽 }\end{array}$ & $\frac{9}{\frac{\pi}{2}}$ & \\
\hline
\end{tabular}


Copper.

\begin{tabular}{|c|c|c|c|c|c|c|c|c|}
\hline & ठீ & $\underset{\infty \infty \infty}{d ! \infty}$ & 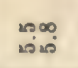 & $\stackrel{b}{\circ+\infty}$ & $\dot{\infty}_{\infty}^{\infty}$ & $\begin{array}{l}+109 \\
+400\end{array}$ & $\stackrel{0}{\circ}$ & $\stackrel{1}{120}$ \\
\hline & 鄀 & 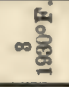 & ब่త్ & N & ㅊำ & ลื & -4 & の \\
\hline & 通苾 & 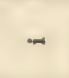 & $\vdots \beth$ & $:=$ & 恕 & $\sim$ & & \\
\hline & 홍 & 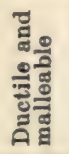 & 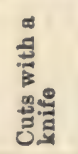 & 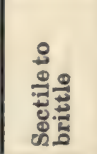 & 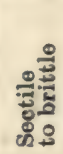 & 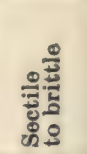 & & \\
\hline & 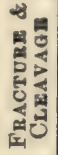 & 密 & $\begin{array}{l}\text { : } \\
\text { : } \\
\text { : }\end{array}$ & $\begin{array}{l}\Phi \\
\Phi \\
\vdots \\
\vdots \\
\vdots\end{array}$ & 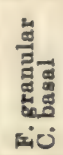 & 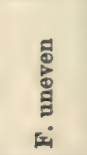 & 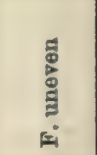 & 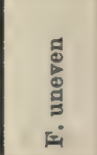 \\
\hline $\begin{array}{l}0 \\
0 \\
0 \\
0 \\
11\end{array}$ & 晃 & है & 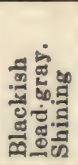 & 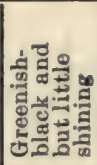 & 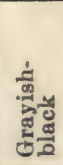 & 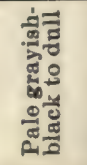 & है & 蒫 \\
\hline$=$ & I & $\frac{1}{N \infty}$ & సैं & )े & $\stackrel{1}{*}$ & (0) & ลึ่ & के \\
\hline $\begin{array}{l}\frac{c}{b} \\
\mathbf{a} \\
0 \\
0 \\
0\end{array}$ & 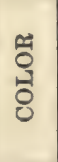 & 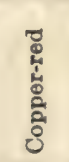 & 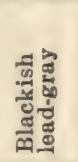 & 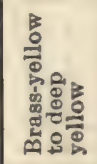 & 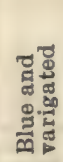 & 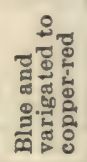 & 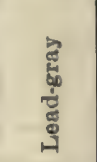 & 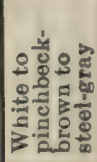 \\
\hline & 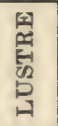 & 总 & 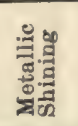 & 起 & 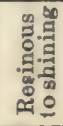 & 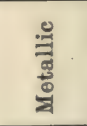 & 异 & 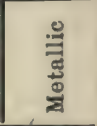 \\
\hline & 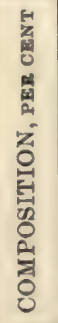 & हैं & 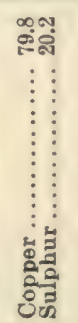 & 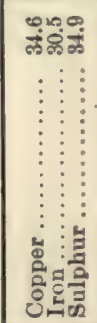 & 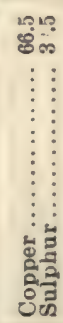 & 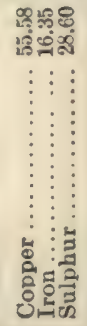 & 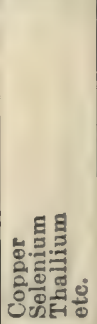 & 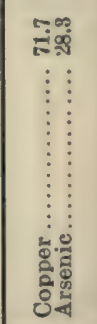 \\
\hline & 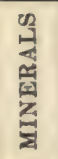 & $\begin{array}{l}\frac{5}{5} \\
\frac{1}{2} \\
\frac{1}{2}\end{array}$ & $\frac{\text { 흘 }}{\frac{3}{3}}$ & $\frac{\text { 른 }}{\frac{0}{8}}$ & 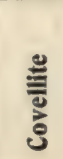 & 递 & 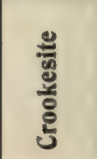 & 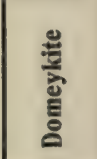 \\
\hline
\end{tabular}


Copper.

\begin{tabular}{|c|c|c|c|c|c|c|c|c|}
\hline & க் & $\stackrel{\oplus}{-}$ & 뭄 & $\begin{array}{l}\text { b8 } \\
\text { isos }\end{array}$ & 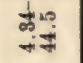 & $\stackrel{102}{0}$ & $\begin{array}{l}\text { bos } \\
\text { is }\end{array}$ & $\begin{array}{l}\text { สี } \\
\text { ه }\end{array}$ \\
\hline & 葍 & $\Rightarrow$ & $\underset{\sim}{*}$ & ob & -1 & $\sum_{\text {cato }}^{1}$ & $\infty$ & $\infty$ \\
\hline & 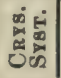 & $m$ & $-\infty$ & 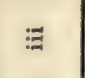 & $\vdots$ & $\rightarrow$ & 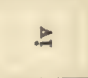 & $\triangleright$ \\
\hline & 递 & 营 & 竞 & 总 & 营 & 总 & 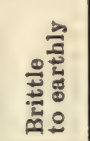 & 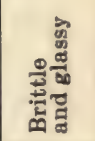 \\
\hline $\begin{array}{l}u \\
\vdots \\
z \\
z \\
z \\
0 \\
u\end{array}$ & 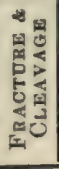 & 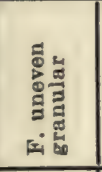 & 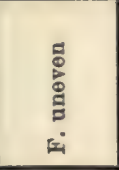 & 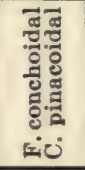 & 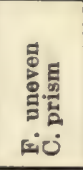 & 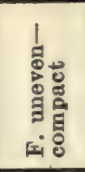 & 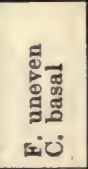 & 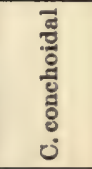 \\
\hline $\begin{array}{l}0 \\
1 \\
0 \\
\omega\end{array}$ & 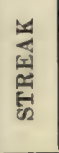 & 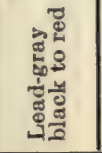 & 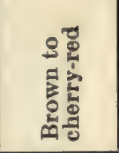 & 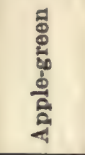 & 总 & 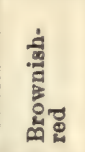 & 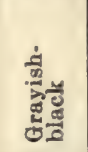 & Е \\
\hline 5 & | & ले & $\cos =$ & काँे & 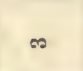 & $\stackrel{1}{\infty}$ & $\infty+$ & ๙ \\
\hline $\begin{array}{l}a \\
11 \\
a \\
0 \\
0 \\
0\end{array}$ & 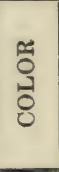 & 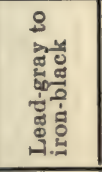 & 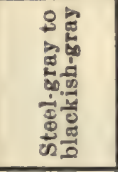 & 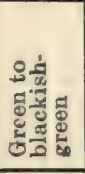 & 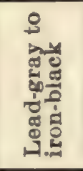 & 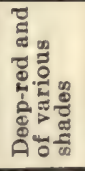 & 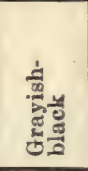 & $\frac{3}{3}$ \\
\hline & 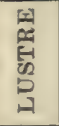 & 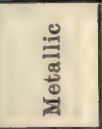 & 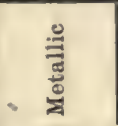 & 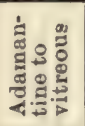 & 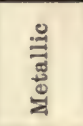 & 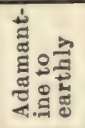 & 总泀 & 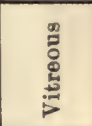 \\
\hline & 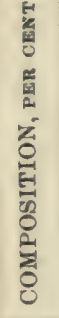 & 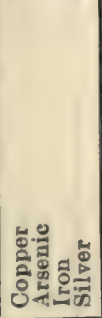 & 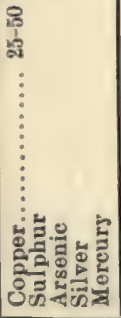 & 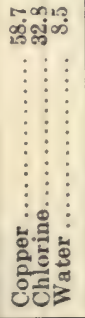 & 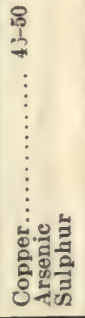 & 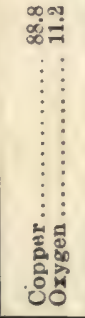 & 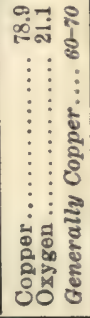 & (1) \\
\hline & 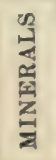 & 를 & 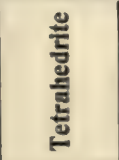 & 总 & 题 & 突 & 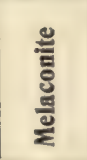 & 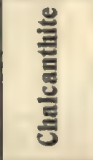 \\
\hline
\end{tabular}


Copper.

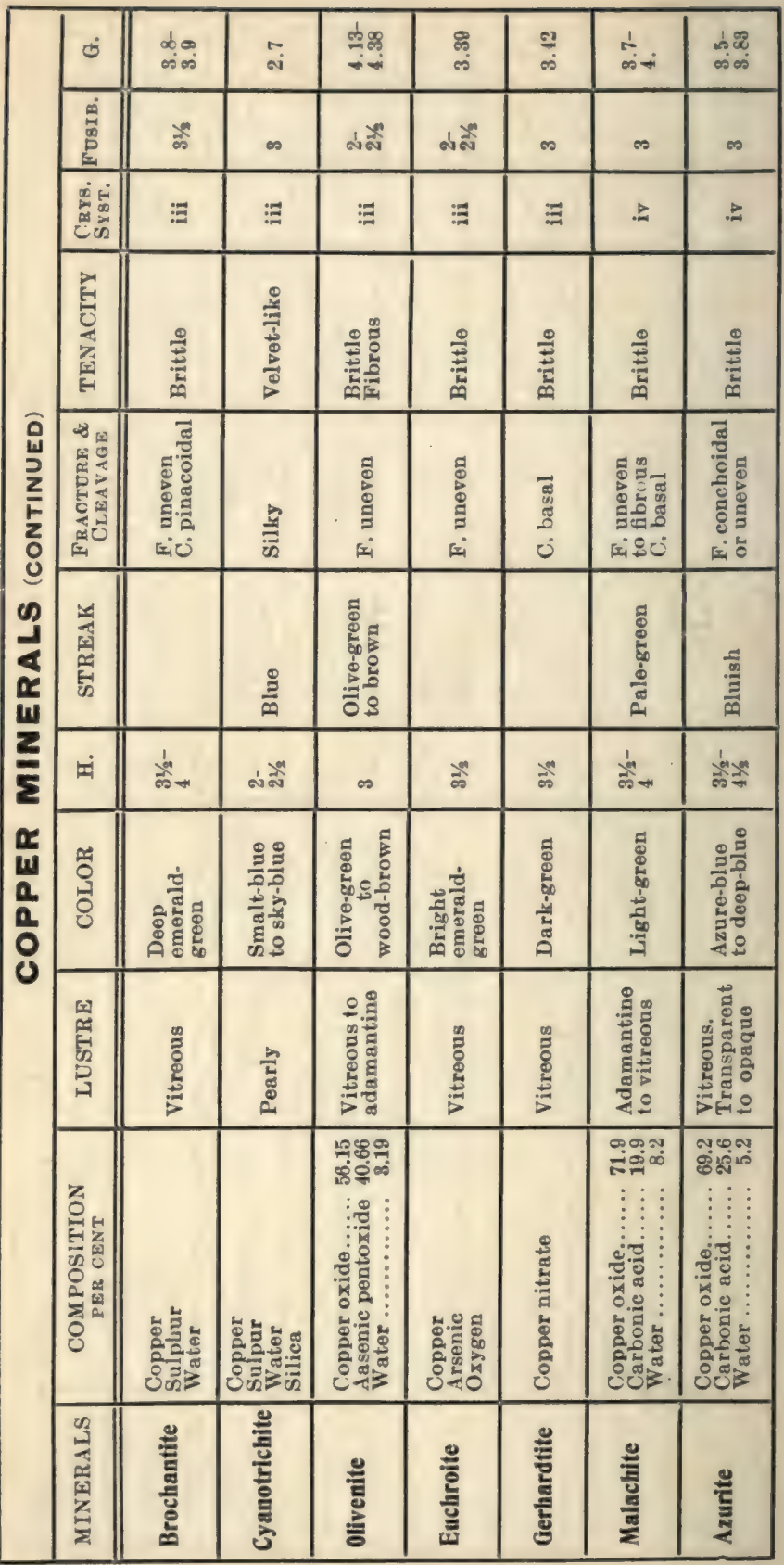


Copper-Lead.

\begin{tabular}{|c|c|c|c|c|c|c|c|c|c|}
\hline & க் & 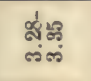 & बंग & \multirow{11}{*}{ 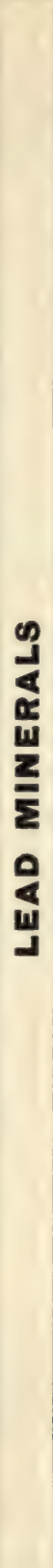 } & க் & $\stackrel{68}{=}$ & 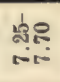 & $\underset{\substack{+\infty \\
=\infty}}{ }$ & $\underset{\infty}{\infty}$ \\
\hline & 葛 & 0 & $\bullet$ & & 若 & 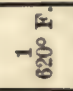 & a & ๙ & $\approx$ \\
\hline & 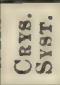 & 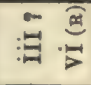 & 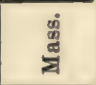 & & 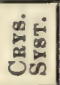 & r & $m$ & $\pi$ & $-\infty$ \\
\hline & 总 & 焉 & 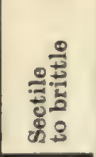 & & 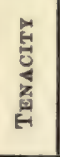 & 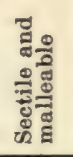 & 䒿 & 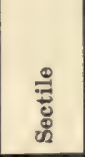 & 莺 \\
\hline 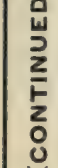 & 递圈 & 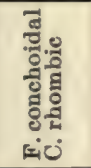 & 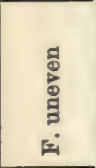 & & 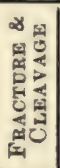 & 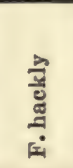 & 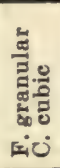 & 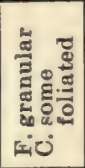 & 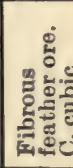 \\
\hline$\frac{9}{4}$ & 蝁 & 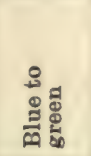 & 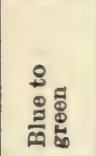 & & 幽 & 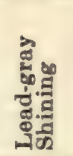 & 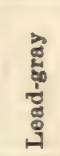 & $\begin{array}{l}\text { 芦 } \\
\text { 总 }\end{array}$ & 胥 \\
\hline$\frac{2}{5}$ & 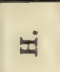 & 10 & $\omega *$ & & 皇 & $\nexists$ & ลें & ते & के \\
\hline $\begin{array}{l}\frac{x}{w} \\
\frac{w}{0} \\
\frac{0}{0} \\
0\end{array}$ & 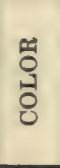 & 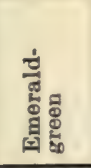 & 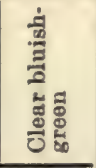 & & $\begin{array}{l}\text { 。ี } \\
\stackrel{3}{0} \\
8\end{array}$ & 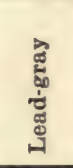 & 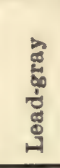 & 递 & 急 \\
\hline & 四 & 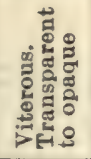 & 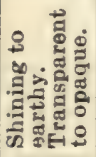 & & 䇥 & $\overline{\bar{\Xi}}$ & 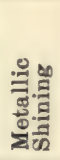 & 元 & 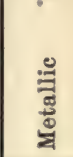 \\
\hline & 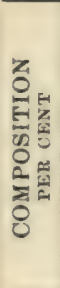 & 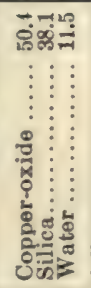 & 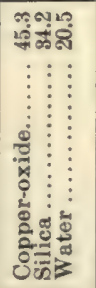 & & 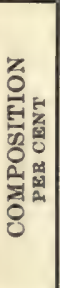 & 苟高 & 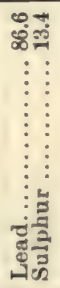 & 量 & 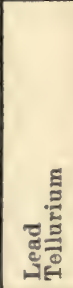 \\
\hline & 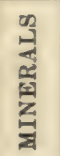 & 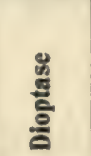 & 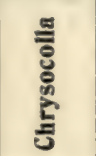 & & 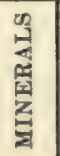 & 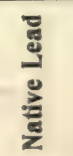 & 팰 & 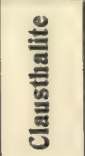 & 农 \\
\hline
\end{tabular}


Lead.

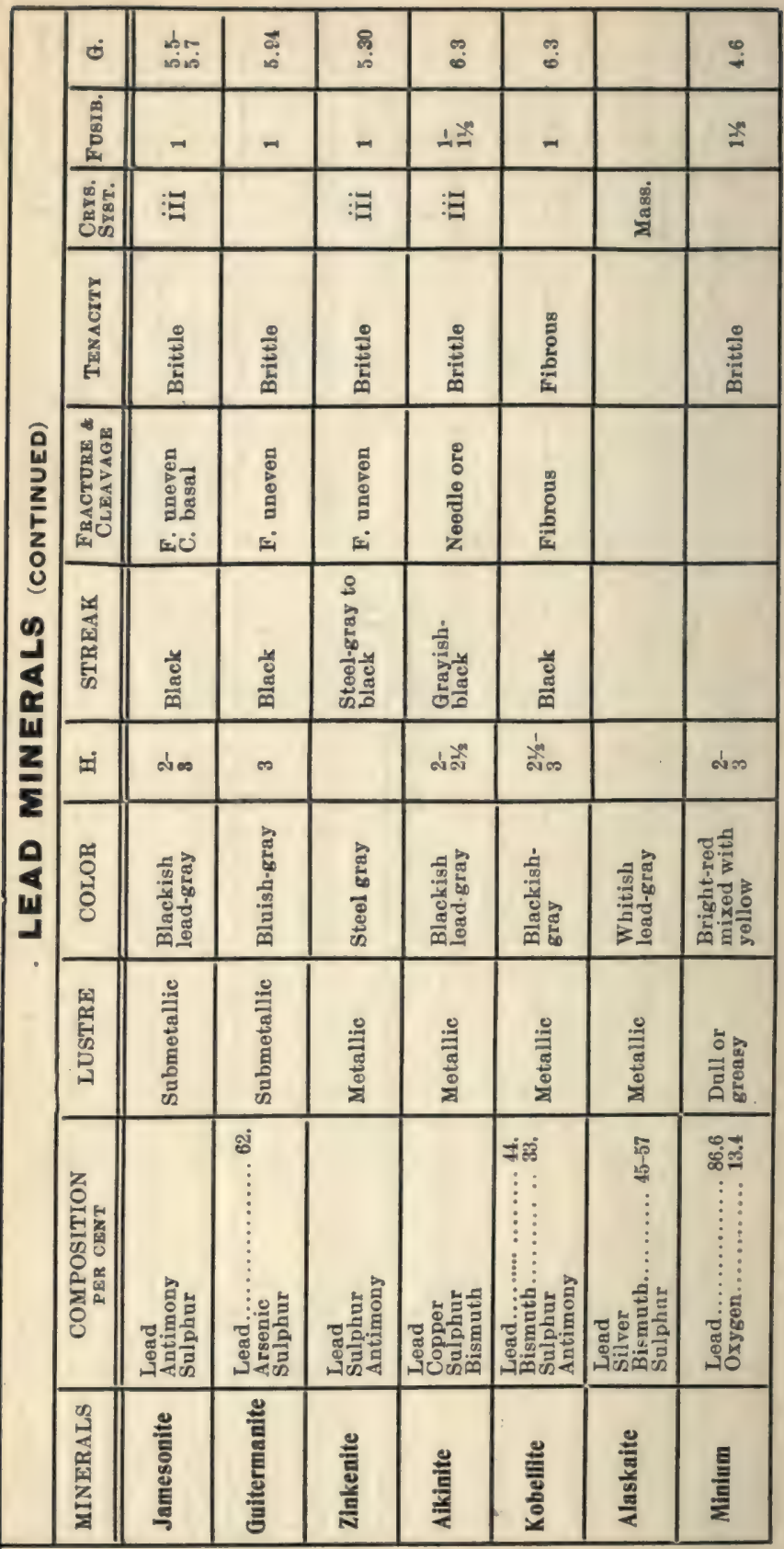


Lead.

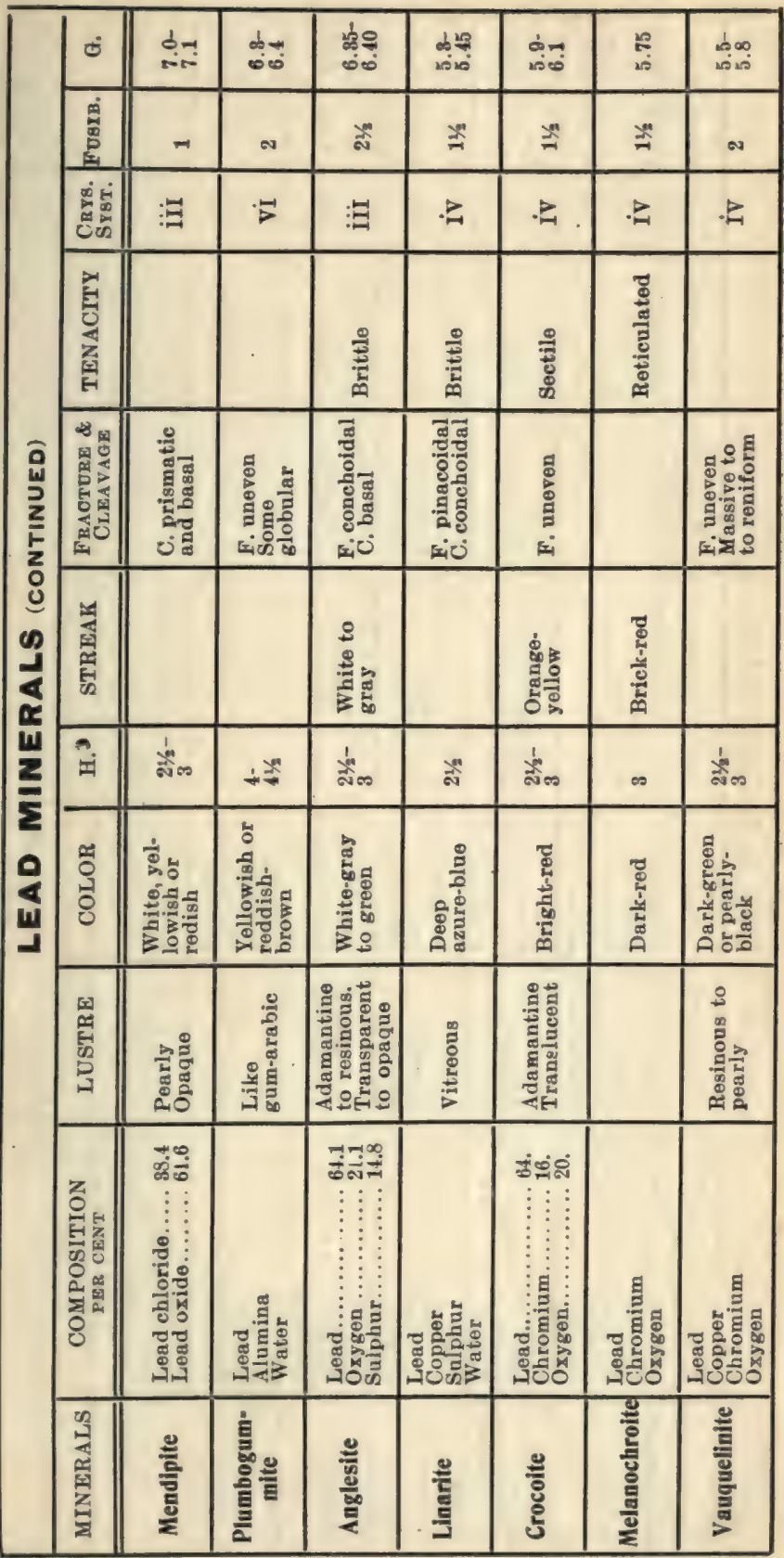


Lead.

\begin{tabular}{|c|c|c|c|c|c|c|c|c|}
\hline & $\mathbb{C}^{\circ}$ & $\stackrel{8}{6}$ & $d_{-\infty}^{d-\infty}$ & 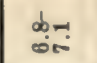 & च" & d: & $\because \overline{0}$ & $\frac{0}{00}$ \\
\hline & 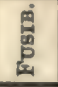 & Co & ลื่ & N & $\approx$ & $\nexists$ & $\nexists^{*}$ & $\underset{*}{*}$ \\
\hline & 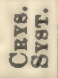 & : & $:=$ & 岸 & 5 & 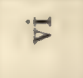 & $: \Xi$ & : $: \nexists$ \\
\hline & 률 & 总 & 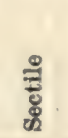 & 䒿 & 营 & - 咅 & 莣 & 营 \\
\hline $\begin{array}{l}6 \\
w \\
2 \\
z \\
z \\
z\end{array}$ & 圆造 & 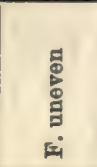 & $\begin{array}{l}\text { है } \\
\text { है } \\
\text { है } \\
\text { घं }\end{array}$ & 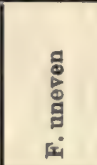 & 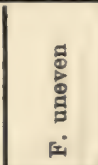 & 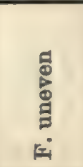 & 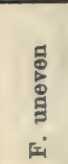 & 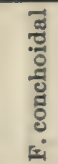 \\
\hline $\begin{array}{l}0 \\
0 \\
a \\
0\end{array}$ & 礐 & 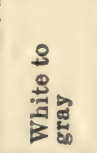 & 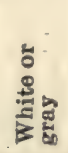 & $\frac{8}{30}$ & 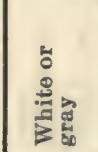 & 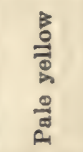 & & 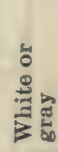 \\
\hline$\underline{2}$ & ت्ن & $\stackrel{1}{*}$ & 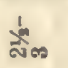 & खें & ลึं & ลें & ले & का \\
\hline a & 병 & 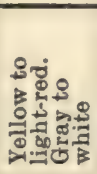 & 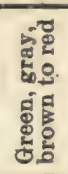 & 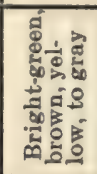 & 总 & 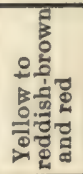 & 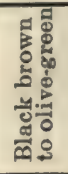 & 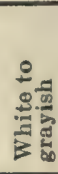 \\
\hline & 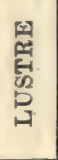 & 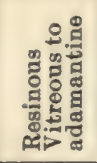 & $\begin{array}{l}\text { m } \\
\text { : } \\
\text { : }\end{array}$ & 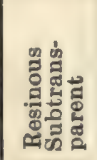 & 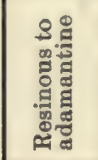 & 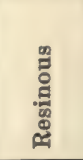 & 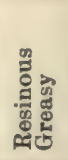 & 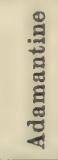 \\
\hline & 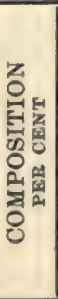 & 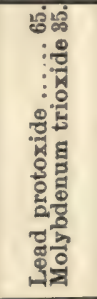 & 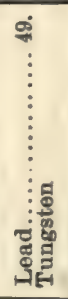 & 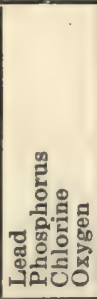 & 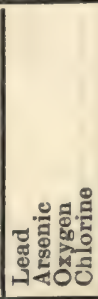 & 밀 & 를 & 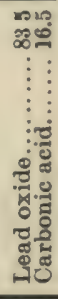 \\
\hline & 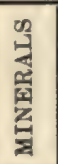 & 离 & $\frac{3}{\frac{\pi}{*}}$ & $\begin{array}{l}\text { 홀 } \\
\text { 흔 } \\
\text { 틀 } \\
\text { 롤 }\end{array}$ & 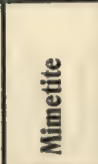 & 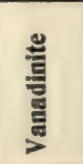 & 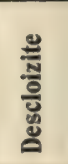 & $\begin{array}{l}\text { ㅂㅗㅐ } \\
\text { 崫 } \\
\text { త }\end{array}$ \\
\hline
\end{tabular}


Zinc.

\begin{tabular}{|c|c|c|c|c|c|c|c|c|c|}
\hline & $0^{\circ}$ & $\underset{\infty}{\operatorname{dey}}$ & $\overrightarrow{+}$ & 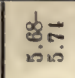 & $\begin{array}{l}\text { के } \\
\text { oे }\end{array}$ & $\operatorname{de}^{2} \stackrel{4}{-1}$ & $\begin{array}{c}\infty \infty \\
\infty \\
\infty\end{array}$ & ${ }_{\infty}^{\infty}$ & $\begin{array}{l}800 \\
807 \\
\infty \\
\infty\end{array}$ \\
\hline & 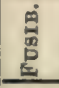 & is & 10 & 0 & 20 & 10 & $\infty$ & an & $\infty$ \\
\hline & 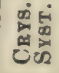 & . In & 5 & 15 & 洁 & 际包 & & D & • \\
\hline & E⿱艹 & 总 & 䒿 & हึّ & 忢 & 売 & & 营 & 营 \\
\hline & 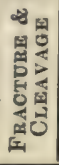 & 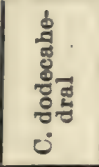 & 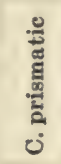 & 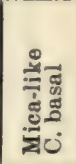 & 象 & 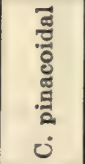 & & 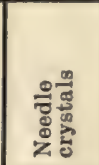 & 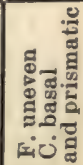 \\
\hline $\begin{array}{c}0 \\
0 \\
0\end{array}$ & 氹 & 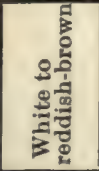 & 范 & 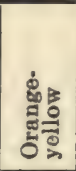 & 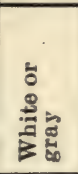 & 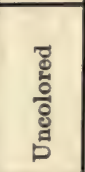 & & & 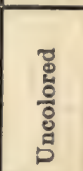 \\
\hline 2 & $\ddot{I}$ & $\frac{1}{x^{\infty}}$ & लें & $+\infty$ & ลे & 10 & ฟลี่ & N & 100 \\
\hline $\begin{array}{l}2 \\
0 \\
\frac{1}{2}\end{array}$ & $\begin{array}{l}\text { 유 } \\
\text { 웅 }\end{array}$ & 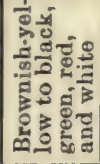 & 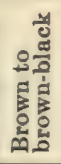 & 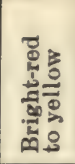 & 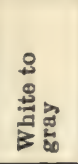 & 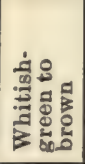 & 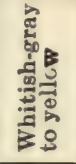 & 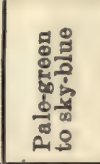 & 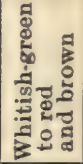 \\
\hline & 里 & 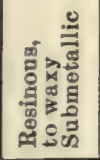 & 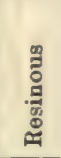 & 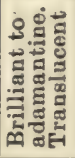 & 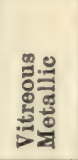 & 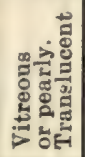 & 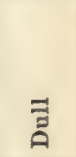 & $\begin{array}{l}\vec{E} \\
\stackrel{\Xi}{\Xi} \\
\text { م }\end{array}$ & 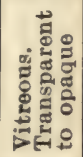 \\
\hline & 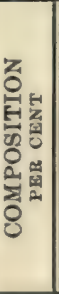 & 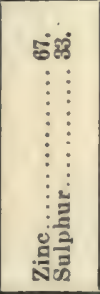 & 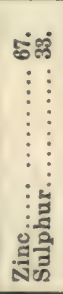 & 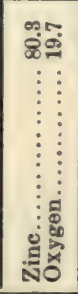 & 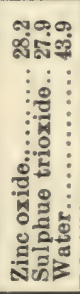 & 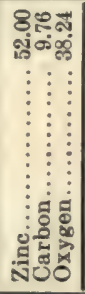 & 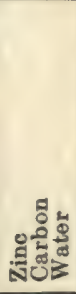 & 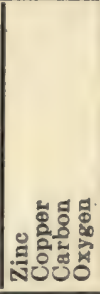 & 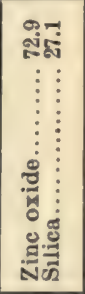 \\
\hline & 幂 & $\frac{\frac{\dddot{y}}{5}}{\frac{y}{E}}$ & $\frac{3}{3}$ & 를 & 흘 & 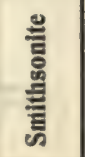 & $\begin{array}{l}\text { 틍 } \\
\text { 흘 } \\
\text { 를 }\end{array}$ & 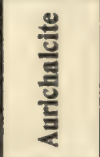 & 总 \\
\hline
\end{tabular}


Zinc-Cobalt and Nickel.

\begin{tabular}{|c|c|c|c|c|c|c|c|c|c|}
\hline சं & 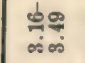 & - & & ن் & $\stackrel{b}{\infty}$ & 8 & $\frac{+0}{0}$ & den & बू. \\
\hline 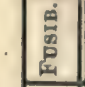 & is & $\infty$ & & 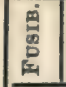 & s & $\underset{\sim}{ \pm}$ & ลั & d' & N \\
\hline 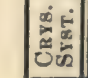 & $: \equiv$ & $\cdot-1$ & & 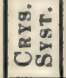 & •ロ & 止正 & 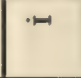 & • & $\ddot{D}$ \\
\hline 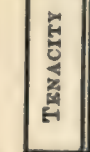 & 苛 & 营 & & $\begin{array}{l}\text { है } \\
\text { है } \\
\text { दू } \\
\text { ह }\end{array}$ & 竎 & 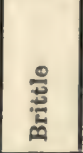 & 竎 & 宽 & 胥 \\
\hline 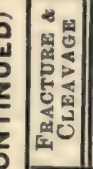 & 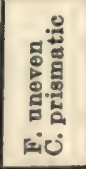 & 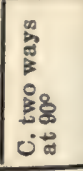 & $\frac{0}{\alpha}$ & 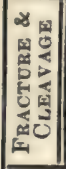 & 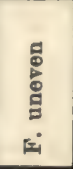 & 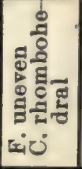 & 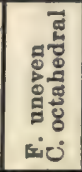 & 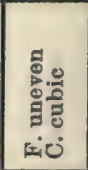 & $\begin{array}{l}\text { है } \\
0 \\
0 \\
0\end{array}$ \\
\hline 學 & 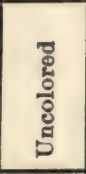 & 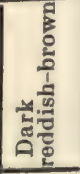 & $\frac{\omega}{2}$ & 댄 & 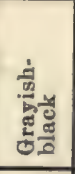 & 造 & 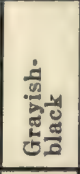 & 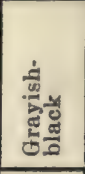 & 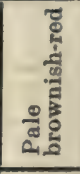 \\
\hline$\ddot{\theta}$ & $\neq$ & 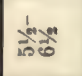 & $\frac{M}{0}$ & |खं & ลี & काँ & 孚 & $x^{*}$ & 的跑 \\
\hline 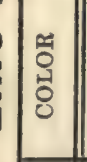 & 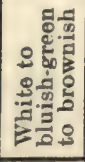 & 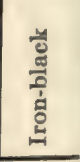 & $\begin{array}{l}z \\
0 \\
2\end{array}$ & 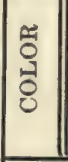 & 点 & 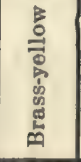 & 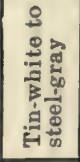 & 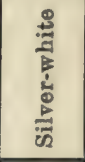 & 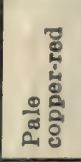 \\
\hline 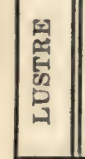 & 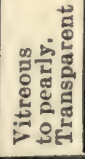 & 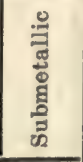 & $\frac{1}{0}$ & 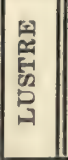 & 总 & 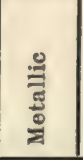 & 屏 & 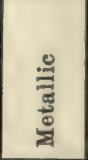 & 兽 \\
\hline 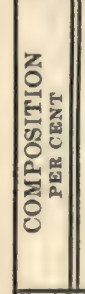 & 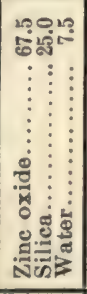 & 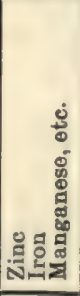 & & 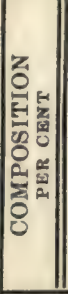 & 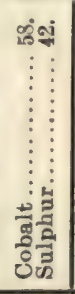 & 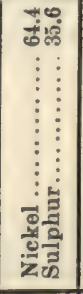 & 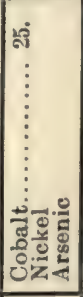 & 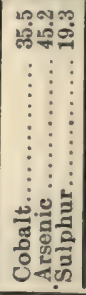 & 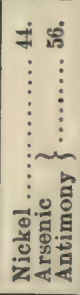 \\
\hline 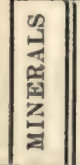 & 를 & 总 & & 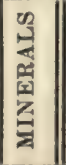 & $\begin{array}{l}\frac{9}{3} \\
\text { 뜰 }\end{array}$ & 흘 & $\begin{array}{l}\stackrel{3}{\underline{E}} \\
\text { है }\end{array}$ & 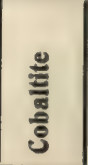 & 预 \\
\hline
\end{tabular}


Cobalt and Nickel.

\begin{tabular}{|c|c|c|c|c|c|c|c|c|c|}
\hline \multirow{4}{*}{$\begin{array}{l}0 \\
\text { w } \\
2 \\
z \\
z \\
z \\
0 \\
0\end{array}$} & రீ & त् & $\begin{array}{l}\text { be } \\
\text { 100 }\end{array}$ & $\stackrel{0}{70}$ & & $\begin{array}{l}-100 \\
-\infty=0\end{array}$ & बis & abro & $\begin{array}{l}\text { ब̦do } \\
\text { sion }\end{array}$ \\
\hline & 蛋 & o & $\infty$ & $\overrightarrow{0}-$ & & $\%$ & สู & 0 & 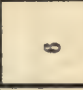 \\
\hline & 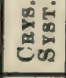 & 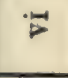 & $\cdot$ & $\cdot \sim$ & & $\stackrel{\text { 兽 }}{z}$ & $z$ & $\stackrel{\text { D }}{\stackrel{0}{\Xi}}$ & 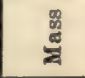 \\
\hline & 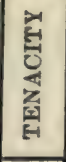 & 营 & 䒿 & 壱 & & 㒮 & 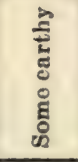 & & है \\
\hline $\begin{array}{l}0 \\
0 \\
0 \\
0 \\
0\end{array}$ & 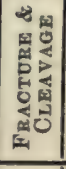 & & 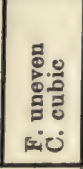 & 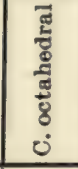 & 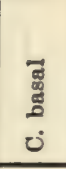 & & 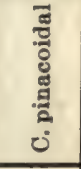 & 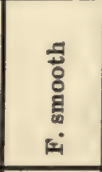 & $\begin{array}{l}5 \\
8 \\
0 \\
0\end{array}$ \\
\hline$\frac{2}{\frac{2}{2}}$ & 章 & $\bullet$ & $\frac{\ddot{E}}{\frac{\pi}{0}}$ & 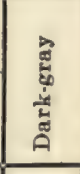 & & & 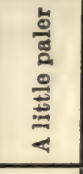 & & \\
\hline$\frac{y}{0}$ & 7 & $\sum_{1000}^{1}$ & में & 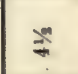 & & ล่సే & $\mathbb{X}_{\infty}$ & ठื & $\dot{\infty}$ \\
\hline 2 & 명 & 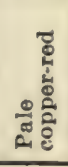 & 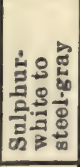 & 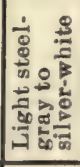 & 兽。 & 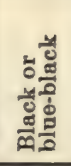 & 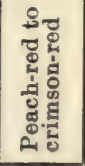 & 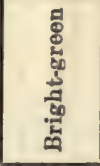 & 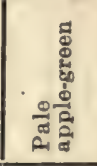 \\
\hline$\frac{1}{0}$ & 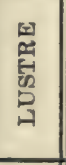 & 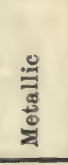 & 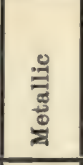 & 를 & 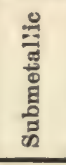 & 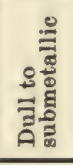 & 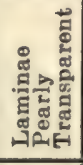 & 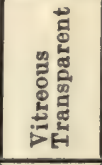 & 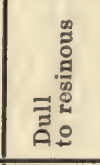 \\
\hline & 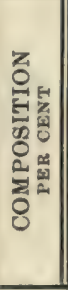 & 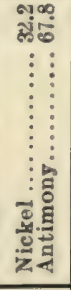 & 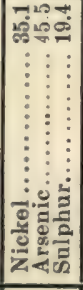 & 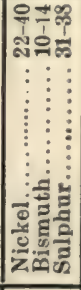 & 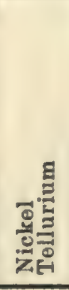 & 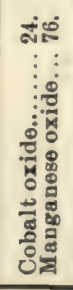 & 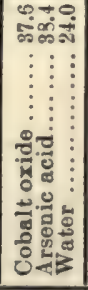 & 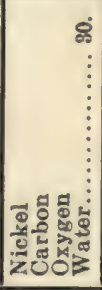 & 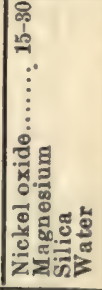 \\
\hline & 资 & $\begin{array}{l}\text { 를 } \\
\text { 휼 } \\
\text { 를 }\end{array}$ & 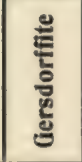 & 总 & $\frac{\text { 를 }}{\frac{0}{\frac{0}{2}}}$ & $\begin{array}{l}\frac{\Xi}{\bar{\Xi}} \\
\frac{\text { के }}{<}\end{array}$ & 롤 & 帝 & 를 \\
\hline
\end{tabular}


Mercury.

\begin{tabular}{|c|c|c|c|c|c|c|c|c|}
\hline & ம் & 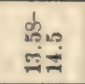 & 10 & $\underset{\infty}{\infty}$ & $\infty$ & $\begin{array}{l}\mathscr{8} \\
\infty \\
\infty\end{array}$ & $\stackrel{\frac{9}{2}}{0}$ & \\
\hline & 惹 & : & $\gg 0$ & $-\dot{0}$ & $\overrightarrow{0}$ & -1 & -3 & \\
\hline & 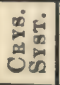 & - - & 䛌 & $m$ & $\begin{array}{l}\text { 总 } \\
\text { 巴્心 }\end{array}$ & 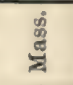 & $:=$ & \\
\hline & 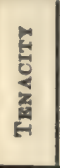 & 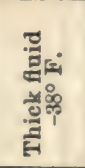 & 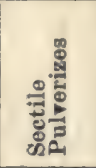 & & & & 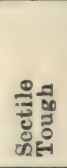 & 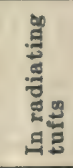 \\
\hline & 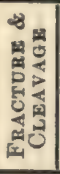 & & 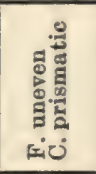 & 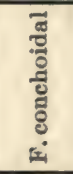 & 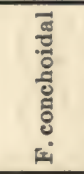 & 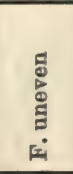 & $\begin{array}{l}\bar{\varpi} \\
\overline{0} \\
\overline{0} \\
\vdots \\
\vdots \\
\end{array}$ & 总 \\
\hline$\frac{\infty}{2}$ & 娄 & & 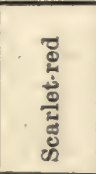 & $\begin{array}{l}\text { है } \\
\text { क्ञ }\end{array}$ & $\begin{array}{l}\text { 总 } \\
\text { 㟧 }\end{array}$ & 常 & 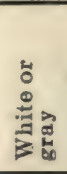 & 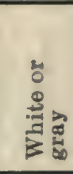 \\
\hline $\bar{\Sigma}$ & 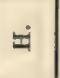 & & ผลิ & $x^{*}$ & సे" & $\infty$ & 血 & - \\
\hline$\frac{2}{2}$ & $\begin{array}{l}\approx \\
0 \\
0 \\
0\end{array}$ & 总 & 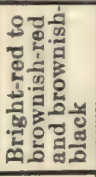 & 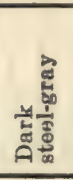 & 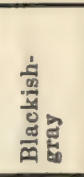 & 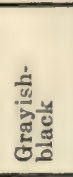 & 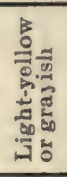 & $\frac{0}{3}$ \\
\hline & 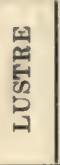 & $\begin{array}{l}\vec{E} \\
\text { कूँ } \\
\text { की } \\
\text { की }\end{array}$ & 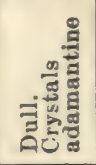 & 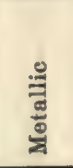 & 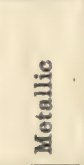 & 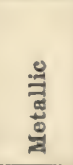 & 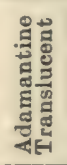 & ऊี \\
\hline & 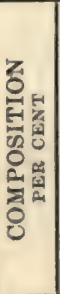 & 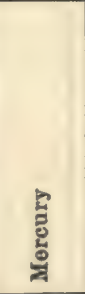 & 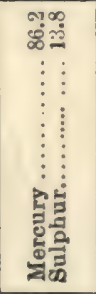 & 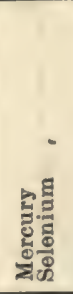 & 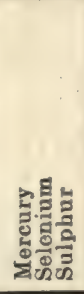 & 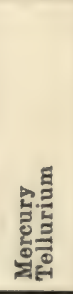 & 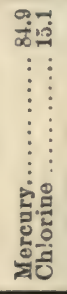 & 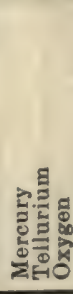 \\
\hline & 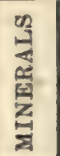 & 4 & 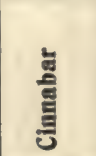 & 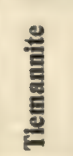 & 冚 & $\begin{array}{l}\frac{\pi}{5} \\
\frac{\pi}{5} \\
\frac{5}{3}\end{array}$ & $\begin{array}{l}\bar{\Xi} \\
\overline{\bar{z}} \\
\bar{\Xi}\end{array}$ & 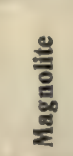 \\
\hline
\end{tabular}


Iron.

\begin{tabular}{|c|c|c|c|c|c|c|c|c|c|c|}
\hline & $0^{\circ}$ & $\underset{i}{\infty} \rightarrow \infty$ & dos & 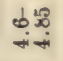 & تص & $\begin{array}{l}\frac{1}{6} \infty \\
100\end{array}$ & $\begin{array}{l}\alpha E \\
0 \infty\end{array}$ & 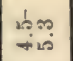 & bo & $\begin{array}{l}d \\
0.5\end{array}$ \\
\hline & 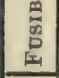 & $\infty$ & तั่ & సิ่n & ลึ่ & N. & 20 & $\infty$ & $\infty$ & $\infty$ \\
\hline & 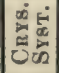 & • & $m$ & : & $\overrightarrow{>}$ & $\vdots$ & 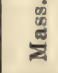 & 50 & 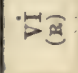 & $\mapsto$ \\
\hline & 递 & 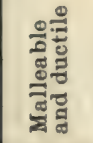 & 䒯 & 营 & 䒯 & 䓪 & 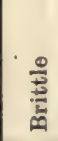 & 营 & 总 & 营 \\
\hline & 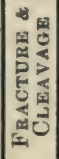 & 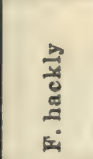 & 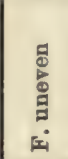 & 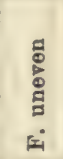 & 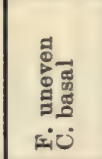 & 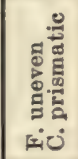 & 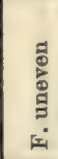 & 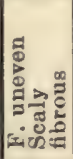 & 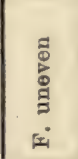 & 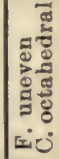 \\
\hline$\frac{a}{\alpha}$ & 部 & 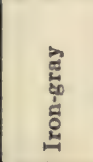 & 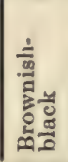 & 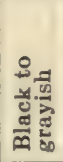 & 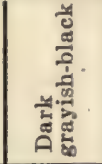 & 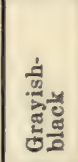 & 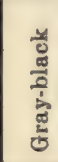 & 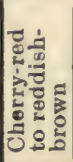 & 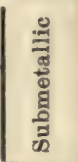 & $\begin{array}{l}\text { हैं } \\
\frac{\varpi}{\text { ติ }}\end{array}$ \\
\hline 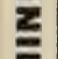 & $\dot{1}$ & $\neq$ & कृष & bढे & केస & $x_{\text {ine }}^{1}$ & ม่⿻ & 1000 & be & 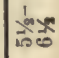 \\
\hline $\begin{array}{l}2 \\
0 \\
0\end{array}$ & $\begin{array}{l}\text { 때 } \\
\text { ఏ } \\
0 \\
0\end{array}$ & 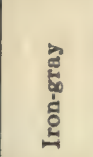 & 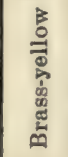 & 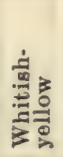 & 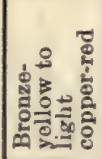 & 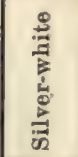 & 章 & 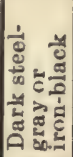 & 늘 & 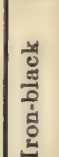 \\
\hline & 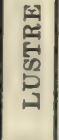 & 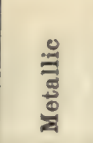 & 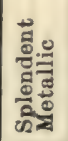 & 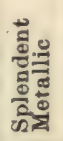 & 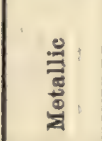 & कै & 芭 & 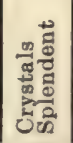 & 离 & 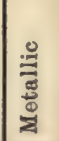 \\
\hline & 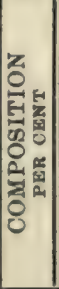 & 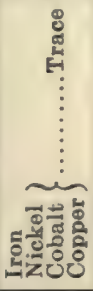 & 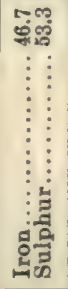 & 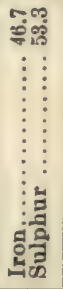 & 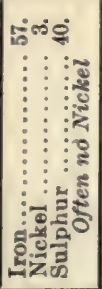 & 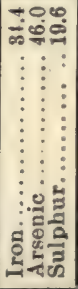 & 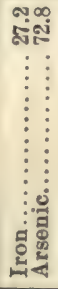 & 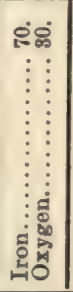 & 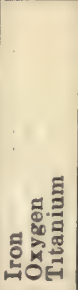 & 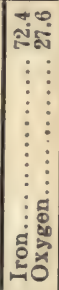 \\
\hline & 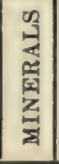 & 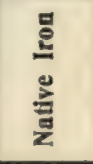 & $\frac{9}{2}$ & 를 & 흔 & 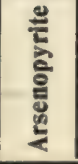 & 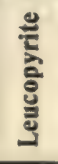 & 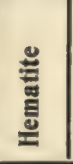 & 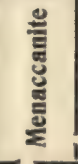 & 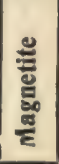 \\
\hline
\end{tabular}


Iron.

\begin{tabular}{|c|c|c|c|c|c|c|c|c|c|}
\hline & $\ddot{~}$ & क्ष्रे० & do & $0=$ & $\stackrel{8}{-1}$ & $\underset{x \rightarrow 0}{1}$ & $\begin{array}{l}+120 \\
+\infty \infty \\
\infty\end{array}$ & $\begin{array}{l}\text { वर्ष } \\
\text { cici }\end{array}$ & $\begin{array}{l}1 \\
-\infty \\
\infty\end{array}$ \\
\hline & 象 & $\infty$ & $\bullet$ & bo & $1 x$ & क के & 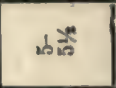 & فำ & $\neq \infty$ \\
\hline & 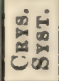 & $m$ & ๕ & 洁 & $\nexists$ & $\geq$ & : & 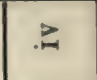 & $P \theta$ \\
\hline & $\sum_{\substack{y \\
0}}^{\infty}$ & 递 & 吾 & 葴 & 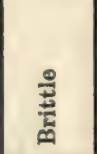 & 莡 & 营 & 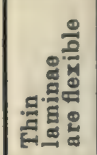 & 衰 \\
\hline $\begin{array}{l}0 \\
\text { w } \\
\frac{1}{2} \\
\frac{2}{2}\end{array}$ & 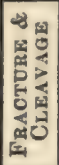 & 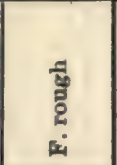 & 啇 & 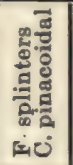 & 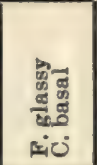 & 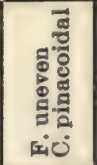 & 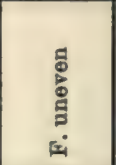 & 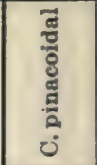 & 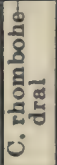 \\
\hline $\begin{array}{l}0 \\
0 \\
0 \\
1 \\
0 \\
0\end{array}$ & 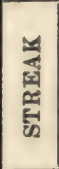 & 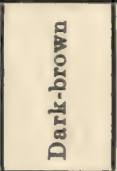 & 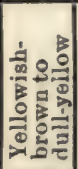 & 通 & & 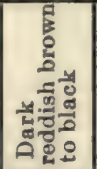 & 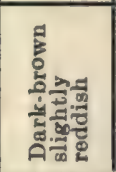 & $\frac{\frac{a}{5}}{\frac{9}{3}}$ & $\begin{array}{l}0 \\
0 \\
0 \\
0 \\
0 \\
0 \\
0\end{array}$ \\
\hline 2 & घं & in & a & ม่า & os & 2 $\operatorname{xin}$ & טלמי & $\pm_{\infty}^{\infty}$ & $\infty x$ \\
\hline $\begin{array}{l}2 \\
2 \\
0 \\
0\end{array}$ & $\begin{array}{l}\approx \\
0 \\
0 \\
0\end{array}$ & 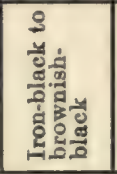 & 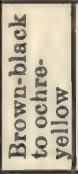 & 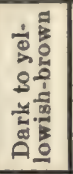 & 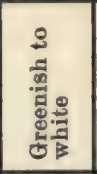 & 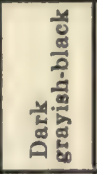 & 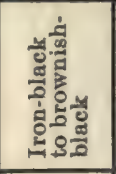 & 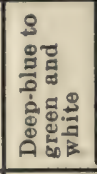 & 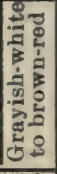 \\
\hline & 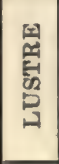 & 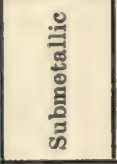 & 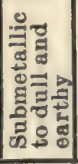 & 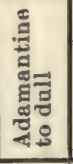 & 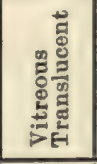 & 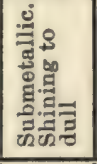 & 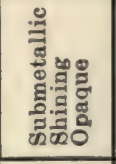 & 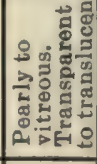 & 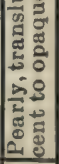 \\
\hline & 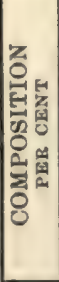 & 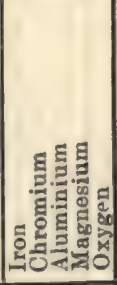 & 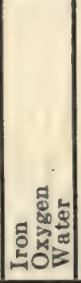 & 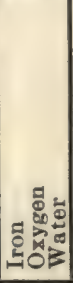 & 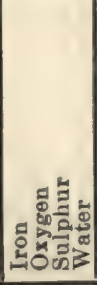 & 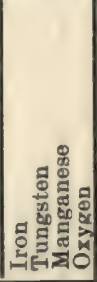 & 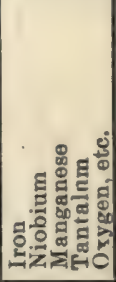 & 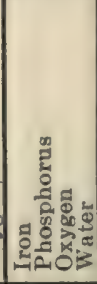 & 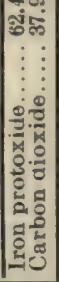 \\
\hline & 㗭 & 를 & 总 & 롤 & $\frac{3}{\frac{3}{5}}$ & 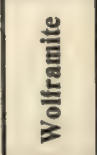 & 高 & $\stackrel{巳}{\stackrel{\Xi}{E}}$ & 竎 \\
\hline
\end{tabular}


Manganese.

\begin{tabular}{|c|c|c|c|c|c|c|c|c|c|}
\hline & ல் & $\stackrel{\infty}{\rightarrow}$ & $\stackrel{\infty}{-}$ & $d-$ & $\begin{array}{l}\text { do } \\
\text { os - }\end{array}$ & & $\begin{array}{l}\text { ine } \\
\text { io }\end{array}$ & $\begin{array}{l}+\infty \\
\sin 0\end{array}$ & $\frac{1}{x^{\circ} \infty}$ \\
\hline & 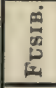 & $\infty$ & $\infty$ & $\infty$ & में & & खै & a & $\Xi$ \\
\hline & 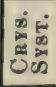 & -1 & : & $\begin{array}{l}\stackrel{0}{0} \\
\stackrel{\Xi}{\sharp} \\
\ddot{z}\end{array}$ & 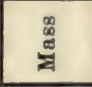 & & : : & : & $\overrightarrow{>}$ \\
\hline & 荘 & & 产 & 节 & 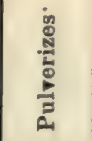 & 胥胥 & 莺 & 晜 & 营 \\
\hline $\boldsymbol{\infty}$ & 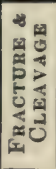 & \begin{tabular}{l|}
0 \\
0 \\
0 \\
0 \\
0
\end{tabular} & 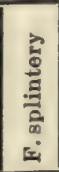 & $\begin{array}{l}\text { 퓽 } \\
0 \\
0 \\
0 \\
0\end{array}$ & है & & $\begin{array}{l}\vec{~} \\
\text { ळू } \\
\text { : } \\
\dot{0}\end{array}$ & 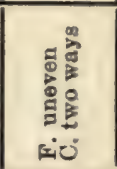 & $\begin{array}{l}\frac{8}{8} \\
\frac{8}{6} \\
\frac{8}{4} \\
0\end{array}$ \\
\hline $\begin{array}{l}a \\
2 \\
2\end{array}$ & 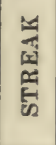 & & 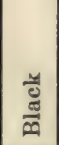 & 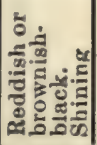 & 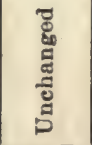 & & 造。 & 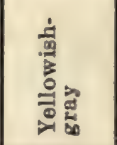 & \\
\hline $\mathbf{w}$ & $\ddot{\square}$ & $\therefore \infty$ & ผ & the & tie & -1 & 20 & bु & ले \\
\hline$\frac{11}{2}$ & 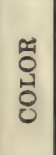 & 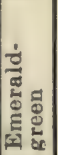 & 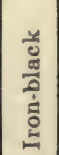 & 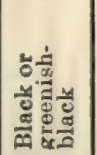 & 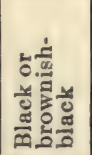 & 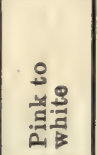 & 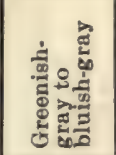 & 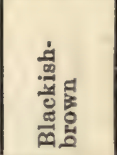 & 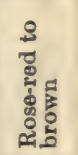 \\
\hline 5 & 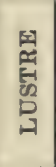 & 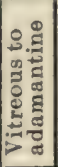 & 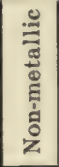 & 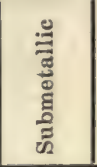 & 春吾 & & 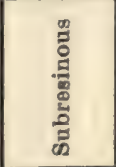 & 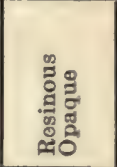 & 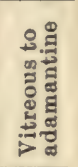 \\
\hline & 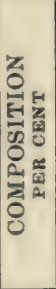 & 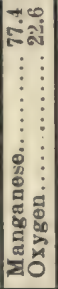 & 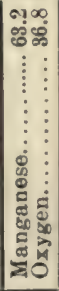 & 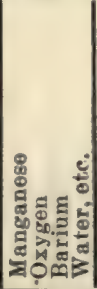 & 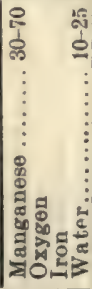 & 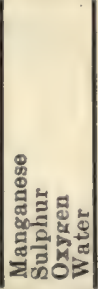 & 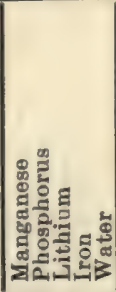 & 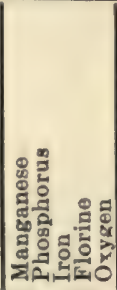 & 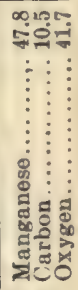 \\
\hline & 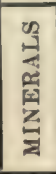 & 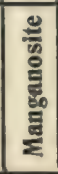 & 竎 & $\begin{array}{l}\text { 을 } \\
\frac{\mathrm{E}}{\mathrm{E}} \\
\frac{\mathrm{E}}{\mathrm{E}} \\
\text { อ }\end{array}$ & 를 & 를 & $\frac{\sum}{\frac{y}{2}}$ & 를 & 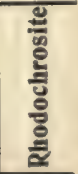 \\
\hline
\end{tabular}




\begin{tabular}{|c|c|c|c|c|c|c|c|c|c|c|}
\hline & $0^{\circ}$ & bo & & $\stackrel{+\infty}{\rightarrow-\infty}$ & $\div 0$ & 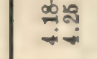 & & ن & ๓ి- & \\
\hline & 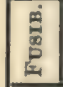 & ఏ & $\Rightarrow$ & $\Rightarrow$ & $\therefore \theta$ & bo & & 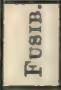 & ๑ & \\
\hline & 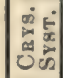 & 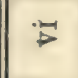 & 浔 & 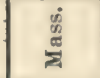 & $: \nexists$ & $: \beth$ & & 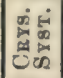 & 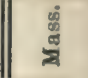 & \\
\hline$\infty$ & 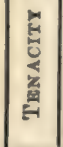 & 莺 & & 竎 & 莣 & 营 & $\frac{0}{4}$ & 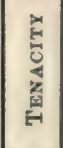 & 营 & \\
\hline $\begin{array}{l}\frac{1}{2} \\
\frac{2}{2}\end{array}$ & 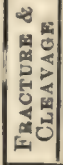 & 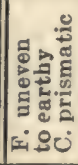 & & $\begin{array}{l}5 \\
0 \\
0 \\
\vdots \\
\vdots \\
0\end{array}$ & 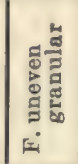 & 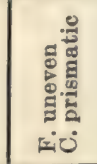 & 필 & 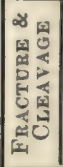 & 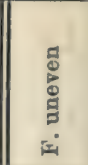 & \\
\hline$\frac{3}{2}$ & 部 & है & & 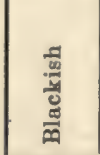 & 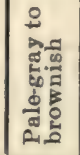 & है & $\begin{array}{l}1 \\
0 \\
0 \\
2\end{array}$ & 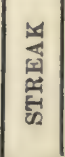 & 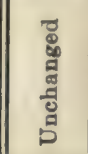 & \\
\hline$F$ & $\dot{I}$ & மले & 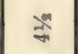 & 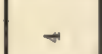 & dor & ఏेै & & 田 & 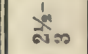 & \\
\hline $\begin{array}{l}\frac{0}{2} \\
\frac{1}{2} \\
2\end{array}$ & 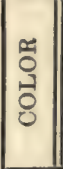 & 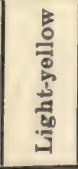 & 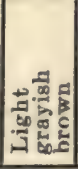 & 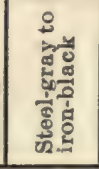 & 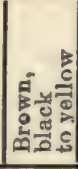 & 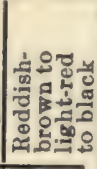 & $\begin{array}{l}4 \\
5 \\
2\end{array}$ & $\begin{array}{l}\text { 펴 } \\
0 \\
3 \\
0 \\
0\end{array}$ & 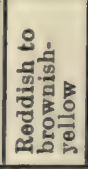 & 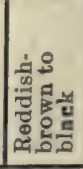 \\
\hline$\frac{5}{2}$ & 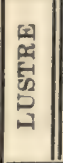 & 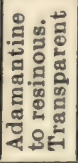 & 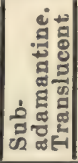 & $\begin{array}{l}\stackrel{0}{ٍ ٍ ~} \\
\frac{\pi}{2}\end{array}$ & 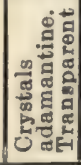 & 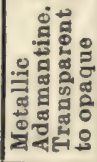 & 5 & 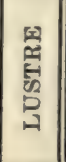 & 离 & 兽 \\
\hline 4 & 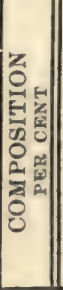 & 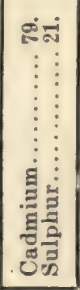 & 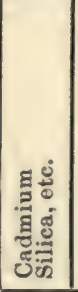 & 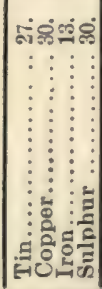 & 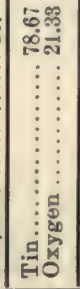 & 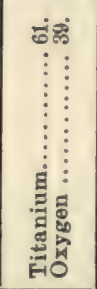 & & 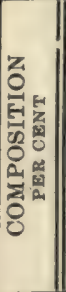 & 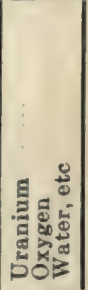 & 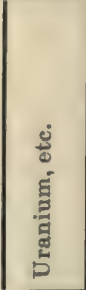 \\
\hline & 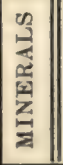 & 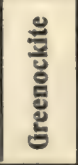 & 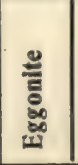 & 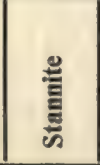 & 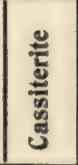 & 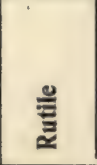 & & 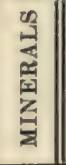 & \begin{tabular}{l|} 
总 \\
㟟
\end{tabular} & $\begin{array}{l}\text { 흘 } \\
\text { 퐆 }\end{array}$ \\
\hline
\end{tabular}


Uranium and Tungsten.

\begin{tabular}{|c|c|c|c|c|c|c|c|c|}
\hline & $\dot{\varphi}^{\circ}$ & 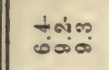 & 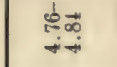 & $\begin{array}{l}\infty \rightarrow \infty \\
\infty \rightarrow \infty\end{array}$ & 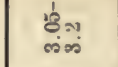 & קִ & 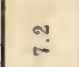 & 1. \\
\hline & 离 & $\infty$ & $\infty$ & os & $\infty$ & काले & + & $\infty_{1}$ \\
\hline อ & 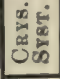 & $n$ & $=-1$ & $:=$ & : & $\therefore$ & $\therefore$ & $: \because$ \\
\hline $\begin{array}{l}j \\
z \\
z \\
z \\
0 \\
0\end{array}$ & 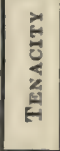 & 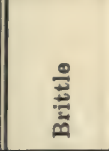 & 营 & 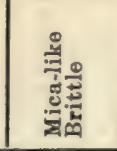 & 营 & 营 & 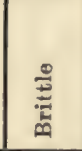 & 要 \\
\hline $\begin{array}{l}\frac{0}{3} \\
\frac{\alpha}{\alpha} \\
\frac{\alpha}{2} \\
2\end{array}$ & 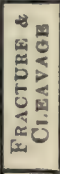 & 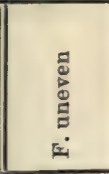 & 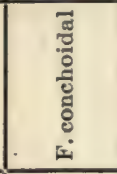 & 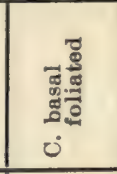 & $\begin{array}{l}\vec{J} \\
\text { J } \\
0 \\
0 \\
\end{array}$ & 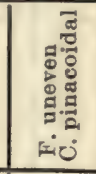 & 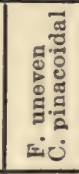 & 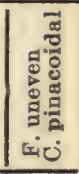 \\
\hline $\begin{array}{l}\Sigma \\
z \\
5\end{array}$ & 类 & 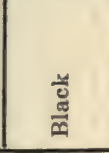 & & 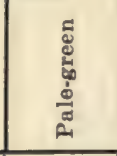 & 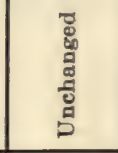 & 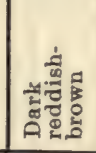 & 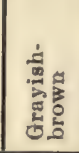 & 总 \\
\hline $\begin{array}{l}0 \\
z\end{array}$ & 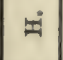 & ลี่ & 10 & ผูले & 1 & a & 6x & $\underset{+\infty}{1}$ \\
\hline $\begin{array}{l}2 \\
0 \\
2 \\
4\end{array}$ & $\begin{array}{l}0 \\
0 \\
0 \\
0 \\
8\end{array}$ & 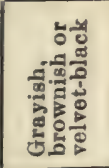 & 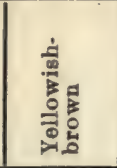 & 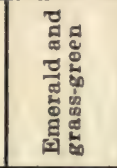 & 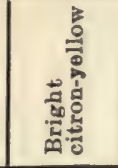 & 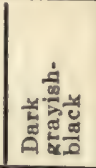 & 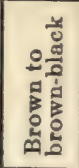 & 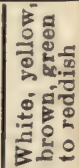 \\
\hline$\frac{5}{2}$ & 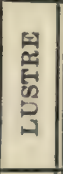 & 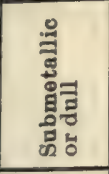 & 离 & 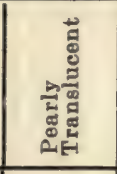 & 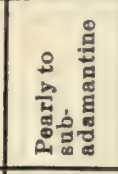 & 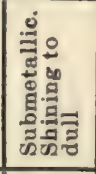 & 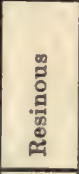 & 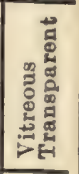 \\
\hline 7 & 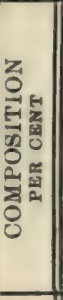 & 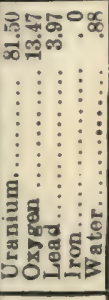 & 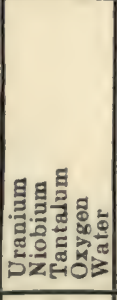 & 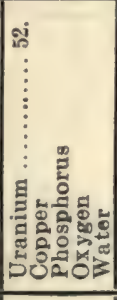 & 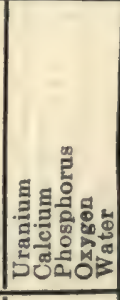 & 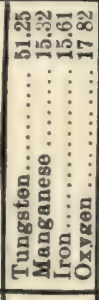 & 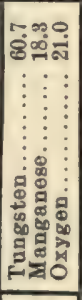 & 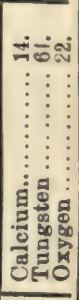 \\
\hline & 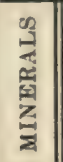 & 竎 & 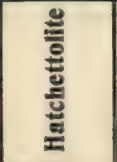 & 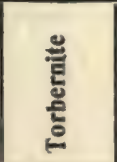 & 总 & 产 & 产 & 竎 \\
\hline
\end{tabular}


Rare Elements.

\begin{tabular}{|c|c|c|c|c|c|c|c|}
\hline \multirow{3}{*}{$\begin{array}{l}0 \\
\alpha \\
2 \\
2 \\
2 \\
2\end{array}$} & த் & $\begin{array}{l}\frac{1}{1}+\infty \\
\cos 00\end{array}$ & $\begin{array}{l}d \infty \\
\text { dis }\end{array}$ & مُ & bo & $\stackrel{9}{-}$ & $\stackrel{b}{-1}$ \\
\hline & 兽 & $\infty$ & $\underset{* \infty}{1}$ & $\infty$ & $\infty$ & • & $\bullet$ \\
\hline & 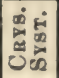 & 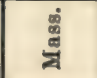 & 浔 & $:=$ & $: \Xi$ & $:=$ & :ニ \\
\hline$\frac{5}{2}$ & 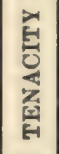 & 吾 & 营 & 营 & 营 & 营 & 苛 \\
\hline$\frac{1}{0}$ & 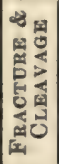 & 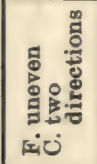 & $\begin{array}{l}\overrightarrow{3} \\
\frac{0}{0} \\
\frac{8}{0} \\
8 \\
0 \\
0\end{array}$ & 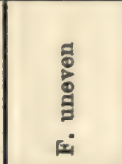 & 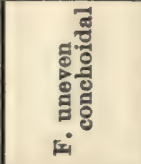 & 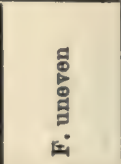 & 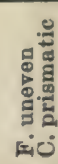 \\
\hline$\frac{1}{2}$ & 承 & & 递 & 造 & 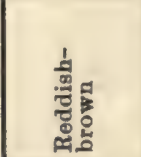 & & \\
\hline 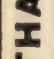 & $\dot{-1}$ & 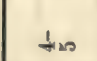 & 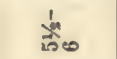 & $\frac{1}{10}$ & खें & $\infty$ & $\neq$ \\
\hline$\frac{2}{1}$ & 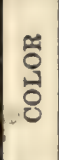 & 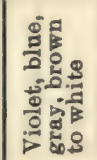 & $\begin{array}{l}\frac{y}{0} \\
\frac{3}{0} \\
\frac{1}{3} \\
\frac{8}{0} \\
\frac{8}{0}\end{array}$ & 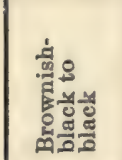 & 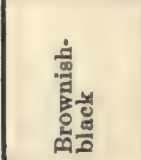 & 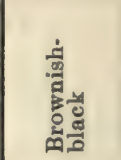 & 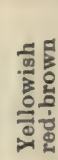 \\
\hline$\frac{2}{n}$ & 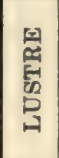 & 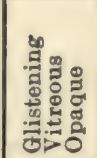 & 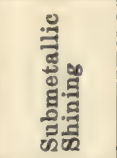 & 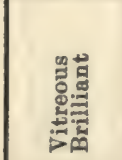 & 总 & 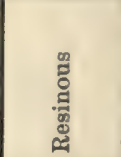 & 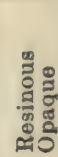 \\
\hline $\begin{array}{l}\frac{2}{0} \\
\frac{2}{2} \\
5 \\
5\end{array}$ & 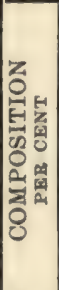 & 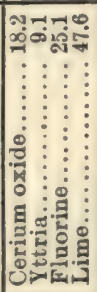 & 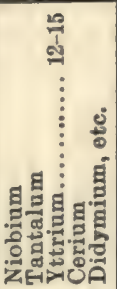 & 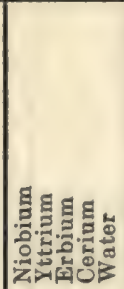 & 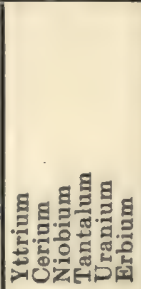 & 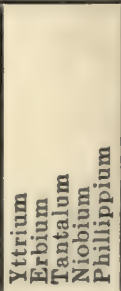 & 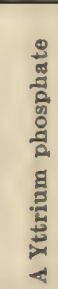 \\
\hline$\frac{x}{0}$ & 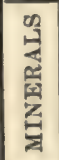 & 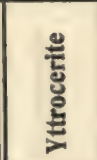 & 竞 & 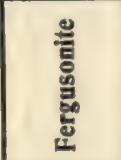 & 兘 & $\frac{\stackrel{3}{\frac{E}{n}}}{\frac{5}{n}}$ & 兽 \\
\hline
\end{tabular}


Rare Elements-Aluminium.

\begin{tabular}{|c|c|c|c|c|c|c|c|c|c|}
\hline$i^{2}$ & ๑ & $\begin{array}{l}\text { \$口 } \\
\text { Tin }\end{array}$ & $\begin{array}{l}\infty \\
+\infty \\
+\infty\end{array}$ & & 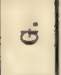 & $\stackrel{d}{+\infty}$ & $\stackrel{\oplus}{=}$ & 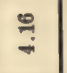 & 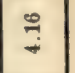 \\
\hline $\begin{array}{l}0 \\
0 \\
0\end{array}$ & 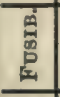 & $\infty$ & $\infty$ & & 䓢 & $\infty$ & $\infty$ & • & 0 \\
\hline$\underset{\mathbf{w}}{\mathbf{\alpha}}$ & 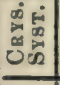 & 泍 & $\geq$ & & 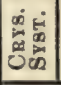 & 岀国 & $\vec{\nabla} \approx$ & 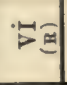 & "10영 \\
\hline$\frac{z}{z}$ & 廌 & 竎 & 营 & & 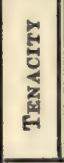 & 馬 & & & \\
\hline $\begin{array}{l}\frac{2}{\Sigma} \\
\frac{2}{0}\end{array}$ & 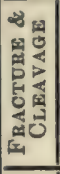 & 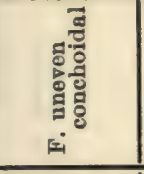 & 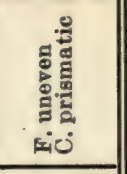 & & 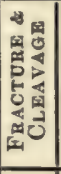 & 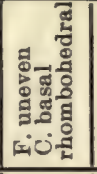 & & & \\
\hline $\begin{array}{l}0 \\
\frac{2}{4} \\
\Sigma\end{array}$ & 类 & & & $\begin{array}{l}a \\
a \\
2\end{array}$ & 商 & 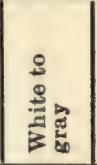 & & & \\
\hline$\frac{3}{2}$ & 田 & $\infty$ & bio & $\begin{array}{l}5 \\
5\end{array}$ & $\dot{I}$ & $\infty$ & $\infty$ & $\infty$ & 0 \\
\hline $\begin{array}{l}I \\
z \\
\\
\end{array}$ & 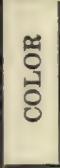 & 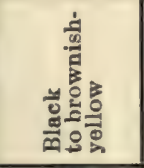 & 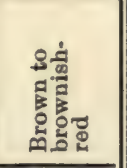 & $\frac{\frac{5}{2}}{\frac{2}{2}}$ & $\begin{array}{l}\text { 유 } \\
\text { 잉 }\end{array}$ & 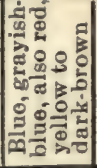 & 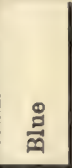 & శึ & 产 \\
\hline$\frac{2}{2}$ & 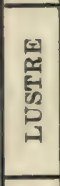 & 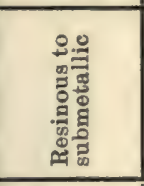 & 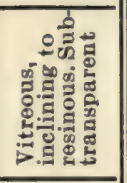 & $\frac{1}{4}$ & 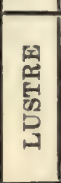 & 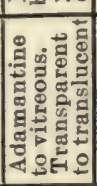 & 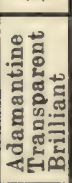 & 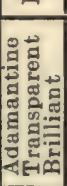 & 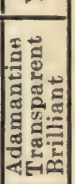 \\
\hline$\frac{5}{2}$ & 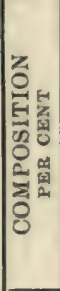 & 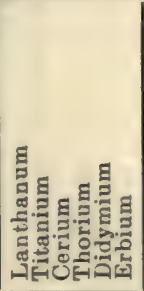 & 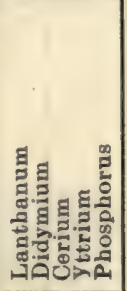 & & 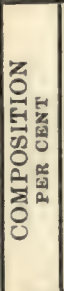 & 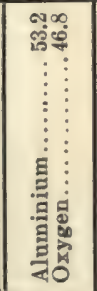 & 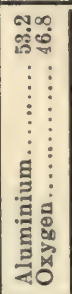 & 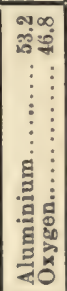 & 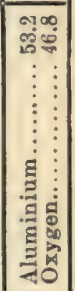 \\
\hline$\frac{\frac{5}{20}}{\frac{u}{0}}$ & 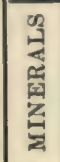 & 흘 & 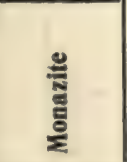 & & 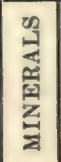 & 틀 & 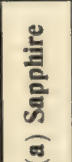 & 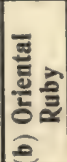 & 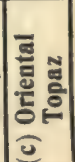 \\
\hline
\end{tabular}


Aluminium.

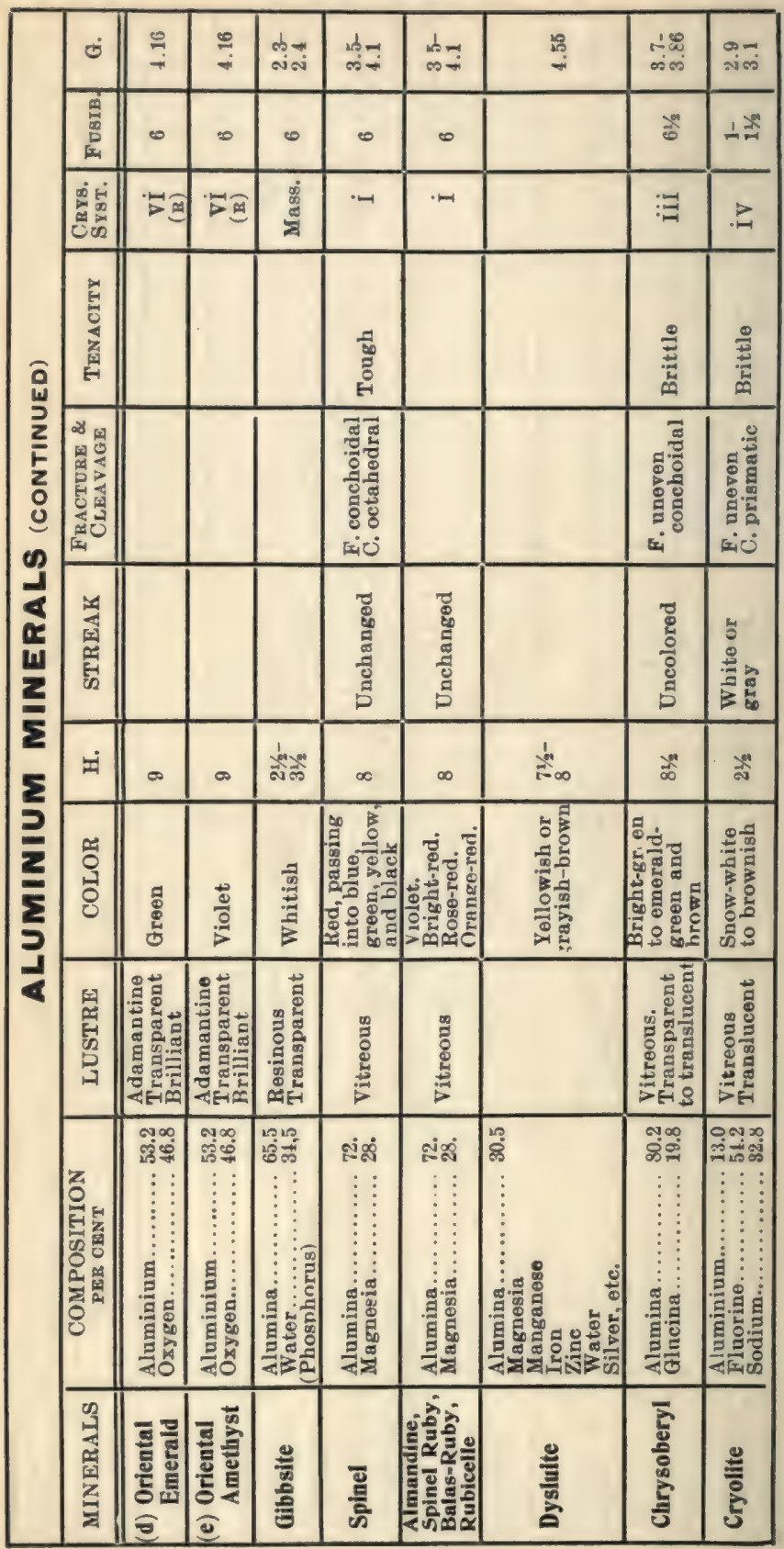


Aluminium.

\begin{tabular}{|c|c|c|c|c|c|c|c|c|}
\hline & க் & $\overbrace{-\infty}^{\infty}$ & $\begin{array}{l}\text { कb? } \\
\text { sing } \\
\text { ninis }\end{array}$ & $\begin{array}{l}d= \\
\sin \theta\end{array}$ & $\begin{array}{l}5 \\
\text { o. } \\
\text { s. }\end{array}$ & $\begin{array}{l}b_{\infty} \\
\text { ஸisi.j }\end{array}$ & 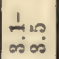 & $\begin{array}{l}\text { के } \vec{~} \\
\text { जis }\end{array}$ \\
\hline & 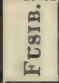 & 0 & 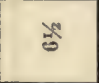 & $\infty$ & $\bullet$ & 0 & - & $\infty$ \\
\hline & 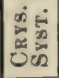 & $>$ & Гळ & $>$ & 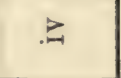 & $\begin{array}{l}\infty \\
\stackrel{\infty}{\infty} \\
\stackrel{\infty}{\Xi}\end{array}$ & $\vdots \Xi$ & $: \Xi$ \\
\hline 으 & 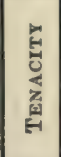 & 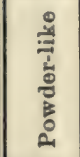 & 岦 & 莣 & 莣 & 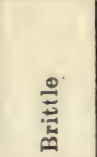 & 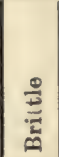 & 䒿 \\
\hline $\begin{array}{l}2 \\
z \\
z \\
2 \\
0 \\
0\end{array}$ & 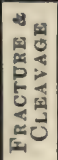 & 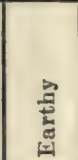 & 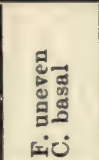 & 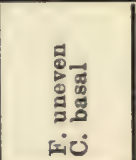 & 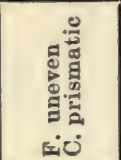 & 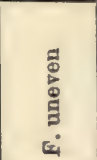 & 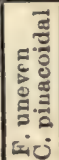 & 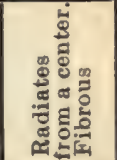 \\
\hline$\frac{1}{a}$ & 茨 & $\frac{8}{3}$ & 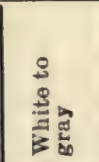 & 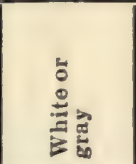 & 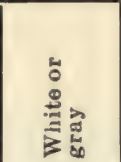 & 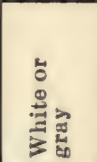 & & 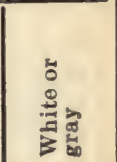 \\
\hline 2 & $\dot{A}$ & $\underbrace{\prime}$ & $*$ & 0 & 4 bo & 0 & 10 & हैं \\
\hline$\frac{2}{2}$ & $\begin{array}{l}\text { 유 } \\
\text { ○ } \\
\text { ᄋ }\end{array}$ & $\stackrel{8}{3}$ & 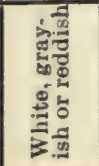 & 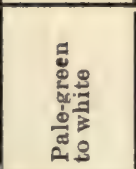 & $\frac{\stackrel{2}{3}}{\frac{3}{8}}$ & 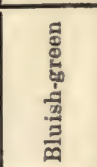 & 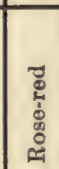 & 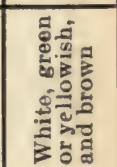 \\
\hline$\alpha$ & 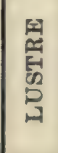 & 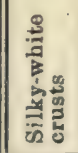 & 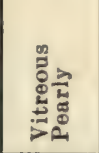 & 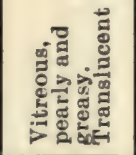 & 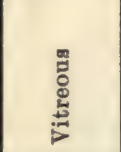 & है & 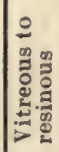 & 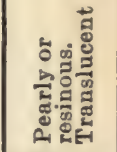 \\
\hline & 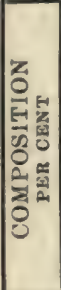 & 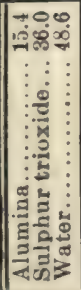 & 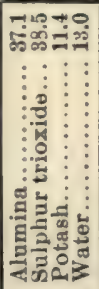 & 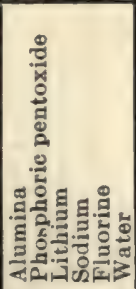 & 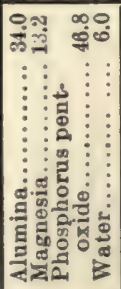 & 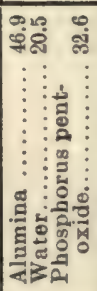 & 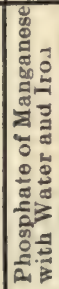 & 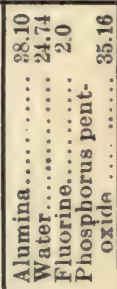 \\
\hline & 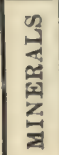 & $\begin{array}{l}\text { 평 } \\
\text { 응 } \\
\text { 틀 }\end{array}$ & 尊 & $\begin{array}{l}\text { 를 } \\
\text { 을 } \\
\text { 를 }\end{array}$ & 总 & $\begin{array}{l}\text { 을 } \\
\text { 를 }\end{array}$ & 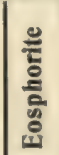 & 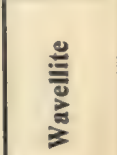 \\
\hline
\end{tabular}


Magnesium-Calcium.

\begin{tabular}{|c|c|c|c|c|c|c|c|c|c|c|}
\hline ச் & $\ddot{b}$ & 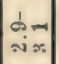 & 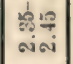 & $\begin{array}{l}\dot{d} x \\
\operatorname{lin}^{\circ}\end{array}$ & $=$ & s. & & $0^{\circ}$ & 趈 & $\begin{array}{l}\text { ळొ } \\
\text { ๙ే }\end{array}$ \\
\hline 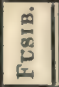 & $\infty$ & $-1=0$ & $\infty$ & $\infty$ & $\Rightarrow$ & $\infty$ & & 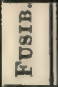 & - & के \\
\hline 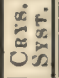 & $-m$ & $: \cong$ & 5 영 & $\cdot \vec{D} \widehat{\Xi}$ & $: \Xi$ & $\cdot$ & & 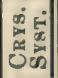 & . w & D \\
\hline 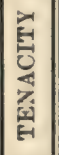 & 咅 & 乵 & 营 & 营 & 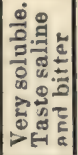 & 莣 & & 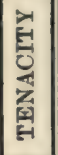 & 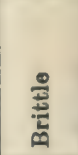 & 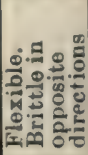 \\
\hline 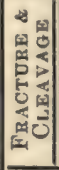 & 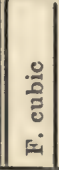 & 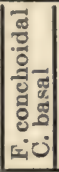 & $\begin{array}{l}\text { चี } \\
\text { ळ } \\
0 \\
0\end{array}$ & 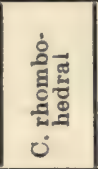 & 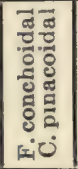 & 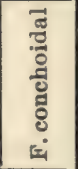 & & 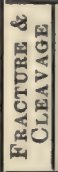 & 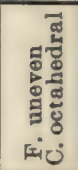 & 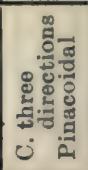 \\
\hline 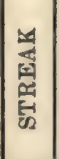 & 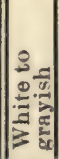 & $\frac{9}{3}$ & 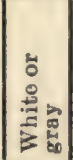 & 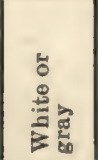 & $\frac{8}{3}$ & 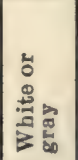 & $\begin{array}{l}0 \\
\alpha \\
0 \\
11\end{array}$ & 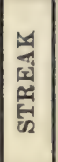 & 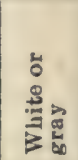 & 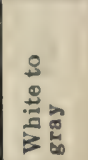 \\
\hline 琏 & : & תמ & సે & $\omega^{2 x}$ & ล่ลึ่ & $\sum^{\infty}$ & $\overline{5}$ & | & + & $\approx \infty$, \\
\hline \begin{tabular}{c}
1 \\
\multirow{2}{*}{} \\
0 \\
0 \\
0 \\
0
\end{tabular} & 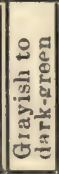 & : & 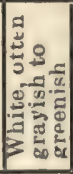 & 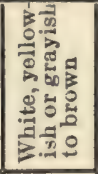 & 总 & 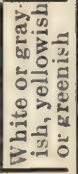 & $\frac{5}{3}$ & $\begin{array}{l}\text { 땡 } \\
8 \\
0 \\
8\end{array}$ & 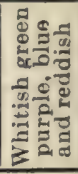 & 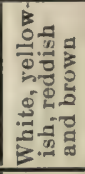 \\
\hline 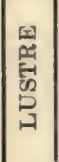 & 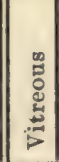 & 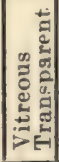 & 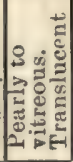 & 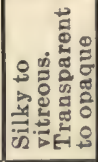 & 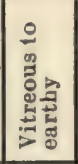 & 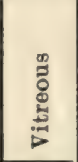 & & 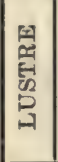 & 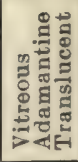 & 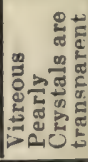 \\
\hline 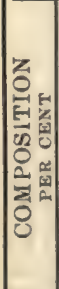 & 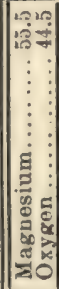 & 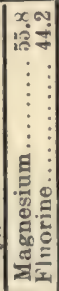 & 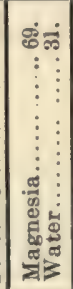 & 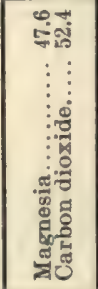 & 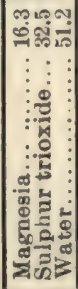 & 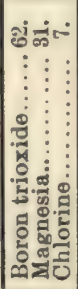 & & 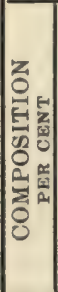 & 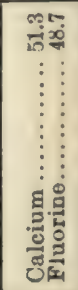 & 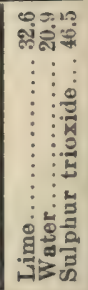 \\
\hline 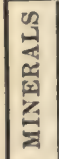 & 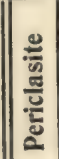 & 흘 & 冚 & 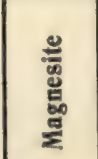 & $\begin{array}{l}\text { 플 } \\
\text { 모 } \\
\text { 또 }\end{array}$ & ڤ્ّ & & 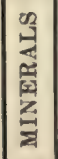 & 흘 & 톯 \\
\hline
\end{tabular}


Calclum.

\begin{tabular}{|c|c|c|c|c|c|c|c|c|c|}
\hline & $\dot{0}$ & 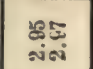 & $\stackrel{18}{-3}$ & ஸे & 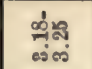 & $\begin{array}{l}\frac{1}{1}+\infty \\
\text { जisi }\end{array}$ & & $\begin{array}{l}\mathscr{W} \\
\text { a } \\
\text { i }\end{array}$ & $\begin{array}{l}\text { bo } \\
\text { isi }\end{array}$ \\
\hline & $\begin{array}{l}\text { 量 } \\
\text { 品 }\end{array}$ & का & $\gtrsim$ & $\stackrel{x}{\Rightarrow}$ & का & 0 & $\Rightarrow$ & 0 & 0 \\
\hline & 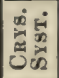 & ב ב & & $i=1$ & 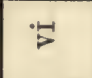 & $5 \mathscr{B}$ & 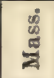 & $: \sqsupseteq$ & $5 \mathscr{B}$ \\
\hline 0 & 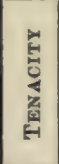 & 营 & 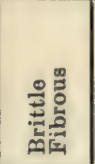 & 营 & 营 & 营 & 营 & 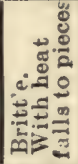 & 焉 \\
\hline $\begin{array}{l}\frac{1}{2} \\
\frac{2}{5} \\
2 \\
0 \\
0\end{array}$ & 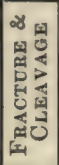 & 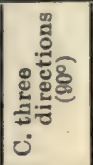 & हैं & 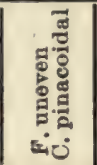 & 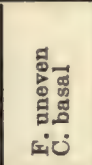 & 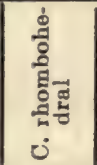 & $\stackrel{\text { ह }}{E}$ & 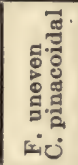 & 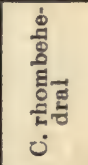 \\
\hline $\begin{array}{l}0 \\
1 \\
\alpha \\
0 \\
2\end{array}$ & 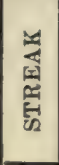 & 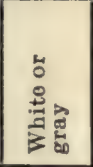 & $\stackrel{8}{d}$ & 蛋 & 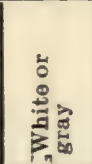 & 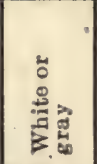 & 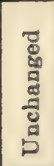 & 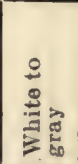 & 告 \\
\hline$\overline{5}$ & व & d & $\Rightarrow$ & $+\infty x^{*}$ & 10 & $\infty$ & $\begin{array}{l}200 \\
0 \\
0\end{array}$ & ले & $\frac{1}{a b}$ \\
\hline$\frac{5}{3}$ & \begin{tabular}{l}
\multirow{2}{0}{} \\
0 \\
0 \\
0
\end{tabular} & 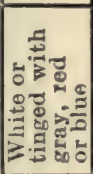 & 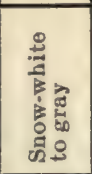 & 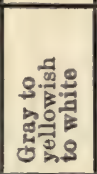 & 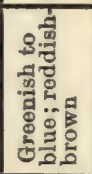 & 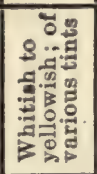 & 总 & 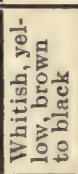 & 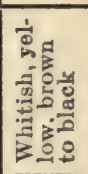 \\
\hline & 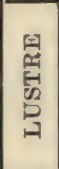 & 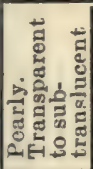 & 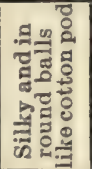 & 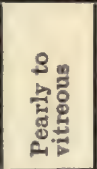 & 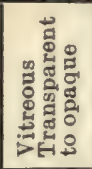 & 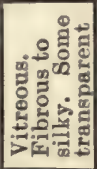 & 㓂 & 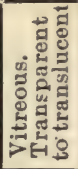 & 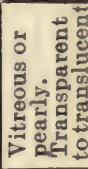 \\
\hline & 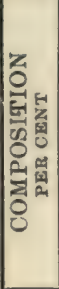 & 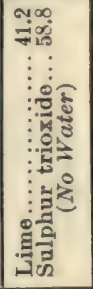 & 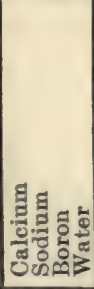 & 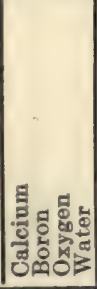 & 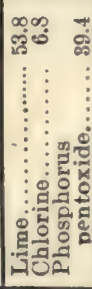 & 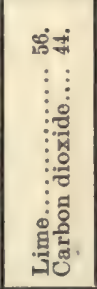 & 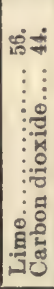 & 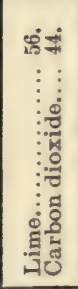 & 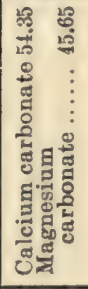 \\
\hline & 量 & 总 & 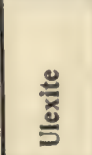 & 总 & 苛 & 总 & 䬧 & 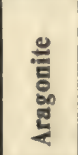 & 플 \\
\hline
\end{tabular}


Barium, Strontium, Etc.

\begin{tabular}{|c|c|c|c|c|c|c|c|}
\hline$\infty$ & $0^{\circ}$ & $\stackrel{d r}{+}=$ & d্d:? & $\underset{\infty i-i}{d}$ & ஸ่ & $d_{-i n i}^{d}$ & มี \\
\hline$\underset{1}{\alpha}$ & $\begin{array}{l}\dot{\varphi} \\
\text { 总 } \\
\text { 品 }\end{array}$ & $*$ & 埚 & $\underset{\infty}{x \rightarrow}$ & - & $\approx$ & $\gtrsim$ \\
\hline 2 & 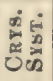 & $: \Xi$ & $:=$ & $: \Xi$ & 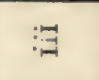 & 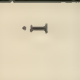 & $\cdot \rightarrow$ \\
\hline$\frac{5}{2}$ & 总 & 敔 & 营 & 莣 & $\overbrace{}^{\circ}$ & 莣 & 咅 \\
\hline$\frac{5}{5}$ & 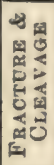 & 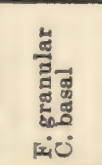 & 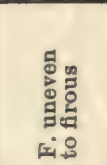 & 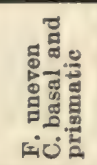 & 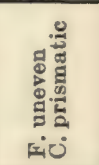 & 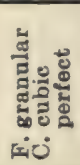 & $\frac{0}{0}$ \\
\hline$\frac{4}{2}$ & 霆 & $\begin{array}{l}5 \\
\stackrel{8}{8} \\
3 \\
3\end{array}$ & 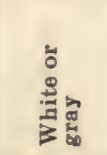 & 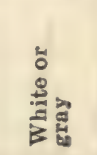 & 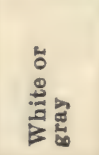 & 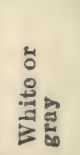 & 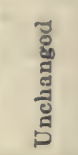 \\
\hline 0 & व & $\omega_{\infty}^{\infty}$ & $d \vec{b}$ & ம் & लैं & $\infty$ & NO \\
\hline$\frac{5}{5}$ & 융 & 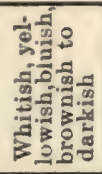 & 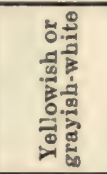 & 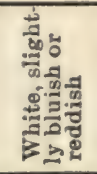 & 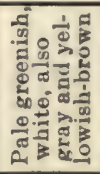 & 营 & 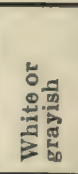 \\
\hline $\begin{array}{l}0 \\
0 \\
2\end{array}$ & 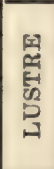 & 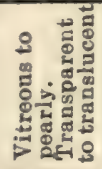 & 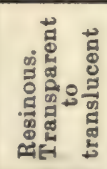 & 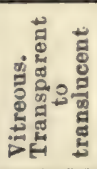 & 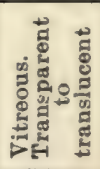 & 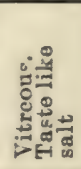 & 总 \\
\hline $\begin{array}{l}2 \\
1 \\
2 \\
0 \\
0 \\
0 \\
5 \\
5\end{array}$ & 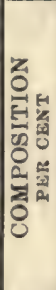 & 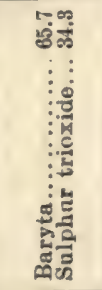 & 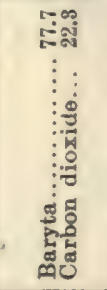 & 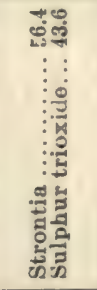 & 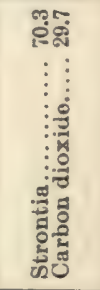 & 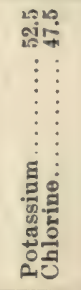 & 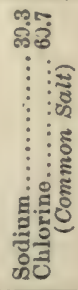 \\
\hline$\frac{a}{a}$ & 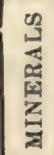 & है & 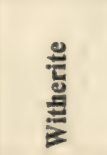 & $\frac{3}{3}$ & 音 & 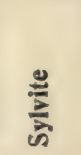 & 플 \\
\hline
\end{tabular}


Barium, Strontium, Etc.

\begin{tabular}{|c|c|c|c|c|c|c|c|}
\hline ż & ن் & $\stackrel{9}{-1}$ & 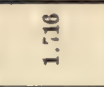 & 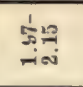 & & +2 & 活 \\
\hline क & 兽 & $\Rightarrow$ & 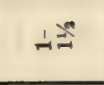 & -1 & & - & -10 \\
\hline 뜬 & 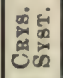 & : & . & $: \Xi$ & "HË & Z & $\cdot x$ \\
\hline $\begin{array}{l}\frac{5}{2} \\
5\end{array}$ & 荡 & 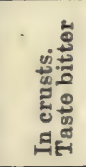 & 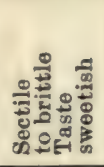 & 莣 & 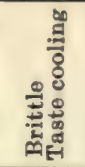 & 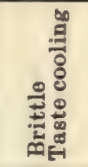 & 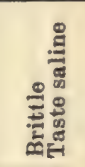 \\
\hline $\begin{array}{l}\frac{2}{0} \\
\frac{2}{2} \\
\frac{2}{4}\end{array}$ & 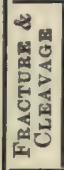 & 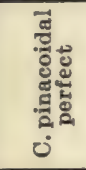 & 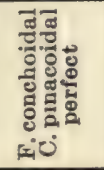 & 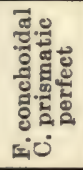 & 㱟 & 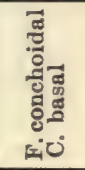 & $\begin{array}{l}\overrightarrow{5} \\
\stackrel{0}{0} \\
\frac{0}{0} \\
0 \\
0 \\
0\end{array}$ \\
\hline $\begin{array}{l}0 \\
2 \\
2 \\
2\end{array}$ & 承 & 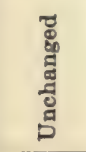 & 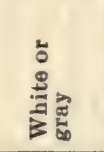 & $\stackrel{8}{\sharp}$ & 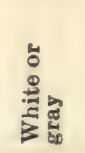 & હ્ટ્心 & 承 \\
\hline$=$ & चं & $\sum_{-\infty}^{\infty}$ & ลสั & N & $\Rightarrow$ & $1 \geq$ & $\sum_{-\infty}^{\prime}$ \\
\hline$\frac{\infty}{3}$ & 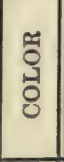 & 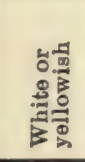 & $\frac{8}{3}$ & 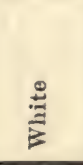 & 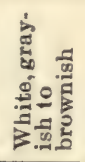 & 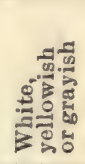 & 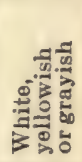 \\
\hline $\begin{array}{l}0 \\
5 \\
1 \\
0 \\
0 \\
-\end{array}$ & 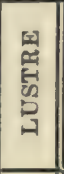 & 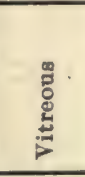 & 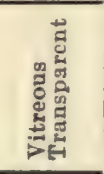 & $\stackrel{\vec{E}}{\mathrm{~L}}$ & 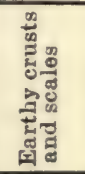 & 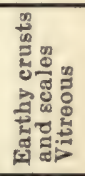 & 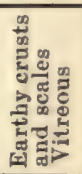 \\
\hline $\begin{array}{l}E \\
2 \\
E \\
2 \\
0 \\
a \\
\infty \\
=\end{array}$ & 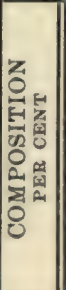 & 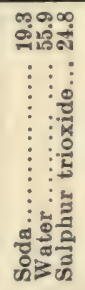 & 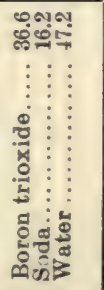 & 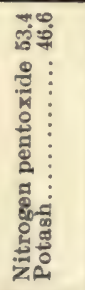 & 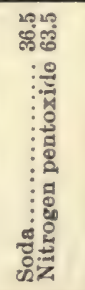 & 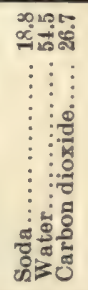 & 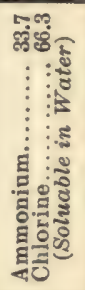 \\
\hline$\frac{2}{a}$ & 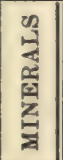 & 흘 & 릉 & $\frac{g}{z}$ & 를 & 톨 & 咅 \\
\hline
\end{tabular}


Acid Minerals.

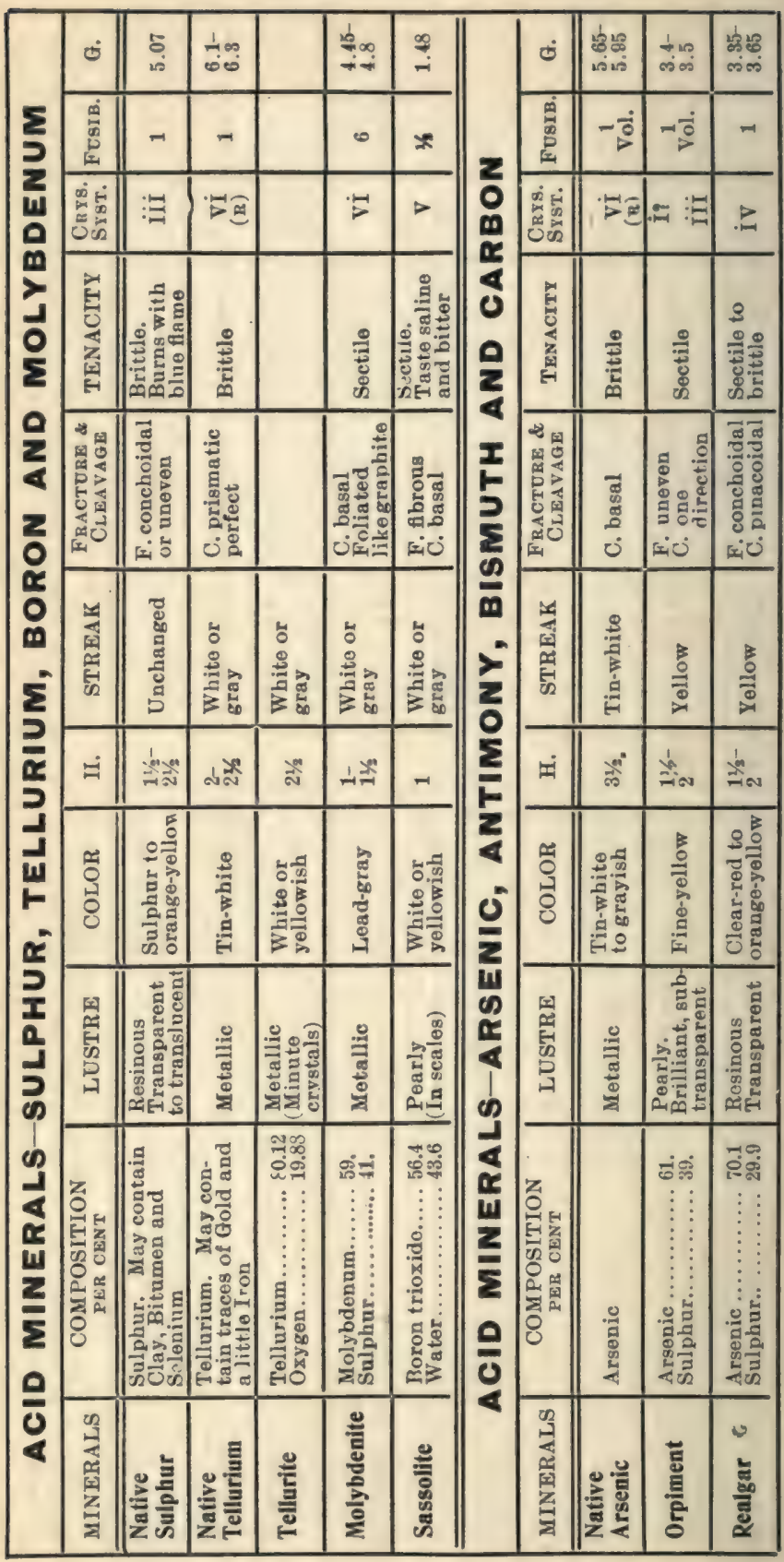


Acid Minerals.

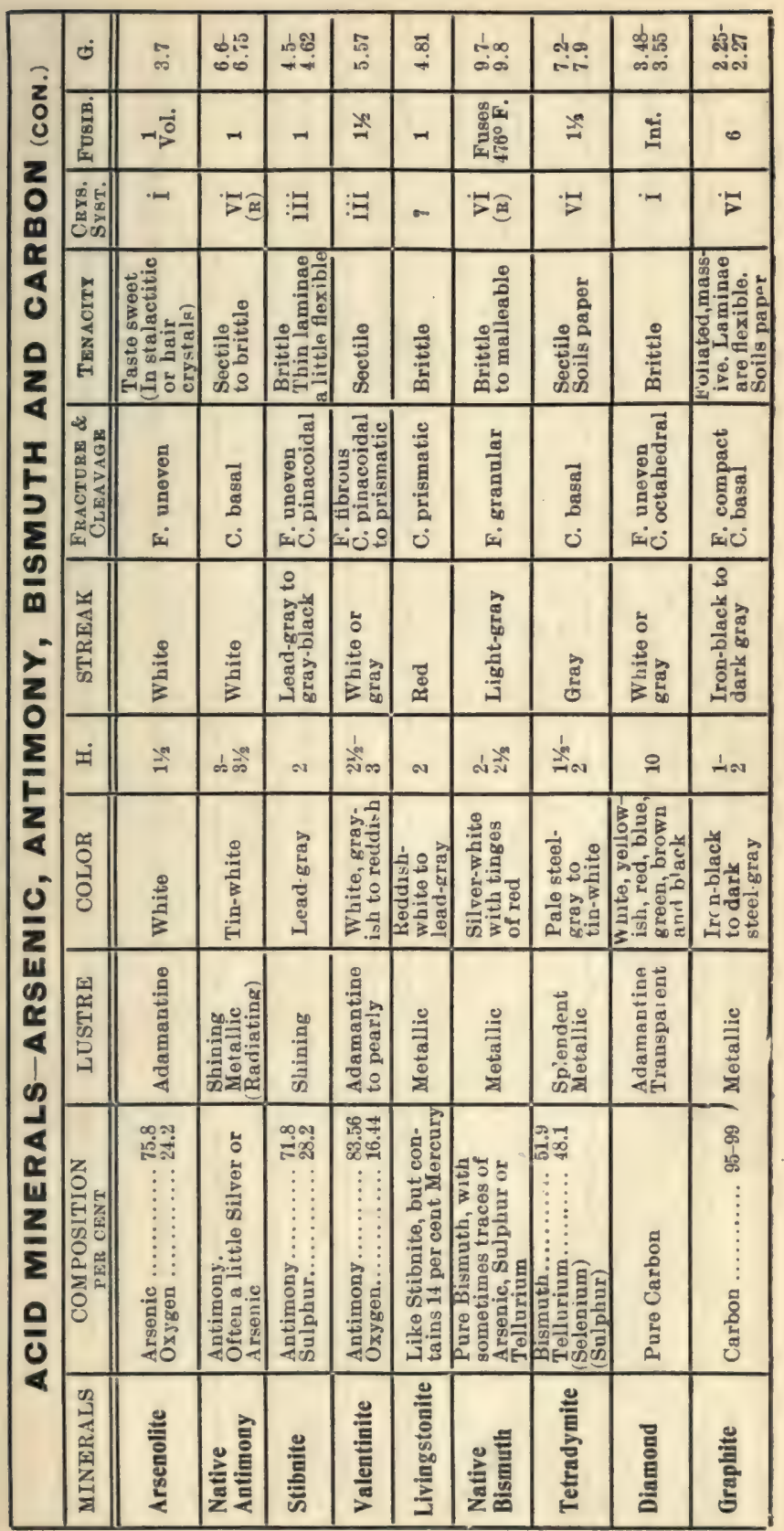


Hydrocarbons-Coal.

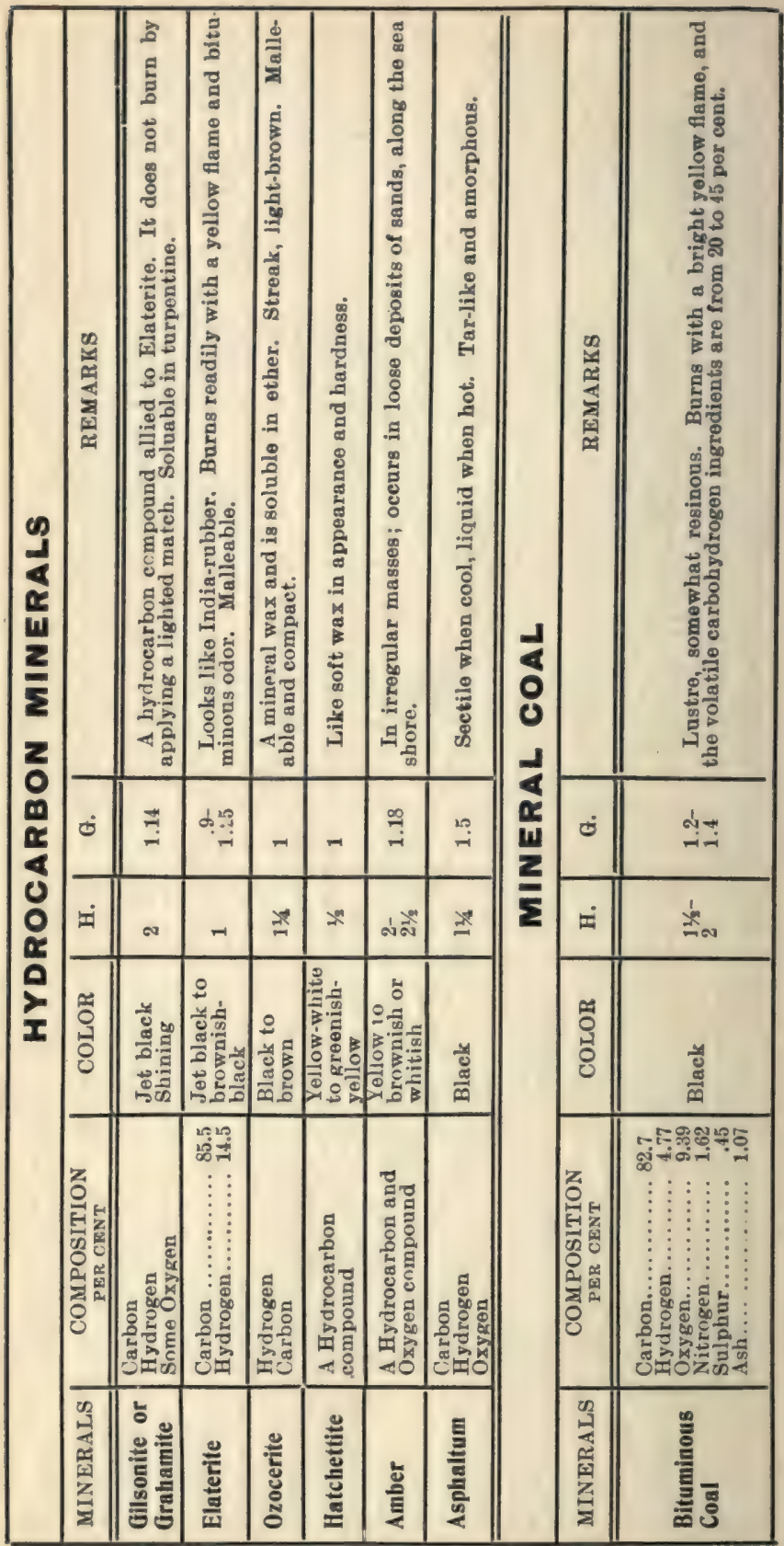


Coal.

\begin{tabular}{|c|c|c|c|c|c|c|c|}
\hline 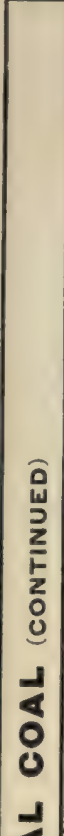 & 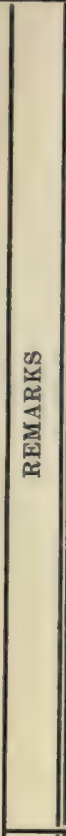 & 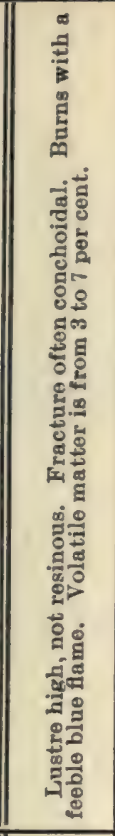 & 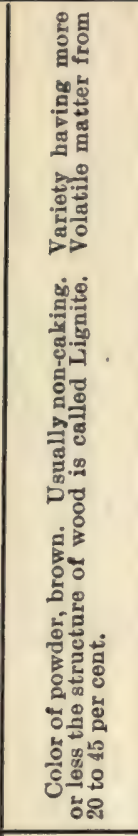 & 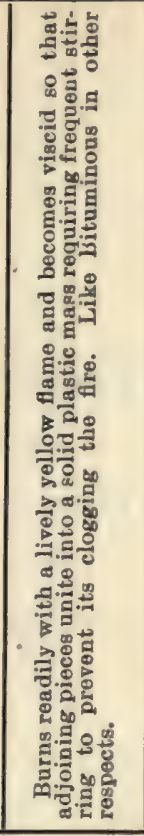 & 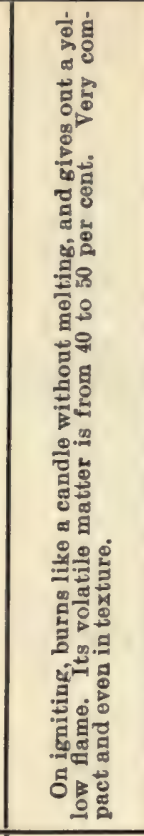 & 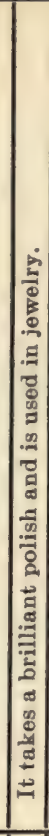 & 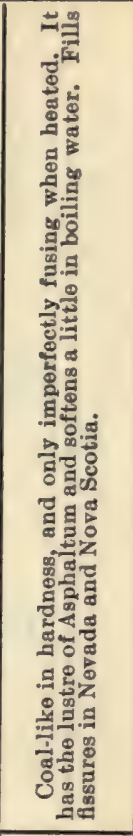 \\
\hline$\frac{a}{2}$ & نه & 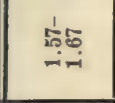 & $\stackrel{+}{ت}$ & & 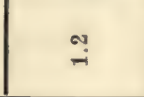 & & 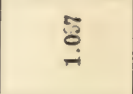 \\
\hline 5 & 퍼 & ่ลั & बें & & ه & $\infty$ & $\perp \infty$ \\
\hline & 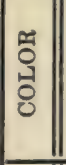 & $\begin{array}{l}\text { है } \\
\text { लّ }\end{array}$ & 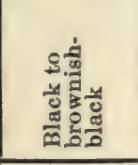 & $\begin{array}{l}\text { है } \\
\text { है }\end{array}$ & 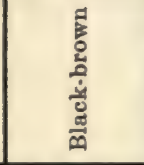 & 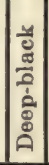 & 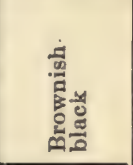 \\
\hline & 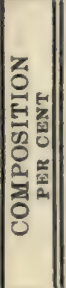 & 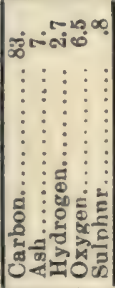 & 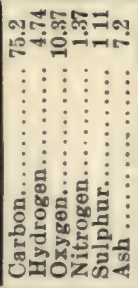 & 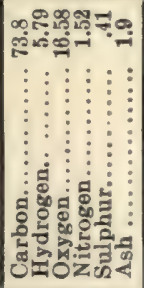 & 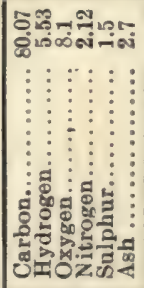 & 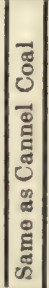 & 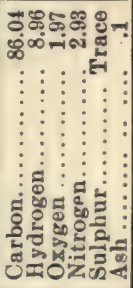 \\
\hline & 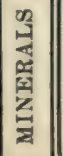 & 总 & $\begin{array}{l}\bar{E} \\
\text { ह } \\
\text { है } \\
\text { है }\end{array}$ & 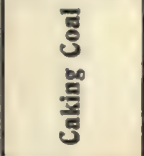 & 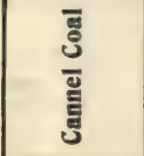 & 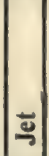 & 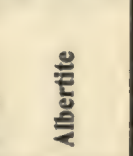 \\
\hline
\end{tabular}




\section{Analytical Tables of OMinerals}

\section{DIVISION II}

\section{MINERALS FORMING ROCKS}

\section{Silica and the Silicates \\ Lustre, Unmetallic \\ Streak, White or Gray}

The terrane's or earth's crust is made out of different kind of rocks, and these rocks are made out of the various minerals met with in nature. Rocks are distinguished from each other by the kind of minerals forming them; it is, therefore, necessary that we be able to identify these minerals in order to make the proper classification of rock formations.

In the following division only the silicon minerals, formings rocks, are described; these include also many varieties possessing considerable commercial value, and when occurring in workable quantities they might be termed "ores." The minerals of calcium and aluminum are important minerals of the rock forming kind, but they are here included under Division I, with the ores. 


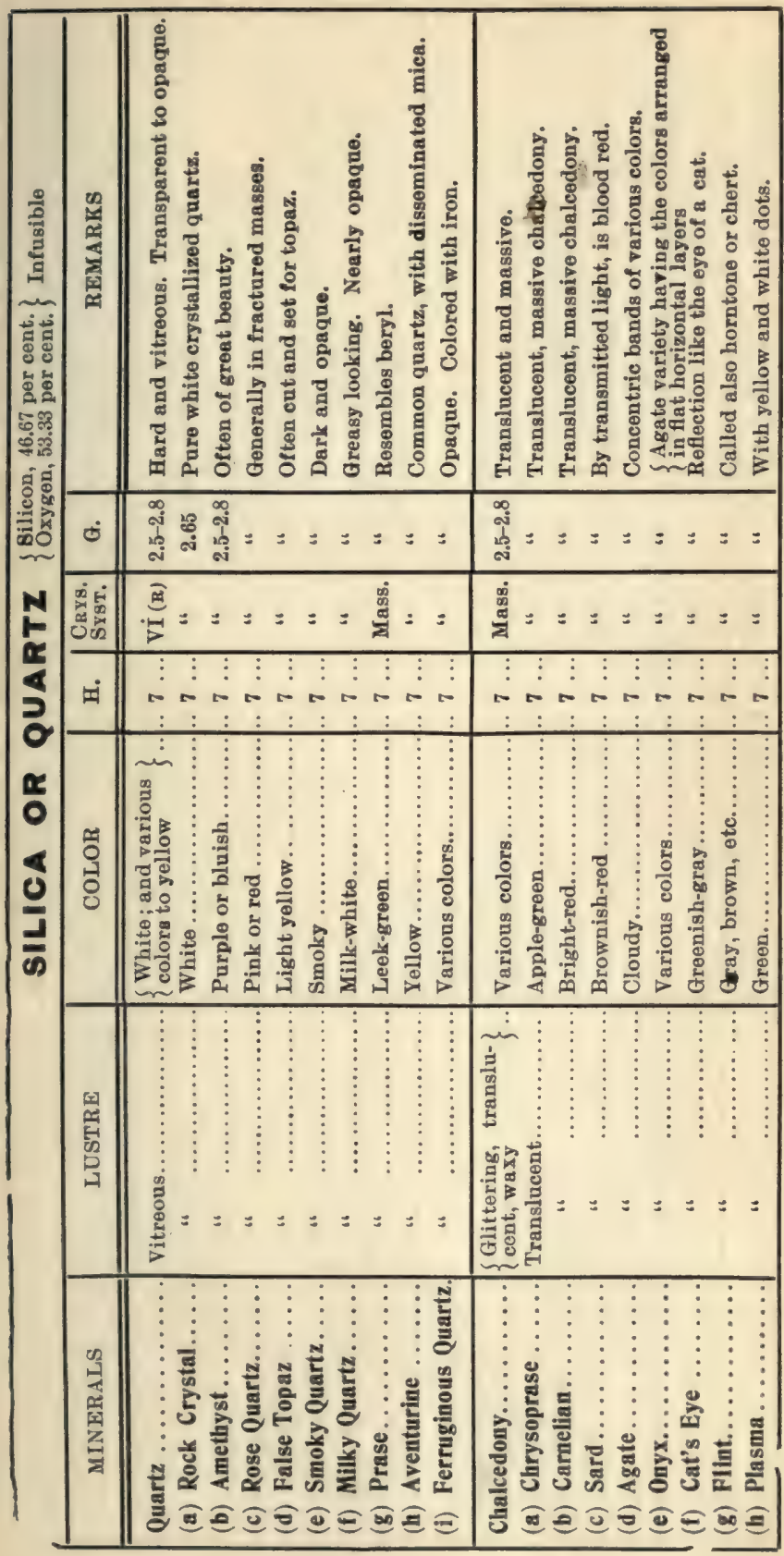




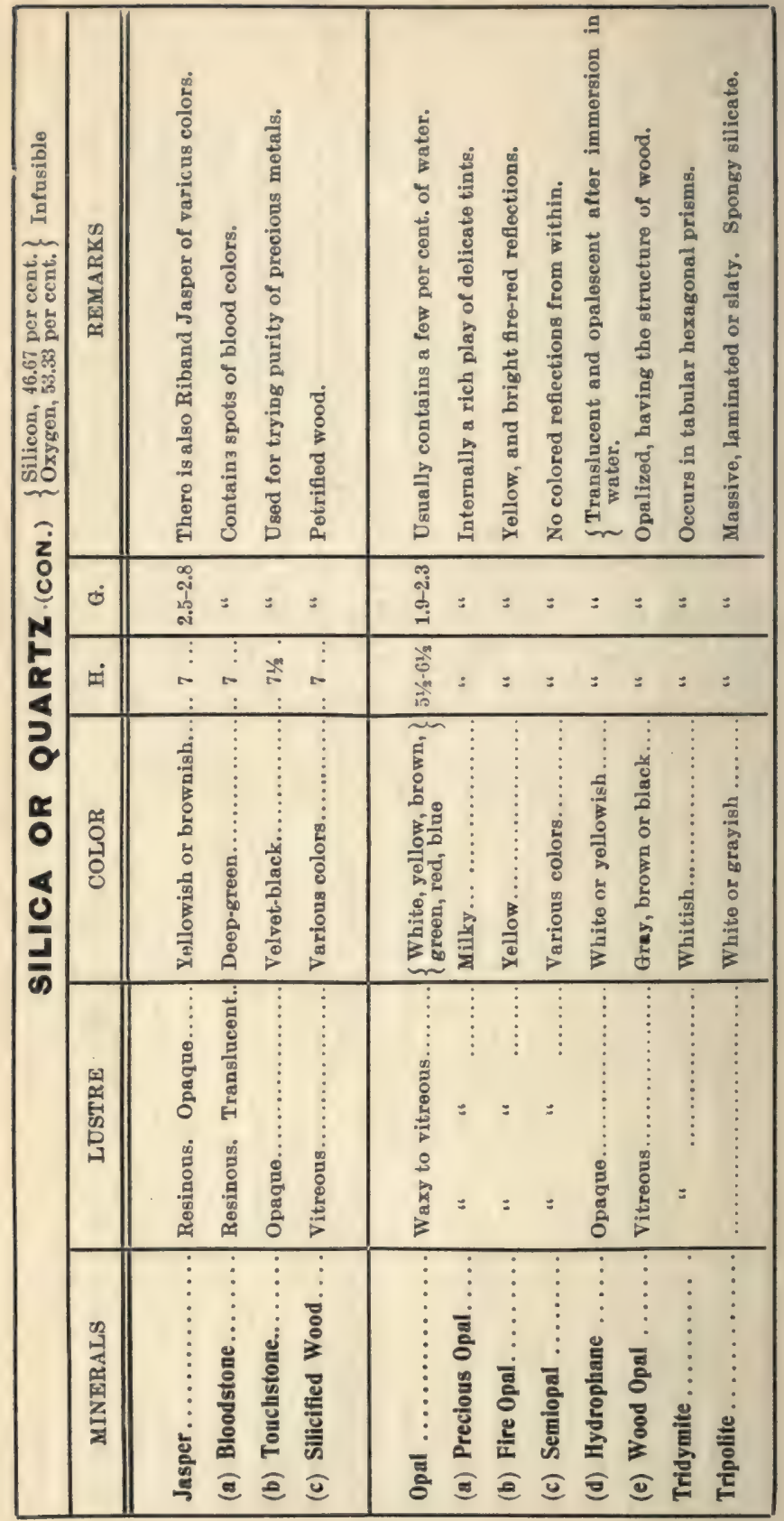


Bisilicates.

\begin{tabular}{|c|c|c|c|c|c|c|c|}
\hline & ல் & \begin{tabular}{l}
$\frac{1}{\infty} \infty$ \\
\hdashline 00
\end{tabular} & 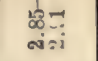 & $\begin{array}{l}1120 \\
\text { ase }\end{array}$ & $\frac{1}{\sin \theta}$ & 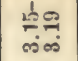 & $\begin{array}{l}1 \\
60 ? \\
\text { aici }\end{array}$ \\
\hline & 罢 & का & + & + & ल हैं & है & मूने \\
\hline & 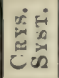 & : & $Z$ & . & $D$ & $D$ & $\stackrel{1}{>}$ \\
\hline & 突 & 䒯 & 菢 & 站 & 营 & 䓫 & 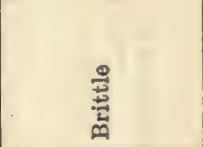 \\
\hline & 象 & 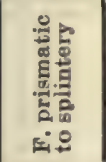 & 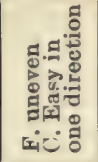 & 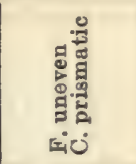 & 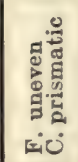 & 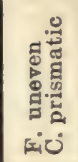 & 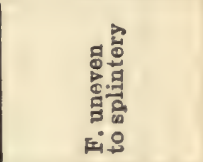 \\
\hline $\begin{array}{l}0 \\
\text { uI }\end{array}$ & 茎 & 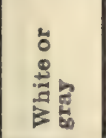 & 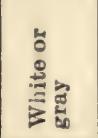 & 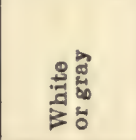 & 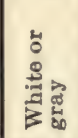 & 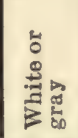 & 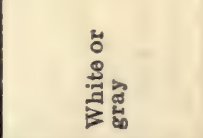 \\
\hline 0 & $\ddot{\nexists}$ & ते & 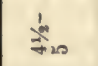 & ه内א & 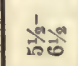 & के & $\stackrel{1}{1}=\infty$ \\
\hline$\frac{1}{6}$ & $\begin{array}{l}\text { 떠 } \\
0 \\
0 \\
0 \\
0\end{array}$ & 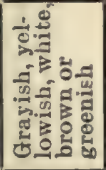 & 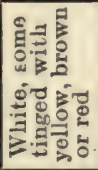 & 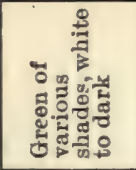 & 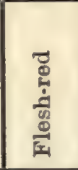 & 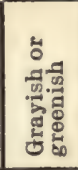 & 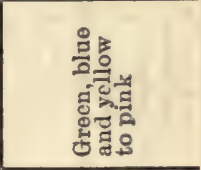 \\
\hline & 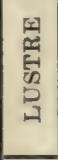 & 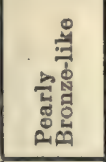 & 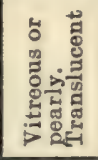 & 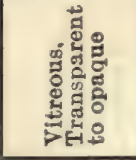 & 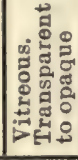 & 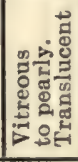 & 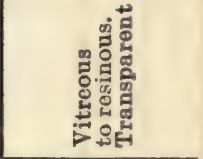 \\
\hline & 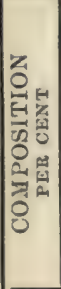 & 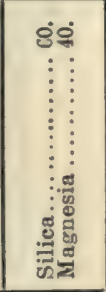 & 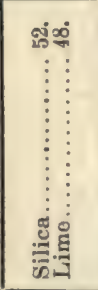 & 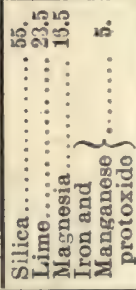 & 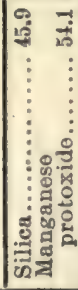 & 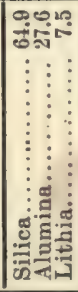 & 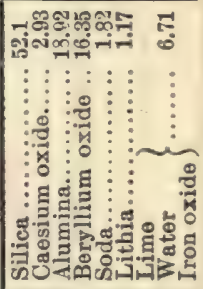 \\
\hline & 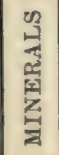 & 总 & $\begin{array}{l}\text { 플 } \\
\text { 봄 } \\
\text { 흥 }\end{array}$ & है & $\begin{array}{l}\text { 를 } \\
\text { 를 } \\
\text { 를 }\end{array}$ & 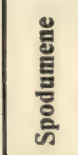 & $\overline{\mathrm{D}^{\circ}}$ \\
\hline
\end{tabular}


Bisillcates.

\begin{tabular}{|c|c|c|c|c|c|c|c|}
\hline & ه & $\stackrel{4}{\mathrm{c}}$ & $\begin{array}{l}\text { d. } \\
\text { ive }\end{array}$ & $\begin{array}{l}d \\
\text { aim }\end{array}$ & कि को & âे & $\begin{array}{l}\text { Nyen } \\
\text { } \operatorname{sen}\end{array}$ \\
\hline & 产 & + & $\dot{c}+$ & + & + & 岕 & $\omega+$ \\
\hline & 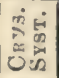 & . & . & $Z$ & : & 浔 &. \pm \\
\hline & 省 & 营 & 竞 & 营 & 壳 & 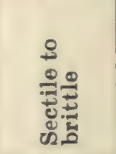 & 营 \\
\hline $\begin{array}{l}\text { o } \\
\text { w } \\
\text { z }\end{array}$ & 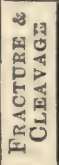 & 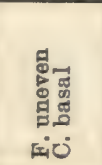 & 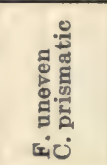 & 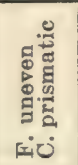 & 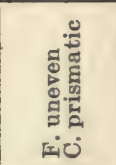 & 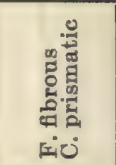 & 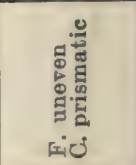 \\
\hline $\begin{array}{l}z \\
0 \\
0 \\
0 \\
w\end{array}$ & 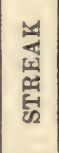 & 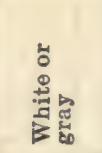 & 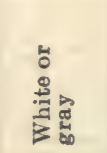 & 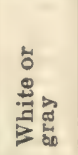 & 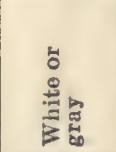 & 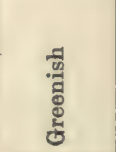 & 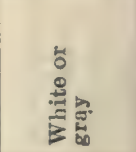 \\
\hline & - & की & 260 & $2 b 0$ & 0 & & bo \\
\hline$\frac{1}{\infty}$ & $\begin{array}{l}\text { 뜡 } \\
\text { 영 } \\
0\end{array}$ & 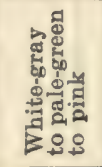 & 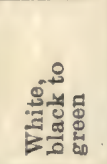 & 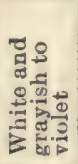 & 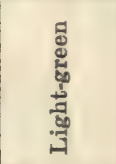 & 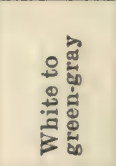 & 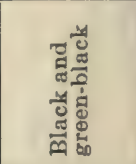 \\
\hline & 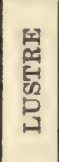 & 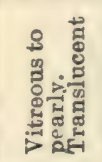 & 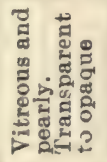 & 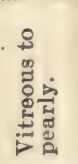 & 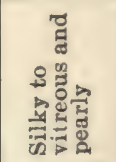 & 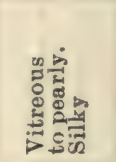 & 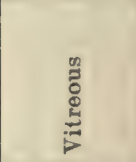 \\
\hline & 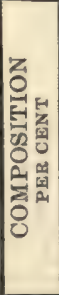 & 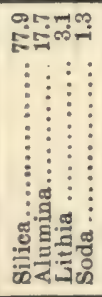 & 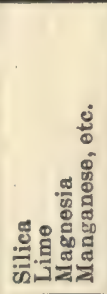 & 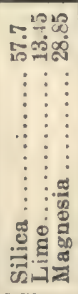 & 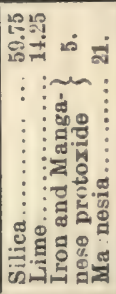 & 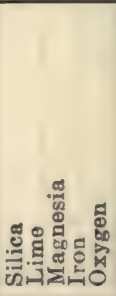 & 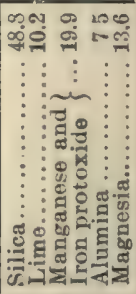 \\
\hline & 蛋 & ڤัँ & 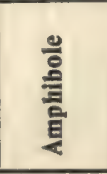 & 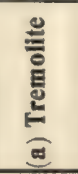 & 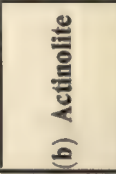 & 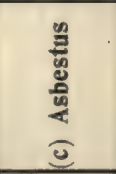 & 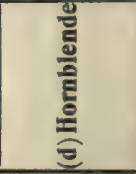 \\
\hline
\end{tabular}


Unsilicates.

133

\begin{tabular}{|c|c|c|c|c|c|c|}
\hline & $\dot{0}^{\circ}$ & 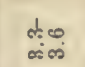 & $\begin{array}{l}+\infty \\
\infty \\
\infty \infty\end{array}$ & స్త & $\begin{array}{l}\text { के } \\
\text { ஸेळ }\end{array}$ & ه్ \\
\hline & 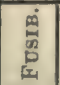 & $\infty$ & मेना & సึ心 & हैं & मरें \\
\hline & 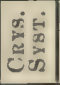 & : & 淐 & $\triangleright$ & 河 & 河 \\
\hline & 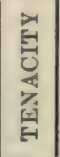 & 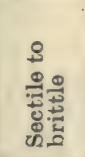 & 䒯 & 竞 & 营 & 焉 \\
\hline & 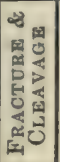 & 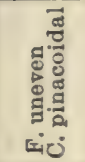 & 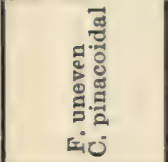 & 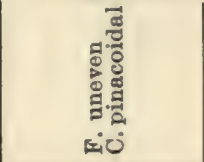 & 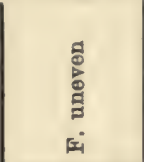 & 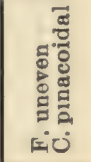 \\
\hline op & 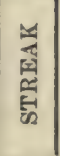 & م & 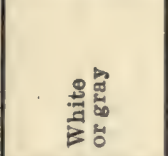 & 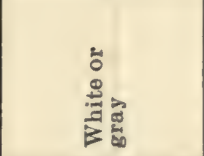 & 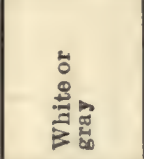 & 范 \\
\hline 10 & $\dot{4}$ & dor & छे & to & $\overrightarrow{2}$ & $a^{\prime 2}$ \\
\hline$\frac{\overline{0}}{2}$ & $\begin{array}{l}\text { 영 } \\
0 \\
0 \\
0 \\
8\end{array}$ & 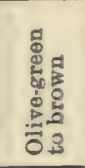 & 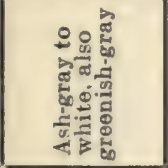 & 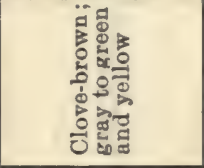 & 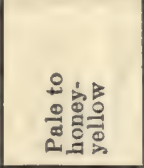 & 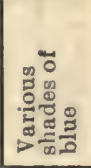 \\
\hline & 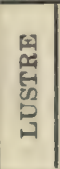 & 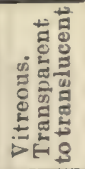 & 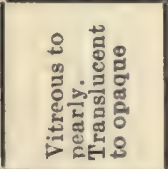 & 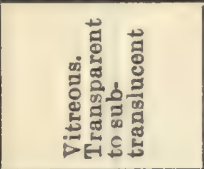 & 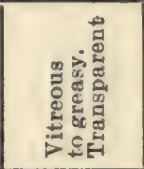 & 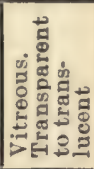 \\
\hline & 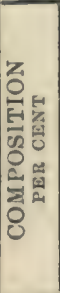 & 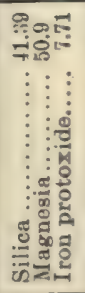 & 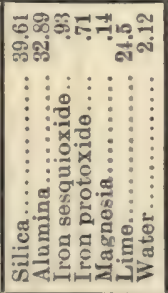 & 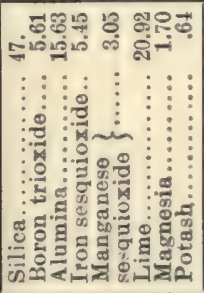 & 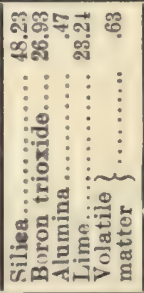 & 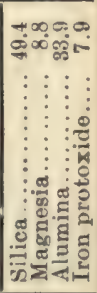 \\
\hline & 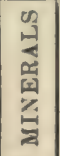 & 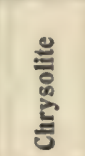 & $\frac{\frac{2}{3}}{\frac{\omega}{2}}$ & 产 & $\begin{array}{l}\text { 茕 } \\
\text { 言 } \\
\text { है }\end{array}$ & 플 \\
\hline
\end{tabular}


Unsilicates.

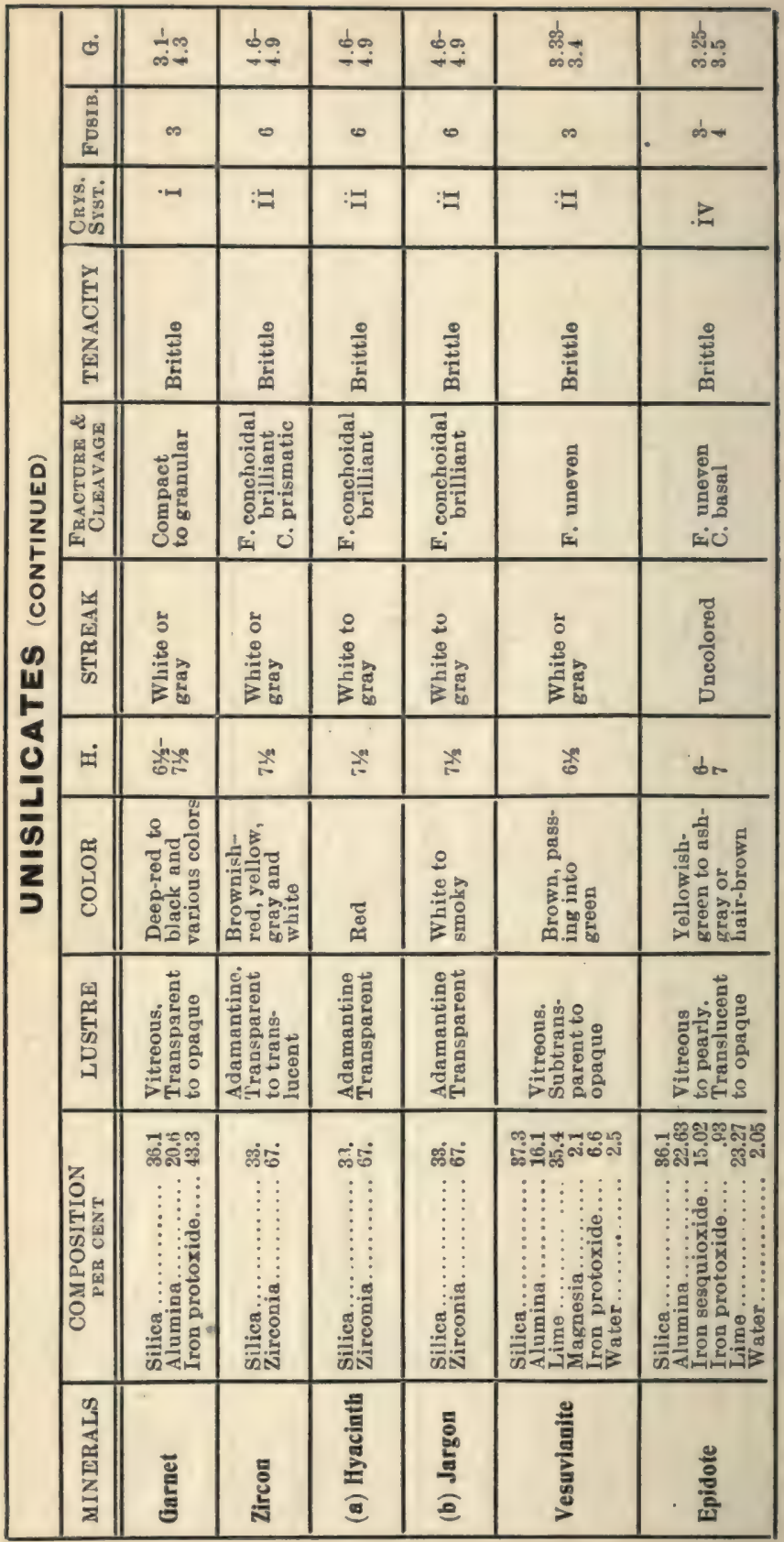


Scapolite Group.

\begin{tabular}{|c|c|c|c|c|c|c|}
\hline & க் & 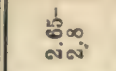 & 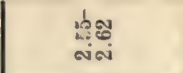 & $\begin{array}{l}\text { से } \\
\text { sici }\end{array}$ & $\begin{array}{l}\text { dran } \\
\text { sioj }\end{array}$ & $\begin{array}{l}\text { bing } \\
\text { vioi }\end{array}$ \\
\hline & 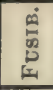 & $\infty$ & + & हैं & ले & - \\
\hline & 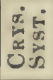 & $: \sqsupseteq$ & $\ddot{=}$ & •rn & $\cdot m$ & 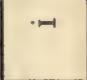 \\
\hline & 至 & 焉 & 䒯 & 营 & 营 & 竞 \\
\hline & 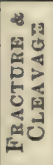 & 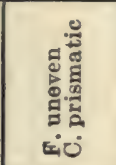 & 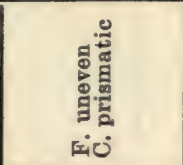 & 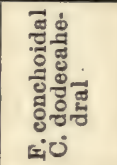 & $\begin{array}{l}\text { 명 } \\
\dot{0} \\
0 \\
0\end{array}$ & 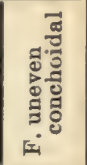 \\
\hline $\begin{array}{l}0 \\
0 \\
0 \\
0\end{array}$ & 究 & 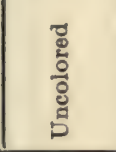 & 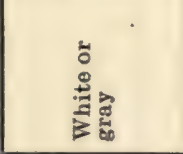 & 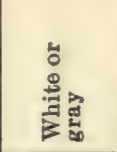 & 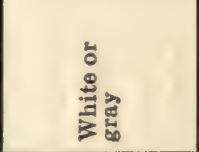 & 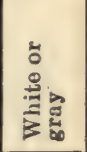 \\
\hline$\underset{t}{\mathbf{w}}$ & $\ddot{I}$ & w bo & $x_{20}^{1}$ & $\infty$ & 10 & $x_{100}^{1}$ \\
\hline $\begin{array}{l}1 \\
0 \\
0 \\
0 \\
0\end{array}$ & $\begin{array}{l}\text { م્ } \\
\text { 경 } \\
\text { ᄋ }\end{array}$ & 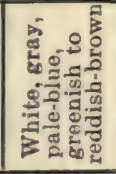 & 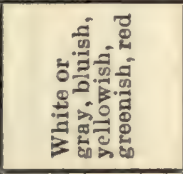 & 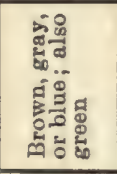 & 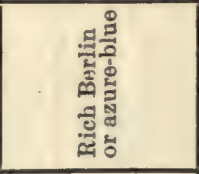 & 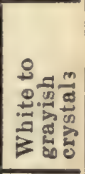 \\
\hline & 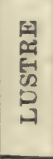 & 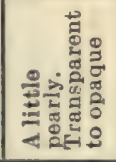 & 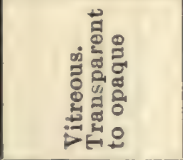 & 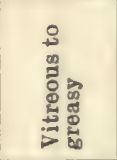 & 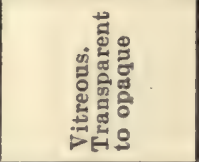 & 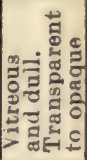 \\
\hline & 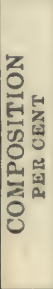 & 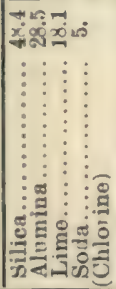 & 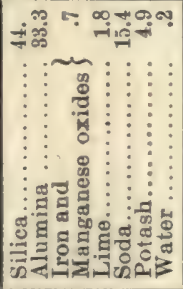 & 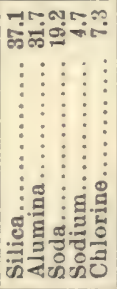 & 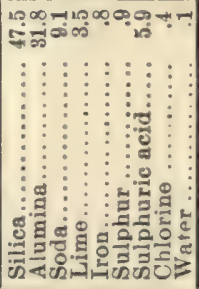 & 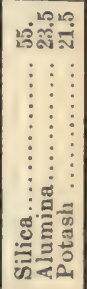 \\
\hline & 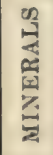 & 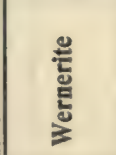 & $\begin{array}{l}\frac{\ddot{E}}{\mathrm{E}} \\
\frac{\mathrm{E}}{\mathrm{E}} \\
z\end{array}$ & 홀 & 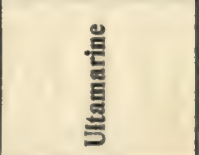 & 플 \\
\hline
\end{tabular}


Feldspar Group.

\begin{tabular}{|c|c|c|c|c|c|c|c|c|}
\hline & $\dot{0}$ & 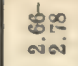 & $\begin{array}{l}\frac{1}{6} \\
\text { oiaj }\end{array}$ & $\stackrel{8}{8}$ & 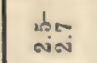 & $\begin{array}{l}\text { मृष्ठ } \\
\text { aivi }\end{array}$ & 战 & 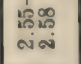 \\
\hline & 畧 & $\underset{*}{*}$ & $1 *$ & $1 \times$ & $1 *$ & 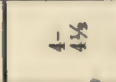 & 10 & 10 \\
\hline & 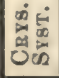 & $>$ & $>$ & $>$ & $>$ & $>$ & $>$ & . \\
\hline & है & 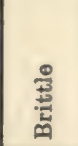 & 营 & 莺 & 营 & 营 & 莣 & 莺 \\
\hline & 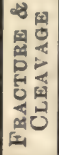 & 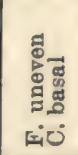 & 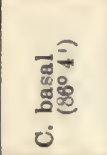 & 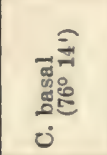 & 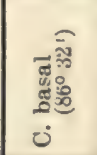 & 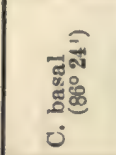 & 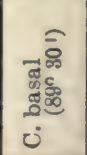 & 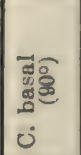 \\
\hline$\frac{0}{2}$ & 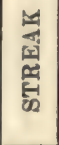 & 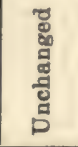 & 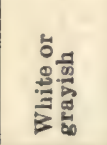 & 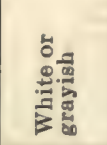 & 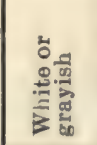 & 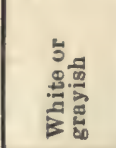 & 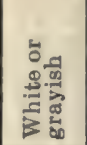 & 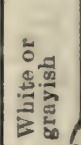 \\
\hline$a$ & $\ddot{H}$ & ando & खें & $\infty$ & $\infty$ & de & 0 & $\infty$ \\
\hline $\begin{array}{l}0 \\
0 \\
0 \\
11 \\
11\end{array}$ & $\begin{array}{l}\text { 융 } \\
\text { 옹 } \\
8\end{array}$ & 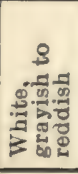 & 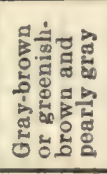 & 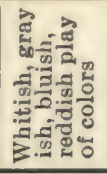 & 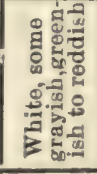 & 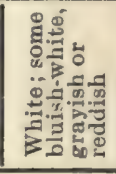 & 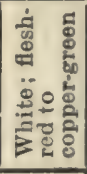 & 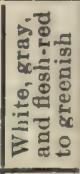 \\
\hline & 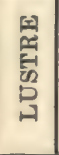 & 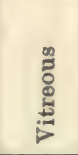 & 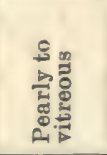 & 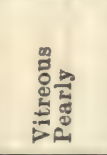 & 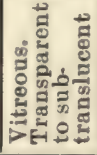 & 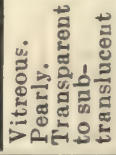 & 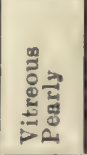 & 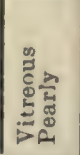 \\
\hline & 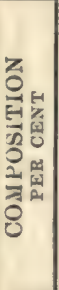 & 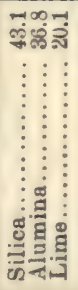 & 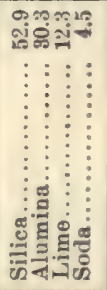 & 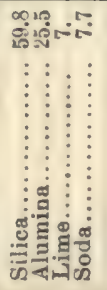 & 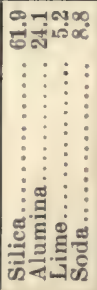 & 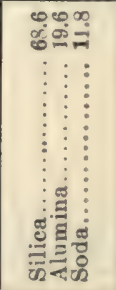 & 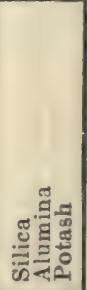 & 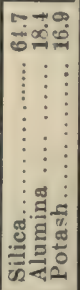 \\
\hline & 晃 & $\begin{array}{l}\text { 를 } \\
\text { 를 } \\
\text { 을 }\end{array}$ & 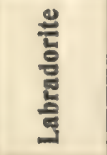 & 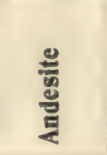 & 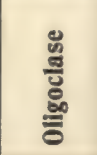 & 产 & 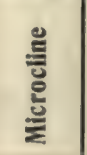 & $\begin{array}{l}\text { 맴 } \\
\text { 을 } \\
\text { 를 }\end{array}$ \\
\hline
\end{tabular}


Mica Group.

\begin{tabular}{|c|c|c|c|c|c|c|}
\hline & வீ & 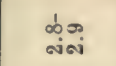 & فำ & i. & को. & $\stackrel{\omega}{\dot{\omega}}$ \\
\hline & 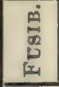 & os & 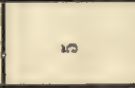 & -1 & సิ่ & सi⿺ \\
\hline & 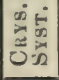 & \pm & . & . & 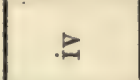 & . \\
\hline & 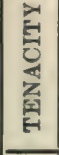 & 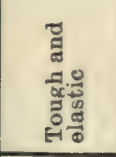 & 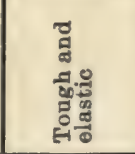 & 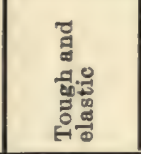 & 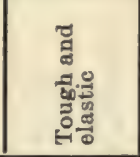 & 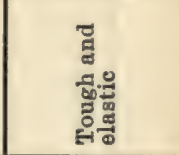 \\
\hline & 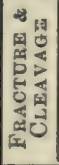 & 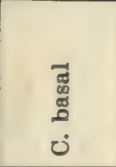 & 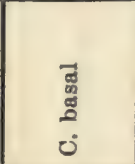 & 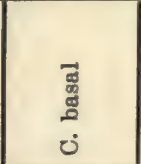 & 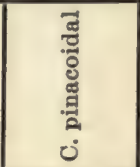 & 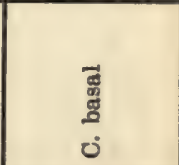 \\
\hline$\frac{2}{0}$ & 省 & 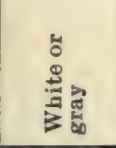 & 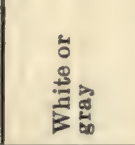 & 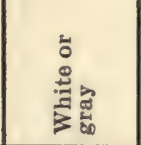 & 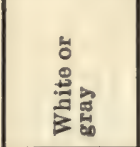 & 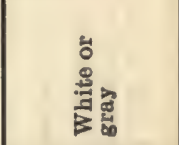 \\
\hline$\frac{a}{0}$ & 봄 & ix & ते & $\overbrace{\infty}^{1}$ & $\infty$ & 党办 \\
\hline$\frac{8}{5}$ & $\begin{array}{l}\text { 뗘 } \\
0 \\
-1 \\
0 \\
0\end{array}$ & 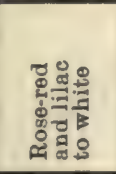 & 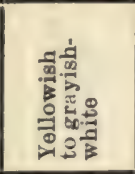 & ङี & 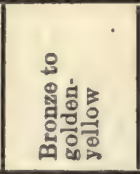 & 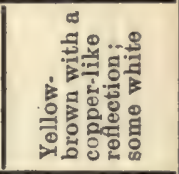 \\
\hline & 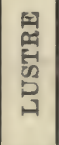 & 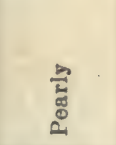 & 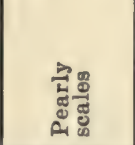 & 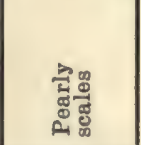 & & 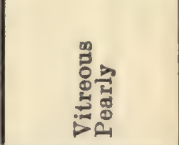 \\
\hline & 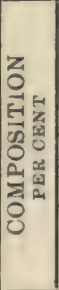 & 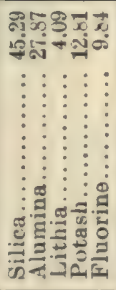 & 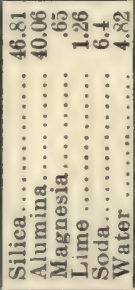 & 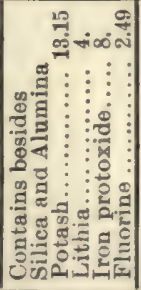 & 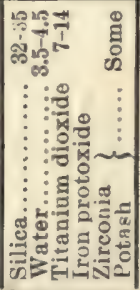 & 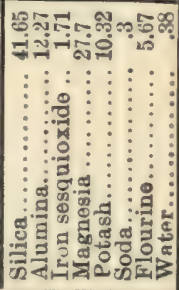 \\
\hline & 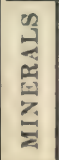 & $\begin{array}{l}\text { 쁳 } \\
\frac{\text { 믈 }}{3}\end{array}$ & 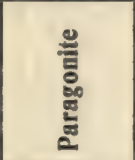 & $\begin{array}{l}\text { 츨 } \\
\frac{2}{0} \\
\frac{2}{2}\end{array}$ & $\begin{array}{l}\text { 롳 } \\
\text { 츨 } \\
\text { 흘 }\end{array}$ & 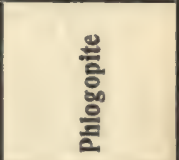 \\
\hline
\end{tabular}


Subsilicates.

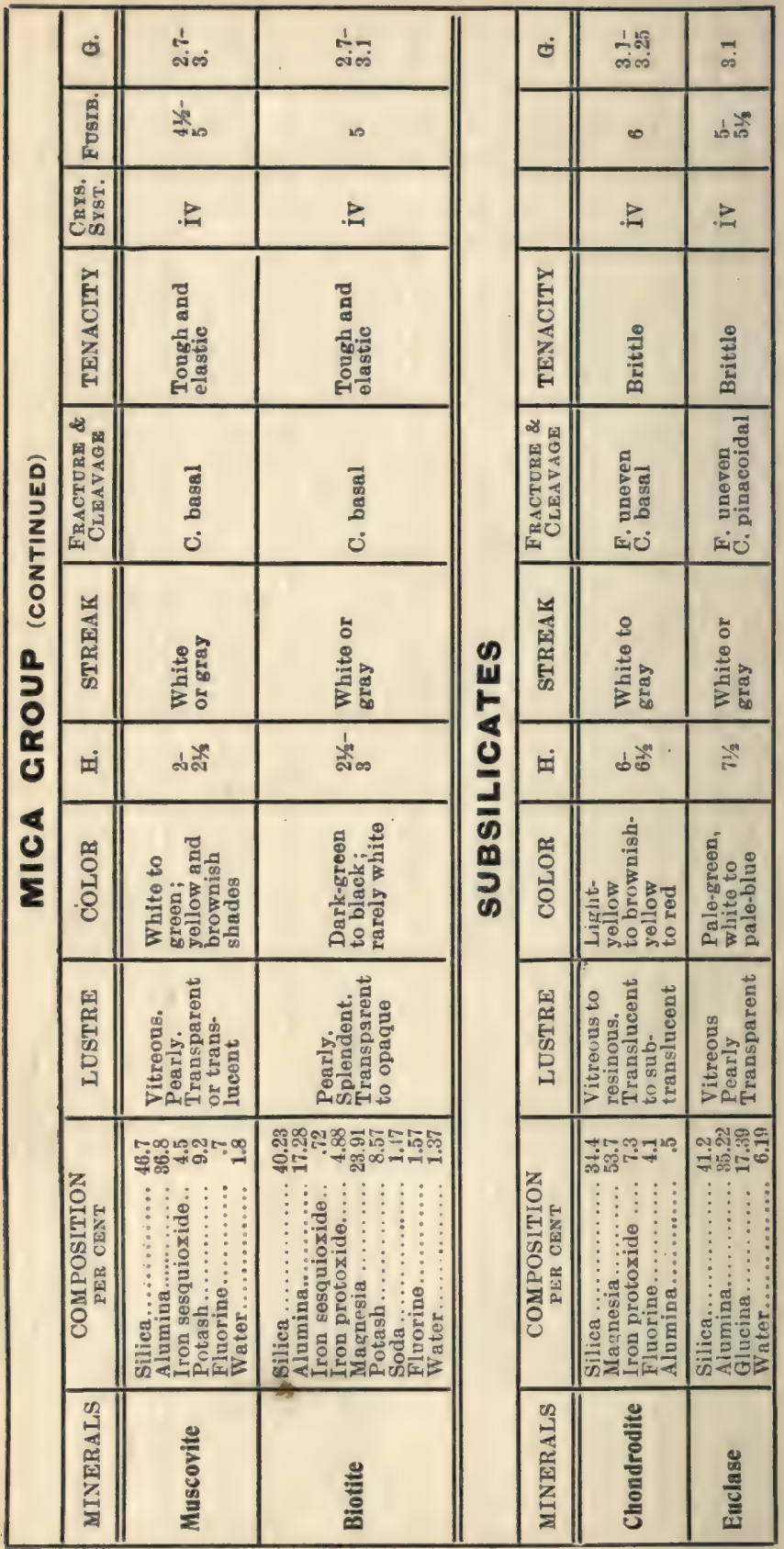


Subsilicates.

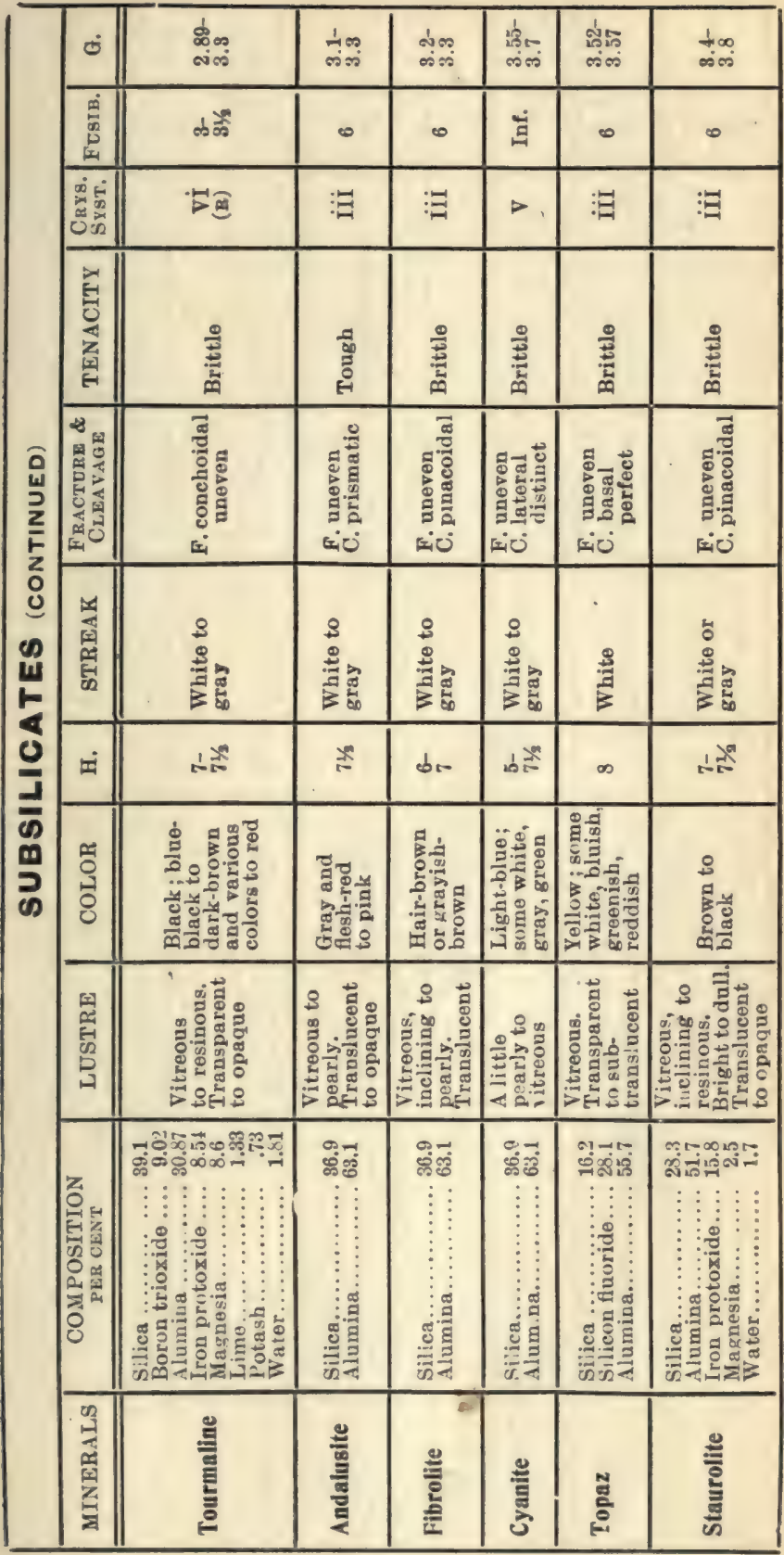


Hydrous Subsilicates.

\begin{tabular}{|c|c|c|c|c|c|c|c|}
\hline \multirow{4}{*}{ - } & ه & $\stackrel{d}{\text { ive }}$ & $\frac{+10}{\cos ^{\circ}}$ & & க் & 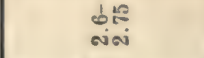 & $\begin{array}{l}\text { br? } \\
\text { งici }\end{array}$ \\
\hline & 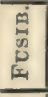 & ผลે & + & & 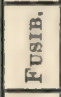 & in $x^{*}$ & 1ค \\
\hline & 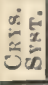 & . & : & & 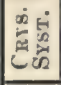 & 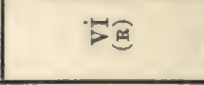 & $\stackrel{R}{*}$ \\
\hline & 空 & 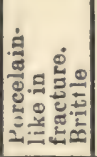 & 竎 & $\frac{1}{0}$ & 酎 & 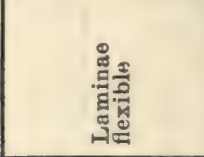 & 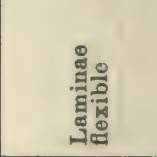 \\
\hline \multirow{7}{*}{ 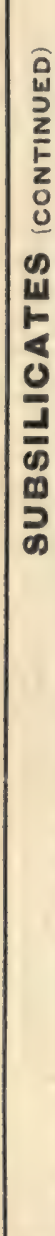 } & 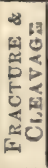 & 这 & 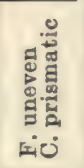 & $\begin{array}{l}\frac{\alpha}{0} \\
w \\
\underline{v}\end{array}$ & 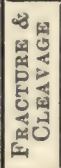 & 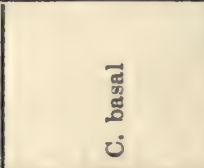 & 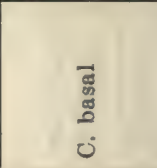 \\
\hline & 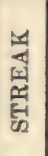 & 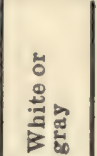 & $\begin{array}{l}\overline{0} \\
\vdots \\
\frac{0}{0} \\
0 \\
0\end{array}$ & $\begin{array}{l}0 \\
\frac{1}{0}\end{array}$ & 䇋 & 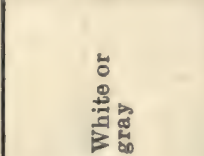 & 宫 \\
\hline & 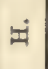 & का & เด & uㅏㅁ & $\dot{4}$ & ลื & ปล \\
\hline & $\begin{array}{l}\text { oี } \\
0 \\
0 \\
0\end{array}$ & 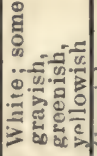 & 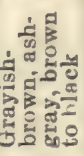 & $\begin{array}{l}0 \\
\overline{0} \\
0\end{array}$ & 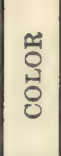 & 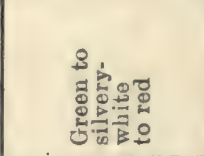 & 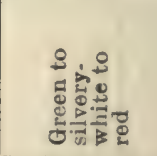 \\
\hline & 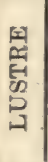 & 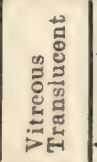 & 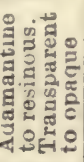 & $\begin{array}{l}0 \\
0 \\
0\end{array}$ & 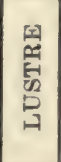 & 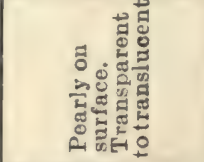 & 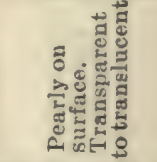 \\
\hline & 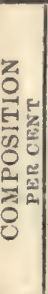 & 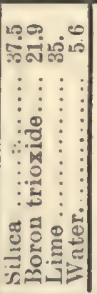 & 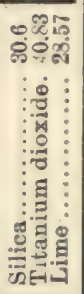 & $\frac{a}{I}$ & 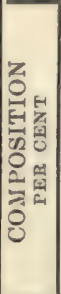 & 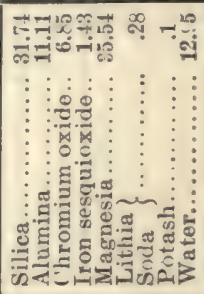 & 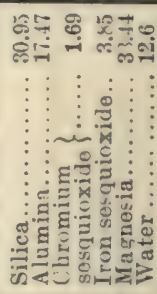 \\
\hline & 语 & है & 产 & + & 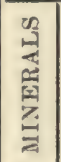 & 总 & $\frac{0}{\frac{\pi}{3}}$ \\
\hline
\end{tabular}


Hydrous Subsilicates.

\begin{tabular}{|c|c|c|c|c|c|c|c|}
\hline \multirow{6}{*}{ 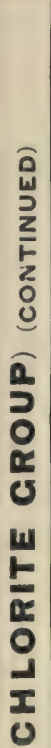 } & ه & $\begin{array}{l}\text { ठ゙ } \\
\text { eळ }\end{array}$ & $\overrightarrow{\text { N }}$ & $\begin{array}{l}\text { సูm } \\
\text { siov }\end{array}$ & $\infty$ & 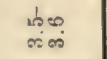 & งก่ \\
\hline & $\begin{array}{l}\text { 愚 } \\
\text { 需 }\end{array}$ & מ่⿻ & $*$ & $+\frac{0}{*}$ & $\frac{1}{2}=$ & $\infty$ & $\infty$ \\
\hline & 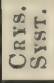 & 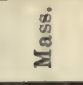 & 浔 & 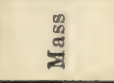 & 洁 & . & $\ddot{\nu}$ \\
\hline & 离 & 营 & 商 & 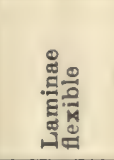 & 竞 & 营 & 竎 \\
\hline & 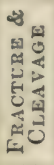 & 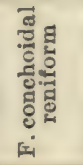 & 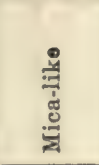 & 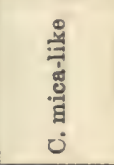 & 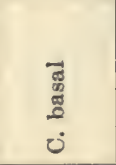 & 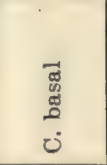 & 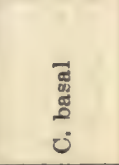 \\
\hline & 选 & 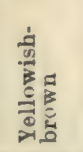 & & 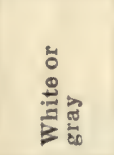 & 告 & & \\
\hline U & \pm & $\infty$ & $\infty$ & $\Delta \approx$ & $x^{\prime}=$ & $x_{-\infty}^{1}$ & I-N \\
\hline$\frac{1}{10}$ & $\begin{array}{l}\text { 져 } \\
\text { م્ } \\
0 \\
0\end{array}$ & 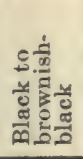 & 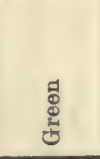 & 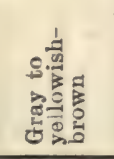 & 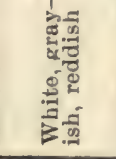 & 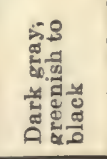 & 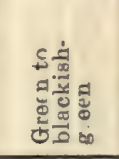 \\
\hline $\begin{array}{l}5 \\
0 \\
0 \\
0\end{array}$ & 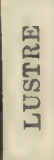 & 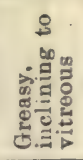 & 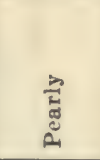 & 岕 & 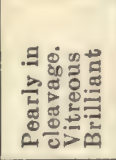 & 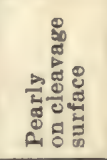 & 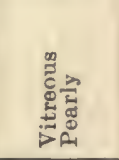 \\
\hline $\begin{array}{l}2 \\
2 \\
2\end{array}$ & 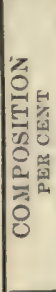 & 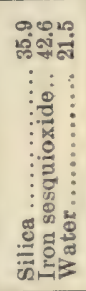 & 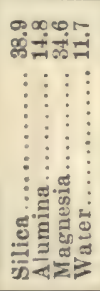 & 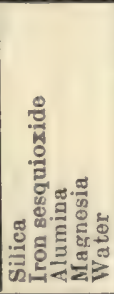 & 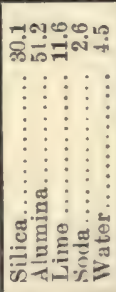 & 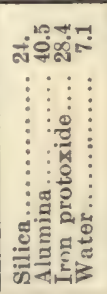 & 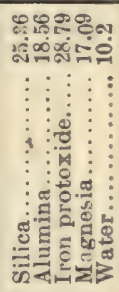 \\
\hline & 告 & $\begin{array}{l}\text { 흘 } \\
\text { 핼 } \\
\text { 믈 }\end{array}$ & 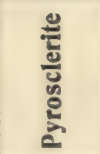 & 菲 & 总 & $\begin{array}{l}\text { 믈 } \\
\text { 흔 } \\
\text { 흠 }\end{array}$ & 흘 \\
\hline
\end{tabular}


Hydrous Silicates.

\begin{tabular}{|c|c|c|c|c|c|c|c|c|}
\hline & $\dot{0}$ & فี & 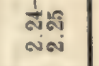 & $\begin{array}{l}\text { ลี } \\
\text { ง }\end{array}$ & $\begin{array}{l}\text { of } \\
\text { जiai }\end{array}$ & i & $\frac{1}{\text { ลini }}$ & $\stackrel{\text { Na }}{\text { N }}$ \\
\hline & $\begin{array}{l}\text { 象 } \\
\text { 总 } \\
\text { 点 }\end{array}$ & ฟ่ง & ลี้ & ळ & $\infty$ & $\infty$ & $\infty$ & - \\
\hline & 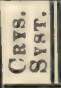 & :決 & : & . In & 㐫 & : & 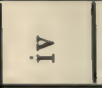 & $D$ \\
\hline & 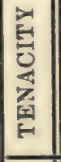 & 竎 & 車 & 遂 & 莣 & 总 & 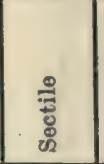 & 总 \\
\hline $\begin{array}{l}\frac{2}{0} \\
0 \\
0\end{array}$ & 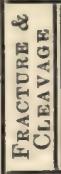 & 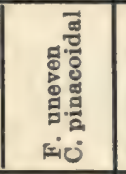 & 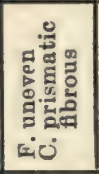 & 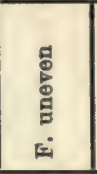 & 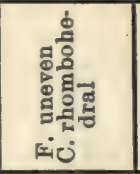 & 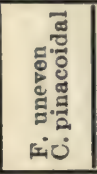 & 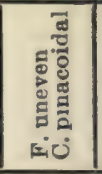 & 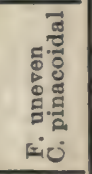 \\
\hline 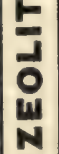 & 瓷 & 晃 & 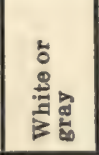 & 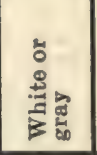 & $\begin{array}{l}9 \\
0\end{array}$ & 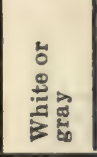 & 总 & 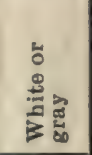 \\
\hline$\infty$ & 田 & बक्षे & 的族 & 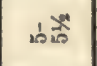 & +100 & 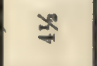 & ๓े & $\stackrel{1}{\infty}$ \\
\hline$\frac{1}{1}$ & 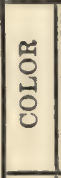 & $\begin{array}{l}0 \\
0 \\
\end{array}$ & 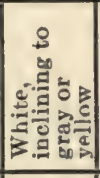 & 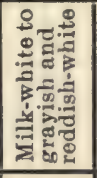 & 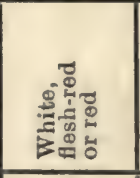 & 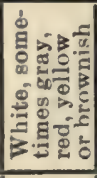 & 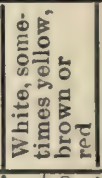 & 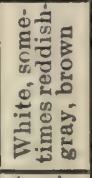 \\
\hline $\begin{array}{l}0 \\
0 \\
0 \\
0\end{array}$ & 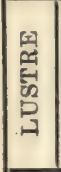 & 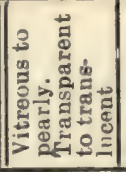 & 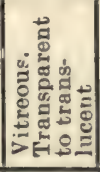 & 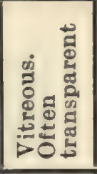 & 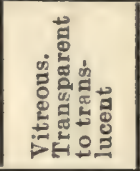 & 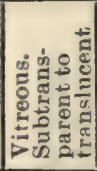 & 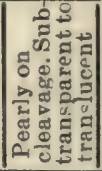 & 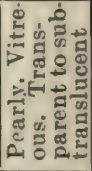 \\
\hline$I$ & 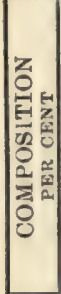 & 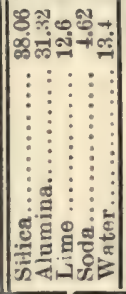 & 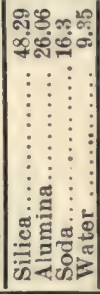 & 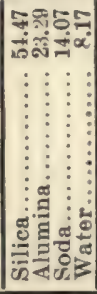 & 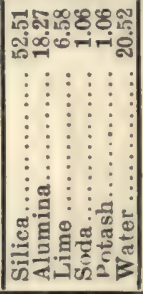 & 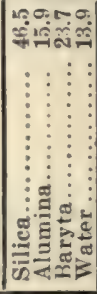 & 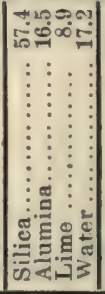 & 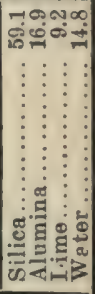 \\
\hline & 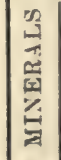 & $\begin{array}{l}\text { 플 } \\
\text { 品 } \\
\text { 总 }\end{array}$ & $\frac{\text { 를 }}{\text { 를 }}$ & $\frac{\text { 블 }}{\frac{y}{2}}$ & $\begin{array}{l}\text { 롤 } \\
\text { 률 } \\
\text { 폻 }\end{array}$ & 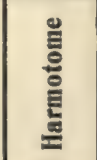 & 总 & 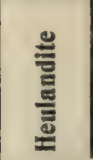 \\
\hline
\end{tabular}


Hydrous Silicates.

\begin{tabular}{|c|c|c|c|c|c|c|}
\hline & க் & 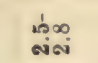 & 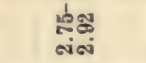 & $=$ & ๙่. & $\begin{array}{l}\text { do } \\
\text { Nidi }\end{array}$ \\
\hline & 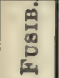 & 10 & $\infty$ & D & N่es & b䃌 \\
\hline & & : & 浔 & 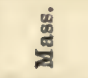 & 总 & 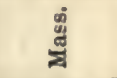 \\
\hline & 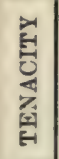 & 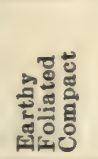 & 营 & 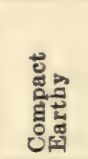 & 蔗 & 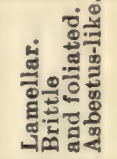 \\
\hline & 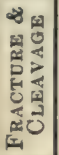 & 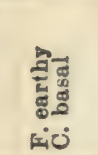 & 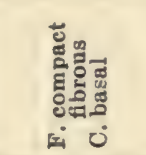 & $\begin{array}{l}\text { 동 } \\
0 \\
0 \\
0\end{array}$ & ⿸\zh14㔾 & 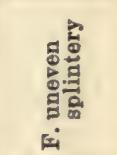 \\
\hline $\begin{array}{l}\infty \\
w \\
1 \\
4 \\
0\end{array}$ & 承 & 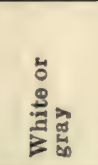 & 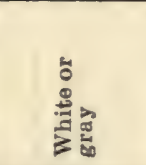 & $\frac{3}{3}$ & & 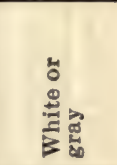 \\
\hline $\bar{\sigma}$ & 壮 & $\dot{1}=$ & -100 & ล่ & o & ๙े \\
\hline $\begin{array}{l}0 \\
0 \\
0 \\
0 \\
0\end{array}$ & $\begin{array}{l}\text { 용 } \\
\text { 今ం } \\
0\end{array}$ & 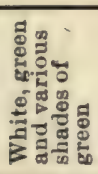 & 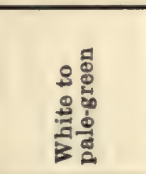 & 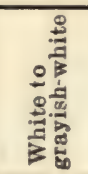 & 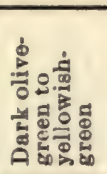 & 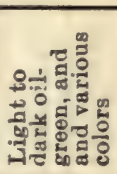 \\
\hline & 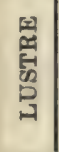 & 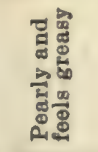 & 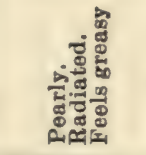 & 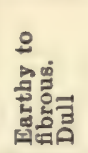 & ีㅗㅗ & 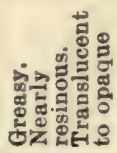 \\
\hline & 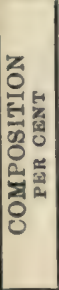 & 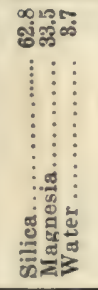 & 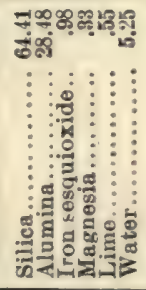 & 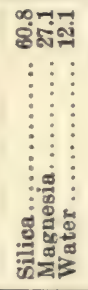 & 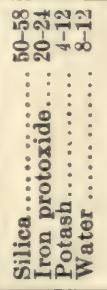 & 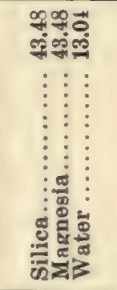 \\
\hline & 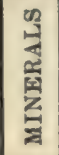 & $\stackrel{\text { 를 }}{\leftarrow}$ & $\frac{y}{\frac{3}{2}}$ & 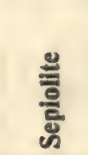 & $\begin{array}{l}\text { 를 } \\
\text { 를 } \\
\text { 응 }\end{array}$ & 를 \\
\hline
\end{tabular}


Hydrous Silicates.

\begin{tabular}{|c|c|c|c|c|c|c|}
\hline & $0^{\circ}$ & 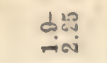 & & 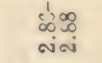 & 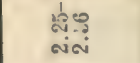 & فे \\
\hline & 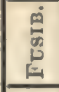 & two & & $\frac{1}{\omega} \infty$ & ลें & $\infty$ \\
\hline & 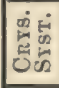 & 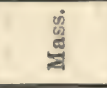 & 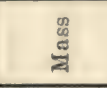 & 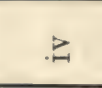 & : & $:[\bar{I}$ \\
\hline & 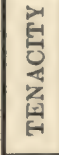 & 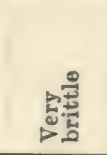 & & 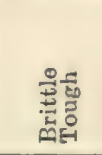 & 营 & 芦 \\
\hline $\begin{array}{l}\text { 是 } \\
2 \\
z \\
\text { z } \\
z \\
0 \\
0\end{array}$ & 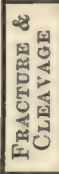 & 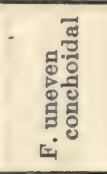 & & 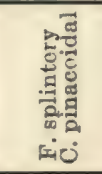 & 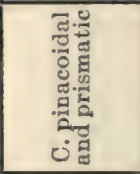 & 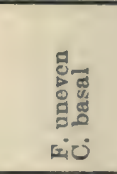 \\
\hline $\begin{array}{l}0 \\
\text { un } \\
15 \\
0\end{array}$ & 点 & 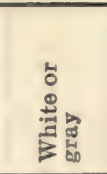 & 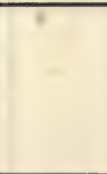 & 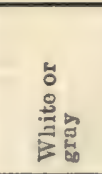 & 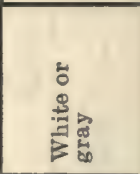 & 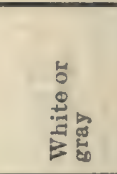 \\
\hline$\frac{d}{0}$ & $\ddot{4}$ & ब่ & & 20 & $\frac{1}{m+1}$ & $\neq 10$ \\
\hline $\begin{array}{l}0 \\
0 \\
0 \\
0\end{array}$ & 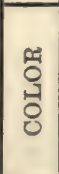 & 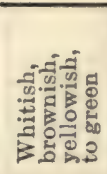 & 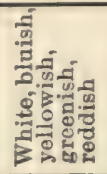 & 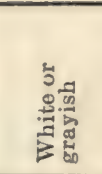 & 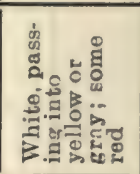 & 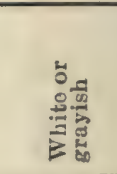 \\
\hline$x$ & 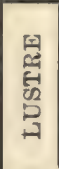 & 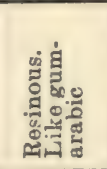 & 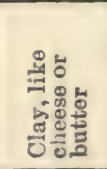 & 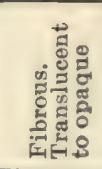 & 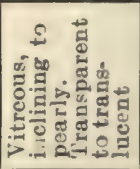 & 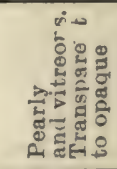 \\
\hline & 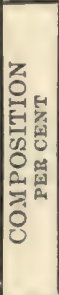 & 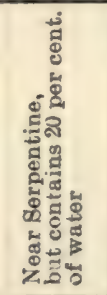 & 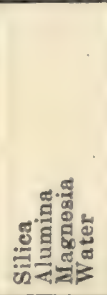 & 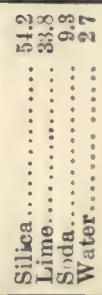 & 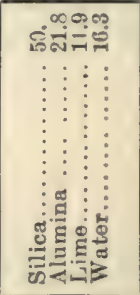 & 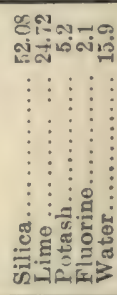 \\
\hline & 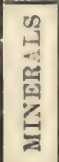 & 产 & $\begin{array}{l}\stackrel{y}{\bar{~}} \\
\text { 핶 }\end{array}$ & 总 & 를 & $\frac{0}{\frac{3}{2}}$ \\
\hline
\end{tabular}


Hydrous Silicates.

\begin{tabular}{|c|c|c|c|c|c|c|}
\hline & ن் & 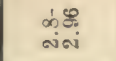 & $\begin{array}{l}1 \\
0 \\
-12 \\
-1\end{array}$ & $\begin{array}{l}\frac{1}{4} 0 \\
\text { ani }\end{array}$ & $\underset{d r}{d r}$ & $\vec{a}$ \\
\hline & 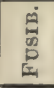 & $\ddot{a t}$ & 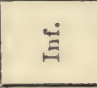 & 0 & & . \\
\hline & 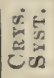 & 渄 & 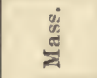 & $:$ & 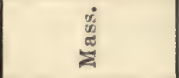 & $\varpi$ \\
\hline & 恶 & 莣 & 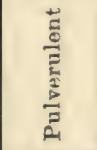 & 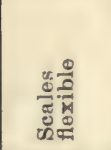 & & 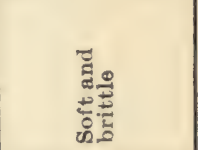 \\
\hline $\begin{array}{l}\frac{1}{2} \\
z \\
z \\
z \\
0\end{array}$ & 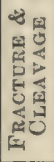 & 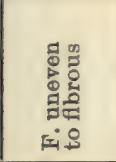 & . & 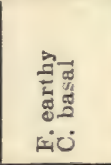 & & \\
\hline $\begin{array}{l}0 \\
1 \\
1 \\
0 \\
0\end{array}$ & 군 & 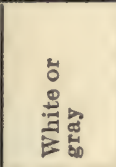 & & है & & . \\
\hline$\overline{0}$ & I & के के & $\infty$ & मे & तें & - \\
\hline $\begin{array}{l}0 \\
0 \\
0 \\
0 \\
0\end{array}$ & 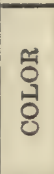 & 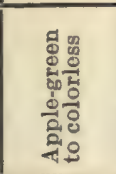 & 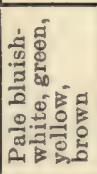 & 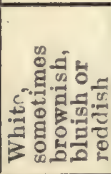 & 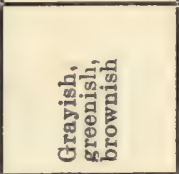 & 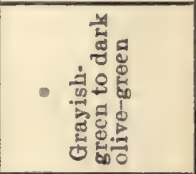 \\
\hline & 国 & 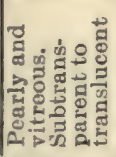 & 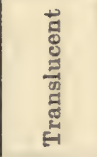 & 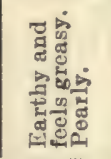 & 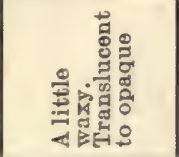 & 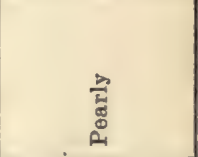 \\
\hline & 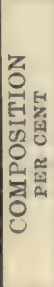 & 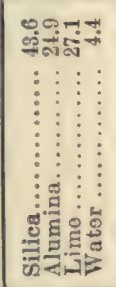 & 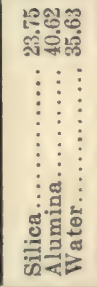 & 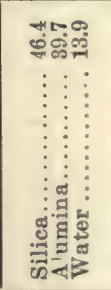 & 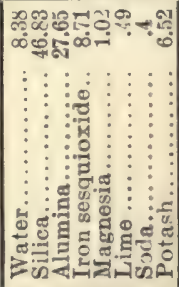 & 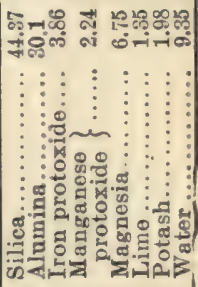 \\
\hline & 售 & 章 & $\begin{array}{l}\text { 듶 } \\
\text { 흘 } \\
\text { 을 }\end{array}$ & 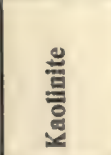 & 플 & 氖 \\
\hline
\end{tabular}

Dana's "System of Mineralogy."

Brush \& Penfield's "Determinative Mineralogy and Blowpipe Analysis." 



\section{PART IV. \\ DNaming Rocks}

Glossary of Mining Terms 



\section{PART IV.}

\section{NAMING ROCKS.}

EXPLANATION OF TERMS

Rocks are mineral aggregates, or in other words they are inorganic substances formed by the chemical or mechanical union of two or more minerals. Rocks, like minerals, exhibit different forms and varlous physical and chemical characteristics, certain terms are applied to rocks in accordance with these features, by which their appearance and structure are broadly expressed. For example: A rock, when sandy, is said to be arena. ceous; or when it is clayey it is called argillaceous; when it contains much lime it is called calcareous; or much silica, it is termed silicious.

A sedimentary rock is one which has resulted from the chemical or mechanical precipitation of sediment.

An aqueous rock has been formed by the action of water.

An igneous rock is one which has its origin in fire, either directly or indirectly.

A stratified rock is one in which the lines of deposit are clearly marked in layers and such layers point to aqueous origin.

An unstratified rock, on the contrary, has no such lines, and with one or two exceptions such rocks are of igneous origin.

Metamorphic rocks are those in which some change has been induced due to crystallization after deposit. The term metamorphic is generally restricted to rock formerly of sedimentary or aqueous origin. 
Crystalline is a term employed to express the structure of rocks. Many rocks which appear to the unaided eye to be compact or massive, under the microscope often exhibit complete crystalline structure.

Glassy, or vitreous, are terms used to express the presence of glass which occurs in the bases of most all igneous rocks.

Porphyritic is the term employed to denote a structure in which a few crystals have separated themselves out and crystallized apart from the ground mass of the rock; a rock is said to be porphyritic especially when these crystals consist of feldspar.

Amygdaloidal rocks are those in which the almondshaped vesicles have been filled up by other minerals brought in, probably by percolating water, such minerals are termed "zeolites."

Vesicular rocks are those containing spherical cavaties.

Fluidal is a term applied to rocks of a wavy or streaky appearance; it is common in rhyolyte, and marks the movement of particles in a once molten rock.

Schistose is the name given to a structure which has been induced in rocks by metamorphism. The structure consists in the arrangement in wavy, irregular lines and layers of the different minerals.

Oolitic-Pisolitic-Oolitic is the term used to explain the structure of a rock which is like the compact roe of a fish. When the grains are large the rock is called "pisolitic."

UNSTRATIFIED, METAMORPHIC AND ERUPTIVE ROCKS.

Granite-Composition, quartz, orthoclase and mica, metamorphic and eruptive. The chemical composition of granite agrees very nearly with that of some sand- 
stones and clays, and there seems to be no reason to doubt that granites are merely altered portions of the strata-portions which have been subjected to intense heat, movement and pressure. Granites occur either as intrusive veins or in hills and masses, varying from a small boss up to a large mountain range extending for many miles. It has been forced up through the earth's solid crust in a pasty, moist condition, from which, under varying circumstances, it has consolidated and crystallized. Granite is always newer or younger than the rocks it fissures or overlies; it is easily recognized and widely known; they are all visibly crystalline, the feldspar crystals varying from minute flakes up to crystals many inches in length. Granite passes into gneiss by pressure; the gneiss is virtually a granite of a schistose structure, the component minerals having arranged themselves more or less in layers.

$$
\text { G. }=2.5-2.8 \text {. Silica } 70 \text {-72 p. c. }
$$

Syenyte Granite-A hard compact granite of a dark greenish to grayish color, the color resulting from the presence of the mineral hornblende, which partly replaces the micas.

Protogine is a softish granite containing pale green stains of chlorite and blotches of talc. It is gneiss-like in structure.

Luxulianyte is a softish flesh-colored granite in which the mica is partly replaced by the impure subsilicate of alumina and tourmaline.

Granityte is a hard granite containing biotite mica in considerable quantities.

Granulyte is a granite, often soft and easily decomposed, in which the quartz is very scarce or entirely 
absent. G. $=2.6-2 . \%$. Silica $\approx 0-80$ p. c. It contains no mica.

Greisen is a foliated soft granite with little or no feldspar. It is a schistose micaceous quartz rock.

Granite-porphyry is a granite in which, through some influence not understood, large, isolated crystals separate themselves from the mass of felsite, which remains in a pasty magma, Composition, felsite, quartz, feldspar, mica or chlorite.

Quartz-porphyry-Composition same as above, with no mica or chlorite, it is a hard, compact rock of various colors, generally gray to brown.

Felsyte-A many-colored rock, from gray to bluish to brown and red; compact and very hard. It is a rapidly cooled granite paste, containing crystals of quartz and feldspar.

Pitchstone-A dark, glassy rock, with numbers of small crystals of glassy feldspar, and sometimes crystals of sanidin, quartz and mica. It is a glassy feldsite which has cooled rapidly.

Rhyolyte, or quartz trachyte-A compact feldsite with glass in the base, and quartz crystals and occasionally mica. Rhyolytes are of many colors and textures, breaking with a rough fracture. They frequently exhibit wavy lines of structure (fluidal), caused by the arrangement in lines or layers of mineral aggregates or colored obsidian. They contain a large amount of quartz, which has resulted from the excess of silica not required to complete the feldspar crystals.

Pearlyte is a rapidly cooled rhyolyte. 
Obsidian-A black, brown, red or green volcanic glass, generally or most always of some dark color, looking and breaking like dark bottle glass.

Pumice-stone-A light, spongy, grayish rock, which floats on water. It is a volcanic foam, so to speak.

Syenyte-A metamorphic and eruptive rock closely related to granite. It consists of orthoclase, hornblende, oligoclase, mica, nepheline, augite, and sometimes zircon. It is generally a grayish and flesh-colored rock, from the colors induced in it by its chief typical minerals, orthoclase and hornblende. Zircon syenyte is rare and occurs chiefly in intrusive dykes.

\section{$\mathrm{G} .=2.7-2.9$. Silica $58-63$ p. c.}

Porphyryte-A volcanic rock, close-grained and breaking with an even fracture. It is formed almost entirely of feldspar with some magnetite. It is known by various names, according to the development of certain minerals. Mica-porphyryte has much mica; hornblende-porphyryte, much hornblende, etc. Porphyryte was largely outpoured during the carboniferous age, and would appear to be an imperfectly crystallized lava.

Trachyte-A rock of various colors, hard, but brittle, with rough fracture. It is the volcanic representative of syenyte. It was originally poured out as a thick, viscous lava-stream. Sanidin is always well represented, which forms glassy crystals in the base. The minerals hornblende, augite, apatite, magnetite and titanite are often present. It is an orthoclase rock, with also oligoclase and crysolite. G. $=2.6-2 . \%$. Silica $60-64$ p. c.

Minette-A volcanic rock consisting of biotite, or- 
thoclase, nepheline and sodalite. It is a dark-colored felsitic rock in which biotite is an abundant mineral. It occurs chiefly in dykes and intrusive veins.

\section{Silica $50-65$ p. c.}

Phonolyte-Clinkstone-A hard, compact rock, which rings under the hammer. Gray, grayish-blue, to brownish. It turns white by weathering. It consists of the minerals orthoclase (sanidin), nepheline, hornblende and titanite. It is a nepheline-trachyte, and often contains large and well-defined crystals of amphibole. Zeolites often occur, filling cavities in the mass. Both sanidin and nepheline show very clear, and their presence admit of approximate indentification of the rock. The Cripple Creek District, Colo., is a noted locality of this rock, where, in structure, it is more or less slaty.

Quartz-dioryte-Both a metamorphic and eruptive rock, consisting of quartz and plagioclase with hornblende. It is a very tough grayish to greenish-white rock, rich in silica. Its texture varies from coarse to fine-grained, often porphyritic. Called also greenstone. $\mathrm{G} .=2.66$-3. Silica $50-64$ p. c.

Quartz-porphyry-An emuptive grayish to greenish rock, consisting of minutely crystalline paste of quartz, oligoclase, hornblende, with large crystals of the same, and titanite. It occurs in large masses, having probably been ejected through fissures. It is tough and coarse in fracture.

Dacyte (quartz-andesyte)-An eruptive, dull, grayish-green rock, compact but not hard, and consists of feldspar, hornblende, quartz, small crystals of oligoclase, sanidin and magnetite.

Silica $65-70$ p. c. Often graduates into orthoclase rocks. 
Dioryte-An eruptive, coarse to fine-grained, compact rock; like syenyte in structure; color, light-gray and green to dark greenish black. Its typical constituents are: Orthoclase, feldspar, oligoclase and hornblende, with sometimes labradorite, apatite, magnetite and pyrite. When containing quartz, it is known as quartz-dioryte. The hornblende is easily recognized in the form of small needles, and the feldspar is more often flesh-colored than white. It occurs chiefly in wide dykes and fissures. G. $=2.66-3$. Silica $50-64$ p. c.

Andesyte-An eruptive, bard, compact rock, consisting of the minerals, andesı te, crystals of augite or hornblende, biotite and magnetite, with labradorite as the chief feldspar. Augite-andesyte is a dark-gray nearly black rock, with dark-colored crystals of augite.

Hornblende-andesyte is a pale-gray, compact rock, where the dark-green hornblende crystals occur in small columnar forms. Occasionally the hornblende appears surrounded by pale-green stains, indicating its alteration into chlorite. Andesytes are of wide occurence. They have been poured out from dykes and fissures. G. $=2.6-$ 2.7. Silica 59-63 p. c. Has more or less the aspects of trachyte.

Gabbro-An eruptive rock, occurring in intrusive dykes and sheets. Its color varies from dark-gray, blackish to brown, rusty red, and sometimes bright spangles of mica. 1ts chief mineral constituents are: Labradorite, diallage or pyroxene. Magnetite and mica are accessory minerals. The kind known as olivinegabbro contains the mineral olivine as a constituent. Hypersthenite is a closely-related rock. Gabbro is widely distributed and often is associated with serpentine. Granite-like in texture. G. $=2.7-3.1$. 
Doleryte - A very hard, crystalline eruptive rock. Its color is always dark, from grayish and bluish to greenish-black and brownish-black. It is a crystalline variety of basalt, and consists of labradorite, augite, olivine, and oftens contains the minerals magnetite and apatite. G. $=2.75-3.1$. Silica $50-55$ p. c. Granitoid to aphantic in texture.

Basalt-A compact, minutely crystalline mixture of labradorite and augite, with olivine, magnetite and titanite. The olivine occurs in the base like green bottle glass. Basalt is very hard and of various shades and colors, from gray to black. It is a very common eruptive rock, filling volcanic vents, fissures, and occurring in vast sheets covering large areas of country. Basalt is often vesicular and assumes many columnar forms.

Diabase-An ancient doleryte or crystalline basalt. It is composed of the minerals labradorite, augite, olivine and chlorite. It is a dark, compact rock, resembling doleryte and basalt, from which it can generally be distinguished by the presence of light-green patches of chlorite, arising from the decomposition of the olivine constituent. No glass in base.

Silica 53 p. c. Alumina, about 20 p. c.

Breccia-A rock formed out of the angular fragments ejected from volcanoes. It is of frequent occurrence in lava-flows. Some are of sedimentary origin (Dana). Tufa-A similar rock and of like origin, but with the fragments smaller. It is a fine sand conglomerate.

Mica-schist-A foliated arrangement of quartz and mica; probably a schistose greisen-granite, or dioryte. The mica probably derived from the decomposition of 
the feldspar. It is generally associated with archæn rocks (metamorphic).

Chlorite-schist-Another metamorphic rock, consisting of a foliated arrangement of quartz and chlorite, containing also magnitite and mica. This rock is greenish in color and has a greasy feel. It is generally associated with gneiss.

Hornblende-Schist-A foliated arrangement of quartz and hormblende, sometimes with orthoclase. It is dark-greenish in color, and is a schistose structure of amphibole or massive hornblende.

Talcose-schist-An arrangement of quartz and talc in layers. It is light-green in color, very greasy to the touch, and occurs only in isolated beds. Metamorphic.

Hydromica-schist-Commonly called talcose-schist. It is a choritic-mica schist with water.

Soapstone or Steatyte-A highly compressed, schistose, massive talc, often impure; color, grayish-green, gray and white. Easily cuts with a knife. Metamorphic.

Serpentine-A yellow, greenish-yellow, or green mottled rock, greasy to the touch and easily scratched with a knife. It is a result of the decomposition of olivine-bearing schists, the silicate of magnesia contained in the olivine rock having become hydrated ( $i$. e., watered or moistened). Metamorphic.

STRATIFIED, SEDIMENTARY, OR AQUEOUS ROCKS.

Silt-A fine sediment which gathers in quiet waters, in hollow places of rivers, lakes, estuaries and seas.

Alluvium, Silt, Till-Alluvium is the earthy deposit made by running streams or lakes, especially dur- 
ing times of flood. Silt is the same material deposited in bays and harbors, where it forms the muddy bottoms and shores. Till is the unstratified sand, gravel and stones, with more or less clay, deposited by glaciers. Called also unstratified drift.

Detritus is a general term applied to earth, sand, alluvium, silt, gravel, because the material is derived, to a great extent, from the wear of rocks through disintegrating agencies, mutual attrition in running water, and other methods (Dana).

Clay-An exceedingly fine-grained, soft, moist rock, formed of minute particles. It is the result of the decay of various aluminious silicates, always containing water. When quite pure is is white, but generally colored red, blue, green gray, brown, etc., from the presence of various impurities.

Marl-A general term used for all compounds of lime and clay. When clay predominates they are called clay marls; when lime is in excess, lime marls. They are compact rocks, breaking with a conchoidal fracture. They are of various color, from liver-brown and red red chiefly, and often contains nodules of limestone.

Mudstone-Massive, consolidated clay. It does not split into layers or laminæ.

Shale-A consolidated clay which splits into thin parallel laminæ, which indicates various cessations and directions of the original deposition. Shale was probably deposited as silt in the beds of rivers, lakes, estuaries and seas. It is of various colors and shades, and often contains fossils.

Slate-A hard, consolidated shale. It splits off into laminæ, which have, however, nothing to do with 
the original planes of deposit, but are the result of cleavage. Color, gray, blue, green, purple, and somtimes black. Roofing slate is a compact kind which splits into very fine and even sheets.

Argillyte - A slate in which more or less mica is present. The flakes of the mica occur in layers along the cleavage planes, a result of metamorphism.

Sandstone-Consolidated sand. It bears the same relation to sand as conglomerate does to gravel, and is the result of cementing action. Sandstones are composed principally or wholly of the mineral quartz. 'They are of various colors. Calcareous sandstone is a variety containing lime of a gray to white color.

Conglomerate-Is gravel consolidated into a compact mass, made up in part of rounded pebbles cemented together.

Grit-A variety of sandstone more common in the older than in the later formations. It is composed of coarse, angular grains of quartz, which point to its arrangement in strata and consolidation in stone within a short space of time after its separation from its parent rock.

Quartzyte - A compact, exceedingly hard rock, composed of granular quartz. It is a metamorphic sandstone, and it occurs in interstratified beds.

Flagstone-A sandy-slate, or a slaty-sandstone.

Loess-A sandy, light-colored clay. It is dry and compact.

Till-A glacial-age deposit of boulders, clay, etc.

Fuller's-earth-A fine-grained argillaceous powder, when pulverized. 
Tripoli-earth-A powdery rock, formed of minute frustules of diatom plants.

Limestone-A grayish, yellowish or brownish rock of various degrees of purity. It is, when pure, formed of calcium carbonate which has been precipitated from water holding lime in solution. $\mathrm{H} .=3 . \mathrm{G} .=2.25-2.75$.

Stalactites-These are pendent, and stalagmites are upright, limestone. They may be of any size, from a mere thread up to a solid pillar many feet in length and diameter. They are the result of dripping lime water.

Marble-A granular or crystalline limestone, due to metamorphism. Of various colors, from white to gray, with reddish and other tints. Impurities are mica, tremolite, pyroxene, scapolite, pyrite, serpentine, chlorite, spinel, graphite, etc. Varieties-Calcite, Dolomite and Calcite-Dolomite Marble.

Calcareous-tufa (travertine)-A lime-carbonate deposit, formed by springs issuing from limestone and it is a sediment precipitated from their waters.

Hydraulic limestone-Contains a small portion of clay and has the property of hardening under water after being calcined or burnt.

Dolomyte (magnesium limestone) - A dirty grayish or yellowish rock. When pure, it consists of 54 per cent magnesium-carbonate and 46 per cent. of calcium carbonate. It is compact, but often assumes globular or other concretionary forms. It is harder than limestone and does not effervesce so freely in acids. When metamorphosed it makes an impure marble, frequently showing veins of iron oxide running through the ground mass. 
Quartz-The mineral silica. It often occurs in veins, sheets and dykes, more especially in the older formations. It is of various colors, from pure white to gray, blue and even black.

Siliceous Sinter-A white, gray, light-pink or blue powdery deposit of almost pure silica, which has been deposited from hot geysers and mineral springs.

Crinoidal-limestone-A rock composed of the calcareous remains of crinoids, shells, corals and other marine life.

Chalk-A soft, white, calcareous rock, formed entirely of the crumbled remains of foraminifera and other marine fossils.

Coral-A rock formed of the accumulated remains of the coral insect.

Peat-A dark-brown mass of compressed marshy vegetation. It is used as fuel.

The foregoing description of the more common rocks are aimed to assist the young miner in his work of identification, and to enable him to assign to each kind of rock its proper name wherever met with in nature.

C. W. Moore's "Practical Gnide for Prospectors, Explorers and Miners." 


\section{A GLOSSARY OF MINING TERMS.}

AOEqUiA. A ditch. Spanish.

ADrT. A horizontal drift or other passage used as an opening or drain to a mine; applied to no level except one opening on the surface. Latin.

Adventurer. A shareholder.

Alligator. A rock breaker operating by jaws.

Alluvrum. The sediment of streams and floods. Latin.

AMALGAM. The mechanical combination of quicksilver with gold or silver.

APEX. The top of a vein. Latin.

Arastra. A circular mill for grinding quartz by trituration between stones attached loosely to cross arms. $S p$. port.

ARCH. A part of the gangue left standing for sup-

Argentiferous. Silver-bearing. Lat.

Ascension Theory. That refering the filling of fissures to matter from below. Von Cotta.

Assay. A test of the mineral contained in a larger mass by extracting and weighing the product of a sample.

Assessment Work. The annual labor $(\$ 100)$ required to hold a location.

AтtLle. Waste rock. Cornish.

Auriferous. Gold-bearing. Lat.

ВАСK. The roof of a drift, stope or other working.

Bat. A mine. Corn.

Bank. The surface at the pit's mouth. (2) Dump.

BANKSMAN. The man at the shaft mouth who handles the bucket. Corn. 
BAR Digginas. Gold washing on river-bars.

Barriers. Posts of unworked gangue or coal left to prevent drainage from mine to mine.

Base Bullion. Pig lead containing its gold and silver unseparated.

Base Metals. All metals except gold, silver, mercury and the platinum group, which are termed noble metals.

BED. A horizontal seam or deposit of ore.

BED Rock. The solid rock outcropping at surface or underlying the gravel, slide or other loose earth.

BismutH. A hard, brittle metal of grayish-white color, reddish tinge, used chiefly as an alloy. At. wt. 210 ; symbol $\mathrm{Bi}$.

BLACK JACK. A dark variety of zinc blende, or sphalerite.

BLENDE. A sulphide of zinc.

Blossom. Decomposed outcrop of a vein. Gossan. Iron hat.

Blow-out. A spreading out-crop.

BONANZA. Fair weather at sea; a large body of paying ore. Became a familiar term upon the opening of the immense ore bodies in the Comstock. $S p$.

Boomine. A kind of placer mining where the water is accumulated in a dam and let out at intervals, so as to utilize its cutting power in the form of a torrent.

Bоom Diтcr. The ditch from the dam used in booming. (2) A slight channel cut down a declivity into which is let a sudden head of water intended to cut to bed-rock and prospect for the apex of any underlying lode.

Borraska. The reverse of bonanza. Out of pay.

BOULDER. A large, loose, rounded stone.

Breast. The heading of a drift, tunnel, or other horizontal working.

BRECCIA. A conglomerate of angular fractions.

Britrue Silver. Stephanite. A sulphide of antimony and silver containing 68.5 per cent silver with the antimony variable. Sometimes contains iron, copper 
and arsenic; variable in color, hardness and specific gravity.

Broaching. Trimming or straightening a working.

Buddling. Separating ores by washing.

Bullion. Uncoined gold or silver.

CACHE. A place where a prospector's provisions or outfit is buried or hidden.

CAGE. The frame to hold the bucket or car.

Calamine. An ore of zinc. Lapis Calaminaris.

Canon. A narrow valley. Termed Box Canon when the sides are perpendicular. $S p$.

CAP. Space where the walls contract so as to leave a trace of the vein. A pinch. (2) A space in the vein where the gangue becomes barren.

Carbonates. The combination of carbonic acid with bases. Soft carbonates have lead for a base. Hard carbonates have iron for a base. An ore of lead and silver.

Cement. Gold-bearing gravel united and hardened into a compact mass.

Chaffee Work. A Colorado term for annual labor. Jerome B. Chaffee was Territorial delegate when the Mining Act of 1872 was passed.

CHEEK. The side or wall of a vein.

Chrmney. A pocket or ore body when found pipeshape, with general perpendicular position.

Chlorides. Compounds of chlorine with other elements.

Chute. (or Sноот.) A flume for sliding ore. (2) A chimney of ore. French.

Cinnabar. Sulphide of mercury.

Claim. A location. The amount of ground which may be located by a single person or association.

Clean-uP. The operation of collecting the gold which has settled in the flume of a placer or in an arastra.

Cleavage. The property of splitting more or less readily in certain definite directions. 
CoAster. One who picks dump, or gleans in abandoned mines for ore in sight.

Cobbing. Ore sorting.

Collar. The top of a shaft or winze. (2) The timbering of a shaft when carried above the surrounding surface.

Color. A particle of gold in the pan.

Concentration. The removal by mechanical means of ore from the gangue or slime.

Contact. The plane of meeting of two formations.

Contact VEIN. A vein along the plane of contact of two dissimilar formations, consequently separating the two formations. Von Cotta.

Copper. A metallic element; red; fusing point 1996 deg. Fahr. Symbol $C u$. Atomic weight 63.5. Specific gravity 8.9 .

CosT-Book Company. A system of mining partnership local to Cornwall and Devon.

Country Rock. The rock beyond the sides of a lode. The strata between or across which the lode is found.

Course of Vein. Its strike. The horizontal line on which it cuts the country rock.

CoуотікG. Spasmodic, irregular surface mining. gold.

Cradle. A rocker. A short trough for washing

Cribbing. The timber lining of a drift, shaft, winze or mill-hole. The term is also applied to rough or light timbering as distinguished from solid set work.

Cross Course. An intersecting vein.

Cross Cut. A level driven across the course of a vein. A short tunnel.

Cut. To intersect a vein. OPen Cut. A horizontal opening at the surface not reaching cover.

Cyanide. A compound of cyanogen with a nietal. The Cyanide Process of gold extraction is performed by passing an auriferous solution of potassium cyanide over zinc shavings, by which the values are precipitated.Henry Lewis on Gold Mining. Thos. R. Beaumont. 
Dead Riches. Base bullion.

DEAD Work. The developing of a mine preparatory to stoping.

DEBRIS. The loose fragments detached from the bed rock and washed down, to which the term slide is more appropriate; waste rock of any kind. French

DEEP. The lower portion of a vein.

Denouncement. The Mexican or Spanish equivalent to "location and record" of a claim.

Descension Theory. The theory that veins were filled from above.

Digangs. Placers. Amer.

DIKe, oR DYKe. A fissure made and filled by plutonic action. Its rock is most commonly porphyry. It is often barren, but in some cases mineralized; or may carry a mineralized selvage and so appear as the wall of a lode.

Diluvium. A deposit of loose boulders, earth. etc., attributed, geologicaly, to deposition from water.

Dip. The line of declination of strata-Bainbridge. Yale-The angle which a lode makes with the plane of the horizon. Von Cotta-The departure of a vein from the perpendicular or from the horizontal.

Diтсн. An artificial watercourse, flume or canal, with or without natural channels.

Divining RoD. A stick of witch-hazel or other like device used in prospecting for lodes. Law v. Grant, 7 M. R., $5 \%$.

Doliar. From the German Thaler. 100 cents. GoLD. 23.22 grains, alloy 2.58 grains, weight 25.8 grains; coined 1849-1902. SILver. 3711/4 grains, alloy $411 / 4$ grains, weight $4121 / 2$ grains; coined 1794 1804, 1836-1838, 1840-1873, 1878-1902. Legal tender unlimited. The Mexican dollar contains 37\%.17 grains silver and 40.62 grains alloy. Spanish dollar the same. E. O. Leech, Director U.S. Mint.

DRIFT. An underground passage driven horizontally on, or with, the vein. 
DownCAst. A ventilating shaft with descending current of air.

Dомг. A deposit, or place of deposit, of waste rock or tailings.

Ervan Course. A plutonic dyke. Lyell. Argall. Corn.

EYE. The top of a shaft.

FACE. Synonymous with breast.

Fатном. A space 6 feet forward and 6 feet vertical with the width of the vein. Corn.

FAULT. A dislocation of the strata. Bainbridge. Yale. The dislocation of a vein from its original position; a heave; a throw. Von Cotta.

FEEDER. A small vein starting from some distant point and running into a main lode. It is practically synonymous with spur. See Bainbridge.

Fendspar. A vitreous crystalline constituent of granite, gneiss, porphyry and many other rocks.

Fissure Vein. A fissure or crack in the zarth across its strata, filled with mineralized mater.

Float. Loose quartz detached from the vein and found below it.

Float Ore. Masses or particles of ore detached from the vein and found below it.

Flookan. A soft, decomposed cross-course. Cornish.

FLoor. The rock underlying a horizontal vein or deposit.

FLUME: A ditch carried in frame work on or above the surface.

Fоот Wall. The under wall of the vein.

Forfeiture. The loss of possessory title as the result of abandonment or the failure to comply with the conditions under which the title was held.

GAD. A small pointed wedge.

Galena. A sulphide of lead. When not amorphous, is crystallized on the cubic system; when pure contains 86.6 per cent lead, 13.4 per cent sulphur. Carries silver in greatly varying quantities. 
Gallery. A level or drift; applied chiefly to colliẻries.

Gangue. Crevice material; vein matter; the base material forming the matrix of the ore.

Gasir VEIN. A vein which continues for practical purposes only a short distance below the sod, generally narrowing as it descends.

GEoDE. A rounded nodule of stone, containing a cavity studded with crystals or mineral matter; the cavity in such nodule.

GNeiss. A rock composed of the same constituents as granite, but foliated or stratified.

GoB Fine. Fire in collieries produced by spontaneous combustion.

GoLD. A metallic element; bright yellow; specific gravity, 19.34 ; fusing point, 2016 degrees Fahr. Symbol, $A u$. Atomic weight, 196.6. One ounce pure gold coined in U. S. dollars is worth $\$ 20.6 \%$.

Gossan. See Iron Hat.

Govge. A soft selvage; a clay streak found following a wall, or a slip or an ore measure.

Grass. The surface over a mine. Corn.

Grass Roots. A term used where a working is started from, or worked up to, the surface. Amer.

Granite. A plutonic crystalline rock, composed of feldspar, quartz and mica.

Gray Copper. Tetrahedrite. An ore containing copper 15 to 42 per cent, combined with iron, zinc, silver, mercury, arsenic and antimony. It varies in color, hardness and specific gravity.

Grub Stake. Provisioning a prospector on a bargain to share his discoveries.

Hanging WaLl. The upper wall of a vein.

HeAding. The breast or face of a working.

Headings. The mass of gravel and pay dirt above the head of a sluice. 
Heave. The horizontal dislocation of a lode.

High Explosives. Those of greater detonating force than black powder.

Horse. A mass of country rock between the enclosing walls of a vein. To constitute a Horse, "It is necessary that the walls should converge about the mass below and at both ends, but the greatest known horses do not converge over head. The two walls coming to the surface are in some instances 1,000 feet apart." Testimony of Clarence King in the Dives Case.

HUDGE. An iron bucket for hoisting.

Hrdraulics. That method of placer mining where the gravel is washed by a stream operating under hydraulic pressure.

HUNGRY. Barren.

Impregnation. A metallic deposit having undetermined limits in no way sharply defined. Von Cotta.

InCline Drift. A drift run at an incline to subserve the drainage. (2) A misnomer applied to a slope sunk upon a deposit having slight departure from the horizontal.

Infiltration Theory. That which refers the origin of the ore to the deposit of mineral from water holding it in solution.

InJECTION THEORY. That which refers the origin of the ore to the introduction of igneous fluid.

In Place. In Situ. In words used in Section 2329 of the U. S. Revised Statutes, qualifying the words "quartz or other rock," and to distinguish lode from placer claims.

Iron Hat. (Eisen Hut.) The outcrop of a lode, it being usually colored by the decomposition of the iron. German. Von Cotta.

JIG. A machine for concentrating ore by means of sieves. Corn.

JUMP. To take forcible possession of a claim.

To relocate abandoned property.

KIBbLe. A kind of hoisting bucket. 
Lagaing. Poles or small timbers used for spanning from one stull-piece to another, for cribbing millholes and for lining behind the timbers of a shaft.

LEAD. An objectionable form of the word lode.

LEAD. A metallic element; bluish-white; fusing point, 617 deg. Fahr. Symbol, $\mathrm{Pb}$. Atomic weight, 20\%. Specific gravity 11.30. Galena and carbonates are its most common ores.

Ledae. A term in use on the Pacific Slope, synonymous with lode.

LENGTH. A certain portion of a vein when taken on a horizontal line on its course.

LEVEL. A drift along the vein; the word generally used where there are a series of drifts, as first level, second level, etc.

LIFT. The space between two levels.

Litrle Grant. A jointed iron pipe and nozzle decreasing in diameter with the increase of the hydraulic pressure; used in placer mining.

LOcATION. Those successive acts by which a claim is appropriated. (2) The claim itself. Amer.

Lode. An aggregation of mineral matter containing ores in fissures. Von Cotta. A vein of metallic ore. A ledge. A fault in the country which has become mineralized. A. H. Green.

MaN HoLt. An opening just large enough to permit access between two workings.

Matrix. (Of the lode.) The country rock in which the vein is found. (Of the ore.) The rock or earthy material enclosing the ore; the vein-stone. Latin.

Mercury. Quicksilver. A shining silver-white metal, liquid at temperatures above $-40 \mathrm{deg}$. Fahr. Sp. gr., 13.5. At. wt., 199.7. Boils at 669 deg. Fahr. Symbol, $\mathrm{Hg}$.

Metallurgy. The art of working metals, including smelting, refining, and parting them from the ores.

Mica. One of the constituents of granite. When separately crystallized is found in clear, laminated 
plates. Found in the lode, as well as in the matrix of the lode.

Milu Hole. A passage left in the stope for throwing down rock and ore.

Mrul-Run. The returns of a lot of ore; the assay of ore in quantity as distinguished from a specimen assay.

Mrne. Any excavation made for mineral which can be profitably operated. (2) An open as distinguished from an untouched deposit. (3) Underground as distinguished from superficial workings or quarries.

MineR's INCH. There is an attempted statutory definition in Colorado M. A. S. Sec. 4643 which is obscure and inexact. See also 246\%. Orifices constructed as this statute directs, will deliver through each square inch of opening, a quantity which varies from 1.4 to 1.7 cubic feet of water per minute. The custom among engineers is to take 1.5 cubic feet of water per minute as the equivalent of an inch. For Illustration: 1,000 miner's inches of water (25 cubic feet per second) is about the quantity which would be carried by an ordinary wooden flume 30 inches wide, flowing 18 inches deep, if the flume had a fall of one inch per box of 12 feet ( 36 feet per mile), this giving the stream a velocity of 6.7 feet per second. Edmund B. Kirby.

Miner's Right. The license to locate, used in Australia.

MoYLE. A drill or short har sharpened to a point, used in cutting hitches and in broaching.

Nodule. A small, rounded, stony concretion.

OPEN CUT. A longitudinal surface working not entering cover.

OPERATOR. One who works a mine either as owner or lessee.

ORE. The mechanical or chemical compounds of the metals with baser substances. The conventional divisions in the ore market are: DRY ORE: An ore which does not contain any lead, or less than 5 per cent. ; MiLLING ORE: A dry ore that can be amalgamated or treated 
by leaching and other processes; usually these ores are low grades, free, or nearly so, from base metals. SHIPPING ORE: Such as is better adapted to smelting than any local treatment. Any ore of greater value when broken than the cost of freight and treatment. REFRACTORY ORES: An ore containing in quantities zinc, arsenic, antimony or other base metals, which prevent economical treatment by usual and available processes. $W$. J. Chamberlain \& Co.

Ore Reserves. The ore body where exposed ready for stoping.

Outcrop. That portion of a vein appearing at the surface.

Output. The gross product of a mine.

PAN. An iron basin used in gold prospecting.

Patch. A small placer claim outside of the main gulch.

Patio. A yard or court. The space where ore is mixed and amalgmated by tread of horses. $S p$.

Patio Process. The Mexican method of amalgamation of silver ores.

PAY Rock. The lode material in which the mineral or pay is found. See Quartz.

PAY StREAK. The ore body proper, or the seam of decomposed material which takes its place and preserves the continuity of the ore body.

Pent House. A shed or horizontal barricade across one end of a shaft, made of strong timbers loaded with rock to protect against any accidental fall from above. Corn.

Phonolite. A greyish, compact, felspathic rock yielding a metallic sound under the hammer; clinkstone.

Pinch. The narrow space where the walls come close together.

PIT. A shallow shaft.

Pitch. The dip of a lode.

Placer. A deposit of gold not in place; applied to all classes of gold deposit, including cement and 
channel claims, except lodes in place. For special meaning under the law, see Section 2329 U. S. Rev. St.

Plat. A small chamber on the side or sole of a level where it intersects a shaft, made to facilitate dumping. Where it is cut in the sole it is called a tripplat. Corn.

Pocket. A detached ore body; a nest of ore.

Pockery. A term applied to a mine where the pay ore occurs in small detached bodies with intervals of poor ore or barren material. The word implies a slur on the mine. Paull v. Halferty, 9 M. R. 149.

Porphyry. A general term including such plutonic rocks as exhibit well-formed crystals, usually of feldspar, in a finely granular or compact base of the same. $G r$.

Porphyritic Granite. A base of granite containing prominent crystals of feldspar.

Prospecting. A search for deposits, applied both to the seeking of undiscovered veins and to the investigation of the value of known veins by exploration.

Pyrites. (White) A sulphide of iron. (Yellow) A sulphide of copper. A bright, crystallized, metallic-looking and very common gold-bearing ore, usually low-grade and spoken of in common parlance as the "Iron." Gr.

QUARRY. Any open work in rock on a plan of excavating the entire mass, as distinguished from working a seam or vein by shafts or aproaches under cover.

Quartz. Silica. A constituent of granite. The free gold of California being found in quartz, the word was applied to the gangue of such lodes and so to other forms of vein matter, until it is now used vaguely to mean the ore, the float, the gangue, or that part of the gangue which indicates the pay streak. In the Acts of Congress it is used with the word rock (quartz or other rock) in the sense of pay rock.

QuARTzite. A metamorphosed sandstone. A rock containing usually about 98 per cent. silica, with a small percentage of foreign materials, principally iron. 
Quicksilver. See Mercury.

RAISE. A shaft or winze which has been worked from below.

RHYolyte. A name common to igneous rocks of a wavy texture, indicative of movement or flowing when in a fluid state.

Riffle Blocks. Cross sections of timber set on the floor of a sluice, with irregular spaces between, in which the gold settles. American.

Rist. See Raise.

ReEF. An Australian term for lode or ledge.

Roв. To gut a mine; to work for the ore in sight without regard to supports, reserves or any other future considerations.

Rocker. See Cradle.

Roof. The stratum or rock overlying a deposit, or flat vein.

Royality. The dues to the lessor.

Rustr. Oxidized. Ore coated with oxide. Applies to gold which will not easily amalgamate:

Scale. A loosened fragment of rock threatening to break off and fall.

ScHist. Crystalline, or metamorphic rock with slaty structure; usually carrying mica, sơmetimes argillaceous.

Segregations. All those aggregations of ore having irregular form but definite limits. They differ from beds and lodes by the irregularity of their form; from impregnations by their definite limits. Von Cotta.

Selvage. A lining; a gouge; a thin band of clay often found in the vein, upon the wall.

SEx. Portion of ground taken by a tributer.

SHAFT. A pit sunk from the surface; an opening more or less perpendicular sunk on, or sunk to reach, the vein.

SHIFT. A miners turn or spell of work. Webster. Two shifts imply 16 to 20 hours work; three shifts imply 24 hours' work.

Sill. A windlass frame. (2) Rest for posts. 
Silver. A metallic element; the whitest of the metals; specific gravity, 10.53; fusing point, 1873 degrees; symbol, $A g$; atomic weight, 108. One ounce pure silver coined in U. S. dollars is worth $\$ 1.2929$, gold.

Silver Glance. An ore; when pure contains 87 per cent silver and 13 per cent sulphur. Argentite.

SKIP. A square hoisting bucket running in guides or in grooves.

Slickensides. Smooth, polished portions of the wall or of some verticle plane in the lode, caused by friction. It may occur on the ore itself. German.

SLIDE. One kind of fault-the vertical dislocation of a lode.

SLIDE. The mass of loose rock overlying either lode or country.

SlOPE. An opening driven upon the inclination of the vein.

Sluice. A series of boxes set in line and floored with riffle blocks to catch the gold in a placer mine.

Smelting. The reduction of metals from their ores in furnaces. It is a form of the word melt. In smelting, the ore is melted. In .other processes il is roasted.

SoLE. The floor of a horizontal working.

Sollar. Any platform or wooden floor or covering in a working. Corn.

Sougr. A drain. Eng.

Spar. A general term applied to rock with distinct cleavage and lustre.

SPUR. A branch or offshoot from a larger vein.

SPILING. Timbering used in quicksand or loose ground where lathes are driven behind timbers and kept flush with the heading.

STAMPS. Machine for crushing ores by vertical stroke.

STOPE. The working above or below a level where the mass of the ore body is broken. Corn. 
SToping. The act of breaking the ore above or below a level; when done from the back of the drift it is called overhand or back-stoping; when from the sole it is underhand stoping.

Stratum. A bed of rock or earth of any kind. Dana. The plural is strata.

STRIKe. The extension of a lode or deposit on a horizontal line. Von Cotta. Synonymous with trend and course.

Stulls. Cross timbers at the foot of a stope.

Sublimation Theory. That which refers the filling of fissures to material deposited from ascending steam, or by condensation from a gaseous condition.

Sulphide. A chemical union of sulphur with a metal.

Sulphuret. A sulphide. Sulphide is the more recent and approved term.

Somp. The extension of a shaft, forming a pit for the collection of water. Corn.

Sylvanite. A native tellurium; sometimes called graphic tellurium.

Syndicate. An association or council of persons ; in use since the war to designate any combination formed to carry out a large financial enterprise.

TACKLE. The windlass, rope and bucket. Corn.

TaIlings. The refuse discharged from the tail or lower end of a sluice, or washed from any sort of placer working.

Telluridm. A silver-white, brittle substance, generally classed among metals; usually combined with gold, silver, lead and copper. Sp. gr., 6.65. At. wt., 128. Symbol, Te.

TIN. A soft, malleable, white metal. Sp. gr., 7.2. Fusing point, 442 deg. Fahr. At. wt., 11\%.7. Symbol Sn.

Tributers. Miners who work a set, or piece of ground, taking the proceeds as wages, after royalty is deducted, but who work under direction of the owners and hold no possession or title as lessees. 
Trouble. A fault.

TUNNEL. A horizontal excavation starting at the surface and driven across the country for the discovery or working of a lode or lodes.

TUT Work. Work paid for by the foot, as distinguished from tribute work.

UPCAST. A ventilating shaft where the air ascends.

VEIxs. Aggregations of mineral matter in fissures of rocks. Von Cotta, Saw; Bainbridge. The word vein has a broader scope than lode, including nonmetallic beds. It is also applied, in working, to smaller seams threading the greater deposit. See Vena and Veta.

VENA. A small vein or the branches of the veta, or main vein. Span.

VETA. A main vein. Span.

VuG. A cavity in the ore or rock.

WALL. The plane of the country where it touches the side of the vein, when used in reference to lodes. The side of a level or drift, when used with reference to the workings.

WhEAL. A pit or hole in the ground. A mine. The names of most mines in Cornwall are preceded by the word Wheal. Old form Huel. Corn.

WHIM. A machine for raising the bucket by means of a revolving drum.

WHIP. An apparatus for raising the bucket with rope and pulleys, by horse power on a straight drive.

WINzE. A shaft sunk from a level; not necessarily connecting two levels.

Zinc. A metallic element; bluish-white; fusing point, 773 deg. Fahr.; generally found as a sulphide (blende), or as a carbonate (calamine). Atomic weight, 65.2 ; specific gravity, 8.9. Symbol, $Z u$.

Morrison's "Mining Rights." 
COMPLETE OUTFIT FOR TESTING AND ILLUSTRATING THE PROPERTIES OF MINERALS.

\section{FIRST PROPERTY-COMPOSITION.} BLOWPIPE AND WET WAY OUTFIT.

11 Set (3) porcelain dishes.

21 Diamond steel mortar.

$3 \quad 1$ Pair platinum pointed forceps.

41 Pair heavy tip steel forceps.

61 Steel chisel.

7 1 Charcoal borer, club shape.

81 Charcoal borer, with spatula.

$9 \quad 1$ Pair scissors.

101 Platinum holder, with 6 wires.

111 Plattner's blowpipe lamp, with swivel.

121 Charcoal saw.

131 Mattrass holder.

141 Plattner's blowpipe, nickle plated.

151 Platinum tip for same.

161 Steel hammer, with wire handle.

171 Set moulds, stamps and bone ash.

181 Pair nippers.

191 Double lens.

201 Knife.

211 Dropping pipette.

22 1 Camel hair brush.

236 Mattrasses.

241 Glass alcohol lamp.

251 Chamois skin.

266 glass tubes.

$271 / 2$ Doz. charcoals.

28 Coal and ash trays.

292 Books test papers. 
303 Frames, with 61 glass stoppered and labeled reagent bottles, containing the following reagents :

1 Soda Bicarbonate.

2 Powdered Borax Glass.

3 Salt of Phosphorus.

4 Bismuth Flux.

5 Boracic Acid.

6 Boracic Acid Flux.

7 Copper Oxide.

8 Potassium Sulphate.

9 Ammonia Water.

10 Nitric Acid.

11 Hydrochloric Acid. $<$

12 Sulphuric Acid.

13 Potassium Bisulphate.

14 Antimony Teroxide.

15 Antimony Pentoxide.

16 Ammonium Magnesia Mixture.

17 Cyanide of Potassium (Salt).

18 Barium Dichloride.

19 Lead Oxide (Litharge).

20 Hydrogen Peroxide.

21 Tartaric Acid.

22 Ammonium Sulphocyanide.

23 Hydrodisodic Phosphate.

24 Ammonium Carbonate.

25 Ferrous Sulphate.

26 Molybdate Solution.

27 Platinic Chloride.

28 Bichloride of Mercury.

29 Stannic Oxide.

30 Ammonium Sulphide.

31 Metallic Magnesium.

32 Metallic Copper (Test).

33 Metallic Lead (Granulated).

34 Metallic Zinc.

35 Metallic Tin (Test).

36 Metallic Iron (Test). 
37 Powdered Silica.

38 Cobalt Solution.

39 Ammonic Chloride.

40 Sodic Acetate.

41 Sulphretted Hydrogen Water.

42 Silver Nitrate.

43 Potassium Hydrate.

44 Copper Sulphate.

45 Sodium Hydrate.

46 Tin Bichloride.

47 Manganese Dioxide.

48 Calcium Dichloride.

49 Acetic Acid.

50 Potassium Ferrocyanide. ¿

51 Citric Acid.

52 Nitrophenic Acid.

53 Peroxide of Lead.

54 Sodium Chlorate.

55 Magnesia Mixture.

56 Magnesium Sulphate.

57 Sodium Nitrate.

58 Terchloride of Gold.

59 Iron Sesquioxide.

60 Albumen.

61 Potassium Ferricyanide. 2

Packed in a wood carrying case; price.......\$40.00

LUSTER.

\section{SECOND PROPERTY.}

a. Kinds of Luster. 1, Metallic; 2, SubMetallic; 3, Adamantine; 4, Vitreous; 5 , Sub-Vitreous ; 6, Resinous ; 7, Greasy ; 8, Pearly; 9, Metallic-Pearly or Metalloid; 10, Silky; 11, Dull, without Luster. $b$. Degrees of Intensity of Luster. 12, Splendent; 13, Shining; 14, Glistening; 15, Glimmering. Large specimens mounted on cherry blocks..........\$ $\% .50$

b. Same, but specimens smaller and in pasteboard trays ................. 3.75 
DIAPHANEITY.

1, Transparent; 2, Semi-Transparent; 3, Translucent; 4, Sub-Translucent; 5, Opaque. Large specimens mounted on cherry blocks ................

\section{THIRD AND FIFTH PROPERTY.}

Color aND Streak.

a. 25 of the most important specimens mounted on cherry blocks.........\$12.00

b. 56 large specimens, illustrating the metallic and non-metallic colors, mounted on cherry blocks ...............28.00

c. Same as foregoing, but smaller specimens and in pasteboard trays........ 14.00

\section{FOURTH PROPERTY.}

Scale of HaRdNess.

a. 1, Talc; 2, Gypsum; 3, Calcite ; 4, Fluorite; 5, Apatite; 6, Orthoclase; 7, Quartz; 8, Topaz; 9, Corundum; 10, Diamond.

With streak plate and file. Tray......\$ 5.00

b. Same, but smaller specimens, in hard wood case .................. 1.50

c. Same as foregoing, without diamond.... 1.00

Fracture.

\section{SIXTH PROPERTY.}

1, Even; 2, Uneven; 3, Conchoidal ; 4, SubConchoidal ; 5, Splintery; 6, Hackly. Large specimens mounted on cherry blocks

Clemavage.

1, Cubic ; 2, Octahedral ; 3, Rhombohedral; 4, Basal; 5, Prismatic. Large specimens mounted on cherry blocks....... SEVENTH PROPERTY.

TENACITY.

1, Brittle; 2, Sectile; 3, Malleable; 4, Flexible; 5, Elastic. Large specimens mounted on cherry blocks........ \$2.50 


\section{EIGHTH PROPERTY.}

Crystal System and Forms.

100 white crystal models in Plaster.....\$16.00

\section{NIN'TH PROPERTY.}

Scale of Fusibility.

a. 1, Stibnite; 2, Natrolite; 3, Almandite; 4, Actinolite; 5, Orthoclase; 6, Bronzite. In paste board trays.........\$1.00

b. Large specimens, mounted on cherry blocks

TENTH PROPERTY.

Specific Gravity. (G.)

Prof. Jolly's Spiral Balance, for rapid and exact determination of the specific gravity of minerals, with 4 assorted spirals, on wooden support and scale, on mirror glass; Price ...............\$17.00

COLLECTION OF MINERALS, MODELS, BI.OWPIPE AND ASSAY OUTFITS.

Blowpipe Collection.

25 specimens, in hard wood case......\$ .75

50 specimens, in hardwood case....... 1.50

100 specimens, in hard wood case....... 3.50

200 specimens, in hard wood case...... 8.00

Ores and Metallic Minerals.

25 specimens, only the common metals, averaging $3 / 4 \times 3 / 4$ inch, case........\$ 1.00

25 specimens, larger size, printed labels, in pasteboard trays .............. 2.50

50 specimens, illustrating ores of both the common and rare metals, printed labels, in pasteboard trays ............. 10.00

Ores of the Rarer Metals.

15 specimens $\ldots \ldots \ldots \ldots \ldots \ldots \ldots \ldots \ldots \$ 4.50$

25 specimens $\ldots \ldots \ldots \ldots \ldots \ldots \ldots \ldots \ldots .10 .00$

Gold and Silver ORes.

25 specimens .............. \$ 6.00 
Copper Ores.

15 specimens $\ldots \ldots \ldots \ldots \ldots \ldots \ldots \ldots .3 .00$

25 specimens $\ldots \ldots \ldots \ldots \ldots \ldots \ldots \ldots \ldots .6 .60$

Iron Ores and Minerals.

25 specimens ................ 6.00

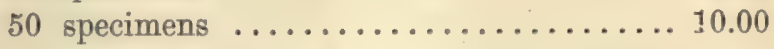

Lead Ores and Minerals.

15 specimens $\ldots \ldots \ldots \ldots \ldots \ldots \ldots \ldots \ldots . \ldots 3.00$

25 specimens ................. 6.50

50 specimens $\ldots \ldots \ldots \ldots \ldots \ldots \ldots \ldots .25 .00$

Zinc Ores and Minerals.

15 specimens ................. \$ 400

25 specimens $\ldots \ldots \ldots \ldots \ldots \ldots \ldots \ldots \ldots$

Crystal Models, Preston's, of Celluloid.

A new and most excellent invention is Preston's set of six crystal models. They are made of transparent celluloid and average 4 inches longest diameter. They exhibit the six different systems of crystallization, and show various derivative forms by means of internal crystals, also of celluloid. The axes of the crystals are shown by various colored silk threads, the same axis by the same color, different axes by different colors. Set of six in well made case ................\$16.00

Crystal Models, of Hard Wood.

34 models, with reference list, in box....\$10.00

50 models, with reference list, in box.... 15.00

108 models, with reference list, in box.... 24.00

Crystal Models, of Glass.

21 cut glass models, showing the crystallographic forms and natural colors, of uncut gems, in case ...........\$18.00

Crystal Models in Plaster.

100 white models ..............\$16.00

50 colored faced models, mounted in brass holders and again on hard wood blocks, 
label giving name of minerals and crystallographic formula .......... 15.0 100 colored faced models . . . . . . . . 30.00 200 colored faced models .......... 65.00

BLOWPIPE APPARATUS, AS DESCRIBED IN "BROWN'S MANUAL OF ASSAYING."

11 Set (3) porcelain dishes.

21 Diamond steel mortar.

$3 \quad 1$ Pair platinum pointed forceps.

41 Pair steel forceps.

$6 \quad 1$ Steel chisel.

7 1 Charcoal borer, club shape.

81 Charcoal borer, with spatula.

91 Pair scissors.

101 Platinum holder, with 6 wires.

111 Plattner's blow-pipe lamp with swivel.

121 Charcoal saw.

131 Matrass holder.

141 Plattner's blowpipe, nickel plated.

151 Platinum tip for same.

161 Steel hammer, with wire handle.

171 Set moulds and stamps.

181 Pair nippers.

191 Double lens.

201 Knife.

211 Dropping pipette.

221 Camel hair brush.

236 Matrasses.

241 Glass alcohol lamp, with metal top.

251 Chamois skin.

$26 \quad 6 \mathrm{Gl}$ רss tubes.

$27 \quad 1 / 2$ Doz. charcoals.

28 Coal and ash trays.

292 Books test papers.

30 Frame, with 18 glass stoppered and labeled reagent bottles, containing the following re. agents : 
Tin. (Test) Carbonate soda.

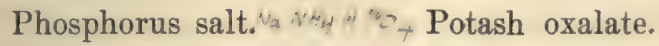

Borax powder.

Salt.

Borax glass.

Boracic acid, fused.

Soda nitrate.

Boracic acid, cryst.

Charcoal.

Plattner's flux.

Bismuth flux.

Bone ash, sieved.

Test lead.

Bone ash, washed.

Copper oxide.

Price for complete set securely packed in neat

wooden carrying case ............ \$30.00

ASSAY OUTEIT FOR PROSPECTORS.

1 Portable Button Balance and weights.

1 Pulp balance and weights.

1 Furnace ("Jackass").

2 Muffles.

200 Scorifiers.

50 Crucibles.

1 Quart mortar and pestle (iron).

2 Pair tongs.

1 Magnifying lens.

1 Lead mould.

1 Cupel mould.

1 Magnet.

3 Pairs pliers.

1 Spatula.

Glass rod and tubes.

1 Glass alcohol lamp.

1 Sieve, 60 mesh.

3 Beakers and covers.

1 Blowpipe, Plattner's.

3 Funnels.

1 Package filter paper.

1 Button brush.

1 Wash bottle.

6 Parting flasks.

1 Tripod.

6 Annealing cups. 
2 Hammers.

4 Lbs. litharge.

5 Lbs. soda bicarb.

1 Lb. argols.

1 Lb. muriatic acid, c. p.

1 Lb. nitric acid, c. p.

10 Lbs. bone ash.

2 Lbs. borax glass.

1/4 Oz. silver foil, c. p.

$1 / 2$ Lb. rolled lead, c. p.

10 Lbs. granulated lead, c. p.

1 Pint alcohol.

2 Lbs. lead flux.

Boxing and cartage, complete, f. o. b., Denver. .\$12500

Price's given are according to the Denver Fire Clay Company's catalogue 


\section{INDEX TO MINERALS}

Actinolite ...........132

Aeschynite ..........117

Agate ...............129

Aikinite ............104

Alaskaite ............104

Almandine ..........118

Albertite ............127

Albite ..............136

Allophane ..........145

Altaite .............103

Alunite .............119

Alunogen ...........119

Amber .............126

Amblygonite ..........119

Amethyst .........129

Amethyst, Oriental ...118

Amphibole ..........132

Analcite ............142

Andalusite ..........139

Andesite ...........136

Angelsite .........1C5

Anhydrite ...........121

Anorthite ...........136

Anthracite .........127

Antimony, Native ...125

Apatite ...........121

Apophyllite ..........144

Aragonite ...........121

Argentite .......... 97

Arsenic, Native .......124

Arsenolite ............125

Arsenopyrite ........111

Asbestus ............132

Asbolite ...........109

Asphaltum ..........126

Astrophylite ........137

Atacamite ...........101

Aurichalcite ........107

Autunite ............115

Aventurine ..........129

Axinite .............133

Azurite .............102
Balas Ruby .........118

Barite ..............122

Beryl ..............131

Biotite ............138

Bismuth, Native .....125

Bituminous Coal .....126

Bloodstone .........130

Boracite .............120

Borax .............123

Bornite ............100

Breithauptite ........109

Brochantite .........102

Bromyrite .......... 99

Brongniardite ........998

Brown Coal ..........127

Brucite ...........120

Caking Coal ........127

Calamine ...........108

Calaverite ..........996

Calcite .............121

Calomel ............110

Cannel Coal ..........127

Carnelian ............129

Cassiterite .........114

Cat's Eye ...........129

Celestite ...........122

Cerargyrite .........999

Cerussite ...........106

Chabazite ...........142

Chalcanthite ........101

Chalcedony ..........129

Chalcocite ...........100

Chalk .............121

Chalcopyrite .......100

Chloritoid ...........141

Chondrodite ........138

Chromite ...........112

Chrysoberyl .........118

Chrysocolla ... ......103

Chrysolite ...........133

Chrysoprase ... ....129

Cinnabar ..........110 
Clausthalite .........103

Cobaltite ..........108

Coal, Anthracite .....126

Coal, Bituminous ....126

Coal, Brown ........127

Coal, Caking ........126

Coal, Cannel ........126

Colemanite .........121

Coloradoite ..........110

Columbite ..........112

Copper, Native ......100

Corundum ..........117

Covelylite ..........100

Crocoite ..........105

Crookesite ..........100

Cryolite ...........118

Cryophyllite ........137

Cuprite .............101

Cyanite ............139

Cyanotrichite .......102

Danburite ...........133

Datolite ...........140

Descloizite .........106

Deweylite ...........144

Diamond ..........125

Dioptase ............103

Dolomite ...........121

Domeykite ..........1 0

Dysluite ...........118

Eggonite ..........114

Elaterite ...........126

Eliasite ............114

Embolite ........... 99

Emerald, Oriental ....118

Enargite ...........101

Enstatite ...........131

Eosphorite ...........119

Epidote.... . . . . . . . 134

Epsomite ..........120

Erythryte .............109

Eucairite ..........997

Euchroite ..........102

Euclase .............138

Euxenite ............116

Fahlunite ..........145

False Topaz .........129

Fergusonite .........116
Ferruginous Quartz ...129

Fibrolite ............139

Fire Opal ............130

Flint ..............129

Fluorite ............120

Franklinite ..........108

Freieslebenite ......998

Galena .............103

Garnet ............134

Genthite ...........109

Gerhardtite ........102

Gersdorffite .........109

Gibbsite ...........118

Gilsonite .......... 127

Glauconite ..........143

Gold, Native ........ 96

Goslarite ..........107

Gothite .............112

Grahamite .........126

Graphite ............125

Greenockite .........114

Grunauite ...........109

Guitermanite ........104

Gummite ...........114

Gypsum ............120

Halite .............122

Iarmotome ...........142

Hatchettite ..........126

Hatchettolite ........115

Hematite ...........111

Hessite ........... 97

Heulandite .........142

Hisingerite .........131

Hornblende .... . . ...132

Hubnerite ..........115

Hyacinth .........134

Hydrophane .........130

Iodyrite . . . . . . . . .999

Iolite ... . . . . . . . . 133

Iron, Native ........... 111

Jamesonite .........104

Jargon ... ..........134

Jasper . . . . . . . . . 130

Jet ... . ..........127

Kaolinite ............145

Kobellite ..........104

Krennerite .........96 96 
Labradorite ........136

Laumontite ... . . . . 144

Lazulite ... . . . . . . 119

Opal .............137

Opal, Precious .......130

Lead, Native .........103

Lepidolite ..........137

Leucite ............135

Leucopyrite .........111

Limonite ...........112

Linarite ...........105

Linnaeite ...........108

Livingstonite ........125

Magnesite ..........120

Magnetite ...........111

Magnolite ..........110

Malachite ..........102

Mallardite ..........113

Manganosite ... ..,...113

Marcasite ...........111

Margarite ...........141

Melaconite ..........101

Melanochroite .......105

Melanterite .........112

Melonite ... .......109

Menaccanite .........111

Menuspite ...........105

Mercury, Native .....101

Miargyrite ..........98

Microcline ..........136

Milky Quartz ........129

Millerite ..........108

Opal, Fire ..........130

Opal, Wood ..........130

Oriental Amethyst ...117

Oriental Emerald ......117

Oriental Ruby ........117

Oriental Topaz .......117

Orpiment ..........124

Orthoclase ..........136

Ozocerite ...........126

Paragonite ..........137

Pectolite ............144

Penninite .......... 140

Periclasite ...........120

Petalite ............132

Petzite ............ 96

Phologopite .........137

Pinite ............145

Plasma ... . . . . . . 129

Plumbogummite .....105

Polyargyrite .... .... 98

Polybasite ..........998

Prase .............129

Precious Opal .......130

Prehnite ...........145

Prochlorite ..........141

Proustite ........... 98

Psilomelane ........113

Pyargyrite ..........97

Mimetite ..........106

Minium ...........104

Mirabilite ..........123

Molybdenite .........124

Monabite ...........117

Muscovite .........138

Nagyagite ..........996

Natrolite ........... 142

Natron ... .........123

Nephelite . .........135

Niccolite ...........108

Nitratine ... .......123

Nitre ..............123

Oligoclase ..........136

Olivenite ...........102

Onofrite ..........110

Pyrite .............111

Pyrolusite ...........113

Pyromorphite ......106

Pyrophyllite ........143

Pyrosclerite .........141

Pyrostilpnite ........ 99

Pyroxene ...........131

Pyrrhotite ..........111

Quartz ............129

Quartz, Crystal ......129

Quartz, Ferriginous ...129

Quartz, Milky ........128

Quartz, Rose .........129

Quartz, Smoky .......129

Realgar ...........124

Rhodochrosite .......113

Onyx ............129 Rhodonite .........131 
Ripidolite .........140

Rock Crystal ........129

Rose Quartz ........129

Rubicelle ...........118

Ruby, Balas ..........118

Ruby, Oriental .......117

Ruby, Spinel .........118

Rutile ............114

Salmiak ............. 123

Samarskite .. .......116

Saponite ... ........144

Sapphire ...........117

Sard ...............129

Sassolite ...........124

Scheelite ...........115

Schirmerite ........ 99

Sellaite ...........120

Semiopal ...........130

Sepiolite ............143

Serpentine ..........143

Siderite ............ 112

Silicified Wood ........130

Silver, Native .......997

Sipylite ...........116

Smaltite ...........108

Smithsonite ........107

Smoky Quartz ........129

Sodalite ...........135

Sphalerite .........107

Spinel .............118

Spinel Ruby ..........118

Spodumene .........131

Stannite ...........114

Staurolite .......... 139

Stephanite ......... 98

Sternbergite ........ 97

Stibnite ............125

Stilbite ...........142

Stoizite ........... 106

Stromeyerite .......997

Strontianite .......... 122

Sulphur, Native .......124

Sylvanite ..........996

Sylvite ............122

Talc ................143

Tellurite ...........124
Tellurium, Native ....124

Tennantite .........101

Tetradymite .........125

Tetrahedrite .........101

Thompsonite ........ 143

Tiemannite ... ......110

Titanite .............140

Topaz ............. 139

Topaz, Oriental ......117

Tobernite ..........115

Touchstone .........130

Tourmaline ..........139

Tremolite ...........132

Tridymite ...........130

Triphylite ...........113

Triplite ...........113

Tripolite ............130

Turquois ........... 119

Ulexite ............121

Ultramarine .........135

Uraninite ..........115

Valentinite ...........125

Vanadinite .........106

Vauyuelinite ........105

Vermiculite ..........141

Vesuvianite ..........134

Vivianite ..........112

Wavellite ...........119

Wad ...............113

Wernerite ...........135

Willemite ...........107

Witherite ...........122

Wolframite .....112, 115

Wollastonite ........131

Wood Opal ..........130

Wood, Silicified .......130

Wulfenite ...........106

Wurtzite ...........107

Xenotime .........116

Yttrocerite .........116

Zaratite ...........109

Zinc Bloom .........107

Zincite ...........107

Zinkenite ..........104

Zircon .............134

Zossite ............133 



\section{The Denver Fire Clay Co. DENVER, COLORADO.}

Manufacturers of

Muffles

Crucibles

Scorifiers

Furnaces

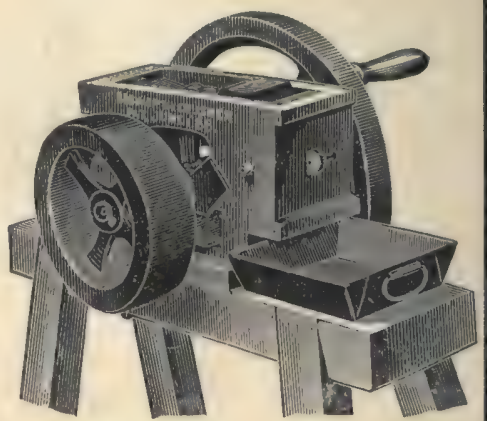

and all kinds of

FIRE CLAY BRICK AND TILE FOR METALLURGI-

CAL PURPOSES, ALSO BONE ASH, BORAX

GLASS, C. P. TEST LEAD

AND LITHARGE.

Manufacturers, Importers and Dealers in

ASSAYERS' AND CHEMISTS' SUPPLIES

C. \& P. AND HEAVY CHEMICALS

PHYSICAL AND BLOW-PIPE APPARATUS PROSPECTORS' OUTFITS.

The Largest Stock of

\section{SCIENTIFIC BOOKS}

in The West.

Wholesale and Retail Dealers in

PHOTOGRAPHIC SUPPLIES.

CATALOGUE MAILED ON APPLICATION.

When writing for same please state whether assay or photographic catalogue is desired. 


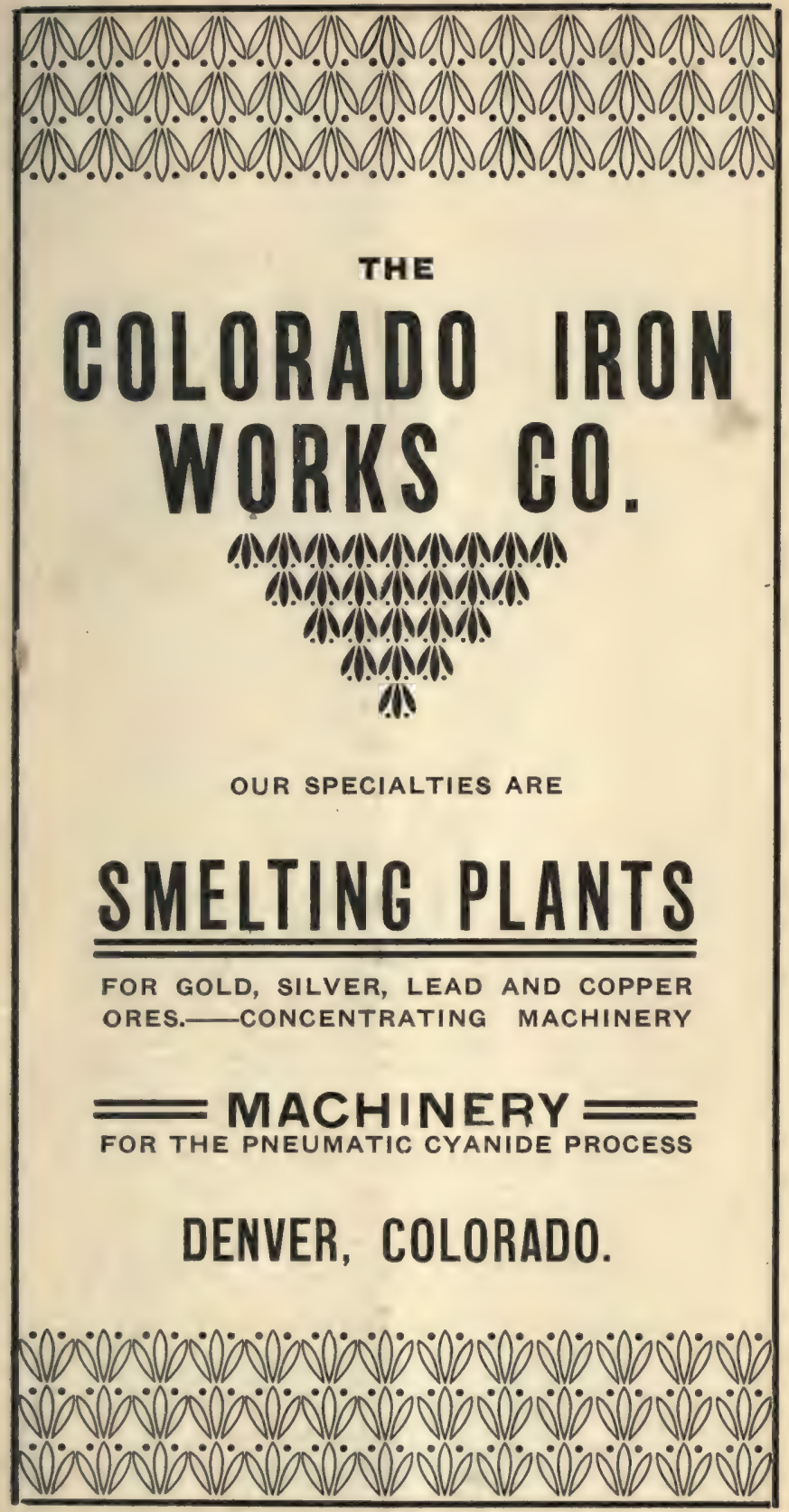




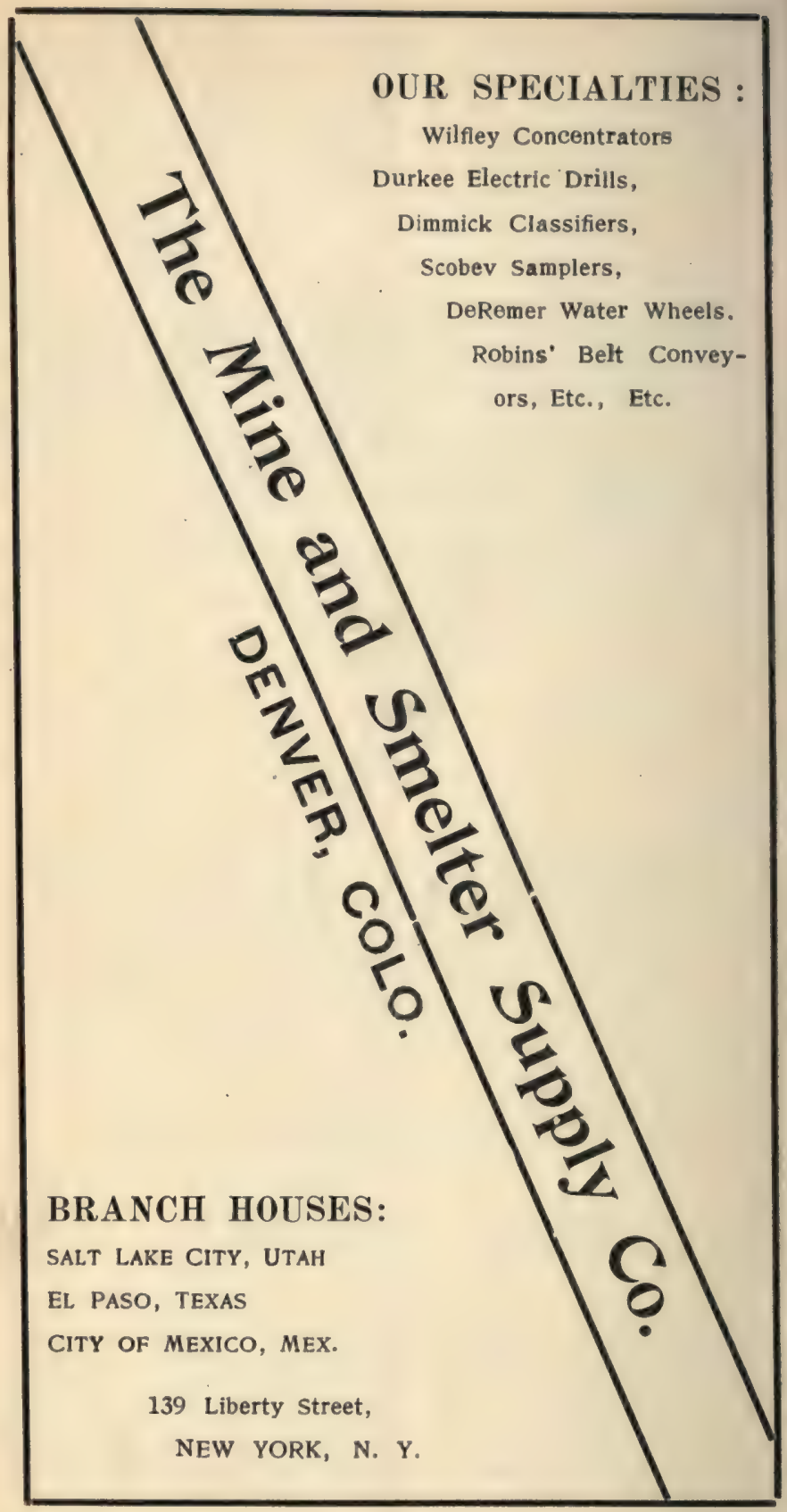





$$
5 p^{.321}
$$




\section{PLEASE DO NOT REMOVE CARDS OR SLIPS FROM THIS POCKET}

\section{UNIVERSITY OF TORONTO LIBRARY}

\section{S\&M A 321}


